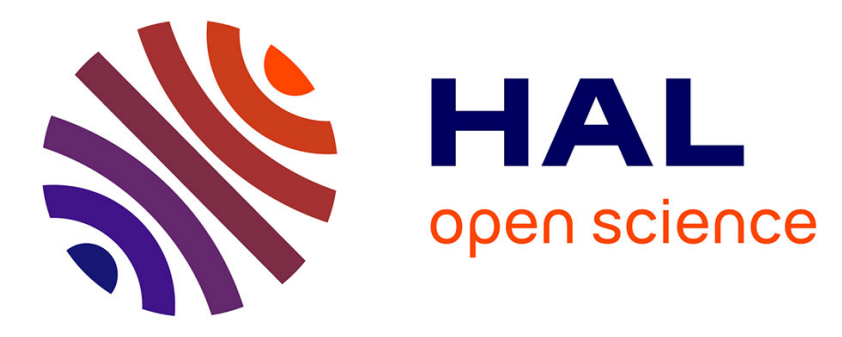

\title{
Bio-Sourced Polymers: Recent Advances
}

Henri Cramail, Boris Bizet, Oceane Lamarzelle, Pierre-Luc Durand, Geoffrey Hibert, Etienne Grau

\section{To cite this version:}

Henri Cramail, Boris Bizet, Oceane Lamarzelle, Pierre-Luc Durand, Geoffrey Hibert, et al.. BioSourced Polymers: Recent Advances. István T Horváth; Max Malacria. Advanced Green Chemistry Part 2: From Catalysis to Chemistry Frontiers, 6, World Scientific Publishing Company (WSPC), pp.167-328, 2020, Series on Chemistry, Energy and the Environment, 978-981-121-057-0. 10.1142/9789811210587_0005. hal-02886344

\section{HAL Id: hal-02886344 https://hal.science/hal-02886344}

Submitted on 1 Jul 2020

HAL is a multi-disciplinary open access archive for the deposit and dissemination of scientific research documents, whether they are published or not. The documents may come from teaching and research institutions in France or abroad, or from public or private research centers.
L'archive ouverte pluridisciplinaire HAL, est destinée au dépôt et à la diffusion de documents scientifiques de niveau recherche, publiés ou non, émanant des établissements d'enseignement et de recherche français ou étrangers, des laboratoires publics ou privés. 


\section{Bio-Sourced Polymers: recent advances}

Henri Cramail*, Boris Bizet, Océane Lamarzelle, Pierre-Luc Durand, Geoffrey Hibert and Etienne Grau

Laboratoire de Chimie des Polymères Organiques UMR5629

Université de Bordeaux - CNRS - Bordeaux INP/ENSCBP

16, Avenue Pey-Berland, F-33607 Pessac Cedex

email: cramail@enscbp.fr 
List of abbreviations

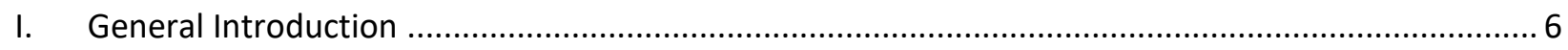

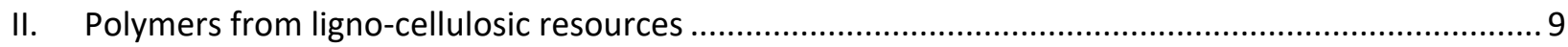

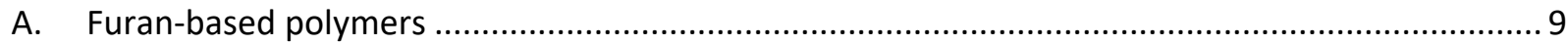

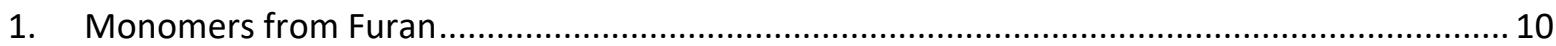

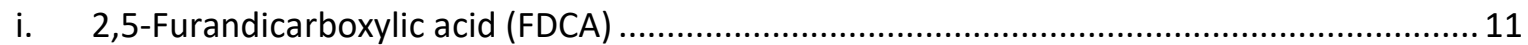

ii. Aldehyde- and aminomethyl-derivatives of HMF and FF ................................................. 11

iii. 2,5-Bis(hydroxymethyl)furan (BHMF) and Furfuryl alcohol (FF-OH) ................................... 12

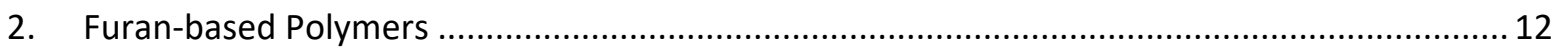

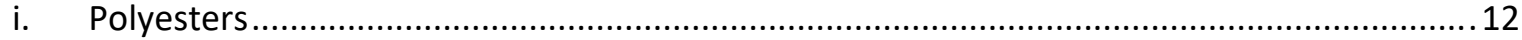

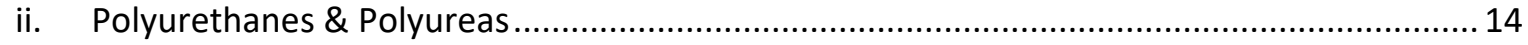

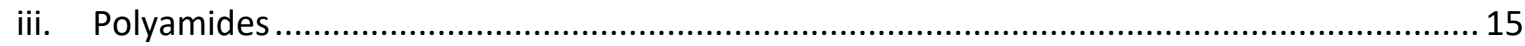

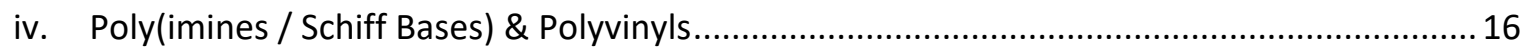

v. A reversible polymerization via a Diels-Alder process .......................................................... 17

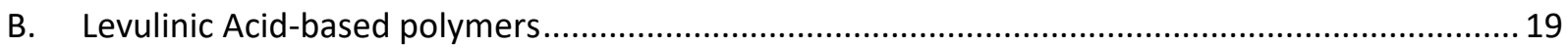

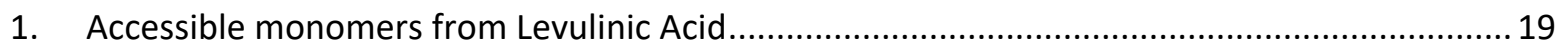

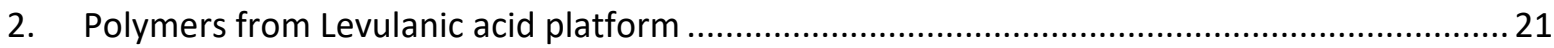

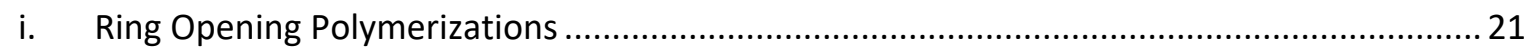

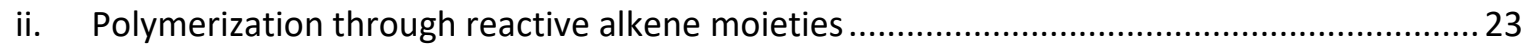

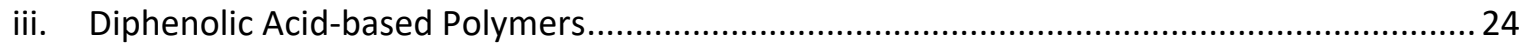

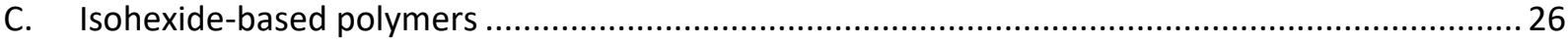

D. Synthons and/or polymers originating from biotechnological routes ........................................29

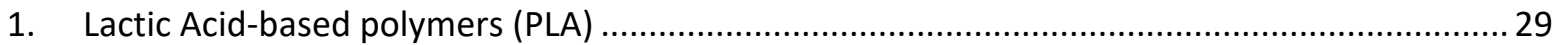

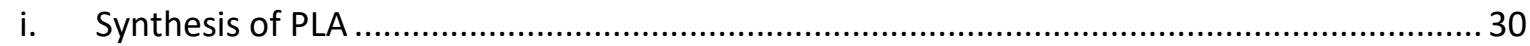

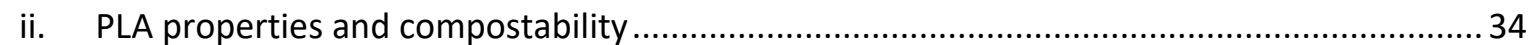

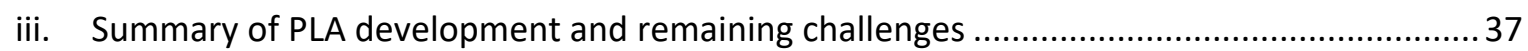

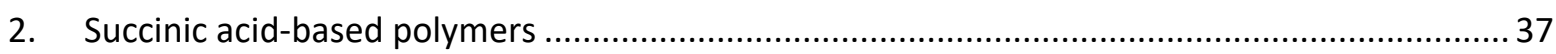

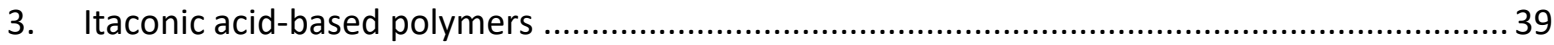

i. Chain-growth polymerization of Itaconic acid derivatives ................................................. 40

ii. Step-growth polymerization of Itaconic acid derivatives ...................................................... 41

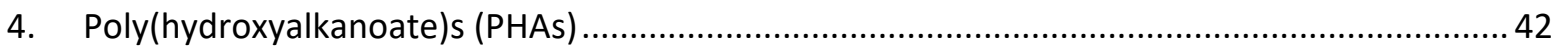

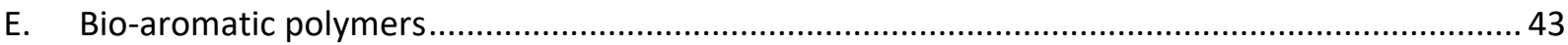

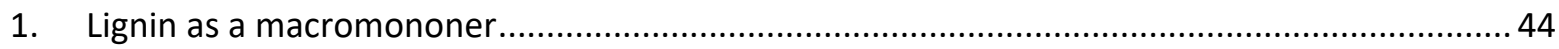

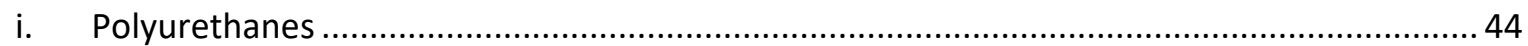




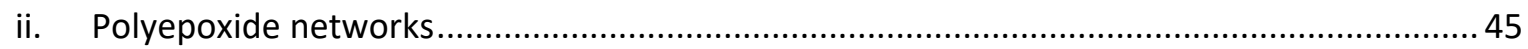

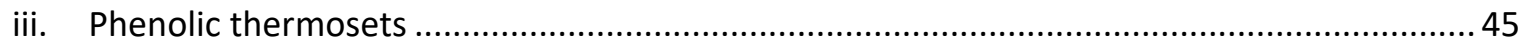

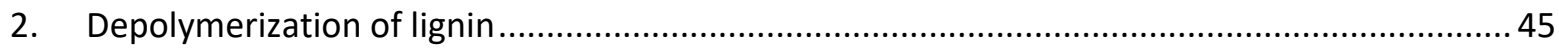

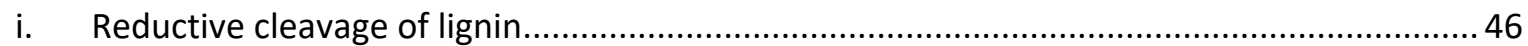

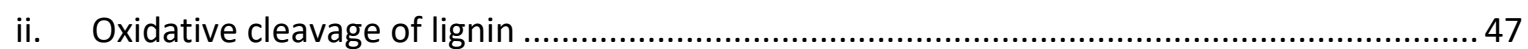

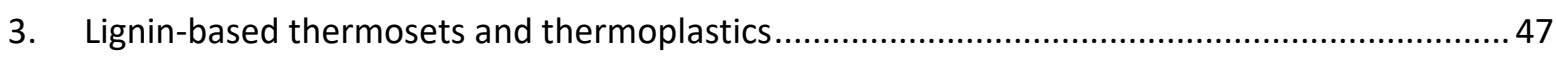

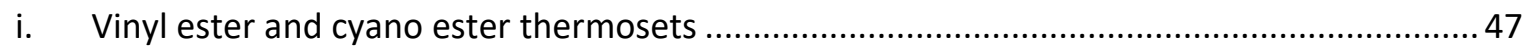

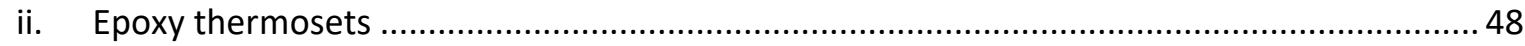

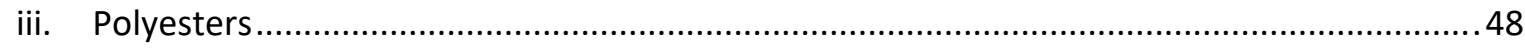

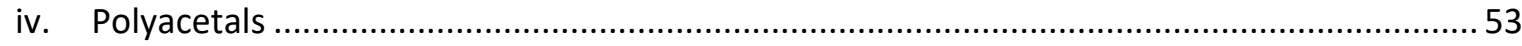

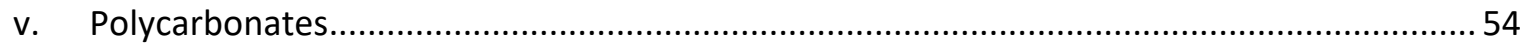

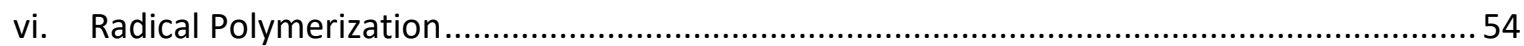

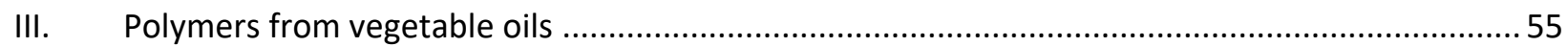

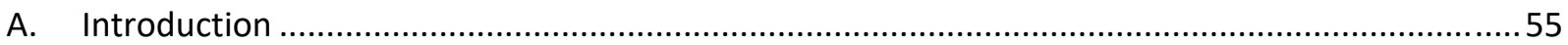

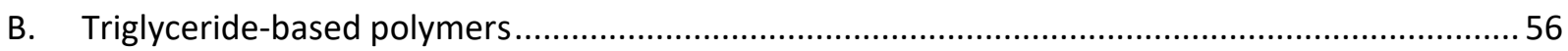

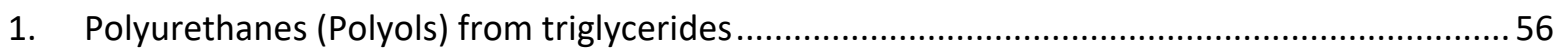

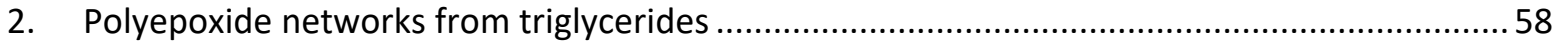

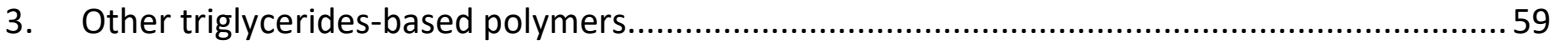

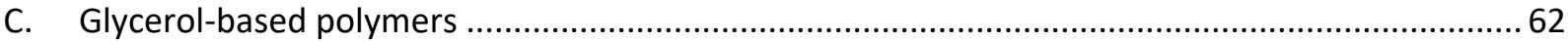

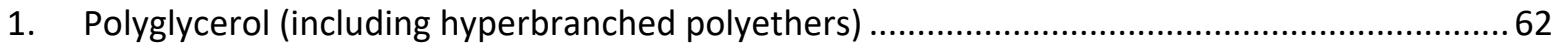

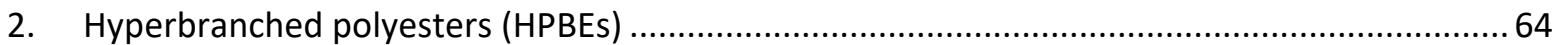

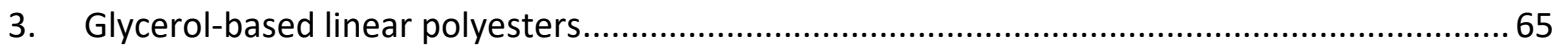

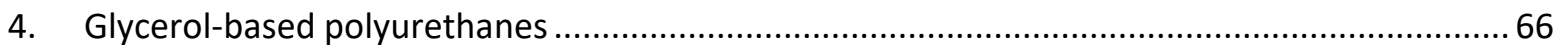

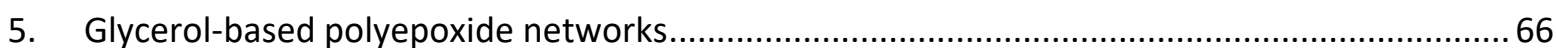

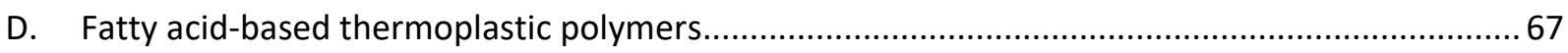

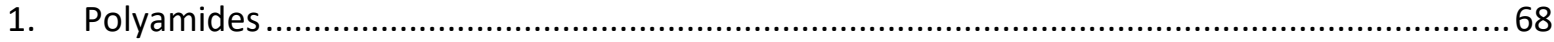

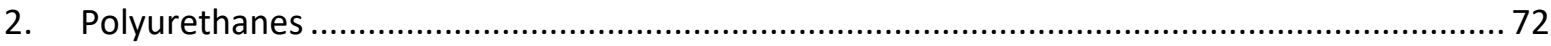

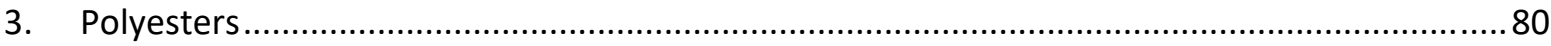

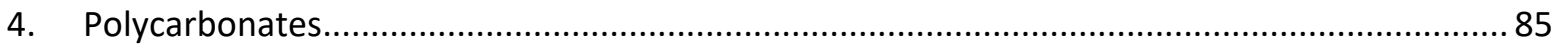

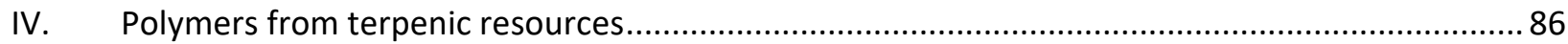

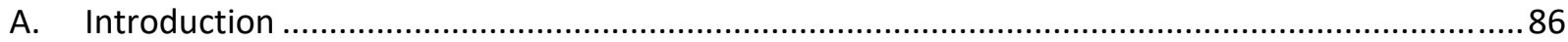

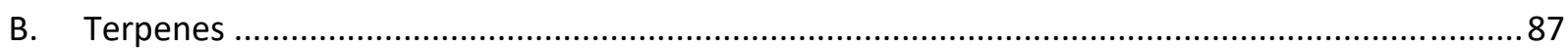

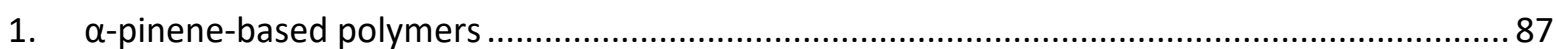

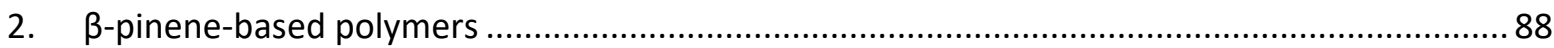




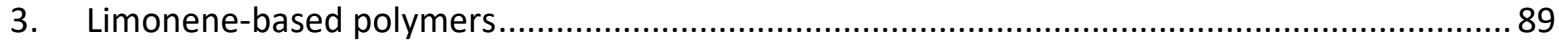

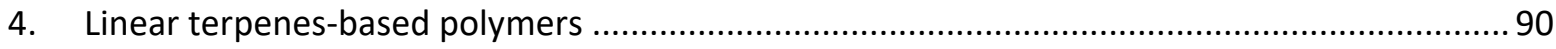

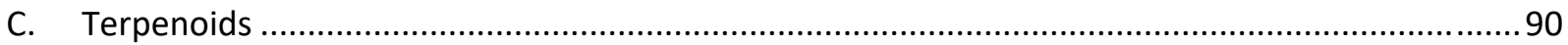

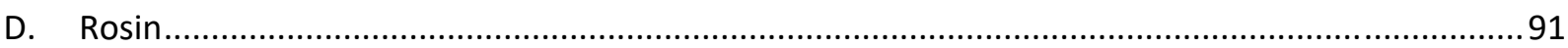

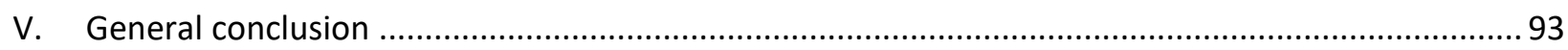

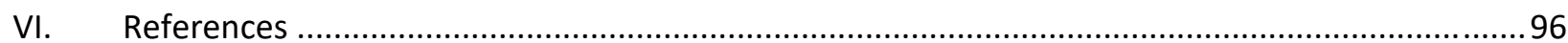

\section{List of Abbreviations}

ADMET: Acyclic diene metathesis

AFM: Atomic Force Microscopie

AIBN: Azobisisobutyronitrile ...

AL: Angelica lactone...

5-ALA: 5-aminolevulinic acid

Aq.: Aqueous

ATRP: Atom-transfer radical polymerization

BAMF: 2,5-bisaminomethylfurfural

BHMF: 2,5-bis(hydroxymethyl)furfural

bisCC: bis cyclic carbonates

BPA: Bisphenol-A

BTX: Benzene, toluene, xylene

CALB: Candida antartica lipase B

Cat.: Catalyst

CMF: 5-chloromethylfurfural

CRP: Controlled radical polymerization

CTA: Chain transfer agent

DA: Diels-Alder

DBTDL: Dibutyl tin dilaurate

DGEBA: Diglycidyl ether of bisphenol-A

DMA: Dynamic Mechanical Analysis

DMC: Dimethyl carbonate

DMF: Dimethylformamide

DMPA: 4-(Dimethylamino)pyridine

DPA: Diphenolic acid

DFF: 2,5-Diformylfuran

Đ: Polydispersity

EDA: Ethylene diamine

FAME: Fatty acid methyl ester

FDA: Food and Drug Administration

FDCA: 2,5-Furandicarboxylic Acid

FF: Furfural

GBL: $\gamma$-butyrolactone ...

GVL: $\gamma$-valerolactone ...

HDI: hexamethylene diisocyanate... 
HDPE: High density polyethylene

HDT: Heat distortion temperature

HMF: 2,5-Hydroxymethylfurfural

HPB: Hyperbranched polymer

HBPE: Hyperbranched polyester

IA: Itaconic Acid

IPDA: Isophorone diamine

IPDI: Isophorone diisocyanate...

LA: Levulinic Acid

LA: lactide or lactic acid

LAB: Lactic Acid bacterias

LCA: Life cycle analysis

LDPE: Low density polyethylene

MA: Methyl acrylate

mcl-PHA: Medium chain length poly(hydroxyalkanoate)

MEK: Methyl ethyl ketone

MGVL: $\alpha$-methylene- $\gamma$-valerolactone

MDI: 4,4'-Diisocyanate de diphénylméthane

MMA: Methyl methacrylate

$\mathbf{M}_{\mathbf{n}}$ : Number average molar mass

MTT: (3-(4,5-Dimethylthiazol-2-yl)-2,5-Diphenyltetrazolium Bromide)

$\mathbf{M}_{\mathrm{w}}$ : Mass average molar mass

NIPU: Non-isocyanate polyurethane

NMR: Nuclear Magnetic resonance

P3HB: Poly(3-hydroxybutyrate)

P3HHx: Poly(3-hydroxyhexanoate)

P3HO: Poly(3-hydroxyoctanoate)

P4HB: Poly(4-hydroxybutyrate)

PA: Polyamide

PAHB: Poly(alkylenehydroxybenzoate)

PBF: Poly(butylene furanoate)

PDLA: Poly(D-lactic acid)

PDLLA: Poly(D,L-lactic acid)

PBS: poly(butylene succinate)

PBT: poly(butylene terephtalate)

PCL: Poly( $\varepsilon$-caprolactone)

PE: Poly(ethylene)

PE: AUSSI UTILISE POUR LES POLYESTERS (p16)

PEA: Poly(ester amide)

PEF: Poly(ethylene furanoate)

PEG: Poly(ethylene glycol)

PEO: Poly(ethylene oxide)

PET: Poly(ethylene terephthalate)

Ph: Phenyl

PHA: Poly(hydroxyalkanoate)

PHU: Poly(hydroxy)urethane

PHV: poly( $\beta$-hydroxybutyrate)

PIC: Poly(ethylene carbonate) 
PIT: Poly(isosorbide terephthalate)

PLA: Poly(lactic acid)

PLimC: Poly(limonene carbonate)

PLLA: Poly(L-lactic acid)

PMMA: Poly(methylmetacrylate)

POM: Polyoxometalate

PP: Polypropylene

PRic: Poly(ricinoleic acid)

PS: Polystyrene

PTMO: Poly(tetramethylene oxide)

PU: Polyurethane

PVA: Poly(vinyl alcohol)

PVAc: Poly(vinyl acetate)

PVC: Poly(vinyl chloride)

RAFT: Reversible addition-fragmentation chain-transfer

rDA: retro Diels-Alder

ROcP: Ring-opening copolymerization

ROhP: Ring-opening homopolymerization

ROMP: Ring-opening metathesis polymerization

ROP: Ring-opening polymerization

RT: Room Temperature

SA: Succinic Acid

scl-PHA: Short chain length poly(hydroxyalkanoate)

SPAEK: Sulfonated poly(arylene ether ketone)

TBD: Triazabicyclodecene

TEMPO: (2,2,6,6-Tétraméthylpipéridin-1-yl)oxy

TDI: Toluene diisocyanate

$\mathbf{T}_{\mathbf{d x} \%}$ : Degradation temperature (when $\mathrm{x} \%$ of the product is degraded)

$\mathbf{T}_{\mathrm{g}}$ : glass transition temperature

THF: Tetrahydrofuran

$\mathbf{T}_{\mathrm{m}}$ : melting temperature

TPU: Thermoplastic polyurethane

$\mathbf{T}_{\beta}: \beta$-transition temperature

UPE: Unsaturated Polyester

USAXS: Ultra Small Angle X-ray Scattering

UV: Ultraviolet

wt\%: Weight percent

5CC: 5 -membered cyclic carbonate

6CC: 6-membered cyclic carbonate

\section{General Introduction}

Synthetic polymers are of major interest in the chemical industry, with a world production superior to $350 \mathrm{Mt}$ in 2016. They find applications in all the branches of industry, from packaging films to the stateof-the-art materials for sports and leisure activities, construction and aerospace industry or medical applications. Half of the amount of molecules produced by the petrochemical industry thus finds itself 
incorporated in the elaboration of polymer materials. With a production of $160 \mathrm{Mt} /$ year, ethylene can be considered as the major example of petroleum-based monomers. Green Chemistry introduced in 1998 by Anastase et al. (refer to Chapter 1 of this book), aims at providing solutions to reduce environmental impacts of our society. More precisely, this pursue of a more sustainable chemistry consists in using a source of renewable carbon to both reduce the dependence on fossil resources and thus stabilize greenhouse gas emissions (in particular $\mathrm{CO}_{2}$ ) at the end of life. The use of renewable resources is of major interest in the elaboration of bio-sourced polymers. By using them, it is possible to mimick the fossil-based polymers -drop-in structures such as biopolyethylene- or to design new chemical structures, such as poly(lactic acid), PLA. Nowadays, the vegetal-based chemistry (renewable resources) mobilizes less than $0.5 \%$ of arable land in the world.

Some reminders of the definitions of the terms biomass, biopolymer, biodegradable polymer, bio-sourced polymer, bioplastic, biorefinery may be necessary:

Biomass: material of biological origin (elaborated by alive bodies) with the exception of the materials of geological or fossil formation.

Biopolymer: polymer developed by alive bodies, extracted from the biomass. Examples: polysaccharides, proteins, bacterial polymers.

Biodegradable polymer: polymer the main degradation mechanism of which can be biotic, by enzymatic way. Under the action of micro-organisms and in the presence of oxygen (aerobic conditions), the organic compound decomposes totally within few months into carbon dioxide, water and mineral salts, with the appearance of a new biomass; in the absence of oxygen (anaerobic conditions), the organic compound decomposes totally within few months into carbon dioxide, methane, mineral salts and creation of a new biomass.

Bio-sourced (or bio-based) polymer: synthetic polymer partially (generally $>20 \%$ ) or totally obtained from by-products stemming from the biomass. The bio-sourced character of a polymer can be determined in particular from its content in C14, according to the standard ASTM D6866. For the materials of totally fossil origin, the content in $\mathrm{C} 14$ is null.

Bioplastic: term of popularization which indicates a 'biobased plastic' (restricted definition) and/or biodegradable (wider definition).

Biorefinery: by analogy with a classic refinery, which works from fossil resources, a bio-refinery handles biomasses to produce, according to the cases, energy, fuels, materials, chemical and polymer products and/or animal and human feed.

Today, two biopolymers account for a significant part of the world polymer consumption: the 1,4-cis polyisoprene (natural rubber, approximately 12Mt/year) - precursor of elastomers - and the cellulose stemming from wood pulps (regenerated cellulose, ethers and esters of cellulose, for a total about $5 \mathrm{Mt} / \mathrm{year}$ ). As for bio-sourced polymers, the latter reaches approximately $1 \%$ of the global production of polymer materials, but their production knows a considerable development and corresponds to the category of polymers the dynamics of which is the most important, both in terms of production and innovation (Figure 1). 


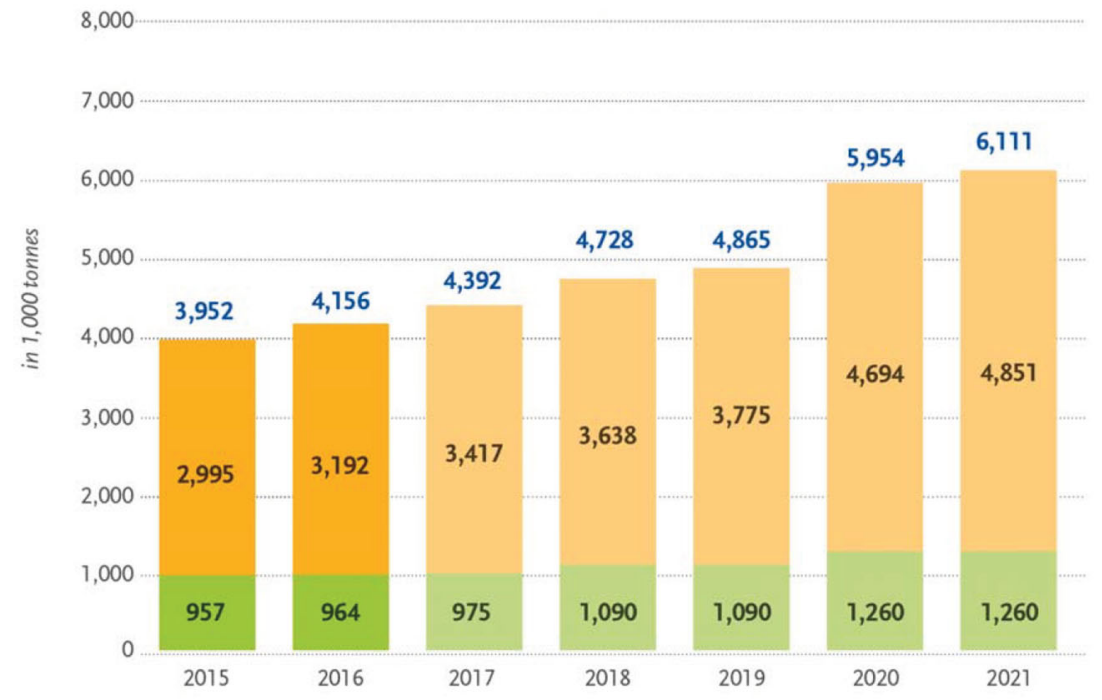

Figure 1. Trend and Capacity of production of 'Bioplastics' in Ktons (from 2015 to 2021). 2 distinct classes: the non-biodegradable bio-sourced polymers such as bio-polyethylene, BioPE, bio-poly(ethylene terephtalate), BioPET, various bio-based polyamides, PA ...(in orange), and the biodegradable bio-based polymers such as the ones based on starch, the polylactide, PLA, ... (in green). Source: European Bioplastics, Nova-Institute (2016)

The current development of biosourced polymers is first motivated by the search for performances and for new features. Indeed, in a global approach, which has to take into account the price of these new renewable resources, the bio-sourced feature is not enough for the industrial development of these new polymers. Besides, the reduction of impacts, in particular via the use of less toxic monomers and catalysts, is a driving force to the development of the renewable resources. The increase of the legislative arsenal - with in particular the regulation REACH - aims indeed at reducing the exposure to dangerous substances of the industry and end-users employees. In the polymers' field, Green Chemistry can be an answer to these challenges, by proposing less dangerous and possibly biosourced monomers (and catalysts). In every case, the danger of a substance does not come from its composition in renewable carbon but from its chemical structure and the qualifier " biosourced " or "natural" does not mean without any danger! Identical substances will bring the same properties and the same dangers. The only advantage - in the short term - is to possess industrial facilities of transformation and existing application sectors. The regulations however consider all the new molecules - may they be biosourced - on an equal basis, and the industrialists have to study their effects on the Man and the Environment before their launch on the market, which is very expensive and limits the development of new products.

Today, the development of bio-sourced polymers is in particular based on the valorization of two main types of resources at the industrial level: oleaginous plants and polysaccharides, in connection with the corresponding agricultural productions. ${ }^{1}$ Indeed, numerous development projects and some already marketed products concern the valuation of vegetable oil and glycerin for the elaboration of precursors of polymers such as polyamides, polyurethanes, polyesters and polyethers with world actors as Arkema, Oleon (Avril), Evonik, Elevance, HunsTman in Europe and US Biobased in the USA. Also, the starchy and saccharide-based resources are under industrial development for the elaboration of polyols, polyesters, solvents, etc. with group world-leaders as Roquette, Tereos, Solvay, NatureWork. The United States developed very early (before 2004) a strategy targeted with a strategic vision of the DoE (DeparTment of Energy) for the industrial development of 12 synthons (building blocks), strategic for the American economy (see Figure 2). 
Brazil made the industrial choice around the valuation of resources targeted as the sugar cane with, in particular, the production of biosourced polyethylene (Braskem company) by polymerization of ethylene stemming from the dehydration of the ethanol obtained by fermentation. It is in particular this bioethylene which is used to develop bio-poly(ethylene terephtalate), bioPET. Other actors are interested in the valuation of extractable compounds stemming from biomass, such as terpenes for applications as adhesives notably. Finally, numerous research efforts are dedicated today to the valuation of lignin stemming from various plentiful ligno-cellulosic resources - in particular by deconstruction of this one to propose aromatic synthons of interest.<smiles>O=C(O)CCC(=O)O</smiles>

Succinic acid<smiles>NC(CC(=O)O)C(=O)O</smiles>

Aspartic acid<smiles>C=C(CC(=O)O)C(=O)O</smiles>

Itaconic acid<smiles>OCC(O)CO</smiles>

Glycerol<smiles>O=C(O)c1ccc(C(=O)O)o1</smiles>

2,5-Furane dicarboxylic acid<smiles>O=C(O)[C@@H](O)[C@@H](O)[C@H](O)[C@@H](O)C(=O)O</smiles>

Glutaric acid<smiles>CC(=O)CCC(=O)O</smiles>

Levullinic acid<smiles>OC[C@@H](O)[C@@H](O)[C@H](O)[C@H](O)CO</smiles>

Sorbitol<smiles>O=C(O)CCO</smiles>

3-hydroxy Propionic acid<smiles>N[C@@H](CCC(=O)O)C(=O)O</smiles>

Glutamic acid<smiles>O=C1CC(O)CO1</smiles>

3-hydroxy Butyrolactone<smiles>OC[C@@H](O)C(O)[C@H](O)CO</smiles>

Xylitol

Figure 2. List of the twelve strategic 'building blocks' targeted by the US DeparTment of Energy (DoE)

\section{II.Polymers from ligno-cellulosic resources}

\section{A. Furan-based polymers}

The use of furans in polymer chemistry has attracted a tremendous attention in the last decades. This growing interest can be attributed to several parameters, such as the relatively easy access to sugar sources as well as political incentives depending on each country. Directly accessible from carbohydrate resources, furan derivatives are widely available and can thus be considered as serious candidates for the synthesis of commodity and/or advanced value-added products. However, the apparent easy access to sugar sources must not prevent the fact care has to be devoted not to enter in competition with the food industry. As such, the selective depolymerization of sugar-originating biopolymers such as cellulose, hemicelluloses, high-fructose corn syrups (among others) has become a potential solution to yield interesting platform molecules. 
Due to the extremely high amount of available literature in the field, it seems almost impossible to perform a thorough review of all the results. It is however worth mentioning the work of Gandini and Naceur Belgacem ${ }^{2}$ who extensively reviewed a wide scope of monomers and polymers which can originate from furan-derivatives. It is strongly encouraged to refer to this work, should complementary information be needed.

\section{Monomers from Furan}

As described before, furan-based monomers can be accessed through the depolymerization of sugaroriginating biopolymers. 5-Hydroxymethylfurfural (HMF) and furfural (FF) are the main platform molecules that can be synthesized from sugar-sources. Acidic dehydration of cellulose yields HMF whereas hemicelluloses yield FF (Figure 3) ${ }^{3-5}$ From them, an almost unlimited number of interesting monomers can be accessed, depending on the employed synthetic pathway.

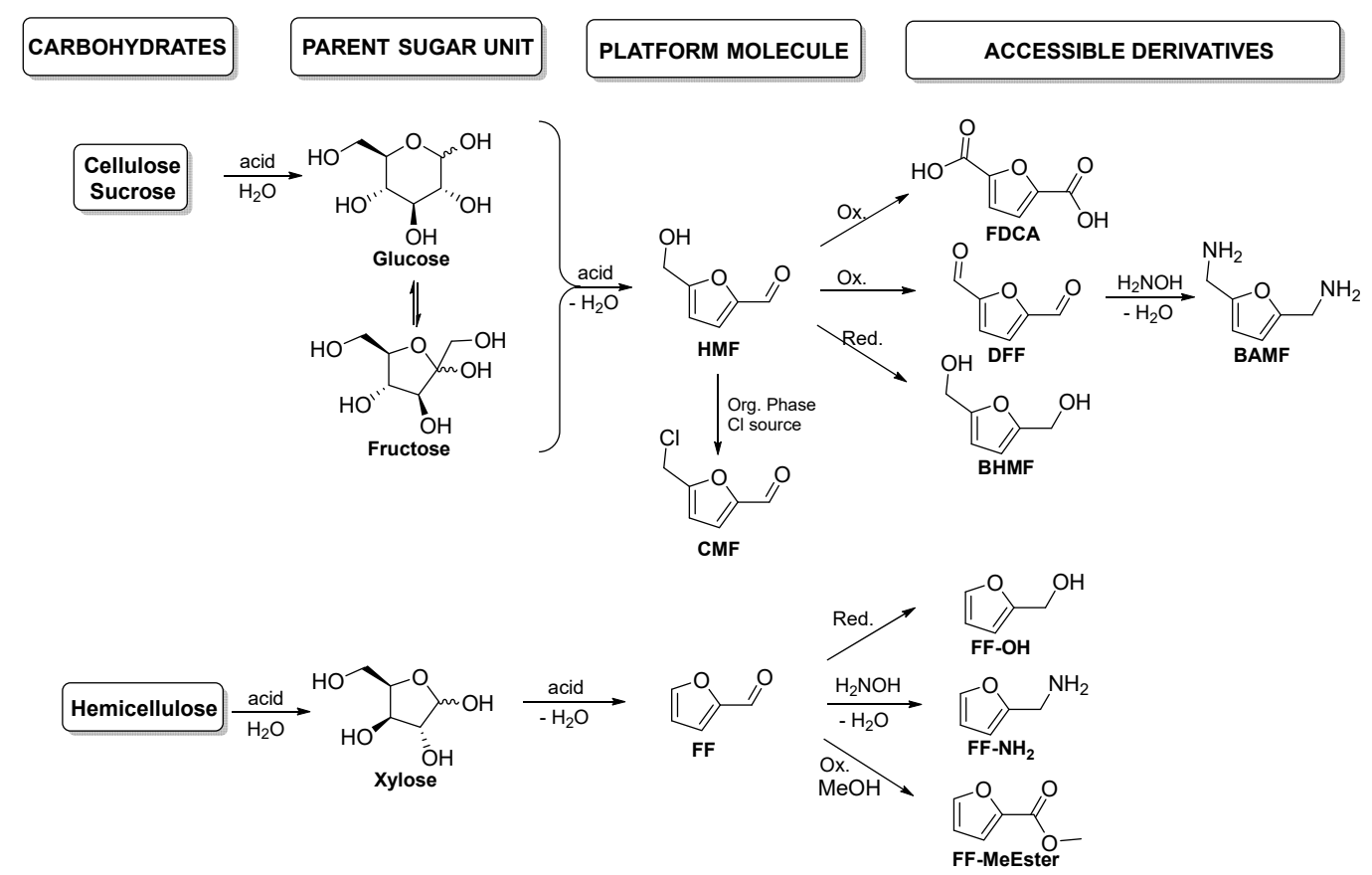

Figure 3. Possible derivatives from the furfural platform

Describing the different pathways from cellulosic resources to FF and HMF is out of the scope of this chapter, but extensive information can be found in the previous cited reviews. However, an important aspect of the production of HMF, still slowing down its industrial implementation, has to be mentioned. The acidic dehydration process in aqueous phase usually requires harsh to very harsh conditions (such as high temperatures and high-to-very-high acidic concentrations). While reacting, cellulose and hemicelluloses are breaking up to yield glucose, fructose and xylose moieties, which can rearrange in acidic conditions to produce so-called humins (or humic matter), a high-carbon-content brown material, that has - to our knowledge - no existing valorization process (except burning). In the case of HMF, it was found that this reaction was favored within the aqueous phase, which is not desirable. The use of biphasic reaction condition with the addition of an in situ extracting immiscible organic solvent during the reaction exhibited superior results in terms of decreased amounts of humins. Recent discoveries ${ }^{6,7}$ showed that using a chlorine source $(\mathrm{HCl})$ favored the formation of 5-chloromethylfurfural (CMF) as opposed to HMF. Let alone leaving a very reactive methyl chlorine moiety in place of the hydroxyl group, this 
molecule possesses the advantage of having a very poor solubility in water so that, when using an aqueous-organic biphasic system, in situ extraction could occur right after formation of CMF in the aqueous phase. The molecule is thus protected from the very acidic aqueous phase and less humic matter is formed. Depending on both the sugar source and the utilized process (batch vs flow chemistry), high yields could be reached, leaving a lot of room for improvement towards process economics and further chemical reactivity. However, very few examples of CMF conversion ${ }^{8-10}$ are encountered in the literature. This is why only HMF derivatives will be addressed in the following sections.

\section{i. 2,5-Furandicarboxylic acid (FDCA)}

The formation of 2,5-Furandicarboxylic acid (FDCA) relies on the oxidation of HMF to yield its diacid derivative. ${ }^{5}$ The rate, as well as the selectivity of the reaction, obviously is catalyst-dependant and the number of possible processes is very high. The homogeneous catalytic oxidation of HMF with $\mathrm{O}_{2}$ is performed thanks to the Amoco Mid-Century process, in which a mixture of $\mathrm{Co} / \mathrm{Mn} / \mathrm{Br}$ salts is utilized in acetic acid. Depending on both the use of co-catalysts and the process conditions, up to $90 \%$ yield of FDCA could be obtained, even if the majority of the obtained yields were reported to be around $60 \%$. However, the acidic conditions are a limitation to this reaction since it favors the degradation of the native HMF (to humins for instance). This is a reason why other pathways, involving noble metals in addition to an external base, were investigated. As far as noble metals are concerned, $\mathrm{Pt}, \mathrm{Au}, \mathrm{Pd}$ are the most studied catalysts. They all gave the best yields when utilized with an external base in water, which is an improvement as it prevents from the degradation of $\mathrm{HMF}(>90 \%$ yield). Supported, meaning heterogeneous, catalysis was also proven to be suitable for such a process, which is an improvement in terms of recyclability. As a matter of comparison, supported-Au was demonstrated to give better yields as opposed to Pt and Pd-supported catalysts. Another advantage of such a process is its versatility, because a lot of freedom is left to the operators with the choice of the base or the combination of catalysts. However, it is to be noted that the obtained FDCA is never pure, but its $\left(\mathrm{Na}_{2} / \mathrm{K}_{2} / \mathrm{Mg}\right.$-)salt version (depending on the utilized base). This salt formation is the limiting step towards industrialization since it requires complicated purification steps to form and isolate pure FDCA from water. A potential solution is to perform this reaction in Methanol, yielding the methyl-ester form of FDCA. Interestingly, this protocole can also be transferred to furfural (FF) in the production of the corresponding methylester (FFMeEster, see Figure 3). Finally, little amount of literature described the use of supported base metals for the production of FDCA. Fe and Co-based catalysts were mostly investigated. Yields up to $90 \%$ could be obtained depending on the process conditions and the used oxidant. Other alternatives, such as the use of ionic liquids have also been reported. ${ }^{11}$

\section{ii. Aldehyde- and aminomethyl-derivatives of HMF and FF}

The selective oxidation of HMF to yield 2,5-Diformylfuran (DFF, Figure 3) has also been reported. One of the advantages of making DFF as opposed to FDCA is the higher solubility of DFF in organic solvents. As such, some examples of forming DFF directly from cellulosic resources 1 pot-1 step process can be encountered. In a similar fashion with CMF, this proceeds through the aqueous formation of HMF followed by the in situ extraction into the organic solvent, in which the oxidation step can be performed. In order to oxidize HMF to DFF, specific metal catalysts have been investigated. Ru-supported catalysts gave good selectivity towards DFF and yield both higher than $95 \%$. Vanadium-based catalysts were also utilized even if they demonstrated lower yields $(<85 \%)$. Finally, $\mathrm{Mn}-$ and $\mathrm{Cu}$ - based catalysts, in combination with TEMPO as the oxidizing component, have also been highlighted as potential solutions in the oxidation of HMF to DFF. 
Starting from DFF, it is then possible to form the diamine form of HMF: 2,5-Bis(aminomethyl)furan (BAMF, Figure 3). A possible process, described in a patent ${ }^{12}$ involves a 2-step process: a first step in which DFF reacts with hydroxylamine $\mathrm{H}_{2} \mathrm{NOH} . \mathrm{HCl}$ to form the bis-oxime derivative of $\mathrm{HMF}$, followed by a reduction step via $\mathrm{Ni}$ catalyzed reaction of $\mathrm{H}_{2}$ onto the bis-oxime to yield BAMF. Other process involving supported $\mathrm{Ni}, \mathrm{Cu}$ and $\mathrm{Ru}$ catalyst have been described for the production of BAMF from DFF. In a first step, DFF is reacted with ammonia in a gas phase to from the corresponding bis-imine compound. This compound is then hydrogenated with the help of the aforementioned catalysts to yield BAMF with high conversions and selectivities. ${ }^{13}$

\section{iii. 2,5-Bis(hydroxymethyl)furan (BHMF) and Furfuryl alcohol (FF-OH)}

In a similar fashion to oxidation process, the reduction of HMF and FF can yield a large number of products depending on the reaction conditions. As such, if a selective reduction of the aldehyde moiety can yield BHMF and FF-OH from HMF and FF respectively, it is worth keeping in mind that the reduction of the furan ring and its successive ring-opening are still doable if too harsh reaction conditions are encountered.

Ru-based catalysts exhibited a good selectivity towards BHMF. ${ }^{14}$ However, it is to be noted that a lot of other metal catalysts can be suitable for the reaction when adapted reaction conditions are employed. Sn-, $\mathrm{Cu}$-, Pt- Pd- and Au-based catalysts were proven to be efficient. ${ }^{15-19}$ Interestingly, an Ir-based catalyst was highlighted as a promising candidate for the hydrogenation reaction of HMF and FF into BHMF and FF$\mathrm{OH}^{20}$

Finally, the Cannizzaro reaction was also proven to be a potential route towards the alcohol-derivatives of both $\mathrm{HMF}$ and $\mathrm{FF}$. It consists in making a base, such as $\mathrm{NaOH}$, react the aldehyde moiety of the furfural compounds. A good selectivity in water at $0^{\circ} \mathrm{C}$, with a maximum yield of $86 \%$, was reported. ${ }^{21}$

\section{Furan-based Polymers}

\section{i. Polyesters}

Among the accessible classes of furan-based polymers originating from step-growth polycondensation, polyesters certainly are the most studied. When analyzing the different derivatives that can be obtained from the 5-HMF platform, the presence of diols and diacids justifies why a lot of studies have been performed on the synthesis of various polyesters.

FDCA has particularly attracted a lot of attention as a bio-based alternative to terephtalic acid. This aromatic diacid, classified by the DoE as one of the 12 top value-added chemicals originating from biomass (see Figure 2), has been earmarked for its ability as substitute of terephtalic acid. Since 1951 and the publication of the first patent claiming the preparation of a polyester from FDCA and ethylene glycol, ${ }^{22}$ several pathways have been investigated towards the synthesis of polyesters by reacting FDCA (or its dimethyl-ester derivative) with diols -glycols in particular- (Figure 4). While the first pathway consists in a direct polycondensation, the second is based on a transesterification process. The choice of the diol has obviously an influence on the resulting properties of the polyesters so-formed, in terms of dimensions and thermomechanical properties. ${ }^{23-33}$ 


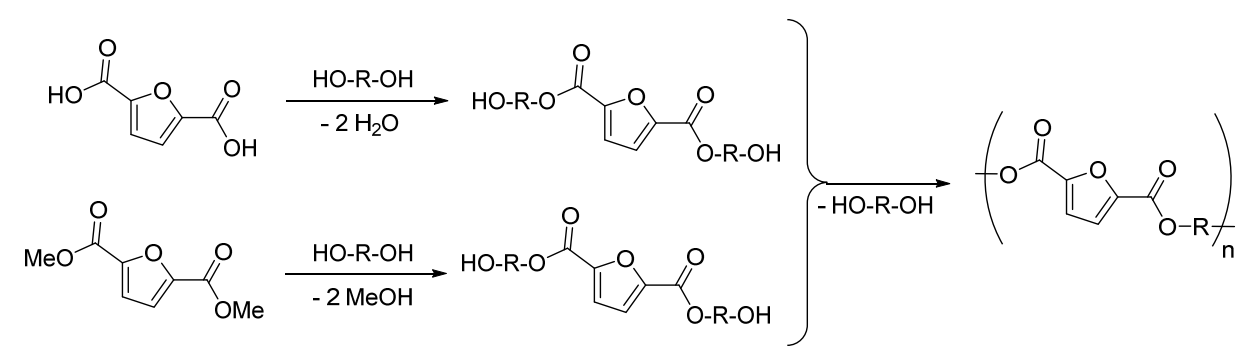

Figure 4. Synthesis of polyesters from 2,5-furan dicarboxylic acid (FDCA)

It is worth noting that transesterification reaction is usually performed in the presence of a catalyst following a procedure close to the one of PET synthesis. Lewis catalysts (such as $\mathrm{Sb}_{2} \mathrm{O}_{3}, \mathrm{Ca}(\mathrm{OAc})_{2}$, $\mathrm{Zn}(\mathrm{OAc})_{2}$ ot Titanium(IV) alkoxides) have proven their efficiency. ${ }^{5}$

Poly(ethylenefuranoate) (PEF) and poly(butylenefuranoate) (PBF) are particular targets of interests. Both are indeed $100 \%$ bio-based polyesters that have already been implemented at an industrial scale (Aventium, Coca-Cola). ${ }^{34,35}$ Burgess at al. demonstrated that PEF exhibited superior properties to its petrochemical analogue (PET) notably in terms of gas permeability. ${ }^{36-38}$

It is interesting to mention that from a historical point of view, industrials showed a huge interest in HMF and FDCA for their ability to be converted into bio-sourced terephthalic acid for the production of biosourced PET. The corresponding processes have been extensively described in a review co-authored by five different companies. ${ }^{39}$ Two main pathways emerge from their description, both of them relying on a reaction of HMF or FDCA with ethylene through a Diels-Alder reaction (Figure 5).

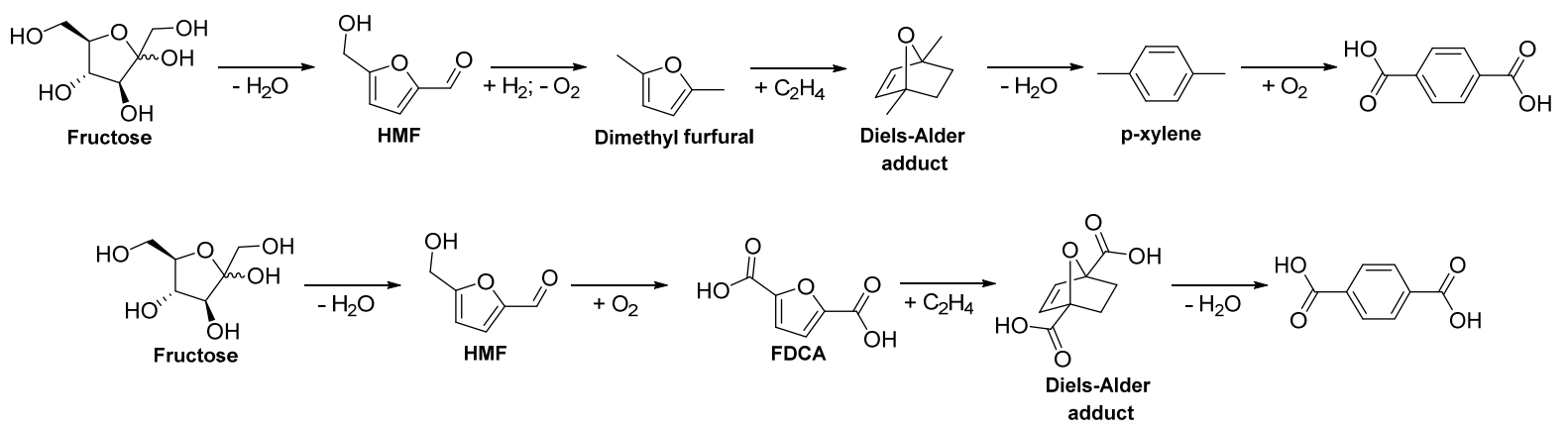

Figure 5. Synthesis of bio-based terephthalic acid from 5-hydroxymethyl furfural and 2,5-furan dicarboxylic acid

The first pathway relies on the reduction of HFM to Dimethyl furfural and has been the first to be studied. Numerous research teams optimized the process, which led Lin et al. to study, in 2013, its technoeconomic aspects. ${ }^{40}$ The authors concluded that the production cost would be about 2.5 times the recent price of petroleum-derived p-xylene (in 2014), with around $80 \%$ of the cost originating from the needed raw materials such as HMF, hydrogen and ethylene. The second pathway relies on the use of FDCA. As a matter of comparison, this process takes advantage of the high oxygen amount contained in FDCA to yield terephthalic acid. This allows avoiding the reduction step observed in the first pathway and thus decreases the number of steps required to form the final product. Both processes have a theoretical weight yield of $61 \%$. To our knowledge, it is today unfortunately impossible to compare the techno-economic aspects of this process as opposed to the first pathway.

Achieving the formation of polyesters can also be possible when starting with the diol-derivative of 5HMF. 2,5-bis(hydroxymethyl)furan (BHMF) can thus be reacted with diacids to yield the corresponding polyester (Figure 6). 


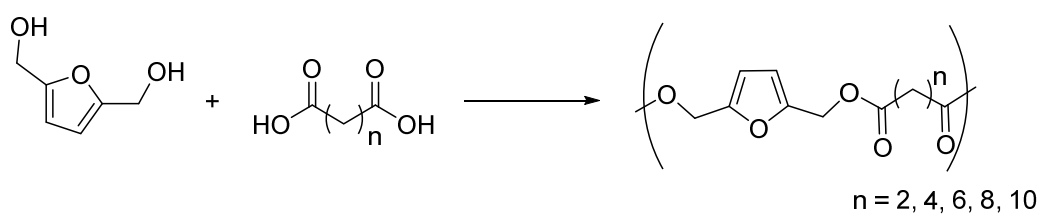

Figure 6. Synthesis of polyesters from 2,5-bis(hydroxymethyl)furan (BHMF)

Yoshie et al. thus demonstrated in a series of papers the possibility to perform the polycondensation reaction of BHMF with succinic acid $(n=2)$ under basic conditions. ${ }^{41-44}$ Very interestingly, self-healing capability was observed with the resulting polyester upon addition of bismaleimide cross-linker; more details will be provided in paragraph 2.2.5.

Using lipases through an enzymatic catalytic process, Loos et al. also described the esterification process of BHMF with a series of diacids of various chain lengths as described in Figure $6 .{ }^{45}$ Relatively low molecular weights ranging from 1600 to $2400 \mathrm{~g} \cdot \mathrm{mol}^{-1}$ were obtained.

Finally, it is worth mentioning the possibility to form bio-sourced furan-derived polyesters originating from dimer forms of furan-carboxylic acids. This 2-step process first requires an oxidation of Furfural (FF). As described previously, extensive work has been undertaken in order to find an industrially attractive way of oxidizing HMF to 2,5-FDCA, the most promising results of which being in methanol over gold catalyst. ${ }^{46,47} \mathrm{FF}$ can be also oxidized in a very similar fashion. ${ }^{48-51}$ The obtained product can thus be dimerized with the help of a carbonyl compound (aldehyde or ketone) through a condensation step in concentrated $\mathrm{H}_{2} \mathrm{SO}_{4}$ at $0^{\circ} \mathrm{C}$, (see Figure 7).

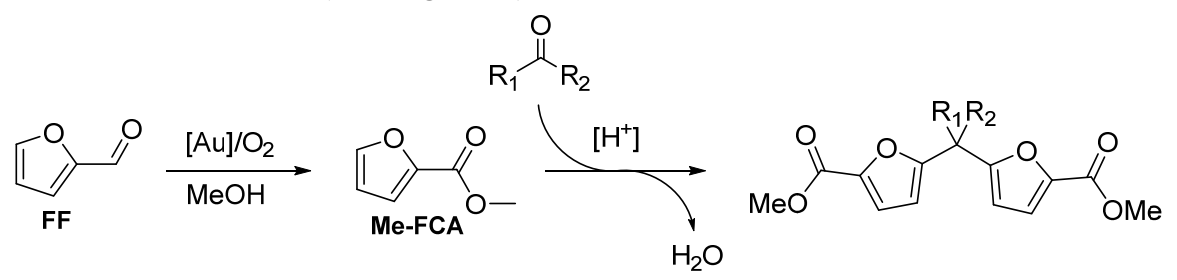

Figure 7. Synthesis of diacid (diester) compounds originating from a dimerization process of furfural

Good yields of $80-84 \%$ were obtained when formaldehyde $\left(\mathrm{R}_{1}, \mathrm{R}_{2}=\mathrm{H}\right)$ and acetaldehyde $\left(\mathrm{R}_{1}=\mathrm{CH}_{3}\right.$, $\mathrm{R}_{2}=\mathrm{H}$ ) were used. Stronger reaction conditions, namely a temperature of $60^{\circ} \mathrm{C}$, were necessary to achieve the formation of a dimer with acetone $\left(\mathrm{R}_{1}=\mathrm{CH}_{3}, \mathrm{R}_{2}=\mathrm{CH}_{3}\right)$ and a moderate yield of $65 \%$ was obtained. ${ }^{52,53}$ Such dimers are particularly interesting as they can be used as bio-sourced bis-aromatic monomers, providing specific mechanical properties to the final polyesters (Figure 8).

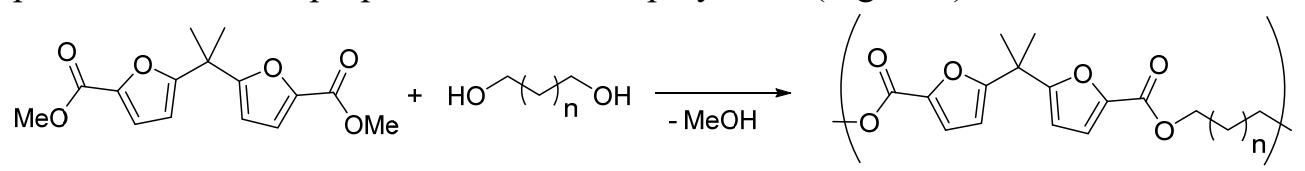

Figure 8. Synthesis of polyesters from furfural-dimer-based diesters

Khrouf et al. used the potentially bio-sourced ethylene glycol, 1,3-propanediol and 1,4-butanediol as comonomers in the transesterification reaction with the furan dimers. ${ }^{54}$ Melt polymerization can be employed if desired due to the very high stability of the monomers. It is also interesting to mention that the diacyl chloride form of the dimers can also be advantageously utilized. ${ }^{55}$ Finally, such dimers have more recently been employed in the synthesis of copolymers such as block copoly(esters) with PET. ${ }^{56}$

\section{ii. Polyurethanes \& Polyureas}


Due to a very broad scope of applications, the polyurethane industry nowadays has a great interest in substituting current petroleum-based polymers with bio-sourced products in order to address a growing sustainability issue. Traditionally, polyurethanes originate from the polycondensation reaction between a diol and a diisocyanate. Even if the use of diisocyanates (needing toxic phosgene) can be questionable from a bio-sourced mindset, it is worth mentioning that a profitable process today relies on the use of diisocyanate at an industrial scale. This is why isocyanate-dependant routes will be presented in this section. Given the fact that polyureas can be formed in a very similar fashion as opposed to polyurethanes, their formation will also be discussed thereafter.

When considering the panel of platform molecules accessible through the dehydration process of cellulosic resources, it is easy to notice that 2,5-bis(hydroxymethyl)furan (BHMF) can be used straightly by means of reaction with diisocyanate to form the corresponding polyurethane products. (Figure 9)

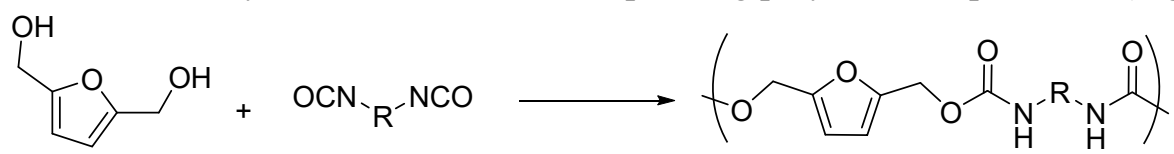

Figure 9. Synthesis of polyurethanes from 2,5-bis(hydroxymethyl)furan (BHMF)

The Quaker Oats Co. thus patented in 1982 and 1984 the use of BHMF in order to form polyurethane foams. BHMF, methylated-BHMF, BHMF homopolymer or BHFM-Propylene oxide copolymer could be reacted with a wide scope of polyisocyanate compounds (Papi® supplied by Dow ${ }^{\circledR}$ ) to yield foams exhibiting interesting flame-retardency ability. ${ }^{57-59}$

Finally, and following the similar pathways than those observed for polyesters, FF can be dimerized and transformed to get a diisocyanate, that can further be reacted with polyols to yield polyurethanes, (Figure 10).

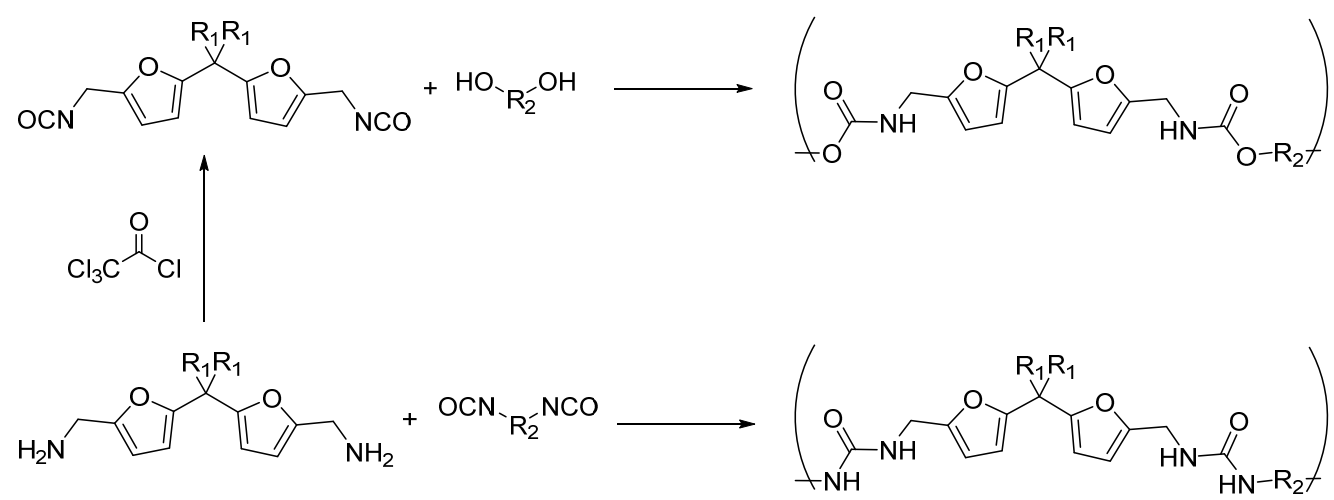

Figure 10. Synthesis of polyurethanes and polyureas from furfurylamine dimers and derivatives

By converting the furfuryl amine dimer into their diisocyanate counterparts, Cawse et al. synthesized polyurethane materials. The resulting polymers exhibited similar properties when compared to the bis(4isocyanatophenyl)methane (MDI)-based petroleum analogues. The authors thus displayed a bio-sourced alternative for the synthesis of industrially relevant polymeric materials. ${ }^{60-62}$ Attempts were also performed by the team of Gandini for the synthesis of $100 \%$-furanic compounds. ${ }^{63,64}$ The isocyanatederivated dimer of FF was thus reacted with various furan-based diols for making final polymers with tunable properties.

It is finally worth mentioning that furfurylamine dimer can also be converted into various polymers. Its reaction with diisocyanates yields for instance polyureas, but many other kinds of polymeric materials can be designed thanks to the versatility of the monomer.

\section{iii. Polyamides}


Regarding the synthesis of bio-sourced polyamides from furanic resources (Figure 11), quite scare examples have been highlighted in the scientific literature. Bis(aminomethyl)furan (BAMF) thus emerges as one of the most logical compound to utilize.

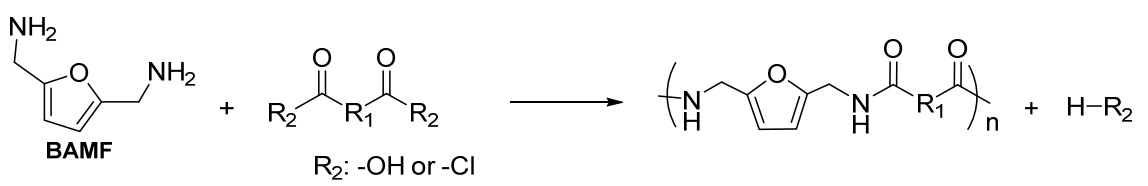

Figure 11. Polyamide synthesis from BAMF

Gandini et al successfully copolymerized BAMF in the presence of 2,5-bis(carboxyl chloride)furan. ${ }^{65}$ However, polyamides with low molecular weights were obtained. Moreover, the yield was found not to exceed $60 \%$. This was attributed to side reactions occurring onto the $\mathrm{CH}_{2}$-groups attached to the furan ring. A patent application from Rhodia also claims the preparation of polyamides from BAMF. ${ }^{66}$ In the process, a stoichiometric mixture of BAMF is performed in the presence of a diacid to yield the corresponding carboxylic ammonium salt. Polyamides are finally obtained thanks to a melt polymerization process.

\section{iv. Poly(imines / Schiff Bases) \& Polyvinyls}

As for polyamides, quite few examples of furan-based poly(imine) and poly(vinylene)s are encountered in the literature. Most of them describe the use of 2,5-diformylfuran (DFF) as a precursor (Figure 12). ${ }^{5,67}$

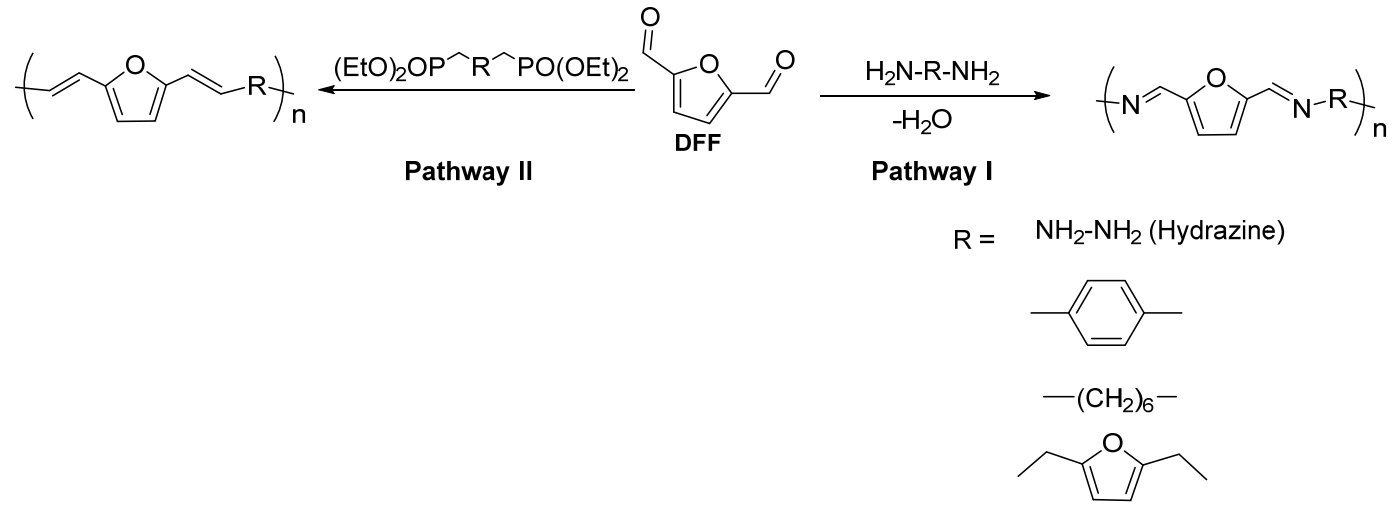

Figure 12. Synthesis of poly(imines) [pathway I] and poly(vinylene)s [pathway II] from 2,5-Diformylfuran (DFF)

As far as the formation of poly(imine)s is concerned, several diamines - namely 1,4-phenylenediamine, 1,6-diaminohexane, 2,5-bis(aminomethyl)furan (BAMF $)^{68}$ and hydrazine ${ }^{69}$ were used to investigate the structure-properties relationship. In the case of the three first diamines, low molecular weight, soluble polyimines were obtained $\left(\mathrm{M}_{\mathrm{n}}\right.$ were in the range of 1500-2500 g.mol $\left.{ }^{-1}\right)$. It is however to be noted that interesting thermal stability and optical properties were observed, in particular when 1,4phenylenediamine was utilized as a co-monomer. Such results were mostly attributed to the conjugation of the resulting polymer.

A more detailed study was conducted with hydrazine (Figure 13) with interesting properties such as a good thermal stability (decomposition started at $270^{\circ} \mathrm{C}$ without any sign of melting) and a conductivity of $10^{-5}$ to $10^{-4}{\mathrm{~S} . \mathrm{cm}^{-1}}$ after doping. However, the obtained molecular weight was of similar fashion as opposed to the other diamines $\left(\mathrm{M}_{\mathrm{n}}\right.$ was of 2000 g. $\left.\mathrm{mol}^{-1}\right)$. Those encouraging - but not satisfactory results led to further attempts in order to increase the final molecular weights as well as foster the processability of such polymerization processes. 


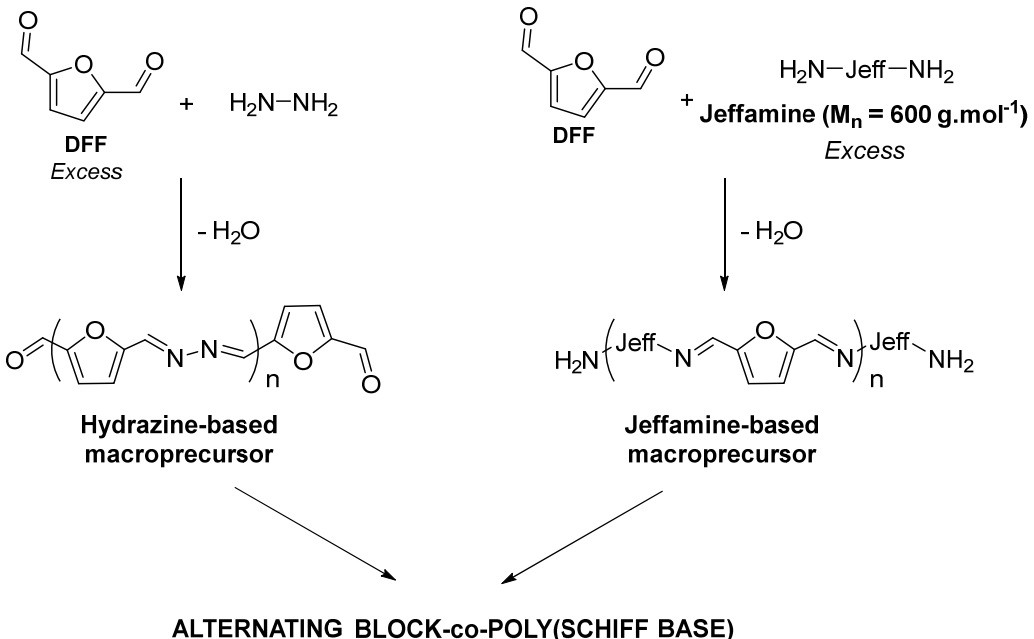

Figure 13. High molecular weight furanic Poly(Schiff bases) by block-copolymerization with soft segments

The introduction of soft segments in the polymer backbone was thus performed (Figure 13). ${ }^{69}$ The addition of Jeffamine $\left(\mathrm{Mn}=600 \mathrm{~g}^{\mathrm{mol}} \mathrm{mol}^{-1}\right)$ in a 2-step process thus successfully yielded a plastic-like soluble multiblock co-polymer with a molecular weight close to $20000 \mathrm{~g} \cdot \mathrm{mol}^{-1}$.

As far as the formation of poly(vinylene)s is concerned, reductive homocoupling reactions of DFF did unfortunately not yield polymers of interesting chain-length; the formed polymers were too insoluble to undergo further reaction. A solution was proposed by using the Horner-Wadsworth-Emmons Wittig coupling of phosphonate esters with aldehydes (Figure 13, Pathway II). Thanks to a wise selection of bifunctional aromatic phosphonate linkers, it was possible to form more soluble polymers with increased molecular weights along with interesting electronic properties thanks to the conjugation. ${ }^{70-72}$

\section{v. A reversible polymerization via a Diels-Alder process}

In a context in which the concepts of sustainability, durability and recyclability are emerging, it seems important to mention the growing use of the Diels-Alder reaction (DA) for the formation of thermally reversible macromolecular architectures. Such a system has gained increased attention due to the possibility to form re-crosslinkable, re-mendable, re-shapable and finally recyclable polymers.

The thermoreversible furan/maleimide system in particular perfectly applies to lignocellulosic-based polymers DA processes (Figure 14).

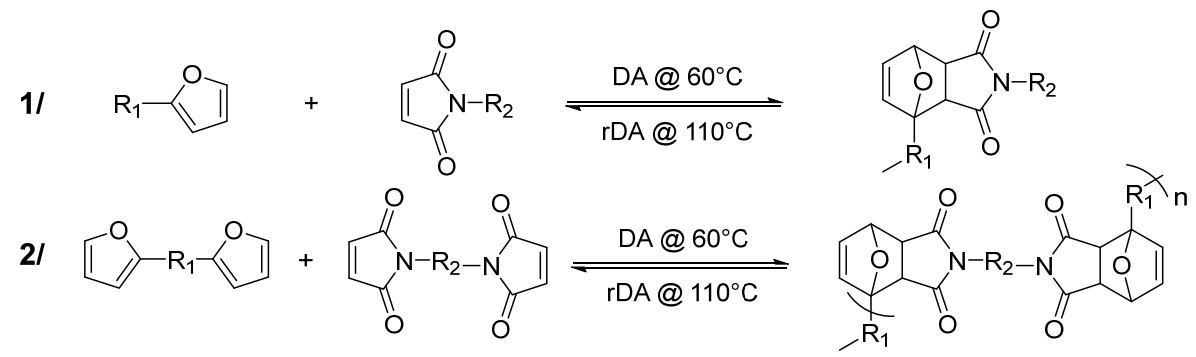

Figure 14. Equilibrium between the Diels-Alder reaction (DA) and retro Diels-Alder reaction (rDA) of $1 /$ furan- and maleimide- based monomers and of $2 /$ bis-furan- and bis-maleimide- based monomers

The furan/maleimide relies on the equilibrium between the DA reaction, occurring at a reaction temperature around $60^{\circ} \mathrm{C}$ and its reverse retro-DA reaction (rDA), occurring at the temperature around $110^{\circ} \mathrm{C}$. Both the temperature gap between the two reactions as well as the relatively low temperature range in which both events occur made them popular among the polymer community since it allows 
synthesizing thermally responsive and recyclable polymers without degradation. This allows for instance for the design of materials capable of self-healing.

Several pathways to incorporate the furan moieties have been presented in the scientific literature: the incorporation of the furan moiety in the inner-backbone of the polymer chain, the modification of an existing monomer/polymer prior to polymerization via a DA reaction or the formation of a DA reactionbased monomer prior to polymerization via another pathway (Figure 15).

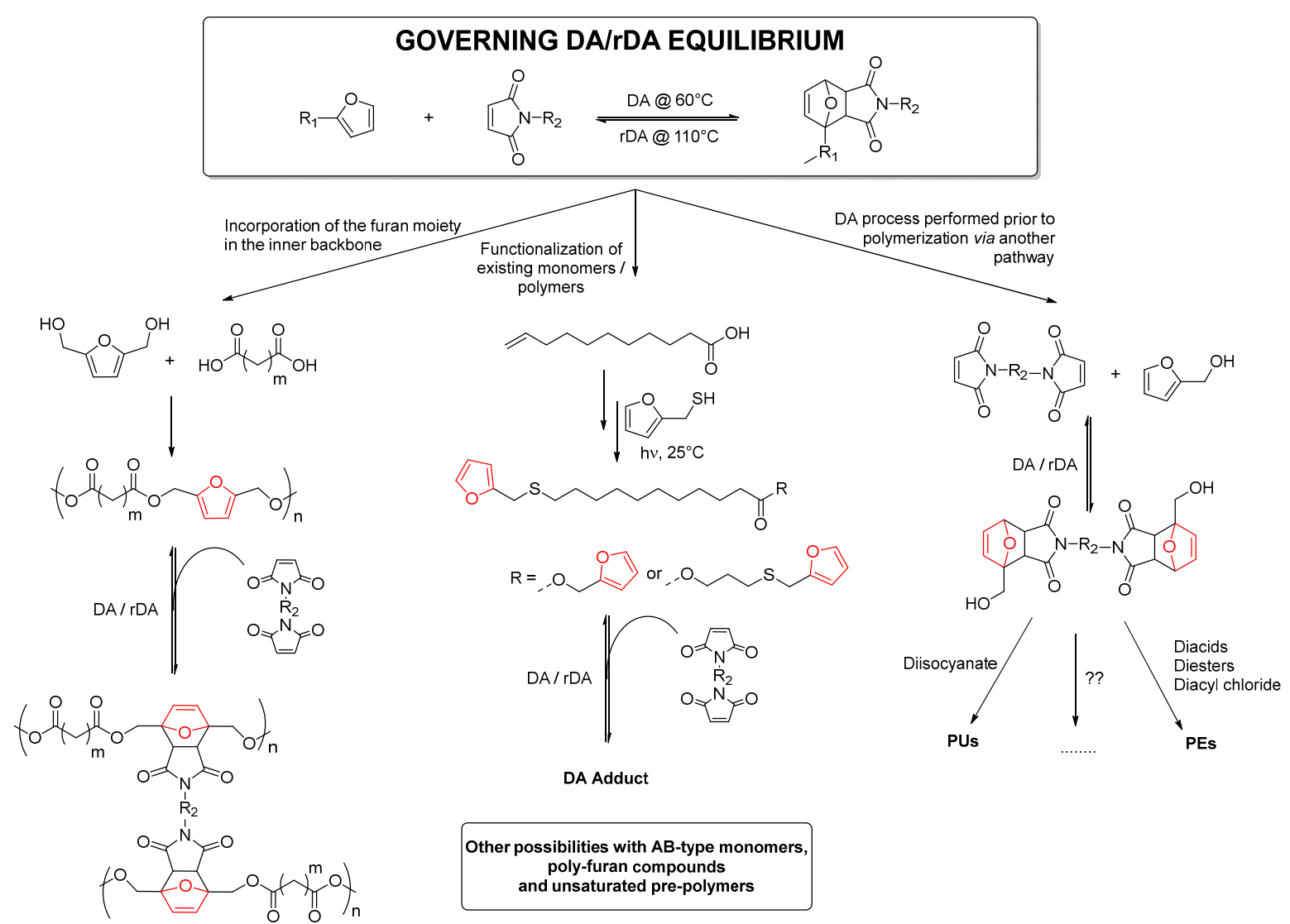

Figure 15. Several chemical pathways using the furan/maleimide DA system upon polymerization

Yoshie et al. studied the effect of incorporating the furan moiety in the inner backbone of polyesters prior to cross-linking via a DA process with a bis-maleimide-derived compound. ${ }^{42,43}$ The polyesters were synthesized by reacting together BHMF with succinic acid before cross-linking. Several bis-maleimides were tested and it was discovered that mendability was achievable, even with furyl telechelic prepolymers. $^{73}$

The team of Gandini used thiol-ene chemistry on vegetable oil-derived compounds in order to attach 2furylmethanethiol (obtained from FF) and thus a bio-sourced reactive diene for further DA/rDA process. Several strategies for making AA-type or AB-type monomers, targeting linear polymers, were investigated. The effect of increasing the functionality of the starting materials $\left(\mathrm{A}_{3}\right.$-type, $\mathrm{AB}_{2} / \mathrm{A}_{2} \mathrm{~B}$-type monomers, see Figure 16), then yielding branched polymers, was also studied to yield in fine a broad spectrum of polymers with various molecular weights and thermal properties. ${ }^{74,75}$ 


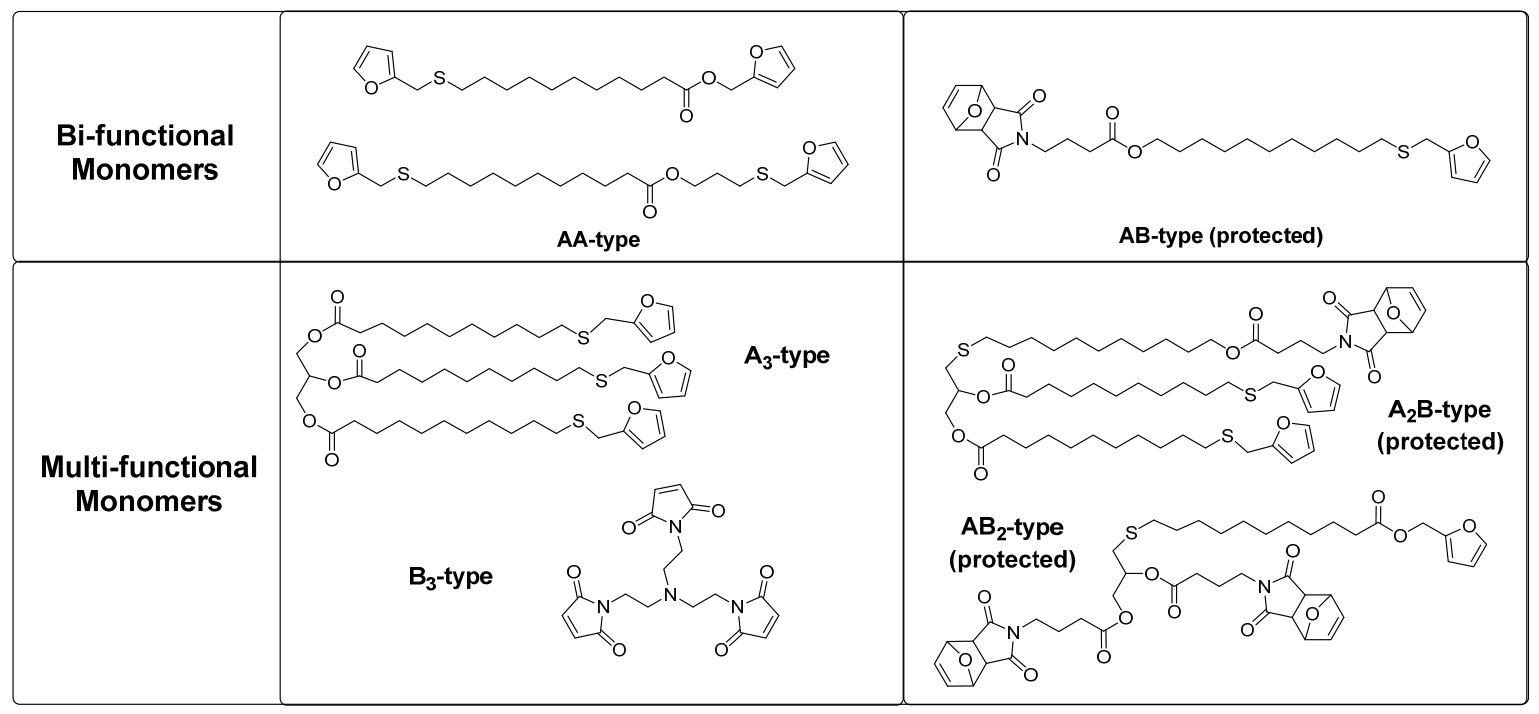

Figure 16. Different furan- and/or maleimide-based monomers with different functionalities as proposed by Gandini et al. ${ }^{74,75}$

Finally, it is worth mentioning the possibility to form a diol by means of reacting bis-maleimide with two equivalents of FF-OH (Figure 15). This monomer can thus be polymerized into a lot of polymers depending on the utilized copolymer (PUs and polyesters were reported). ${ }^{76}$ Very interestingly, the authors reported the possibility to play with the $\mathrm{DA} / \mathrm{rDA}$ equilibrium in order to form original and quite controlled polymer hybrids, thanks to a simple heating-then-cooling process. When PUs and polyesters were blended together and heated to allow for the rDA to proceed, the polymer chains were sliced and then recombined each other upon cooling to form PU-Polyester hybrids thanks to the DA reaction. Let alone the easy process at stake (successive temperature ramps), the full potential of such a process lies in the possibility to control the polymer sequence thanks to the kinetics of the rDA/Da processes. Varying the heating and/or cooling times made possible the control of the extent of depolymerization/repolymerization so that the scope of achievable products (and properties) becomes very large.

\section{B. Levulinic Acid-based polymers}

\section{Accessible monomers from Levulinic Acid}

Levulinic acid (LA) and levulinic acid-derived monomers are other kinds of very promising reagents. LA is earmarked by the DoE (see Figure 2) as one of the top value-added chemicals originating from biomass.

As described in Figure 17, levulinic acid can be obtained through the de-hydration process in acidic medium of HMF and FF. Once formed, it is then possible to convert this platform molecule into different other derivatives. Such derivatives have been extensively described by Isikgor and Becer ${ }^{77}$ but we will only focus on specific monomers in the course of this chapter. 


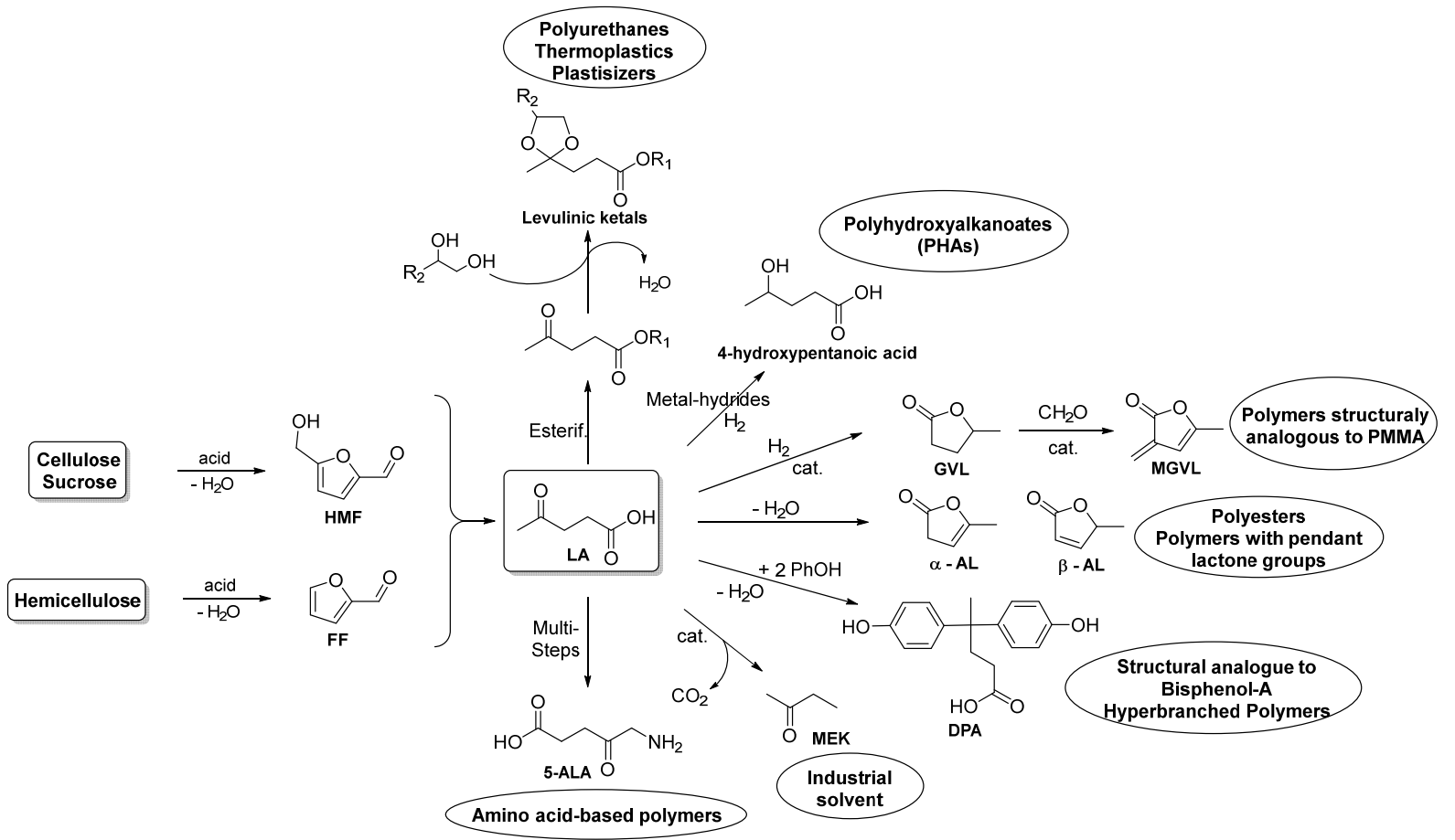

Figure 17. Possible derivatives from the levulinic acid platform and their use thereafter

It is interesting to notice that a large scale production of levulinic acid from lignocellulosic biomass has already been achieved. Maine BioProducts with its "Biofine Process" or Aventium's YXY technology claim to possess a large scale process for the production of levulinic acid. While the first is based on the catalyzed dehydration of lignocellulosic feedstocks into LA in a two-stage process ${ }^{78-81}$, the second company has developed a process to convert carbohydrates into high purity methyl levulinate. It is however to be noted that the cost-competitiveness of those production lines still has to be improved if a complete replacement of petroleum-based monomers is desired. Indeed, according to Becer, the current prices of LA remain between 5 and $8 \$ / \mathrm{kg} .{ }^{77}$ This price has to be decreased below $1 \$ / \mathrm{kg}$ to envision a cost-competitive process in which LA would play a key role in the replacement of fossil-based monomers. This constitutes one of the main challenges for researchers nowadays.

As exemplified in Figure 17, LA can be converted into various monomers, which correspond to diverse final applications. LA can be esterified then converted into levulinic ketal upon reaction with a diol (generally originating from vegetable oils). This process has been developed and led to an industrially viable process at Segetis. ${ }^{78}$ The corresponding products can be used for the production of polyurethanes and plasticizers. ${ }^{82}$

Amino-derivatives of LA can also be of high interest, in particular when it comes to their use in photomedicine. ${ }^{83}$ However, if the synthesis of 5-aminolevulinic acid (5-ALA) is possible at the laboratory scale by means of bromination agents, it remains a challenge to form 5-ALA at an industrial scale, because a safer and cheaper pathway has to be found to allow for a viable industrial process. ${ }^{77,84}$

2-butanone (methyl ethyl ketone - MEK) is accessible via a catalyzed decarboxylation step of LA. Among others, MEK is known for its use as a common industrial solvent. ${ }^{85}$

4-hydroxypentanoic acid can be obtained by means of a reduction reaction with hydrogen. This monomer is part of the hydroxyalkanoates family and can yield after polymerization the corresponding polyhydroxyalkanoate (PHAs). The reader willing further details about this kind of polymers is welcomed to refer to section 5 . 
Three other monomers: $\alpha$-angelica lactone (AL), $\alpha$-methylene- $\gamma$-valerolactone (MGVL) - also known as $\gamma$-methyl- $\alpha$-methylene- $\gamma$-butyrolactone and finally diphenolic acid (DPA), are particular targets from this platform and their polymerization will specifically be addressed in the next paragraphs. The latter are the most studied LA-derived monomers in the literature.

$\gamma$-valerolactone (GVL) can be obtained in high yields through a catalyzed hydrogenation process. Ruthenium-based catalysts were found to be the most suitable for this reaction. ${ }^{86,87}$ Unfortunately, the difficulty to find cheap catalysts or to recover the expensive ones prevents the implementation of GVL as a commodity chemical. GVL can thus be converted into MGVL. In 2004, Manzer et al. demonstrated that GVL could be reacted with formaldehyde yielding MGVL. This monomer has attracted interest due to its ability to substitute the common fossil-based methacrylate monomers. Two main processes were described: a continuous-gas-phase process using supported catalysts ${ }^{88}$ and a two-step cascade process requiring supercritical or near-supercritical $\mathrm{CO}_{2}$ as a solvent, the second process yield $90 \%$ of MGVL. ${ }^{89}$

Angelica lactones (AL) are dehydration products of LA in presence of acidic catalyst. Acetic anhydride or phosphoric acid were found to be suitable for this purpose. ${ }^{90,91}$ Finally, diphenolic acid (DPA) can easily be obtained thanks to the reaction of one mole of LA with two moles of phenol at $100^{\circ} \mathrm{C}$ under acidic conditions. ${ }^{84,92}$ Another pathway, using ionic liquids was also reported in 2013. ${ }^{93}$

\section{Polymers from Levulinic acid platform}

Different polymerization processes emerge from the LA platform. The structures of AL and GVL (and their derivatives) provide them versatility in the way of reacting: either by ring-opening polymerization (ROP) or by reaction of alkene moieties contained in or attached to the ring. Finally, DPA is a very particular monomer of interest due to its structure, very close to the chemical structure of bisphenol-A (BPA). This similarity made scientists think it could be a promising candidate for a bio-sourced replacement of BPA.

\section{i. Ring Opening Polymerizations}

GVL has been studied in processes involving ring-opening as exemplified in Figure 18. 


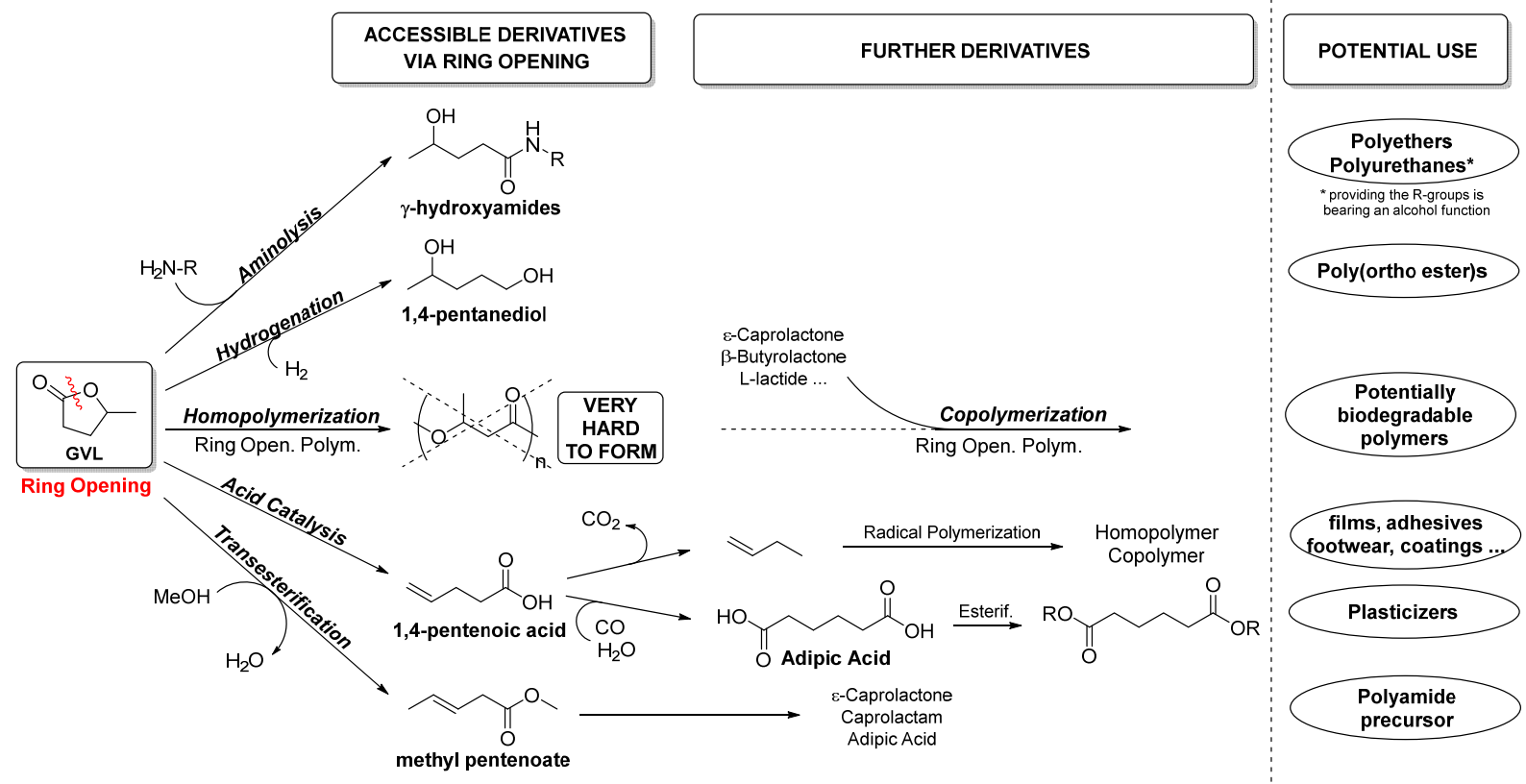

Figure 18. Possible derivatives from the $\gamma$-valerolactone platform and their use thereafter

GVL is a very stable 5-membered ring. This explains why to our knowledge, ring opening homopolymerization (ROhP) of GVL has not been described. This can be compared to its 6-membered ring counterpart, $\delta$-valerolactone, which has been successfully reacted through ROhP. It is often desired to pay careful attention to literature data as confusion might exist between these two monomers.

Ring Opening Copolymerization (ROcP) of GVL was thus performed in view of forming potentially biodegradable bio-sourced polymers. For instance, the copolymerization of GVL with diglycidyl ether of bisphenol-A (DGEBA) yields semi-bio-sourced thermosets. ${ }^{94}$ Copolymers of GVL and $\beta$-butyrolactone were also synthesized through a catalytic process. ${ }^{95}$

ROcP is a very interesting solution to force the opening of GVL. This process is however challenging and it prevents as a consequence the amount of available literature in the field. For this reason, researchers investigated other possible pathways to take advantage of this platform molecule. Quite an extensive work has thus been conducted in searching for suitable ways of ring-open GVL prior to polymerization. By opening the ring, the thermo-dynamical stability of the obtained intermediates - such as gammahydroxyamines, 1,4-pentanediol, 1,4-pentanenoïc acid and methyl pentenoate - is reduced and allows for further chemical modifications such as polymerization. Without trying to be exhaustive in the amount of presented examples, herein will be presented several occurrences of interest in the literature (Figure 18).

Thanks to an aminolysis process, GVL can ring-open to form $\gamma$-hydroxyamides. This process has been earmarked for its versatility to yield monomers that can be employed to produce relatively high molecular weight polymers, especially polyurethanes. Novel monomers can indeed be formed in high yields, in particular when non-sterically hindered amines were utilized in the ring-opening process. ${ }^{96}$ When aminoalcohol is used (for instance 1,2-aminoethanol), polyurethane could be formed by reaction with a diisocyanate.

By hydrogenation process thanks to a copper-based catalyst, GVL can yield 1,4-pentanediol. ${ }^{97}$ There is unfortunately scarce information about any polycondensation process of this monomer. The synthesis of poly(ortho ester)s has though been investigated in view of application in the pharmaceutical field. ${ }^{98}$ 
GVL can also be ring-opened with the help of a transesterification process. Lange et al. published in 2007 a transesterification process of GVL with a yield of $95 \%$ in which methanol is fed in a catalyzed reactive distillation process. ${ }^{99}$ Based on the different boiling points between GVL $\left(207^{\circ} \mathrm{C}\right)$ and methylpentanoate $\left(127^{\circ} \mathrm{C}\right)$, the latest is progressively isolated through successive distillation processes to yield a product as pure as possible.

Among the accessible monomers from levulinic acid, $\alpha$-Angelica Lactone (AL) can also be ring-opened. As described for GVL, the small ring strain energy of this 5-membered ring monomer inhibits its ROP. This is the reason why the successful homopolymerization of AL to the corresponding polyester is noteworthy to mention (Figure 19).

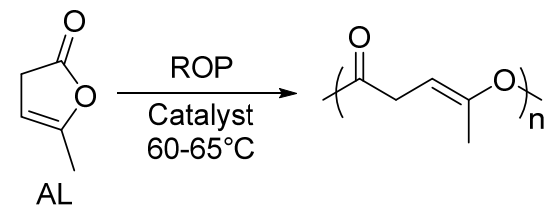

Figure 19. Ring-Opening homopolymerization of $\alpha$-angelica lactone

Lewis acid needs to be used to induce the ROP of AL. Several examples can be found in the Literature, such as sodium isopropylate. ${ }^{100}$ Polyester molecular weights were measured around $20 \mathrm{~kg} . \mathrm{mol}^{-1}$ after reaction at $60-65^{\circ} \mathrm{C}$ for $40-60 \mathrm{~min}$. It is also interesting to mention that 20 to $32 \%$ of the $\mathrm{C}=\mathrm{C}$ double bonds reacted during the ROP process. It is assumed that they got involved in a radical reaction process, which could account for this disappearance. Stannous octoate was employed in toluene at $130^{\circ} \mathrm{C}$ for $30 \mathrm{~h}$ for the ROP of AL, yielding a corresponding polyester with a $\mathrm{M}_{\mathrm{n}}$ of $29 \mathrm{~kg} \cdot \mathrm{mol}^{-1}{ }^{101}$

However, the presence of the double bond makes the final polyesters quite unstable, especially when exposed to daylight. The latter can also decompose in acidic or basic conditions. Thus, postfunctionalization of the AL-homopolymer via the chemical modification of the $\mathrm{C}=\mathrm{C}$ bond seems highly desirable if up-scaling of such processes are envisioned.

\section{ii. Polymerization through reactive alkene moieties}

Some LA derivatives contain reactive double bonds that can be reacted to yield original polymers. Among them, $\alpha$-methylene- $\gamma$-valerolactone (MGVL) has attracted a lot of interest as it could be a potential bio-sourced candidate for the replacement of methyl methacrylate (MMA). As described in Figure 20, MGVL can be synthesized through the reaction of GVL with formaldehyde. Due to the difficult handling of formaldehyde in the gas phase, it was found that dissolving GVL in formalin $37 \%$ aqueous formaldehyde) in the presence of Barium-based catalysts supported on Silica could be a suitable solution for the production of MGVL. ${ }^{88}$

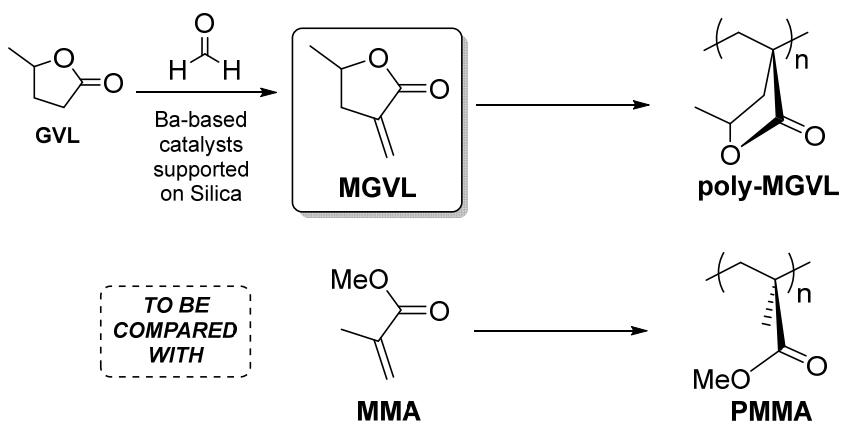

Figure 20. Radical homopolymerization of MGVL as a potential bio-sourced replacement for PMMA 
Early work focused on the polymerization of MGVL in free-radical emulsion process, radical, anionic and group-transfer polymerizations. However, those mostly led to incomplete conversion of MGVL. ${ }^{102}$ Chen's group published a series of papers tackling radical polymerization of MGVL using catalysts and provided extensive solutions through the use of frustrated Lewis pairs. In particular, alane-based frustrated Lewis pairs were found to be particularly suitable to promote the radical polymerization of MGVL. ${ }^{103-110}$ Fast processes could thus be developed with polymerization in the range of 10 minutes at room temperature. PolyMGVL molecular weights up to $500 \mathrm{~kg} \cdot \mathrm{mol}^{-1}$ could be reached when adapting the reaction conditions. As far as thermal properties are concerned, polyMGVL exemplified enhanced properties as opposed to PMMA with a glass transition temperature of $225^{\circ} \mathrm{C}\left(120^{\circ} \mathrm{C}\right.$ higher than PMMA) and with similar degradation temperatures. Resistance to heat, solvent and scratch could also be observed. ${ }^{111}$ This is attributed to the presence of the ring, providing more rigidity. However, it is to be noted that the obtained polymers were atactic and amorphous. Today, major challenges rely in finding both an economically attractive catalytic parthway to form polyMGVL as well as mastering the control of the tacticity of the final product to fully understand its influence on the resulting mechanical properties.

\section{iii. Diphenolic Acid-based Polymers}

Main examples of polycarbonates are based on the reaction of ester forms of Diphenolic Acid (DPA). DPA has attracted a lot of attention due to its similar structure with bisphenol A (BPA) making it a potential bio-sourced alternative to the petroleum-based monomer. As described in Figure 21, DPA is produced when reacting LA with two moles of phenol, thus forming a tri-functional compound containing two alcohol and one carboxylic acid moieties. Following the example of BPA, DPA was reacted to form polycarbonates. This however requires the acid group to be protected to avoid branching upon reaction.

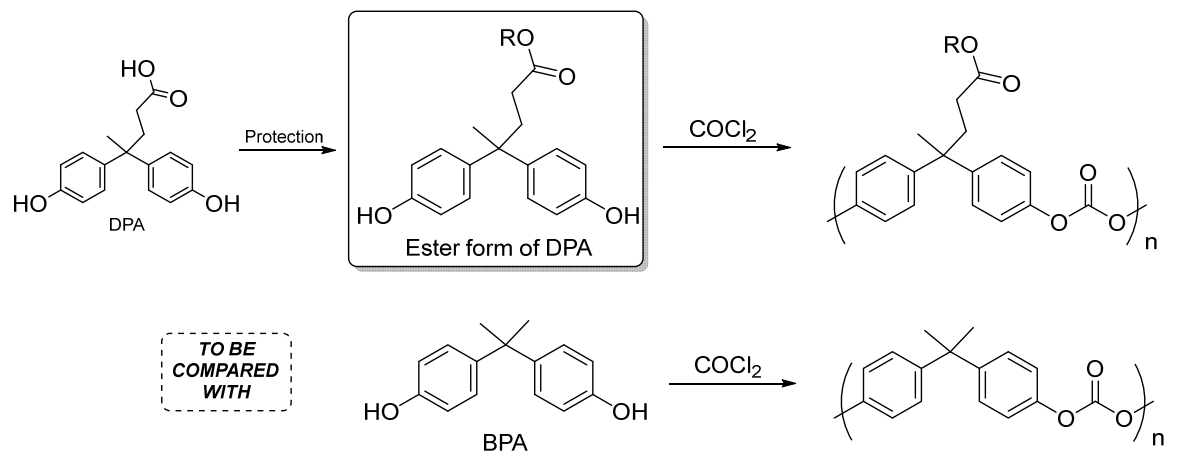

Figure 21. Formation of DPA-based polycarbonates

Polycarbonates formed with ester-DPA and phosgene were described in the literature. The final polycarbonate can be formed thanks to several chemical pathways. For instance, interfacial polymerization, using phosgene in gas phase and ester-DPA in a pyridine solution, or the use of phasetransfer catalysts gave interesting results. Polymers containing the ester form of DPA noteworthy exhibited similar molecular weight (around $140 \mathrm{~kg} / \mathrm{mol}$ ) and dispersity as opposed to their BPAcontaining counterparts. As far as physical properties are concerned, ester-DPA-containing polymers have lower glass transition temperatures than BPA-containing polycarbonates $\left(108^{\circ} \mathrm{C}\right.$ versus $\left.150^{\circ} \mathrm{C}\right)$. EsterDPA-based products were also soluble in organic solvent. It is finally interesting to mention that if the ester function is hydrolyzed, a dramatic change of the glass transition temperature can be observed as it increases up to $148^{\circ} \mathrm{C}$. This behavior was attributed to the formation of hydrogen bonds, inhibiting flexibility of the global system. ${ }^{112-114}$ 
DPA can also be used to form membranes. Zhou and Kim produced for instance sulfonated poly(arylene ether ketone) (SPAEK) using this monomer in the formulation. The pendant carboxylic acid groups were then used as crosslinkers to form crosslinked SPAEK membranes that exhibited promising properties when compared to the industrial Nafion ${ }^{\circledR} 117 .{ }^{115}$

Gross and co-workers have also reported the formation of bio-based thermosets through the synthesis of DPA-derived bis epoxy monomers, and their subsequent curing (Figure 22). ${ }^{16,117}$ By means of esterprotection of the acid moiety with short alkyl-chains, the addition of glycerol-devired epichlorohydrin under alkaline conditions formed diglycidyl ethers of diphenolate esters. Tuning of the monomer viscosity could be observed by varying the chain length of the alkyl chain of the ester moieties. Bio-based isophorone diamine was used to cure the so-formed bis epoxy monomers. Thermal and mechanical properties exhibited similar valued as opposed to "classical" DGEBA-derived thermosets, thus making DPA-based resins promising alternatives for petroleum-based epoxy-systems.

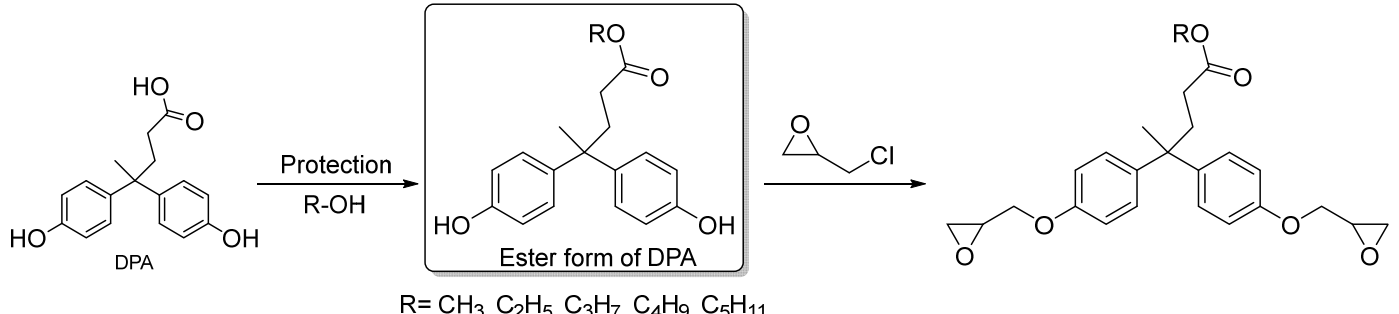

Figure 22. Synthesis of diphenolate ester-based glycicyl ethers

Hyperbranched polymers (HPBs) nowadays are gaining growing attention due to both their specific properties and their ability to be quite easily transferable at an industrial scale. The synthesis of HPBpolymers requires multifunctional monomers (at least tri-functional). This can allow for one-pot syntheses via intermolecular reactions. The obtained polymers, because of their highly branched architecture, usually have interesting physic-chemical properties, lying in between those of linear polymers and dendrimers. DPA contains a carboxylic acid moiety as well as two phenol units. It thus raises as an interesting and promising candidate for the production of HPB polyesters through $\mathrm{AB}_{2}$-type intermolecular polyesterification (Figure 23).
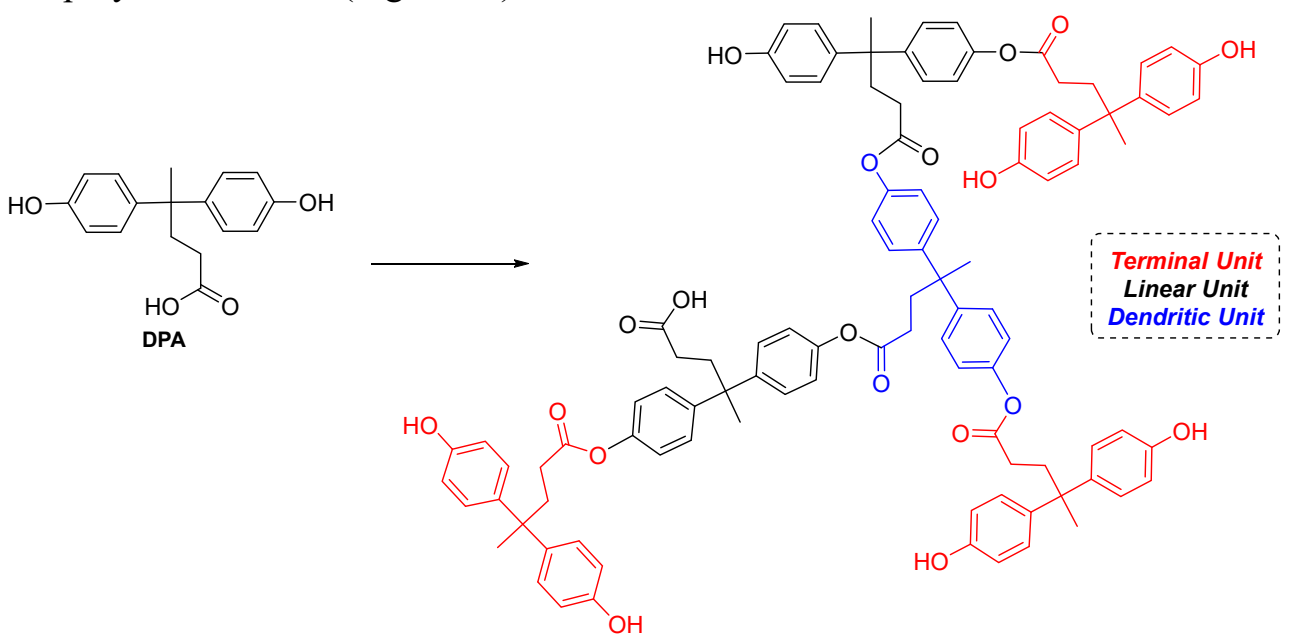

Figure 23. DPA-based hyperbranched polyesters

DPA-based HPB have been synthesized thanks to a melt condensation process, under reduced pressure with the help of catalysts. It is to be noted that a good control of the reaction temperature is needed (temperature ramps are used). Mw values ranging from 47 to $128 \mathrm{~kg} \cdot \mathrm{mol}^{-1}$ were obtained. No cyclization was observed during the reaction. The obtained polyesters were amorphous, with a $\mathrm{Tg}$ around $100^{\circ} \mathrm{C}$ and a degree of branching close to $49 \% .{ }^{118,119}$ Properties of the DPA-based HPB polyesters can also be tuned 
by means of copolymerization. As an example, Serra's team published in 2012 a DPA-Polyethylene glycol (PEG)-based triblock HPB copolymer, with a degree of branching of 50\%, Mn values of 10 $\mathrm{kg} . \mathrm{mol}^{-1}$ and self-assembly properties in solution of acetone. The same group used those compounds as chemical modifiers for photo- and thermal-curing of epoxy resins. ${ }^{120}$

\section{Isohexide-based polymers}

Multiple mono-saccharide-based monomers can be used in the preparation of polymers having sugar units incorporated into the main chain. These monomers named alditols, $\mathrm{HOCH}_{2}-\left(\mathrm{CH}_{2} \mathrm{OH}\right)_{n}-\mathrm{CH}_{2} \mathrm{OH}$, aldonic acid, $\mathrm{HOCH}_{2}-\left(\mathrm{CH}_{2} \mathrm{OH}\right)_{n}-\mathrm{COOH}$ or aldaric acid, HOOC- $\left(\mathrm{CH}_{2} \mathrm{OH}\right)_{n}-\mathrm{COOH}$ (Figure 24) were reported for the synthesis of polyesters, polyamides, polycarbonates and polyurethanes as recently reviewed by Galbis et al. ${ }^{121}$

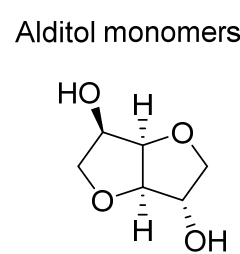

A<smiles>OCC1OCO[C@@H]2C(CO)OCO[C@H]12</smiles>

B<smiles>CO[C@H]1COC(=O)[C@@H](OC)[C@H]1OC</smiles>

Aldaric monomers<smiles>O=C(O)C1OCO[C@@H]2C(CO)OCO[C@H]12</smiles>

D<smiles>O=C(O)C1OCO[C@@H]2C(C(=O)O)OCOC12</smiles>

Figure 24. Examples of hexose-based monomers

Herein we will mainly focus on isosorbide (A, Figure 24) and derivatives also called isohexides and polymers thereof. Isosorbide and its stereoisomers, isomannide and isoidide (Figure 25) are thermally stable. The first two are currently commercially available and isoidide can be obtained from isosorbide. ${ }^{122}$ By chemical modification of these diols, isohexide-based dicarboxylic acid, ${ }^{123}$ diamine $^{124}$ and diisocyanate ${ }^{125}$ can be produced. The most important feature of these monomers is linked to their rigidity, chirality and non-toxicity. However, the latter exhibits relatively low reactivity due to the nature of secondary alcohol moiety.

Among isohexide derivatives, isosorbide is the most studied monomer. It does not possess any symmetry contrary to isomannide and isoidide and its two alcohols do not possess the same reactivity. ${ }^{126}$ Indeed the hydroxyl in $\mathrm{C} 3$ position exhibits an endo configuration generating a weak hydrogen interaction with the intracatenar oxygen of the connected ring (isomannide alcohols are in this configuration, too) while the configuration of the hydroxyl in $\mathrm{C} 6$ position is exo and thus more accessible (isoidide alcohols have also such a configuration), see Figure 26. 
Isosorbide can be obtained by double dehydratation of D-sorbitol or directly from starch or more recently from cellulose (Figure 25). ${ }^{127}$ Isomannide is synthesized in similar conditions from another highly available sugar, i.e. D-mannitol. Isoidide, which possesses the most reactive alcohol functions, can be produced from L-idose but the latter rarely exists in Nature and thus remains nowadays an expensive monomer. Routes need to be developed to isomerize sugar to L-idose in order to decrease its prize.

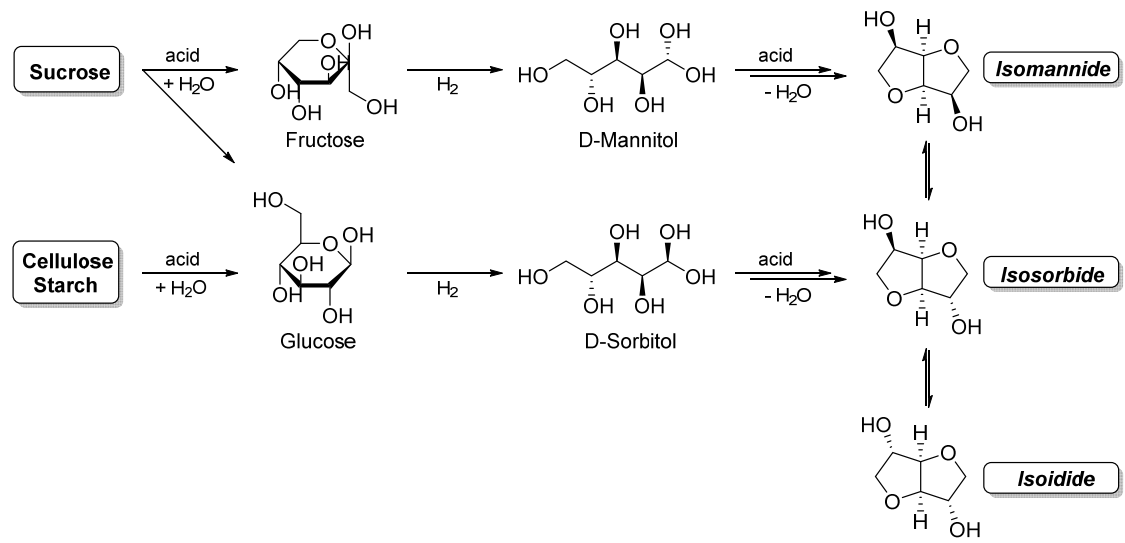

Figure 25. Molecular structures of isosorbide, isomannide and isoidide

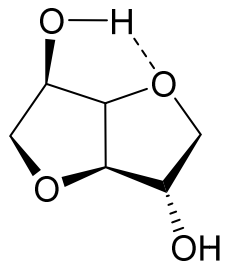

Figure 26. 3D molecular structure of isosorbide

The most representative polymers synthesized from these 3 monomers are polyesters, polycarbonates, polyethers and polyurethanes. They generally exhibit high $\mathrm{Tg}$, good thermomechanical resistance, have specific optical properties and can be biodegradable. Another important industrial application of isohexides is their use as diesters as substitutes to phthalate plasticizers. ${ }^{128}$

Isohexide-based aliphatic polyester can be obtained by molten polymerization using various diesters or their corresponding diacylchlorides (Figure 27). As a general trend, polyesters with a methylene sequence higher than 6 are semi-crystalline except for isomannide-based polyesters that remain amorphous. ${ }^{129}$ Moreover, isoidide-based polyesters exhibit higher melting temperature due to the symmetry of isoidide. As an example, polyesters from sebacic acid possess a $\mathrm{Tg}$ of $0^{\circ} \mathrm{C}$ for all three isohexides and a melting temperature of $60^{\circ} \mathrm{C}$ and $130^{\circ} \mathrm{C}$ for isosorbide and isoidide, respectively. ${ }^{130}$ Accordingly, the enzymatic degradation of polyesters based on isohexides and sebacic acid decreases in the order isosorbide $>$ isomannide $>$ isoidide. ${ }^{131}$

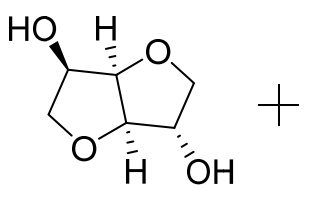<smiles>O=C(Cl)CC(=O)Cl</smiles>

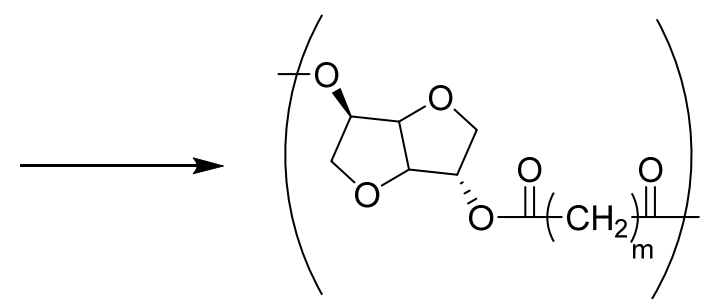

Figure 27. Synthesis of polyesters based on diacyl chloride and isosorbide

Isohexides can also be copolymerized with aromatic diacids. Poly(isosorbide terephthalate) (PIT), (Figure 28), exihibits a $\mathrm{Tg}$ of $200^{\circ} \mathrm{C}$ and its degradation temperature is over $360^{\circ} \mathrm{C}$; however limited polyester molecular weights are obtained due to the low reactivity of isosorbide. In the case of isoidide, a Tg of $210^{\circ} \mathrm{C}$ and a melting temperature of $260^{\circ} \mathrm{C}$ are obtained while, in the case of isomannide, only very low molecular weights are obtained. ${ }^{132}$ 


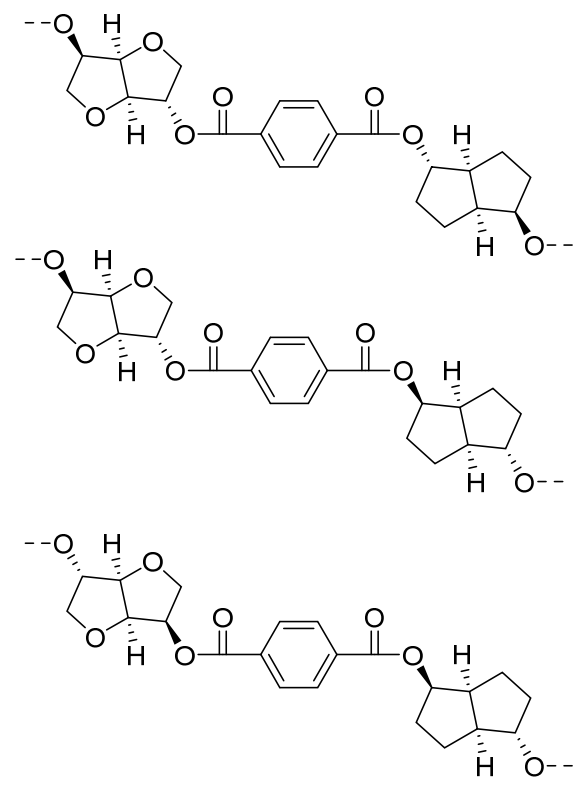

Figure 28. Chemical structures of isosorbide-based terephthalic acid-centered triads, PIT

One should note that PIT possesses 3 different stereo-sequences since the 2 hydroxyl functions are not equivalent and there is still a lack of report investigating the impact of the microstructure on PIT properties. Indeed, stereoregular PIT could exhibit even higher Tg and this polymer could compete with other high $\mathrm{Tg}$ thermoplastics such as poly(ether imide) for instance. Incorporation of isosorbide in a PET backbone (leading to PEIT) can increase the Tg of the polymer up to $+120^{\circ} \mathrm{C} .{ }^{133}$ PEIT and PIT are now allowed for food packaging and have the advantage to allow hot-filling contrary to standard PET. FDCA can also be copolymerized with isohexide leading to polyesters with $\mathrm{Tg}$ of 194,191 and $196^{\circ} \mathrm{C}$ for isosorbide, isomanide and isoidide, respectively. ${ }^{134}$

Another important class of polymers obtained from isohexides is the one of polycarbonates (Figure 29) as substitute to poly(carbonate of bisphenol-A). Poly(isosorbide carbonate), PIC, can be obtained by polycondensation of isosorbide with various phosgene analogs leading to a highly transparent polymer with a $\mathrm{Tg}$ around $170^{\circ} \mathrm{C} .{ }^{135}$

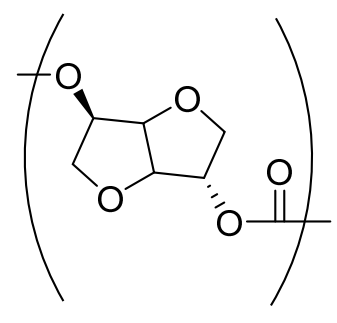

Figure 29. Poly(isosorbide carbonate), PIC

Similarly, copolycarbonates can be obtained, from 1/1 isosorbide/bis-phenol-A mixture. The polymers soformed have been reported with a $\mathrm{Tg}$ of $135^{\circ} \mathrm{C}$ and $160^{\circ} \mathrm{C}$, for the random and alternate copolymers, respectively. ${ }^{136}$ The insertion of isosorbide in a bisphenol-A polycarbonate is now commercially available as Durabioß. 


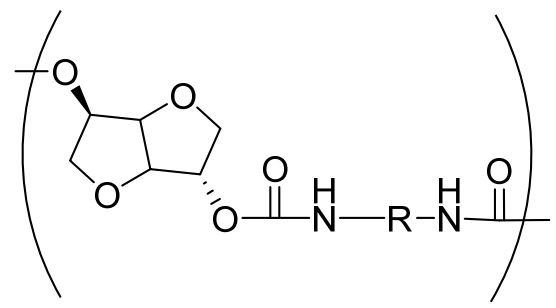

Figure 30. General structure of polyurethanes based on isosorbide as diol

As far as polyurethanes are concerned, isohexides can be used as hard segment diol. Isosorbide has been polymerized with various diisocyanates leading to polymers with $\mathrm{Tg}$ of $110^{\circ} \mathrm{C}, 135^{\circ} \mathrm{C}$ and $190^{\circ} \mathrm{C}$ for HDI, TDI and MDI, respectively (Figure 30). ${ }^{122}$ Isoidide gives similar Tg while isomannide leads to polyurethanes with a 20 to $50^{\circ} \mathrm{C}$ lower $\mathrm{Tg}$. Using diamino analog of isosorbide, fully isohexide-based polyurethanes can be synthesized. ${ }^{137}$ Low PU molecular weights were obtained (around $3000 \mathrm{~g} / \mathrm{mol}$ ) and $\mathrm{Tg}$ were in the range of $120^{\circ} \mathrm{C}$ to $135^{\circ} \mathrm{C}$ depending of the isohexide used.

\section{Synthons and/or polymers originating from biotechnological routes}

\section{Lactic Acid-based polymers (PLA)}

Poly(lactic acid), PLA, represents one of the most mature bioplastic as evidenced by its significant production and commercialization. ${ }^{138}$ PLA is a linear aliphatic polyester derived from lactic acid (2hydroxypropionic acid), a chiral organic hydroxyacid that exists in two different forms. Indeed, depending on the stereochemistry of the tertiary carbon of lactic acid molecule, the levogyre (L-lactic acid) and the dextrogyre (D-lactic acid) isomers are accessible. Lactic acid can be obtained by chemical synthesis or fermentation. Chemical synthesis of lactic acid is mainly based on the hydrolysis of lactonitrile by strong acids (Figure 31). Other routes are however suitable such as base-catalyzed degradation of sugars, oxidation of propylene glycol, reaction of acetaldehyde, carbon monoxide, and water at elevated temperatures and pressures, hydrolysis of chloropropionic acid and nitric acid oxidation of propylene. Main drawbacks of the chemical routes include the lack of cost effectiveness and the nonstereoselectivity. ${ }^{139}$ Indeed lactic acid produced by the petrochemical route exists as a 50/50 optically inactive mixture of L- and D- forms. Due to the above mentioned reasons and environmental concerns, the fermentative pathway to lactic acid has gained widespread interest and is now industrially developed by most of lactic acid producers. ${ }^{140}$

Indeed, the biotechnological production of lactic acid offers various advantages over the chemical pathway like low cost of substrates, low production temperature and low energy consumption. It is also noticeable that, depending on the micro-organism used in the biotransformation, L- or D- isomers are preferentially formed (Figure 31). ${ }^{141}$ Lactic acid bacterias (LAB) can be classified into two groups: homofermentative and heterofermentative. While the homofermentative LAB convert glucose almost exclusively into lactic acid, the heterofermentative $\mathrm{LAB}$ catabolize glucose into ethanol and $\mathrm{CO}_{2}$ as well as lactic acid. Only the homofermentative LAB are available for the commercial production of lactic acid. ${ }^{139}$ The carbon substrates used for microbial production of lactic acid include various sugars, either pure (e.g glucose and sucrose) or impure (e.g starch). Thus, a large variety of raw materials can be used such as molasses, sugar cane, bagasse and starchy materials from potato, tapioca, wheat, barley, corn 
etc. ${ }^{139,142,143}$ Starchy materials like sugarcane bagasse, cornstarch, corn cob, wheat bran are the most economical substrates for the production of competitive lactic acid.

By using such starchy substrates, several steps are needed to produce lactic acid. In conventional biotechnological processes, a pretreaTment step for gelatinization and liquefaction of the biomass is required, which involves the use of high temperatures in the range $90-130^{\circ} \mathrm{C}$. Then, enzymatic saccharification to glucose is carried out followed by subsequent conversion of glucose into lactic acid by fermentation. However, the conversion of starch or cellulose to sugar consumes energy during liquefaction or saccharification and increases the cost of production. In addition, sugar concentration in the hydrolyzate highly affects fermentation as bacterial cells cease to produce lactic acid when the sugar concentration is high. ${ }^{144}$ To overcome these limitations, direct fermentation processes were developed. The direct conversion of complex starchy or cellulosic substrates to lactic acid includes three different routes. In the first one, the lactic acid producing fungi can directly convert starch to lactic acid with the help of enzymes. In another pathway, amylolytic lactic acid bacteria allows direct fermentation of the substrate. ${ }^{144}$ The last possibility is to simultaneously treat the carbohydrate substrate with degrading enzymes and lactic acid producing bacteria. These solutions then offer the controlled release of sugar in the optimum growth temperature. Glucose inhibition on the enzyme is therefore minimized.

Due to considerable improvements, almost all the produced lactic acid is manufactured through fermentation routes. Thus current global production is between 300,000-400,000 tons per year with the United States being the largest regional market for lactic acid, accounting for a significant share of the worldwide market. Western Europe and Asia-Pacific make up the other major lactic acid producers and consumers on a global scale. The major manufacturers of fermentative lactic acid include Natureworks LLC (USA), Purac (Netherlands), Galactic (Belgium), Cargill (USA) and several Chinese companies.

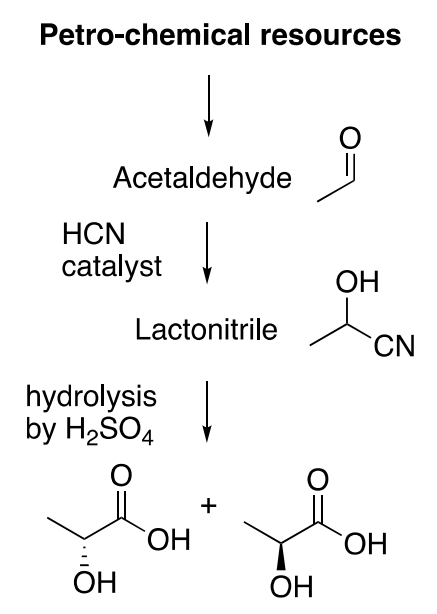

Racemic DL-lactic acid

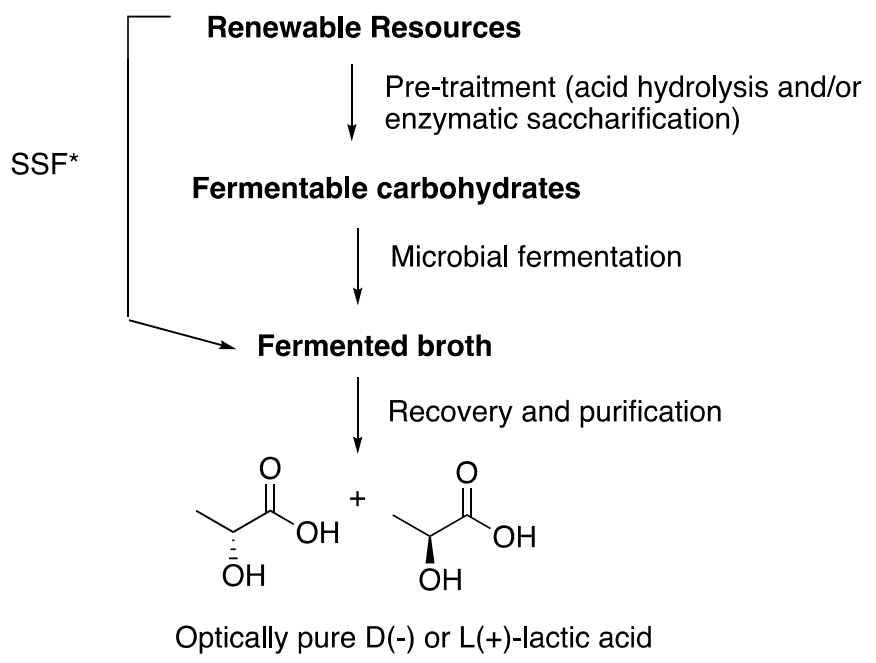

SSF*: Simultaneous Saccharification and Fermentation

Figure 31. Production of Lactic acid from petro-chemical resources or renewable resources (starch)

\section{i. Synthesis of PLA}

Two main routes are generally referred to convert lactic acid to PLA (Figure 32). Direct polymerization by polycondensation leads to poly(lactic acid) while the indirect route via lactide (LA), a cyclic dimer of lactic acid, leads to poly(lactide). Both products are referred to as PLA. ${ }^{145}$ Owing to the fact that lactic acid bears both a hydroxyl and a carboxylic acid function, polycondensation is inevitably the most direct route to PLA. However, molecular weights obtained by this pathway are not high enough to consider 
various applications of the resulting polymer. The low reactivity of the secondary hydroxyl function of lactic acid, combined with the need to efficiently removed water to shift the equilibrium toward the formation of the polymer constitute the main drawbacks of this pathway.

\section{Polycondensation route}

It is however possible to obtain higher molecular weights using different reaction conditions or via chain extension of pre-polymers. Indeed, a first strategy involves the polymerization of lactic acid in appropriate solvent that allows for azeotropic distillation of the condensate. ${ }^{146,147}$ Generally reaction time is in the range $30-40 \mathrm{~h}$ at $130^{\circ} \mathrm{C}$ making this process unsuitable for low cost PLA production. Moreover high concentration of catalyst is needed to have access to high molar mass PLA, which facilitates degradation and hydrolysis during processing.

Another approach to synthesize high molecular weight PLA is based on the polycondensation of lactic acid in the presence of difunctional monomers (e.g. diacids or diols) ending up in telechelic prepolymers. These resulting terminal functional groups of the prepolymers can then be reacted by using chain extenders such as diisocyanate, ${ }^{148}$ bis(amino-ether). ${ }^{149}$ However, increase in both cost and complexity of the multi-step process, as well as possible unreacted chain-extending agents, which alter the properties of the final material, are the main drawbacks limiting the industrial use of this method.

An alternative route consists in the melt/solid polycondensation of L-lactic acid. In this process, a polycondensate with a low molecular weight of around $20 \mathrm{~kg} \cdot \mathrm{mol}^{-1}$ is first prepared by ordinary melt polycondensation. Crystallization of the obtained poly(L-lactic acid) (PLLA) is then carried out by heattreaTment at around $105^{\circ} \mathrm{C}$ followed by heating at temperatures above Tg, yet below Tm of PLLA. Thus polymerization is performed in the amorphous phase, where all the reactive end groups reside, allowing the synthesis of high molar mass PLA in the range 100-500 kg.mol ${ }^{-1} \cdot{ }^{150}$

The advantages of solid-state polymerization include low operating temperatures, which control over side reactions as well as thermal, hydrolytic, oxidative degradations along with reduced coloration and degradation of the polymer. Moreover, there is practically no environmental pollution, because no solvent is required.

\section{PLA synthesis by Ring-Opening Polymerization (ROP) of lactide}

The most common way to obtain high molar mass PLA is through ring-opening polymerization (ROP) of lactide, Figure 32. The first step consists in the formation of the lactide, a cyclic dimer of lactic acid, by oligomerization of L-lactic acid, D-lactic acid or mixtures permitted by removal of the water condensate followed by subsequent catalytic depolymerization through internal "back-bitting" transesterification. 


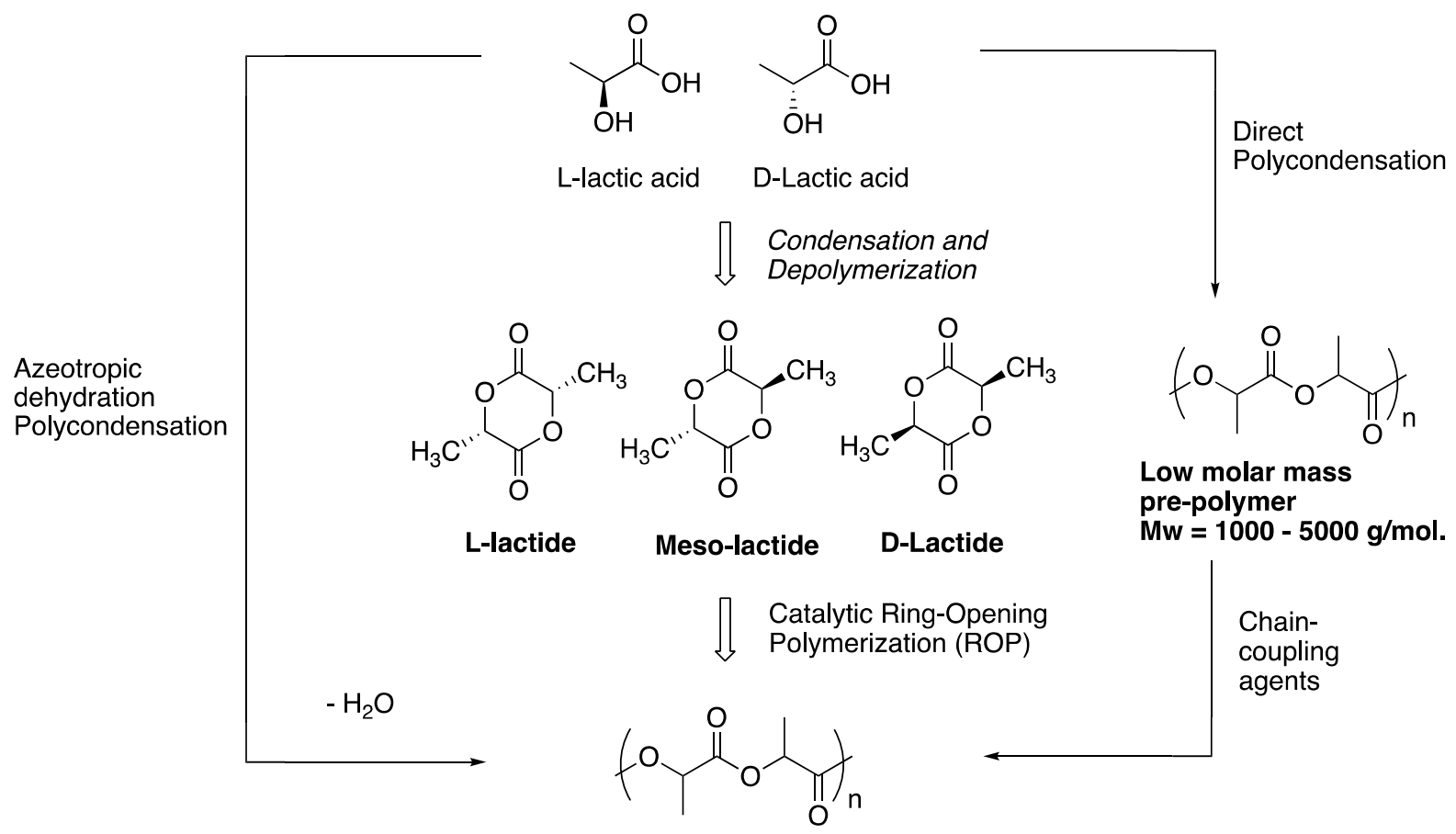

High molar mass Polylactide, PLA

Figure 32. Routes to high molecular weight PLA

Depending on the feed ratio of L-lactic acid and D-lactic acid, three stereoforms of lactide can be obtained: L-lactide (L-LA), D-lactide (D-LA) and meso-lactide (D,L-LA). The key point is the separation between each stereoisomer by vacuum distillation in order to control the final PLA structure. In a second step, the purified lactide is converted to high molecular weight poly(lactide) by catalytic ROP.

Polymerization through lactide formation is the current method used by most of PLA producers such as Natureworks, Purac, etc. The ROP of lactones is an attractive method to synthesize aliphatic polyesters because it enables living polymerizations to be conducted and therefore provides a route to control the physical and chemical properties of the polymers. The driving force of this polymerization method is the relief of ring strain. The polymerization mechanism involved in ROP can be anionic, cationic, coordination-insertion or an activated monomer mechanism depending on the initiating system used. ${ }^{151} \mathrm{~A}$ large range of catalytic species have been used to mediate ROP of lactide, including metal-based complexes, enzymes and small molecule organic catalysts. ${ }^{152-157}$

A large range of simple metal salts and coordination compounds have been reported as catalysts for the ROP of lactide (Figure 33). The catalysts mainly used consist of metal powders, Lewis acids, Lewis bases, organometallic compounds and different salts of metals. Particularly metallic compounds such as alkylmetals and metal halides, oxides and carboxylates are very effective. Among them, Tin(II) octanoate presents several advantages that made it a suitable catalyst for industrial production of PLA by ROP. ${ }^{158}$ It is soluble in organic solvents and molten lactide, is stable on storage, allows for polymerization up to $180^{\circ} \mathrm{C}$ and it has been approved by the food and drug administration (FDA). 


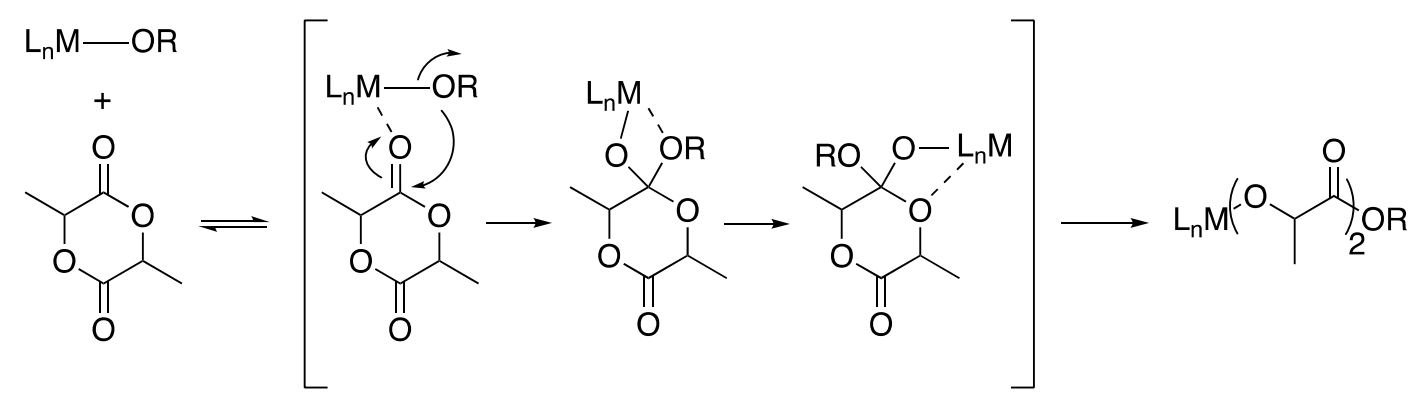

Figure 33. Coordination/insertion polymerization of lactide by metal-based catalysts

The applications of polyesters, and more particularly PLA, in packaging and biomedical applications have motivated efforts to develop metal-free organic catalysts in order to suppress the traces of metal in the final polymeric materials. The development of organic catalysts for the ROP of lactide has resulted in very high levels of polymerization control. ${ }^{152,159}$ Moreover, organic catalysts allow polymerization of lactide under milder conditions as evidenced by low temperature and reaction times needed. Organic catalysts are postulated to operate via one of the three mechanisms: monomer-activated, pseudo-anionic (chain-end activated or general base catalysis) or cooperative dual activation.

The particularity and potential of PLAs reside in the various stereochemical microstructures enabled by the presence of two stereocenters per lactide monomer (Figure 34). The ROP of enantiopure L- or Dlactide results in the isolation of isotactic PLA in which all stereocenters are identical. However, ROP of either rac-lactide (racemic mixture of L- and D-lactide) or meso-lacitide (lactide possessing both L- and D-stereocenter) leads to an atactic PLA if the utilized catalyst lacks stereospecificity. Some reviews summarize the different catalytic systems used to synthesize stereoregular PLAs. ${ }^{154,160}$ Two types of stereospecific catalysts can be distinguished. First, catalysts that display a syndio preference will result in the synthesis of heterotactic PLA -the latter will display doubly alternating stereocenters (i.e. LLDDLLDD-) from rac-lactide by alternating insertion of monomers of opposite stereochemistry or syndiotactic PLA (alternating L- and D- stereocenters i.e. -LDLDLD-) from meso-lactide. Second, catalysts that display an iso preference (i.e., a preference to ring-open at the same stereocenter as the one of the propagating polymer chain end) will result in the synthesis of heteroatactic PLA from meso-lactide but will mediate the synthesis of stereoblock PLAs from rac-lactide.

Special emphasis was made on isotactic PLLA due to its interesting mechanical and thermal properties provided by semi-crystallinity. Isotactic PLLA was preferred over isotactic PDLA due to the higher availability of L-LA from biomass in comparison to D-LA. 

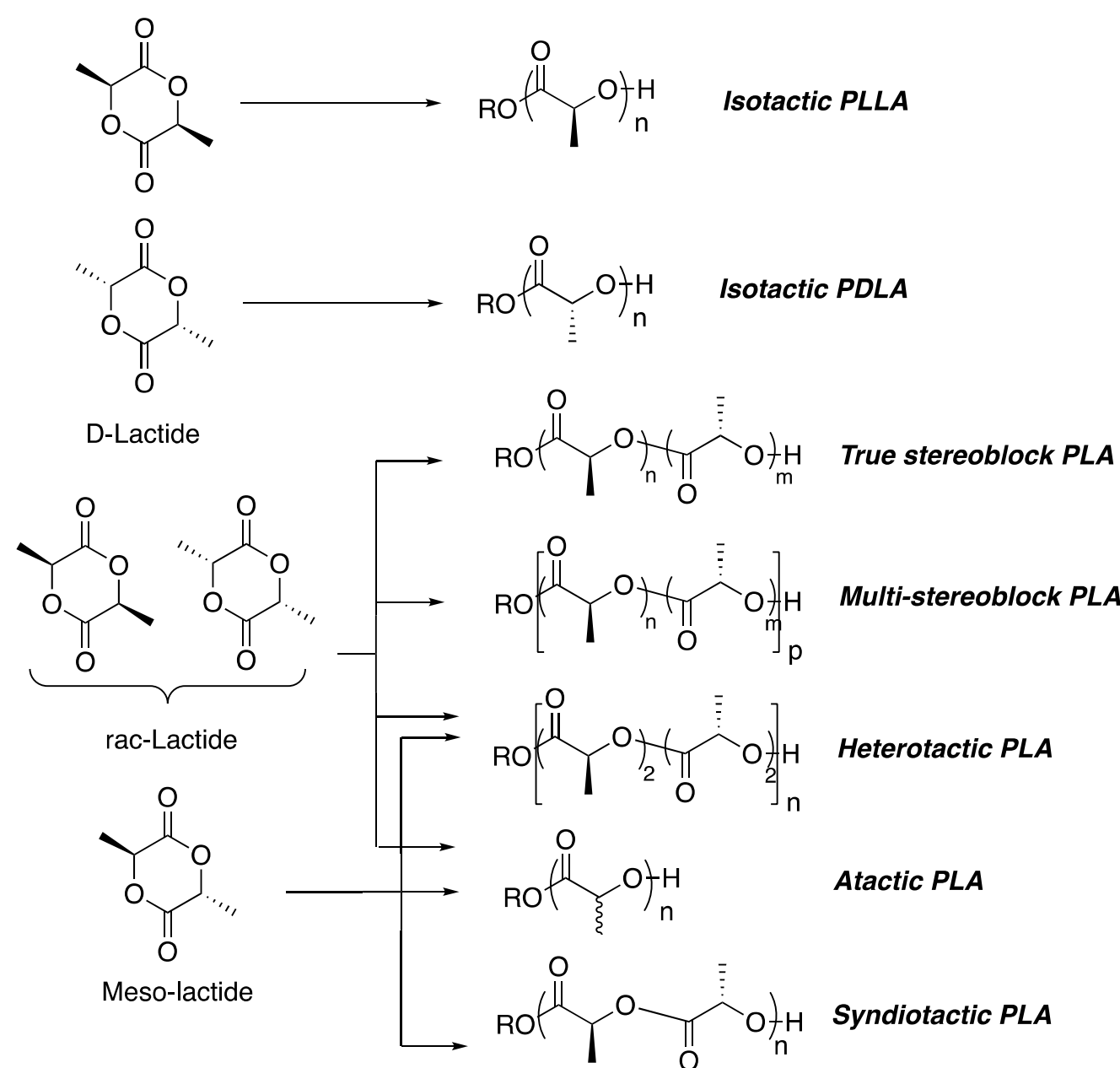

Multi-stereoblock PLA

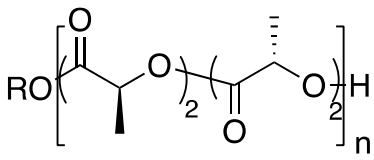

Heterotactic PLA<smiles>CC(=O)C(C)O</smiles>

Atactic PLA<smiles>[R]O[C@H](C)[C@@H](C)OC(=O)[C@H](C)O[TlH]</smiles>

\section{Syndiotactic PLA}

Figure 34. Stereochemistry of PLA materials

\section{ii. PLA properties and compostability}

\section{Structure, thermal and physical properties}

Due to the three forms of lactide monomers, various natures of PLA can be achieved. High purity L- and D-LA form stereoregular isotactic poly(L-lactide) (PLLA) and poly(D-lactide) (PDLA) respectively. Both are semi-crystalline polymers with a melting point of $207^{\circ} \mathrm{C}$ and a glass transition temperature in the range $55-60^{\circ} \mathrm{C} .{ }^{161,162}$ However typical melting points are in the range $170-180^{\circ} \mathrm{C}$ due to small and imperfect crystallites, slight racemization and impurities. The meso-lactide or a racemic mixture of L- and D-LA, on the other hand form atactic poly(D,L-lactide) (PDLLA) which is completely amorphous and shows a $\mathrm{Tg}$ in the range $50-60^{\circ} \mathrm{C}$.

Another family of polylactides includes stereocomplex-type polylactides (sc-PLA) consisting of both enantiomeric PLLA and PDLA. In 1987, Ikada and co-workers discovered that a mixture of enantiomeric PLLA and PDLA forms stereocomplex (or racemic) crystals with a melting temperature at around $240^{\circ} \mathrm{C}$. ${ }^{163}$ Stereocomplex-type polylactides were first generated from solution and later from melt mixtures. ${ }^{164,165}$ Various factors affect the stereocomplexation such as the molar mass of PLLA and PDLA, the optical purity of the homopolymers and the process of formation.

For amorphous PLA, the glass transition temperature (Tg) is one of the most important parameters since dramatic changes in polymer chain mobility take place around this temperature. Various physical properties are reached depending on the temperature. Below the $\beta$-relaxation temperature $(240 \mathrm{~K}), \mathrm{T}_{\beta}$, 
PLA is completely brittle. Between $T_{\beta}$ and $T g$, the amorphous PLA undergoes physical aging and can show brittle or ductile fracture. Above Tg, amorphous PLA is rubbery and becomes viscous in the range $110-150^{\circ} \mathrm{C}$. Finally, amorphous PLA decomposes between 215 and $285^{\circ} \mathrm{C}$.

For semi-crystalline PLA, physical properties are also highly dependent on the processing conditions. Indeed, the processing conditions will dictate the degree of crystallinity while the stereochemistry will govern the melting point of the polymer. Below Tg, semi-crystalline PLA is a brittle material while, above $\mathrm{Tg}$, it is tough and undergoes a ductile fracture.

\section{Crystallization behavior and crystallographic properties}

Isotactic PLA can crystallize in $\alpha$-, $\beta$-, or $\gamma$-forms depending on the processing conditions. ${ }^{16-169}$ The most common and stable, which can be developed from the melt or solution under normal conditions, is the $\alpha$ form with a $10_{3}$ helical chain conformation where two chains are interacting in an orthorhombic (or pseudo-orthorhombic) unit cell.

PLLA is a slow-crystallizing material with fastest rates of crystallization observed in the temperature range of $110-130^{\circ} \mathrm{C}$, which yields spherulitic crystalline morphology. However, it is difficult to make use of the semi-crystalline character of PLLA because the high speed of mass production (e.g. in injection moulding) implies quenching, leading to amorphous structures. One particularity of quenched amorphous PLA is to crystallize upon reheat. This phenomenon is allowed by the enhanced mobility of PLLA chains observed above Tg. This enhanced mobility allows reorganization and better packing of the chains, which leads to crystallization. However, similarly to the crystallization upon cooling, the cold-crystallization upon heating highly depends on the heating rate. The crystallinity can be either an advantage or a drawback depending on the targeted application. For instance, high crystallinity will not be optimal for packaging solutions due to lack of optical clarity while, in contrast, increased crystallinity will be desirable for injection moulded articles for which good thermal stability is important (related to the increase of heat distortion temperature (HDT) with crystallinity). The lack of crystallinity due to fast cooling in industrial PLLA articles can be overcome by several techniques. Post-process heating treaTment higher than the Tg and below the Tm can be carried out leading to improved flexural elasticity, Izod impact strength, and heat resistance. ${ }^{170}$

\section{Physical and Mechanical properties}

The specific gravity of amorphous PLA has been reported as $1.248{\mathrm{~g} . \mathrm{cm}^{-3}}^{-}$and for crystalline PLLA as 1.290 g. $\mathrm{cm}^{-3}$ which is lower than PET $\left(1.34 \mathrm{~g} . \mathrm{cm}^{-3}\right)$ but higher than many of other conventional

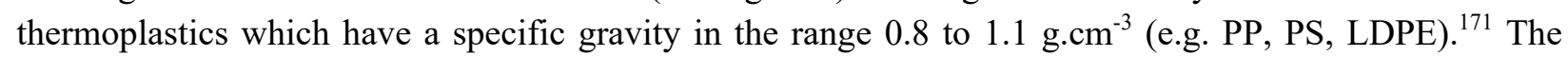
optical properties of PLLA are highly dependent to additives and fabrication effects. Indeed, a low degree of crystallinity will induce transparency while highly crystalline PLLA has poor optical properties, even if transparency can be preserved by reducing the crystallite size.

Similarly to the thermal properties, mechanical properties of PLA can be tuned to a large extent depending on the molecular weight, the stereochemical composition and the thermal history of the polymer. ${ }^{170}$ Semi-crystalline PLA is generally preferred to the amorphous counterpart when higher mechanical properties are desired. Perego and coll. demonstrated that the modulus of elasticity is almost $20 \%$ higher for PLLA than for PDLLA for samples having molar masses in the range $35000-55000$ g.mol ${ }^{-1} \cdot{ }^{170}$ In addition, the same authors showed that the impact strength was also strongly influenced by the crystallinity. Annealing PLLA increases impact strength due to crosslinking effects on the crystalline domains. ${ }^{9}$ Furthermore, high molecular weight PLA is generally needed to have access to suitable mechanical properties. 
Heat deflection is also a key parameter that needs to be underlined as one of the main drawbacks of PLLA. Poly(98\% L-LA) looses $50 \%$ of its elastic modulus and storage modulus at $80^{\circ} \mathrm{C}$ and $87 \%$ of the storage modulus at $100^{\circ} \mathrm{C}$. Above the glass transition, non-annealed PLLA has not sufficient film strength to target a wide range of applications. This lack of film strength at temperature above the $\mathrm{Tg}$ can be overcome by increasing the crystallinity of the material.

\section{Rheological properties and Processing}

Melt processing of PLLA materials is essential for the conversion into industrial products. Extrusion is the most important technique for continuously melt processing of PLLA. Extrusion is generally linked to a subsequent processing step such as thermoforming, injection molding, fiber drawing, film blowing or extrusion coating. From these, injection molding is the most widely used converting process for PLLA articles. The different processing technologies for PLLA were reviewed by Rubino and coworkers. ${ }^{147}$ The processing of PLLA is a crucial step in the formation of valuable PLLA in order to keep the structural, thermal and mechanical properties of the starting polymer. Indeed, PLLA has a narrow processing window due to its tendency to undergo thermal degradation in the molten state through many mechanisms (hydrolysis, zipper-like depolymerization, oxidative main-chain scission, intermolecular transesterification and intramolecular transesterifications). ${ }^{172}$

\section{Environmental degradation of PLA}

PLLA represents a promising polymer due to its compostable behavior (Figure 35). PLLA degradation occurs in two stages. First, high molecular weight PLLA is subject to non-enzymatic chain scission of the ester functions by hydrolysis. Second, low molecular weight PLLA is transformed to lactic acid and shorter oligomers that are naturally metabolized by microorganisms to yield $\mathrm{CO}_{2}$ and water.

Various factors have an influence on the biodegradation rate. Indeed, exposure conditions can be distinguished from the polymer characteristics. Concerning exposure conditions, moisture, acidity, temperature, aerobic or anaerobic conditions and enzyme specificity are the most influent factors. Other parameters have to be taken into account; i.e. the polymer microstructure that will dictate the chain flexibility and thus the accessibility of the ester functions to water, the crystallinity, the molecular weight, the residual lactic acid concentration and the size and shape of the polymer (surface area). More details about the influence of all these factors on the biodegradation rate can be found elsewhere. ${ }^{171,173-175}$

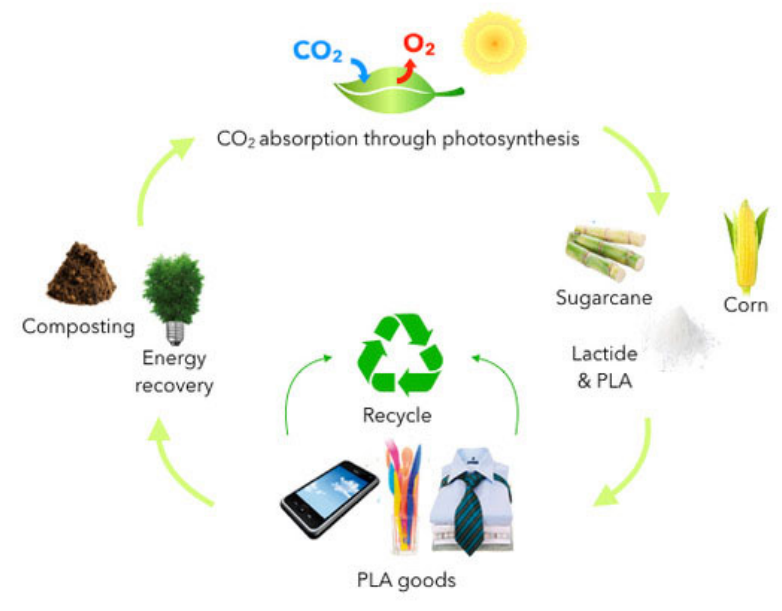


Figure 35. PLA life cycle.

Compostability and recyclability of PLLA imply an environmental friendly lifecycle, and the industrial are targeting a neutral carbon footprint. Indeed, PLLA presents a cradle-to-cradle lifecycle where products are produced from sustainable and natural resources. In this type of lifecycle, the polymer is used, re-used and recycled as much as possible. At their end-of-life, when the products do not satisfy their functional requirements anymore, these products are transformed back into feedstock for a new product lifecycle (Figure 35).

\section{iii. Summary of PLA development and remaining challenges}

PLLA is a very promising and versatile compostable polymer. One issue that restricts the development of PLLA in a wide range of applications seems to be its price (around $2 € / \mathrm{kg}$ ). However, this trend could be reversed in the next years due to improvement of biotechnological processes. Nevertheless, there are a number of areas in which PLA-based materials drawbacks still need to be overcome to consider efficient and feasible substitution of existing thermoplastics. For instance, in packaging applications where high barrier protection is essential for the conservation of food, efficient solutions need to be found as barrier properties of PLLA are not in par with those of PET or PP. Examples of current investigations deal with the addition of nanoparticles such as nanoclays. ${ }^{176}$ Another drawback that needs to be significantly improved is the enhancement of PLLA crystallization rate. Numerous solutions were already found; however, finding a highly efficient solution fitting with the high-speed mass production is still a challenge. The compostable character of PLLA represents an important advantage in environmental applications; nevertheless, aging studies related to the specific conditions of use still need to be conducted in a more precise way in order to anticipate the biodegradation rate. Another area that needs to be studied and improved is the energy and raw materials consumption of PLLA production and its impact on the environment. Related to this, Vink and coworkers published an interesting work presenting the life cycle assessment (LCA) of NatureWorks PLA. ${ }^{140}$ In particular, it was mentioned that Cargill Dow's objectives are to decrease the fossil energy use from $54 \mathrm{MJ} / \mathrm{kg}$ PLA down to about $7 \mathrm{MJ} / \mathrm{kg}$ PLA and to decrease the greenhouse gases emission from +1.8 down to $-1.7 \mathrm{~kg} \mathrm{CO}$ equivalents $/ \mathrm{kg}$ PLA. Finally, the inherent brittleness of PLLA is a major bottleneck for its use in applications where toughness and impact resistance are critical.

\section{Succinic acid-based polymers}

Succinic acid (SA) is an important chemical building block available from biochemical transformation of biorefinery sugars that can also be obtained by chemical transformation from bio-based molecules such as maleic acid and furfural, Figure 36. ${ }^{177,178}$ Bio-succinic acid and diesters thereof can be produced through fermentation using a wide range of bacterial strains including Actinobacillus succigogenes, Anaerobiospirillum succiniciproducens, Propprionibacterium succiniciproducens, Mannheimia succiniciproducens. ${ }^{179}$ Glucose and glycerol are the main carbon sources used but more recent developments using lignocellulosic biorefinery streams (e.g. wood hydroxylate) as carbon source are investigated. The strain efficiency is also $\mathrm{CO}_{2}$-dependent. Indeed, increasing $\mathrm{CO}_{2}$ concentration usually results in higher SA yields and reduces by-product concentration (lactate, ethanol). 


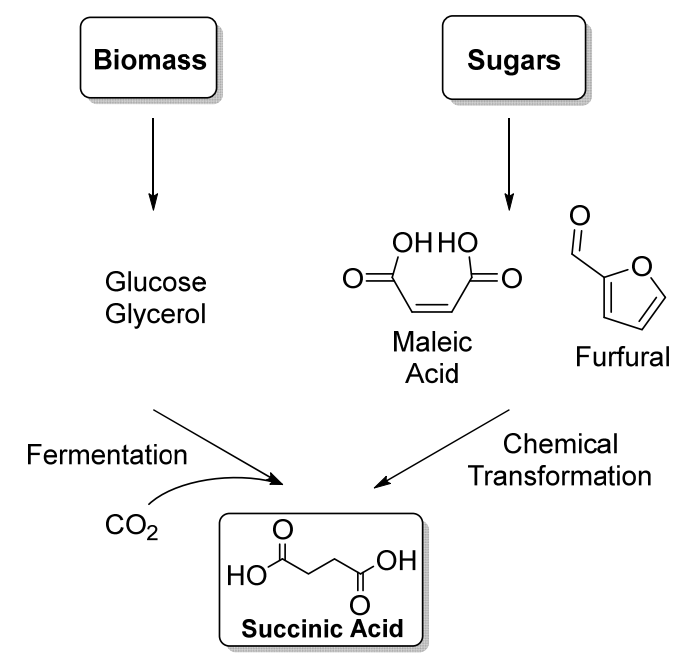

Figure 36. Conversion of biomass into succinic acid (SA)

Using Anaerobiospirillum succiniciproducens as the fermentative organism and a three-stage continuous cell recycle bioreactor, optimized processes producing $10.4 \mathrm{~g} . \mathrm{L}^{-1} \cdot \mathrm{h}^{-1}$ and a final concentration of $83 \mathrm{~g} . \mathrm{L}^{-1}$, equivalent to $1.35 \mathrm{~mol}$ succinic acid per mol of sugar, have been reported. ${ }^{180}$ Currently, four major commercial producers are active (BioAmber, Myriant, Succinity $\mathrm{GmbH}$ and Reverdia) using high grades glucose sources with a production price quasi equal to fossil-based SA, i.e. around $0.5 \$ / \mathrm{kg}$.

Succinic acid offers strong potential as a chemical platform for polymer synthesis (see Figure 37). ${ }^{5}$ Succinate esters are precursors for known petrochemical products such as 1,4-butanediol, 1,4-diaminobutane, tetrahydrofuran, $\gamma$-butyrolactone (GBL) or various pyrrolidinone derivatives (vinyl pyrrolidinone).

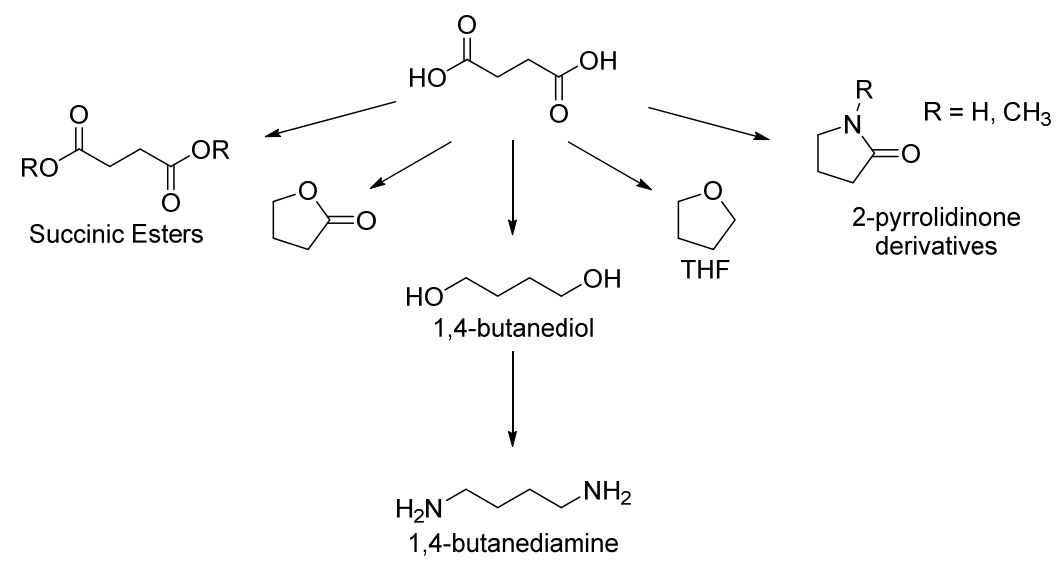

Figure 37. Derivatization of succinic acid into platform molecules and polymers thereof

From SA platform, various semi-crystalline polyesters (PEs) and polyamides (PA) can be produced. In particular, the copolycondensation of succinic acid with 1,4-butanediol and 1,4-diamino-butane has been reported. ${ }^{181}$

The so-formed aliphatic polyesters either based on succinic acid (PEs-n,4) or 1,4-butane diol (PEs-4,n) exhibit melting points ranging from $-24^{\circ} \mathrm{C}$ to $113^{\circ} \mathrm{C}$. PEs-4,4, or poly(butylene succinate) (PBS) and PEs2,4 are already commercialized due to their polyethylene-like behaviors combined with a good biodegradability. ${ }^{5}$ Moreover, copolyesters have also been investigated using two sets of diacids and or diols to tune their mechanical properties and degradability. ${ }^{182-184}$ In particular, copolyesters of ethylene glycol 
and 1-4-butanediol together with succinic and adipic acids are commercialized. ${ }^{185}$ It should be noted that high molecular weight polyesters are usually obtained in a two-step procedure starting first by the synthesis of a low molecular weight telechelic polyester diol followed by a chain extension using a diisocyanate (usually hexamethylene diisocyanate). ${ }^{186}$ In the case of 1,4-butanediol as a diol, poly(butylene terephtalate) (PBT) represents also a polymer of interest with its properties close to PET $\left(\mathrm{Tg}=60^{\circ} \mathrm{C}\right.$ and $\mathrm{Tm}=223^{\circ} \mathrm{C}$ ) with a good processability especially for fiber industry.

As far as polyamides are concerned, aliphatic polyamides either based on succinic acid (PA-n,4) or on 1,4-butanediamine (PA-4,n) exhibit high melting point $\left(\mathrm{Tm}>200^{\circ} \mathrm{C}\right)$. PA-2, $4^{187}$ and PA-4, $2^{188}$ exhibit excellent mechanical properties similar to natural silk and could be used as fibers. PA-4,6 and PA-4,10 are actually commercialized as high performance polyamides for automotive industry. ${ }^{181}$

Biodegradable polyesters based on succinic platform have attracted industrial attention as environmentally degradable thermoplastics. However, their thermal and mechanical properties are not optimal for some applications. The properties of such polyesters can be improved by the introduction of amide groups into the main chain. ${ }^{189}$ Different biodegradable poly(ester amide)s, PEAs, were commercialized. The synthesis is based on the amide-ester inter-exchange reaction that takes place when a polyester and a polyamide are heated together at a temperature close to $270^{\circ} \mathrm{C}$. PEAs based on PA-6 and PE-4,10 or PA-6,10 and PE-4,10 are the most investigated. ${ }^{190}$

Finally, other polymers can be obtained from the succinic platform. Poly(4-hydroxybutyrate), P4HB, and poly(tetramethylene oxide), PTMO, can be obtained from $\gamma$-butyrolactone ${ }^{191}$ and THF, respectively. Another and more efficient route to P4HB is the fermentation process (see poly(hydroxyalcanoate)s, section 4). Indeed, in the case of ROP of GBL, molecular weights are limited due to the low reactivity of the 5-member ring lactone. On the contrary, PTMO raised a lot of interest and more specifically as dihydroxy-telechelic PTMO, which is widely used as polyol in the course of thermoplastic elastomers.

\section{Itaconic acid-based polymers}

Similarly to succinic acid, itaconic acid (IA) has raised considerable interest in the last decade. Its chemical structure composed of two carboxylic acid moieties and a double bond, makes IA a promising precursor for a large platform of chemicals (Figure 38) that are structural isomers of levulinic acid-based derivatives: for instance, 3-methyl-THF can be obtained from IA in comparison to LA-based 2-methylTHF, same for $\delta$-valerolactone in comparison to $\gamma$-valerolactone. ${ }^{5,77}$ The reduction of the acidic functions of IA leads to 2-methyl-1,4-butanediol, which is an interesting diol towards the synthesis of polyesters and polyurethanes. ${ }^{192}$ Finally, the decarboxylation of itaconic acid to methyl methacrylate is of a huge interest for the industry. ${ }^{193}$

Nowaday IA can industrially be produced with a yield of 80 g.L $\mathrm{L}^{-1}$ via the fermentation of carbohydrates with fungi. However, the productivity remains at a low level, $1 \mathrm{~g} \cdot \mathrm{L}^{-1} \mathrm{~h}^{-1} .{ }^{194}$

IA is extremely attractive to polymer chemists for the production of a wide range of polymers by providing two orthogonal reactivities via its two carboxylic acid functions and its activated carbon double bond. Namely, IA can either react via step-growth polymerization (usually radical polymerization) or chain growth polymerization (polyesterification). 


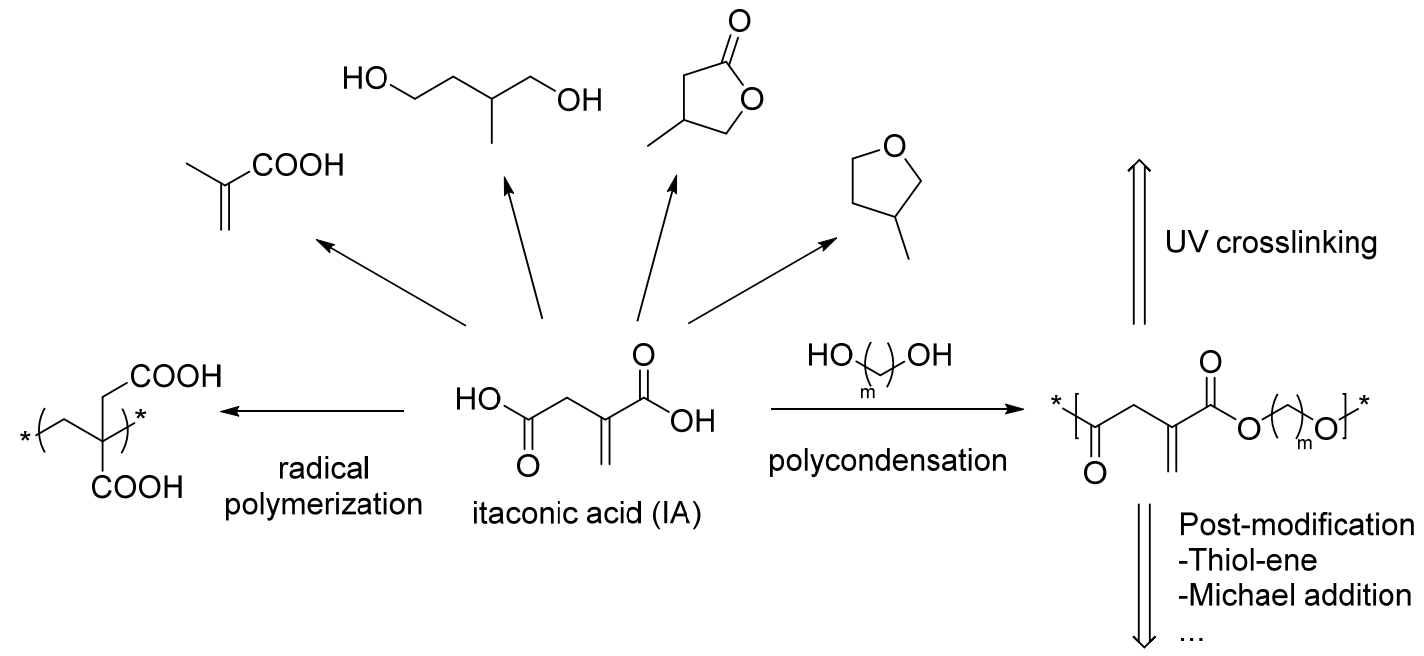

Figure 38. Main platform molecules from itaconic acid

\section{i. Chain-growth polymerization of Itaconic acid derivatives}

Due to IA similarity to acrylic acid and methacrylic acid, IA and the corresponding esters such as dimethyl itaconate, dibutyl itaconate and others have been intensively investigated as alternative (co)monomers in the production of poly(alkyl(meth)acrylate)s. The polymerization of itaconic acid and its diester derivatives has been reported since the 1970s via radical and anionic polymerization to produce relatively high molecular weight polymers. Their copolymerization with vinylic monomers was investigated in industry for long. ${ }^{195}$ Polymers of the dialkyl itaconates are similar in hardness to polymers of the corresponding alkyl methacrylates. As an example, poly(di-n-butyl itaconate) exhibits a $\mathrm{Tg}$ of $25^{\circ} \mathrm{C}$ compared to $29^{\circ} \mathrm{C}$ for poly(n-butyl methacrylate). Homopolymer of itaconic acid is commercially available as an equivalent compound of polyacrylic acid. ${ }^{194}$

Furthermore, itaconamide and itaconimide were also polymerized via anionic and radical routes. Poly(itaconimide) exhibits excellent thermal properties due to its cyclic structure $\left(\mathrm{Tg}>220^{\circ} \mathrm{C}\right) .{ }^{196}$

All these itaconate derivatives can also be synthesized by controlled radical polymerization. ${ }^{197}$ This allows producing bio-based acrylic thermoplastic elastomers. Triblock copolymers have been prepared by the sequential block copolymerization of itaconate and itaconimide, in that order, from di-functional RAFT agent, which produces chains with inner soft poly(itaconate) and outer hard poly(itaconimide) segments (Figure 39). The obtained triblock copolymers exhibit the characteristic properties of thermoplastic elastomers along with the microphase-separated morphology observed by AFM. ${ }^{198}$

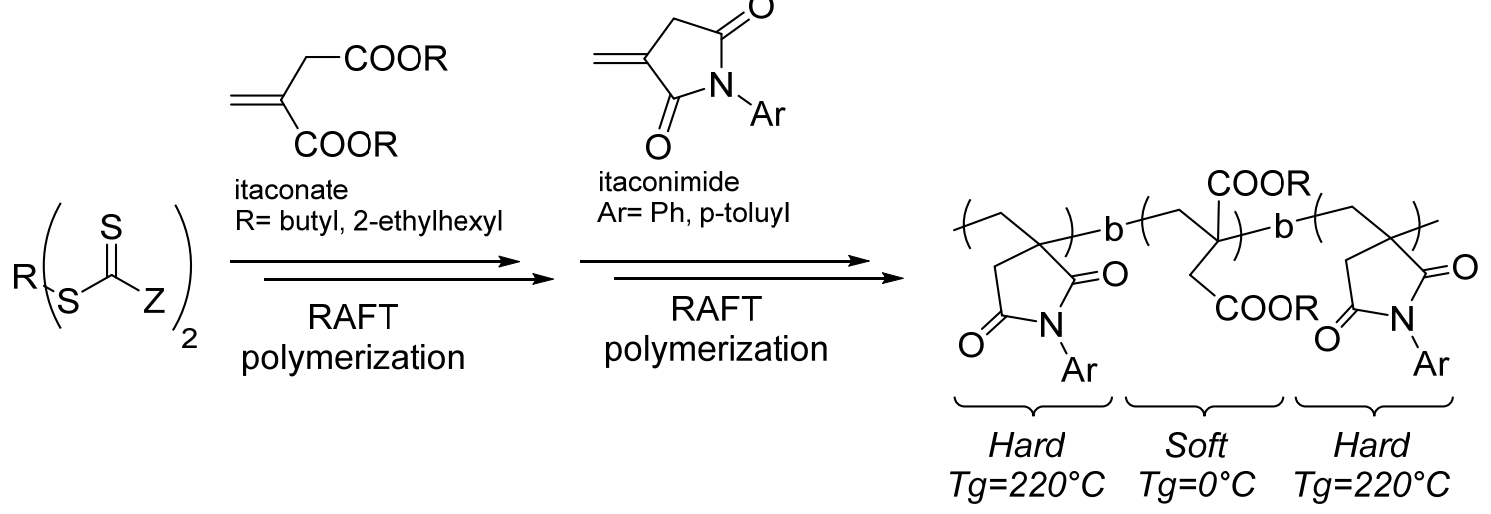

Figure 39. Itaconic-based triblock copolymer as acrylic thermoplastic elastomer 


\section{ii. Step-growth polymerization of Itaconic acid derivatives}

Polycondensation of itaconic acid received less attention in comparison to the chain-growth polymerization. ${ }^{199}$ The so-formed polymers are unsaturated polyesters, UPEs, with a wide range of applications thanks to their ability to undergo various post-polymerisation and/or cross-linking reactions (Figure 40). The latter can be utilized in high-gloss coatings, insulating materials, drug delivery systems and biomedical applications. A wide variety of diols have been incorporated into the polyester alongside the itaconate moiety such as: 1,4-butanediol, ethylene glycol, trimethylolpropane or isosorbide. Moreover, itaconic acid can be copolymerized with other di-acids (e.g., succinic, maleic or fumaric acids) allowing the tuning of the physico-chemical properties. The molecular weights of the obtained polyesters are usually relatively low. The synthesis of higher molecular weight by polycondensation has indeed been hampered by the tendency of the itaconate double bond to act itself as a cross-linker, presumably via Ordelt reaction. Radical inhibitors are often needed to enable efficient formation of even the smallest oligomers. ${ }^{200}$ Moreover, isomerization of the double bond usually takes place leading to ill-defined polyesters.

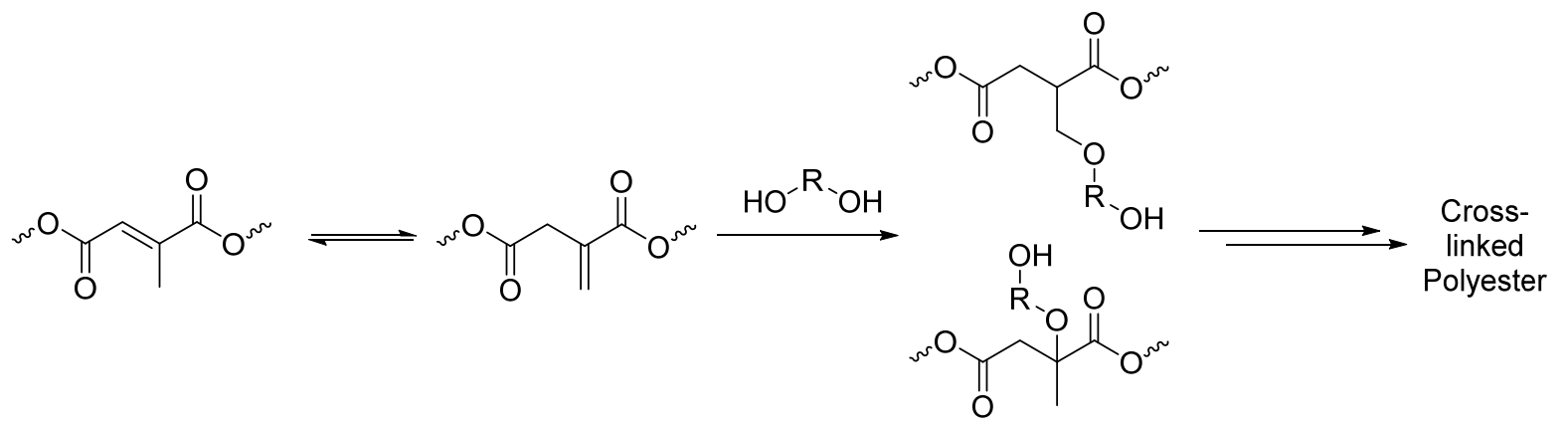

Figure 40. Side-reaction occuring during itaconate-based polyester synthesis

Polyesters based on itaconic acid are frequently modified through its exo double bond. Because of its conjugation with the adjacent carbonyl moiety, the vinyl group acts as a Michael acceptor and allows for post-polymerisation functionalisation and hence property tuning of the itaconate-based polyesters. These polyesters can be subjected to Micheal additions with several sulfur- and nitrogen-based nucleophiles including protected amino acids such as proline and cysteine, for biological applications. ${ }^{201}$ Interestingly, modification by primary amines leads to self-immolated polymers at temperature higher than $37^{\circ} \mathrm{C}$ via lactam formation. This thermally induced degradation of polyesters exhibits high potential for biomedical applications (Figure 41).

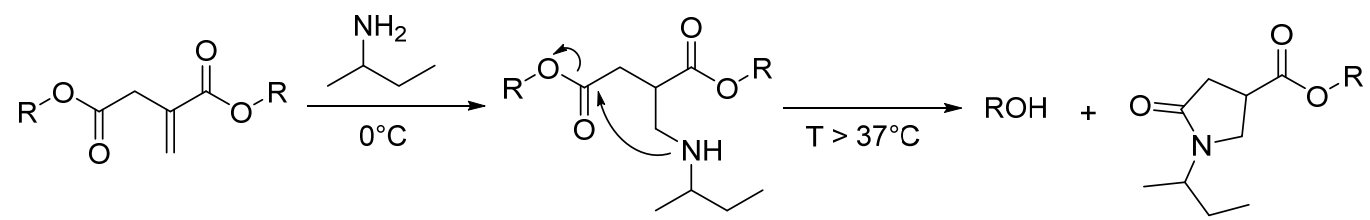

Figure 41. Self-immolated mechanism of itaconate-based polyester

Another usually employed strategy for itaconate-based polyester is its cross-linking via UV-curing leading to polyester thermosets. As an example, copolyesters of succinic acid, itaconic acid and butanediol can be crosslinked, leading to thermoset with Tgs ranging from $0{ }^{\circ} \mathrm{C}$ to $100^{\circ} \mathrm{C} .{ }^{202}$

Finally, it is noteworthy to mention that itaconic acid can be derivatized by epichlorydrin yielding epoxy resins $^{202}$ or by Diels-Alder reaction with cyclopentadiene for ROMP of norbornene derivative. ${ }^{203}$ 


\section{Poly(hydroxyalkanoate)s (PHAs)}

In contrast to main polymers, which are polymerized through standard routes starting from bio-based monomers, poly(hydroxyalkanoate)s, PHAs, are biogenic polyesters (Figure 42). PHAs can be naturally produced by bacteria to levels as high as $90 \%$ of the cell dry, in general cultivated on agricultural feedstock. Structurally, these polyesters are classified on the basis of the number of carbon atoms that ranges from 3 to 14 and the type of monomer units, producing homopolymers or heteropolymers. PHAs with 3-5 carbon atoms are considered as short chain length PHAs (scl-PHAs). Examples of this class include poly(3-hydroxybutyrate), $\mathrm{P}(3 \mathrm{HB})$ and poly(4-hydroxybutyrate), $\mathrm{P}(4 \mathrm{HB})$. Medium chain length PHAs (mcl-PHAs), contain 6-14 carbon atoms. Examples include homopolymers poly(3hydroxyhexanoate), $\mathrm{P}(3 \mathrm{HHx})$, poly(3-hydroxyoctanoate), $\mathrm{P}(3 \mathrm{HO})$ and copolymers such as $\mathrm{P}(3 \mathrm{HHx}$-co$3 \mathrm{HO}){ }^{204}$

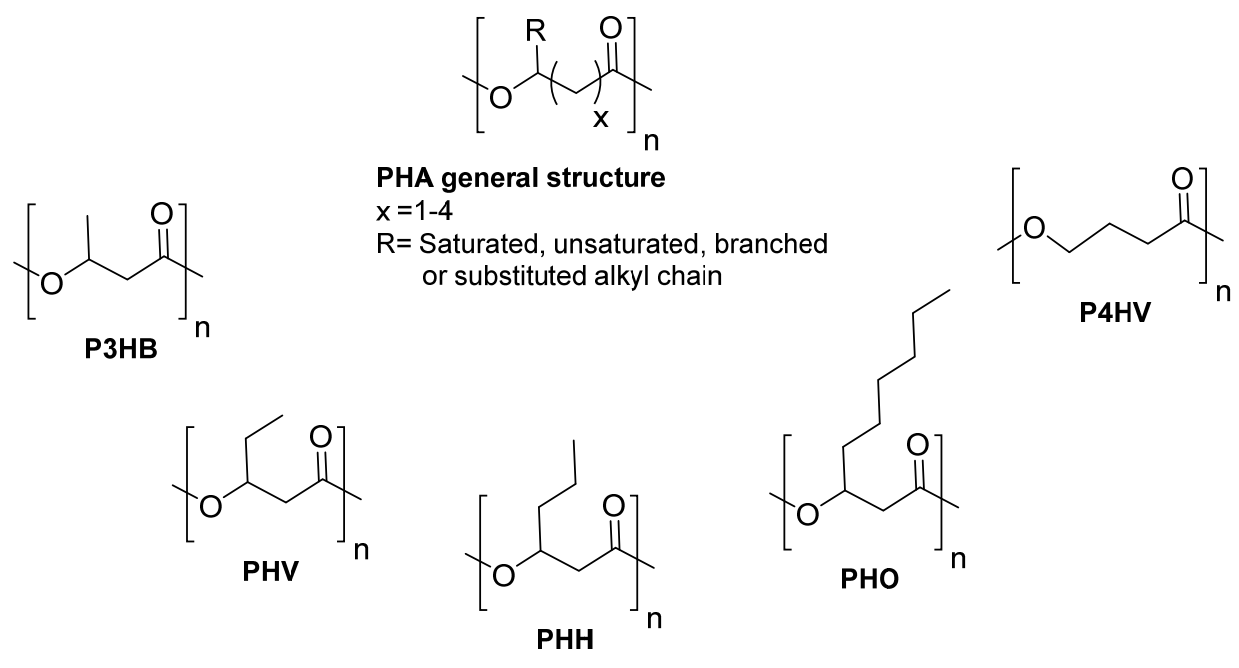

Figure 42. Synthesis and structures of main poly(hydroxyalcanoate)s, PHAs

Depending on the bacterial species and growth conditions, it is possible to produce homopolymers, random and block copolymers of PHA. As a general feature, PHAs exhibit Tgs from $-40^{\circ} \mathrm{C}$ to $4^{\circ} \mathrm{C}$ and melting temperatures in between 50 to $180^{\circ} \mathrm{C} .{ }^{205}$ Hence PHAs characteristics range from elatomers to polypropylene mimics.

Poly(3-hydroxybutyrate), P3HB, discovered in bacteria by Lemoingne in 1923, is the most widespread and best characterized PHA. Indeed, P3HB is accumulated by a wide range of Gram-positive and negative organisms under conditions of nutrient limitation. ${ }^{205} \mathrm{P} 3 \mathrm{HB}$ can be produced with final concentration over $200 \mathrm{~g} / \mathrm{L}$ with $\mathrm{PHB} \%$ over $75 \%$ and productivity over $3 \mathrm{~g} \cdot \mathrm{L}^{-1} \cdot \mathrm{h}^{-1}$. Such biologically produced $\mathrm{P} 3 \mathrm{HB}$ is a brittle semi-crystalline (elongation at break $<5 \%, \mathrm{Tm}=180^{\circ} \mathrm{C}$ ) isotactic stereoregular polyester with $100 \%$ of $\mathrm{R}$ configuration. $\mathrm{P} 3 \mathrm{HB}$ molecular weights differ with respect to the organism, conditions of growth and method of extraction and can vary from about $50 \mathrm{~kg} / \mathrm{mol}$ to over one million $\mathrm{g} / \mathrm{mol}$.. This inherent brittleness of P3HB limits its most widespread practical application, such as packaging. Two approaches have been pursued to improve P3HB mechanical properties. One approach is the microbial synthesis of copolymers containing other units such as 3-hydroxyvalerate or 4hydroxybutyrate along the polymer chain. In such a case, the physical and thermal properties of the PHAs can be regulated by varying the copolymer composition. The second approach is the blending of P3HB with other polymers, including non-biodegradable polymers (PVAc, PMMA), biodegradable polymers (PVA, PEO), bio-sourced polymers (PCL, PLA, polysaccharides). 
PHAs are completely biodegradable and compostable in different environments including marine water. They are water-insoluble and relatively resistant to hydrolytic degradation. They possess good UV resistance but poor resistance to acids and bases. They are non-toxic, biocompatible and hence suitable for medical applications, the reasons of which throve considerable commercial interest. The formulation of PHAs, with tailored additives and blends improved greatly their mechanical properties as well as their processability. These advances will improve the capacity of PHAs to penetrate markets. Nevertheless, limitations still persist such as their elevated cost $(7 € / \mathrm{kg})$. In addition, the extraction of PHAs from the culture media still represents an issue and new processes have to be developed for an efficient and economical large-scale production of PHAs.

\section{E. Bio-aromatic polymers}

The main resource for the synthesis of bio-based aromatic monomers is Lignin. Lignin represents $20-25 \%$ of wood in weight with a world production from pulp industry reaching 50 million tons/year. Nowadays, the main valorization of lignin is its conversion into energy. It is worth mentioning that such feedstock is not in competition with food industry, in contrast to some other renewable resources.

Lignin is a complex biopolymer composed of three different cinnamyl alcohols (Figure 43), named as pcoumaryl alcohol (a), coniferyl alcohol (b) and sinapyl alcohol (c), these three substrates differing by the number of methoxy groups linked to the phenol moiety.

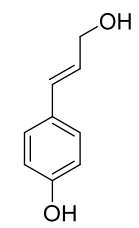

(a)

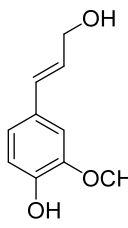

(b)

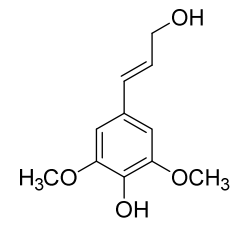

(c)

Figure 43. Monolignol monomers: (a) p-coumaryl alcohol, (b) coniferyl alcohol and (c) sinapyl alcohol

The cinnamyl alcohols ratios (also called monolignols) in lignin vary from the plant species. Accordingly, the nature of the linkages in between each monolignol species varies as well (Figure 44). The most common linkage presents in lignin is the $\beta-\mathrm{O}-4$ (aryl-ether) linkage that counts for about $50 \%$. Such linkage is usually a main target in the industrial processes aiming at the pulp delignification. The other linkages $\beta-5, \beta-1, \beta-\beta, 5-5$, and $4-\mathrm{O}-5$, are generally much more difficult to cleave.

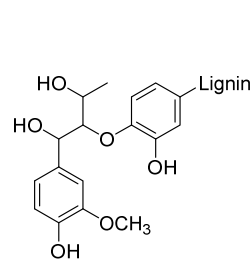

(a)

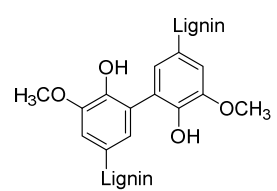

(e)

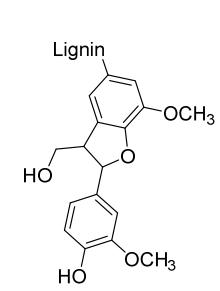

(b)

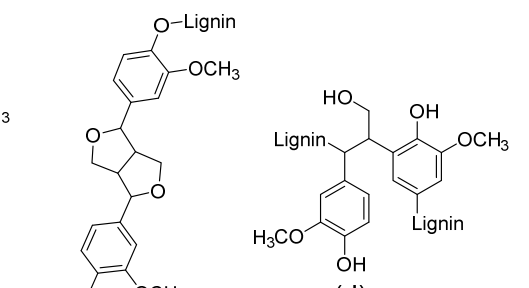

(d)

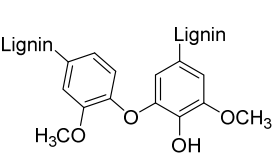

(f)

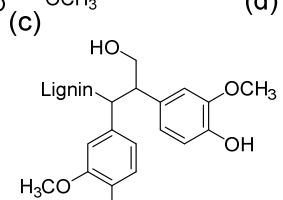

$\mathrm{OH}(\mathrm{g})$

Figure 44. Main structural units between monolignols observed in lignin, (a) $\beta-O-4$, (b) $\beta-O-5$, (c) $\beta-\beta$, (d) $\beta-1$, (e) $5-5$, (f) $4-\mathrm{O}-5$, , (g) $\beta-5$

Knowing these main features, it is worth mentioning how difficult it is to precisely determine the various lignin structures. However, a hypothetical structure of lignin is generally considered as indicated in Figure 45. 
Several processes have been industrially developed to remove lignin from the lignocellulosic biomass, which can be divided into two categories depending on the presence of sulfur in the raw material. Lignin, isolated from sulfite and kraft processes contains sulphur. The main producers are Borregaard Lignotech, Rayonier and Westvaco. Lignin, which is isolated with soda or organosolv processes, does not contain sulphur atom in its structure. The major producers are Greenvalue, CIMV and Lignol Innovations. The process of isolation induces some chemical modifications of the native lignin that strongly affects the lignin structure and thus renders its structural elucidation more difficult.

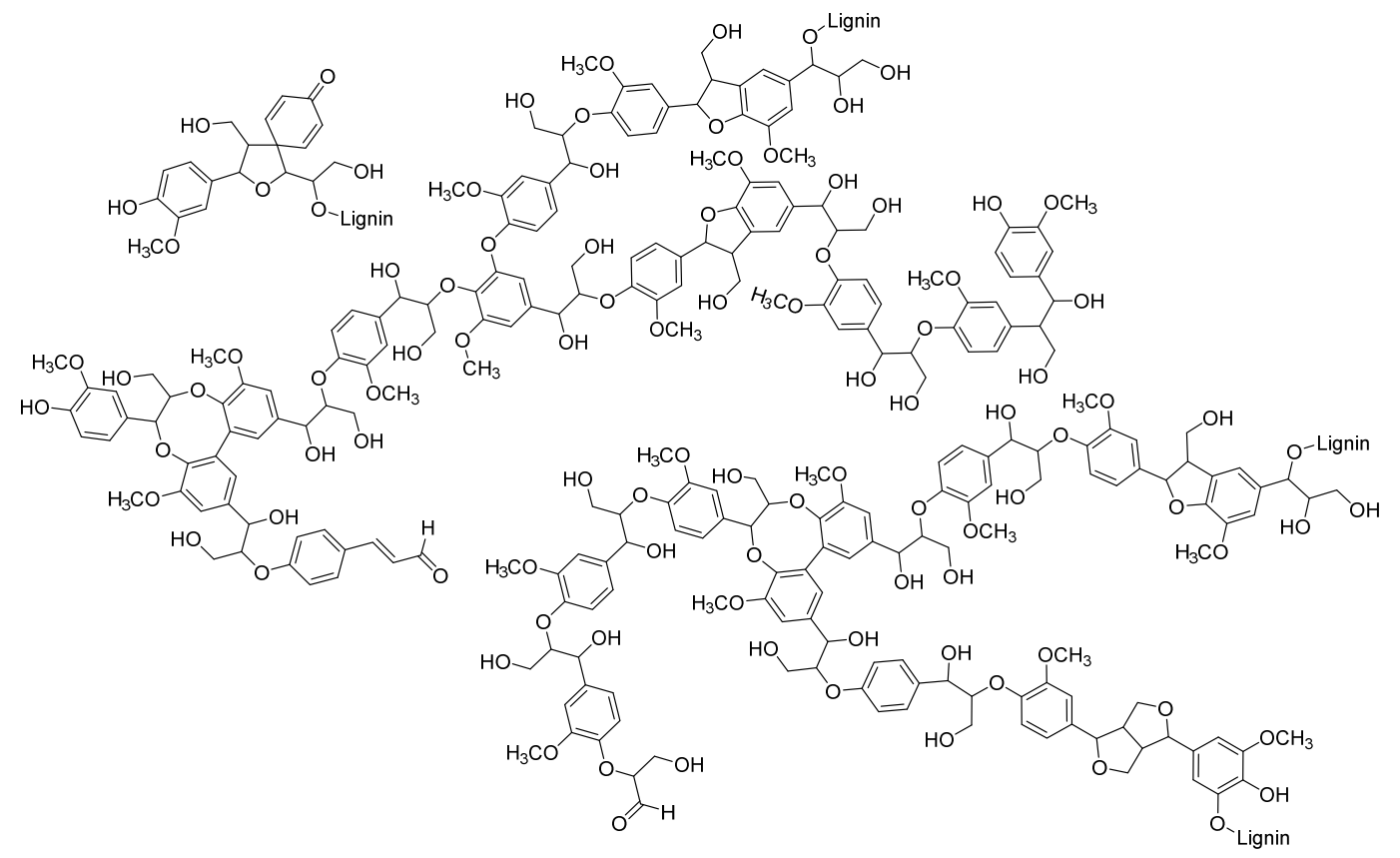

Figure 45. Hypothetical structure of Lignin

\section{Lignin as a macromononer}

A first attempt to valorize lignin as polymer precursor is to employ it as a macromonomer. ${ }^{206}$ The main reported results concern the synthesis of polymeric materials through polyaddition and polycondensation reactions. Two important issues with respect to lignin have to be mentioned. The first one concerns the processability of lignin. Indeed, lignin is obtained as a solid, with an estimated molecular weight around $10 \mathrm{~kg} / \mathrm{mol}$., and cannot be liquefied. The second issue is finally the rather low reactivity of lignin due to some steric hindrance.

\section{i. Polyurethanes}

Thanks to the presence of phenolic and aliphatic hydroxyl groups, lignin was used as a polyol for the synthesis of polyurethanes. Due to its rigid structure, the lignin-based polyurethanes are stiff materials. However, to compensate its poor processability, lignin was reacted with soft aliphatic diisocyanates. Similarly and in order to enhance the reactivity of lignin as a polyol and to ease its processability, some studies reported its derivatization with poly(ethylene glycol) or poly(propylene glycol). ${ }^{206-208}$ In such a case, the lignin structure is diluted within the final polyurethane network. As a main target of the PU industry, the synthesis of flexible and rigid foams from lignin-based polyol is a challenge that was reported but still requires strong improvements. ${ }^{209}$ 


\section{ii. Polyepoxide networks}

Polyepoxide networks are an important class of materials used in coatings, adhesives, composites and electronic materials. The direct use of lignin as macromolecular structure in polyepoxide networks was limited for a long time to the use in blends and as ring-opening reagents. ${ }^{210-213} \mathrm{In}$ other routes, lignin hydroxyl functions were converted to acidic ones via esterification with succinic anhydride, increasing the reactivity towards epoxides. ${ }^{214}$ The interest of using lignin as epoxide substrate increased and various approaches with lignin-based epoxy thermosetting polymers were reported. A main approach consists in derivatizing lignin with epichlrohydrin to graft epoxy moiety onto lignin structure (Figure 46). ${ }^{215}$ Generally the epoxidized lignin is mixed with other polyepoxides and then cured with various hardeners. The authors observed that the higher the lignin content, the higher the cross-linking density, resulting in a higher glass transition temperature, lower swelling ratio and increased stiffness in comparison to a reference network not embedding lignin structure.
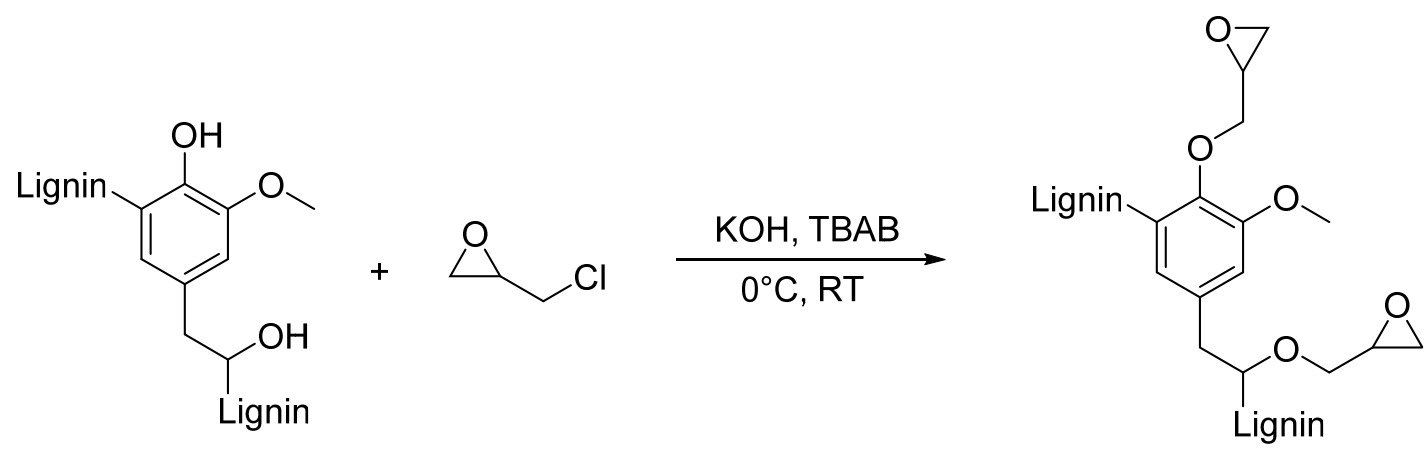

Figure 46. Glycidylation of organo-solv lignin with epichlorohydrin

\section{iii. Phenolic thermosets}

Phenolic thermosets represent an important class of polymeric materials (adhesives) mainly based on two monomers, i.e. phenol and formaldehyde. Both monomers, notably formaldehyde, should be replaced because of health and environmental concerns. Indeed, lignin appeared as a good candidate as phenol precursor. Some studies reported the incorporation of lignin in phenol-formaldehyde thermosets. ${ }^{216,217}$ Again, only a fraction of lignin was used in addition to phenol due to the lack of processability of lignin and to its uncomparable lower reactivity with respect to phenol. As a general trend, the higher the lignin content in the thermoset, the higher the thermal stability.

With respect to this polymer family, one important challenge remains the increase of phenolic functions on the lignin substrate. With this aim, the demethylation of methoxy groups from monolignol units appeared to be an efficient strategy to consider. ${ }^{218-220}$

\section{Depolymerization of lignin}

As already discussed, lignin is the major source of aromatic compounds from biomass. A big challenge is thus to selectively and efficiently depolymerize lignin into well-defined, processable and low molecular weight aromatics. Amongst such substrates, vanillin and ferulic acid appeared to be substrates of interest for the further synthesis of (semi)aromatic polymers (Figure 47). 


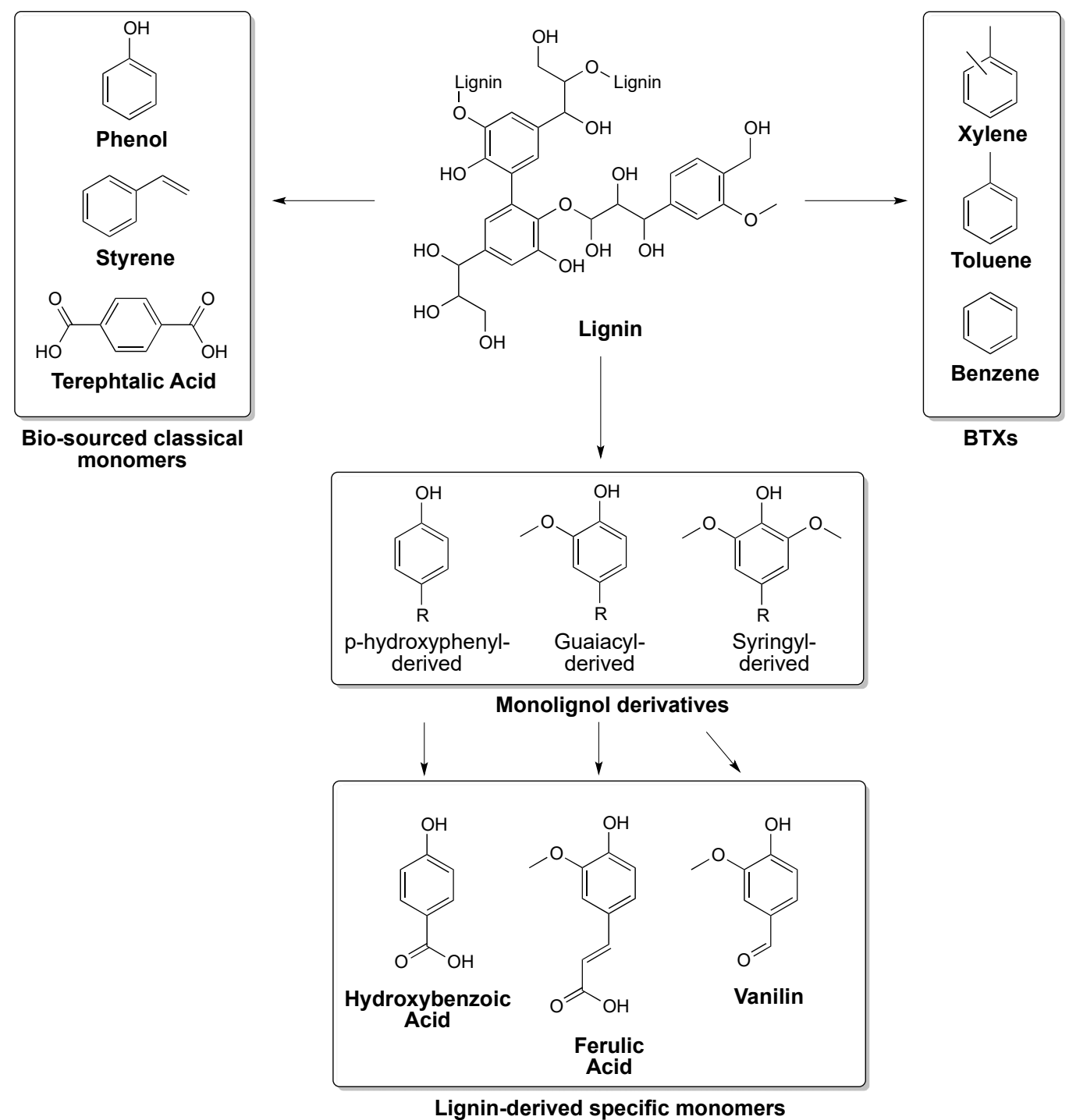

Figure 47. Targeted molecules obtained by depolymerization of lignin under oxidative or reductive conditions

\section{i. Reductive cleavage of lignin}

The depolymerization of lignin can be performed through reductive or oxidative catalyses. In the reductive process, lignin is treated at high temperature under pressurized $\mathrm{H}_{2}$ in the presence of transition metal catalysts. This process is a two-step-procedure in which the first step is the ether bond ( $\beta-\mathrm{O}-4, \alpha-\mathrm{O}-$ 4) cleavage and the second step, a hydrodeoxygenation of the formed molecules. The main transition metal catalysts used for the reductive depolymerization of lignin are platinum, ruthenium, rhodium, and palladium. ${ }^{221,222}$ A lot of studies focused on the reductive depolymerization of lignin models for the production of benzene, toluene and xylene (BTX products), Figure 48. To our knowledge, this method is not mature enough to be effective on crude lignin and required some improvements in terms of catalytic efficiencies and processes.<smiles>[R]c1ccc(Oc2ccccc2)cc1</smiles>

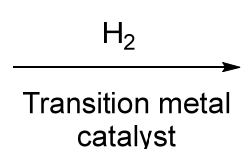<smiles>[R]c1ccccc1</smiles><smiles>[R]c1ccc(O)cc1</smiles>

Figure 48. Model reaction of reductive cleavage of bisaromatic compounds towards the formation of BTXs 
With respect to polymer synthesis, a big challenge, is obviously the access through this reductive process to xylene, intermediate used for the synthesis of terephthalic acid and thus bio-poly(ethylene terephtalate), bio-PET, as already represented in Figure 5.

\section{ii. Oxidative cleavage of lignin}

Another strategy is the lignin depolymerization under oxidative conditions. Contrary to the reductive route, the oxidative cleavages of $\mathrm{C}-\mathrm{C}$ and $\mathrm{C}-\mathrm{O}$ bonds lead to high value functional molecules, such as ferulic acid or vanillin (Figure 47). ${ }^{223}$ Interestingly, ferulic acid exhibits anti-oxidant, anti-ageing, antidiabetic and anticancer properties while vanillin is widely used as aroma in agro-industry.

Since twenty years, a huge variety of oxidative process, oxidants and catalysts were tested. As for the reductive route, the main studies were reported on model molecules and not directly on lignin.

The main catalysts reported for the oxidative cleavage of lignin are organo-metalic complexes. ${ }^{23,224}$ Polyoxometalates (POM) have also been studied as oxidative catalysts for lignin depolymerization. These metal-oxygen clusters are considered as green catalysts. ${ }^{225}$ Zhao et al. performed oxidations of lignin under pressurized oxygen in a methanol/water mixture in the presence of POM, that lead to the production of acids with yields ranging from 12 to $65 \% .{ }^{226}$ Following a biomimetic approach, metalloporphyrin catalysts were also employed as analogues of peroxidases, enzymes produced by fungi capable of depolymerizing lignin. The latter catalysts are less expensive and more versatile than their enzymatic analogues. ${ }^{227}$ Another method which employs a TEMPO derivative in a formic acid aqueous solution containing sodium formate and allowing the oxidation of lignin at $110^{\circ} \mathrm{C}$ during $24 \mathrm{~h}$ was also reported. Low molecular weight aromatic compounds were obtained in good yields $(60 \%)$ through this process. ${ }^{228}$

Nowadays, Borregaard is the only industry producing functionalized aromatic molecules from lignin and in particular vanillin. Thanks to an oxidative process performed on lignosulfonates in the presence of copper sulfate in alkaline conditions and under pressurized oxygen, 1500 tons of vanillin are produced each year with $7 \%$ yield.

\section{Lignin-based thermosets and thermoplastics}

Most of the polymers produced from lignin derivatives deal with vanillin, ferulic acid or guaiacol compounds. Due to the high amount of available literature in the field, it is impossible to perform a thorough review of all the results. It is however worth mentioning the review of Cramail and coll. on the wide scope of monomers and polymers which can originate from lignin-derivatives. ${ }^{229}$ It is encouraged to refer to this work, should complementary information be needed.

\section{i. Vinyl ester and cyano ester thermosets}

Vinyl ester resins are thermoset polymers containing acrylate or methacrylate functions. The latter are used in coatings and composites materials. Wool et al. reported in 2012 a fully vinyl ester thermoset obtained from methacrylated vanillin derived monomer. ${ }^{230}$

Cyanate esters are aromatic molecules in which the phenol group is replaced by a cyanide (NCO) function. In the curing process, the trimerization of the cyanide group into triazine occurs either at high temperature or at low temperature in the presence of metal catalysts. Two studies reported the synthesis of cyanate ester thermosets from vanillin. ${ }^{231}$ The so-formed vanillin-based thermosets exhibited similar properties than commercial cyanate ester thermosets. 


\section{ii. Epoxy thermosets}

As already discussed, the main molecule employed for the synthesis of industrial epoxy thermoset is the bisphenol-A, BPA. However, due to the toxicity of this compound, a lot of studies reported in this field aim at preparing bio-based epoxy thermosets avoiding the use of BPA. Caillol and co-workers described the synthesis of epoxy thermosets from bisepoxide of vanillin, vanillyl alcohol and vanillic acid. ${ }^{232-234}$ These two last components are commercial compounds and can be easily obtained by reduction or oxidation of vanillin. These bisepoxides obtained by glycidylation reaction with epichlorohydrin could react with isophorone diamine to produce epoxy thermosets (Figure 49).

In order to mimic the DGEBA-based resins, the authors have also synthesized bisepoxide oligomers of vanillin (Figure 49). By playing on the bisepoxide oligomer chain length, the cross-linking of the polyepoxide network could be adjusted to target particular Tg.
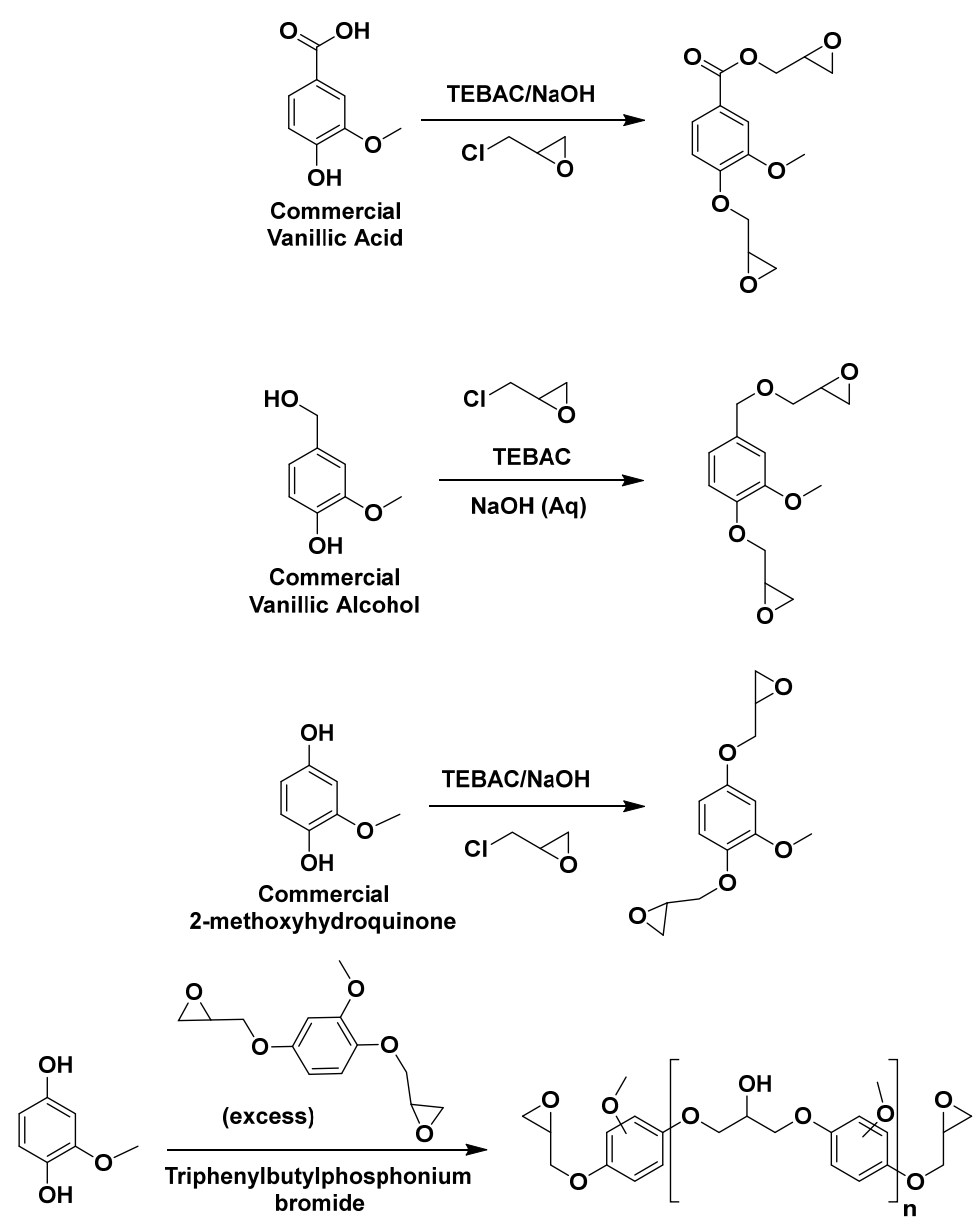

Figure 49. Synthesis of bisepoxides by chemical modification of vanillin

\section{iii. Polyesters}

\section{Direct polymerization methods}

The first example of polymerization of vanillic acid was described in the 50s. Indeed, vanillic acid was converted to carboxylate by etherifying the phenolic moiety with ethylene dihalides (Figure 50, a). Subsequently, the carboxylate was esterified with ethylene glycol and condensed to linear semi-cristalline polyester exhibiting $\mathrm{Tg}$ of $80^{\circ} \mathrm{C}$ and $\mathrm{Tm}$ of $210^{\circ} \mathrm{C} .{ }^{235}$ Later, in the $80 \mathrm{~s}$, other strategies were developed 
to synthesize vanillic and syringic acid-based polymers. ${ }^{236}$ In a second synthetic pathway, the phenolic moiety of vanillic acid was reacted with ethylene oxide, yielding an $\mathrm{AB}$ synthon able to selfpolycondensate (Figure 50, b). Viscometry molecular weights indicate that the first synthetic pathway (a) provides polyesters exhibiting higher molecular weight, around $50000 \mathrm{~g} / \mathrm{mol}$ both for vanillic acid and syringic acid in comparison to polyesters produced from the second method (b), $30000 \mathrm{~g} / \mathrm{mol}$ and $12000 \mathrm{~g} / \mathrm{mol}$, respectively. The polyesters prepared through pathway (a) showed $\mathrm{Tg}$ of $69^{\circ} \mathrm{C}$ and $\mathrm{Tm}$ of $212^{\circ} \mathrm{C}$ in the case of vanillic acid and $\mathrm{Tg}$ of $58^{\circ} \mathrm{C}$ and $\mathrm{Tm}$ of $172^{\circ} \mathrm{C}$ for syringic acid. From the second method (b), the so-formed polyesters showed $\mathrm{Tg}$ of $55^{\circ} \mathrm{C}$ and $\mathrm{Tm}$ of $254{ }^{\circ} \mathrm{C}$ in the case of vanillic acid and $\mathrm{Tg}$ of $45^{\circ} \mathrm{C}$ and $\mathrm{Tm}$ of $73{ }^{\circ} \mathrm{C}$ for syringic acid. Interestingly, the polymer produced from vanillic acid exhibits thermal properties very close to PET's $\left(\mathrm{Tm}=265^{\circ} \mathrm{C}, \mathrm{Tg}=67^{\circ} \mathrm{C}\right)$.

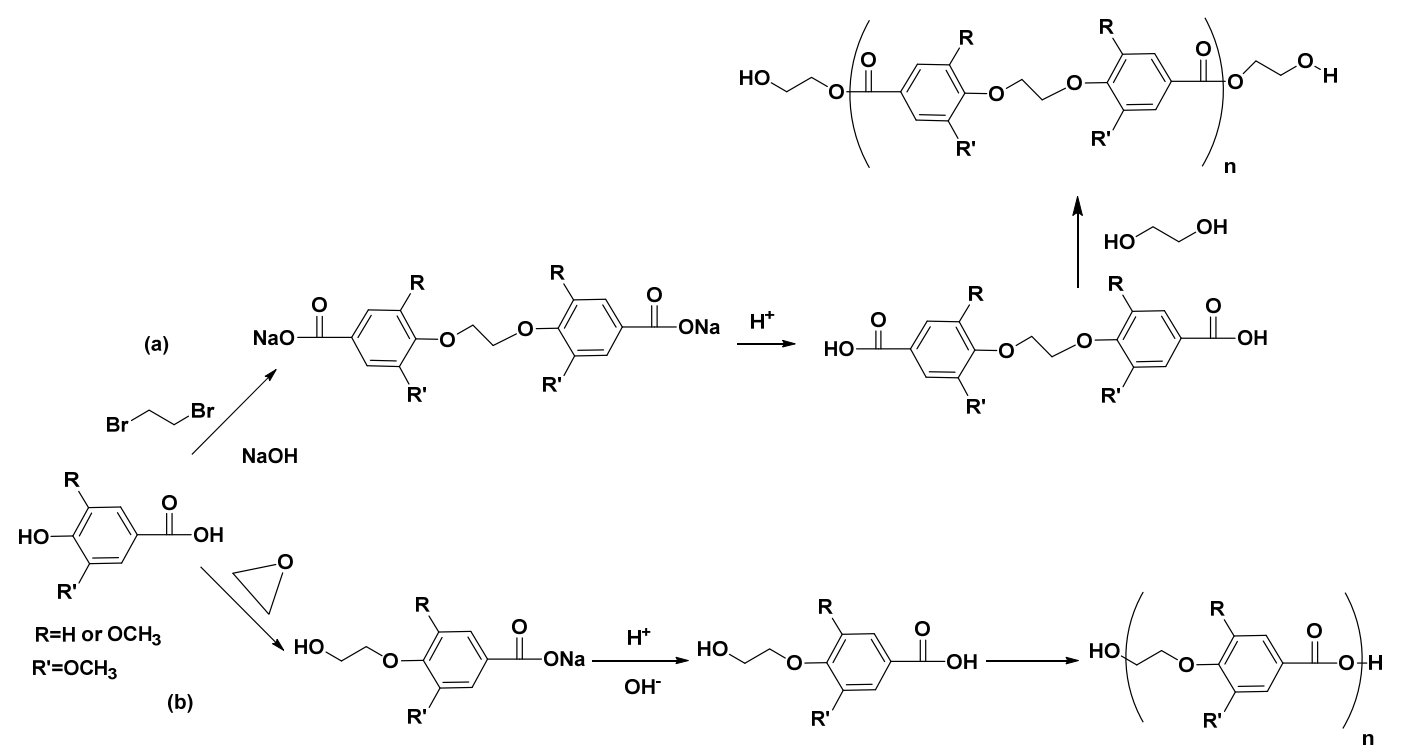

Figure 50. Polyesters from vanillic acid $(\mathrm{R}=\mathrm{H})$ and syringic acid $\left(\mathrm{R}=\mathrm{OCH}_{3}\right)$

As fas as vanillic acid is concerned, a lot of studies were reported on thermotropic polyesters. Thermotropic polymers exhibit liquid crystal behavior in their molten form. The thermotropic polyesters described in the literature exhibit high mechanical strength, chemical resistance at elevated temperature and good flame retardancy due to the presence of aromatic rings and high order in their structures. Poly(vanillic acid) was firstly synthesized by Kricheldorf and co-workers from two different routes. ${ }^{237}$ Based on these studies, various other polymers were synthesized by copolymerization of vanillic acid with different comonomers. Vanillic acid compared to other monomers like terephtalic acid or bisphenolA brings to the terpolymers, faster polycondensation rate, higher molecular weights and lower melting temperatures. These vanillic acid-based thermotropic polymers attracted attention for biomedical applications thanks to their good thermomechanical properties and processability. Indeed, the synthesis of bioresorbable polyesters from vanillic acid and p-hydroxybenzoic acid was recently reported. ${ }^{238}$

In the current context of "green" chemistry, $100 \%$ biobased polyesters were synthesized from vanillin, ferulic acid and vegetable oil derivatives. Meier and coll. produced polyesters from ferulic acid by polycondensation (Figure 51). ${ }^{239}$ The carboxylic moiety of ferulic acid was first esterified with methanol and the resulting hydrogenated methyl ferulate was then reacted with 2 equivalents of ethyl carbonate, yielding an $\mathrm{AB}$ monomer, which was polymerized in the presence of TBD as a catalyst; the polymer soformed exhibits a molecular weight of $5400 \mathrm{~g} / \mathrm{mol}$ and a semi-crystalline feature with a $\mathrm{Tg}$ of $-27^{\circ} \mathrm{C}$ and a Tm of $25^{\circ} \mathrm{C}$. Amorphous polyesters were also synthesized by copolymerization with fatty methylester derivatives. 
The polycondensation of vanillin and fatty acid derivatives was also investigated (Figure 51). ${ }^{240}$ The aldehyde moiety of vanillin was converted into alcohol and etherification of the phenol moiety was performed with an undecenoic acid derivative. The thiol-ene addition of methylthioglycolate on the unsaturation leads to an $\mathrm{AB}$ monomer able to undergo homopolymerization. The polymer so-formed is semi-crystalline with a $\mathrm{Tg}$ of $-13^{\circ} \mathrm{C}$ and a Tm of $77^{\circ} \mathrm{C}$. Polymers with a higher crystallinity were also synthesized by copolymerization with fatty acid derived esters.
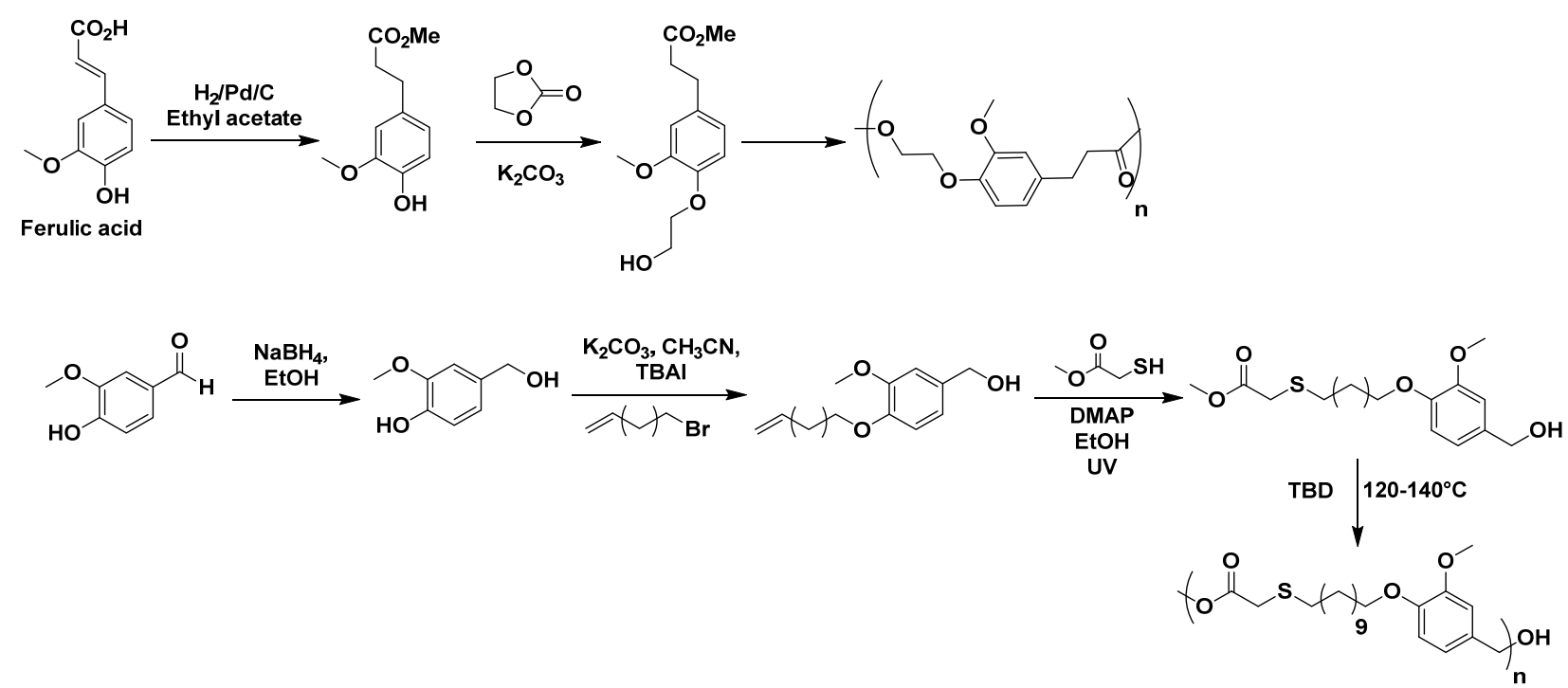

Figure 51. Synthesis of ferulic acid- and vanillin-based polyesters by polycondensation in a sustainable way.

Miller and coworkers reported the synthesis of bio-renewable poly(ethylene terephthalate) mimics, derived from lignin-derived substrates (Figure 52). ${ }^{241}$ The reaction of vanillin and acetic anhydride leads to both Perkin reaction and acetylation of the phenolic group. The resulting compound was hydrogenated and the generated acetyldihydroferulic acid was homopolymerized. $\mathrm{ZnAc}_{2}$ proved to be the most efficient catalyst. The polyester so-formed exhibits a molecular weight of $17800 \mathrm{~g} / \mathrm{mol}, \mathrm{Tm}$ of $234^{\circ} \mathrm{C}, \mathrm{Tg}$ of $73{ }^{\circ} \mathrm{C}$ and a $50 \%$ thermal decomposition temperature $\left(\mathrm{Td}_{50 \%}\right)$ at $462{ }^{\circ} \mathrm{C}$. Such values are similar to the corresponding values of PET $\left(\mathrm{Tm}=265^{\circ} \mathrm{C}, \mathrm{Tg}=67^{\circ} \mathrm{C}, \mathrm{Td}_{50 \%}=470{ }^{\circ} \mathrm{C}\right)$.

The same group also synthesized poly(alkylenehydroxybenzoate)s (PAHBs) from vanillin, 4hydroxybenzoic acid and syringic acid (Figure 52) in order to target semi-aromatic polyesters with a wide range of thermo-mechanical properties. ${ }^{242}$ These aromatic aldehydes were oxidized into corresponding carboxylic acids and the phenol moiety was derivatized with 2-chloroethanol or 3-chloropropan-1-ol. The resulting hydroxy acid monomers $(\mathrm{AB})$, were homopolymerized, in the presence of antimony oxide as catalyst. The thermo-mechanical properties of the so-formed polyesters are varying with respect to the aromatic unit substitution. Indeed, the substitution on the aromatic ring increases the free volume of the polymer chains, which decreases the Tg. In addition, the length of the aliphatic segment between the aromatic units has also an impact on the thermal properties. In this series of polymers, the glass transition temperature was tuned between 50 and $70{ }^{\circ} \mathrm{C}$ and the melting temperature between 170 and $239{ }^{\circ} \mathrm{C}$. 

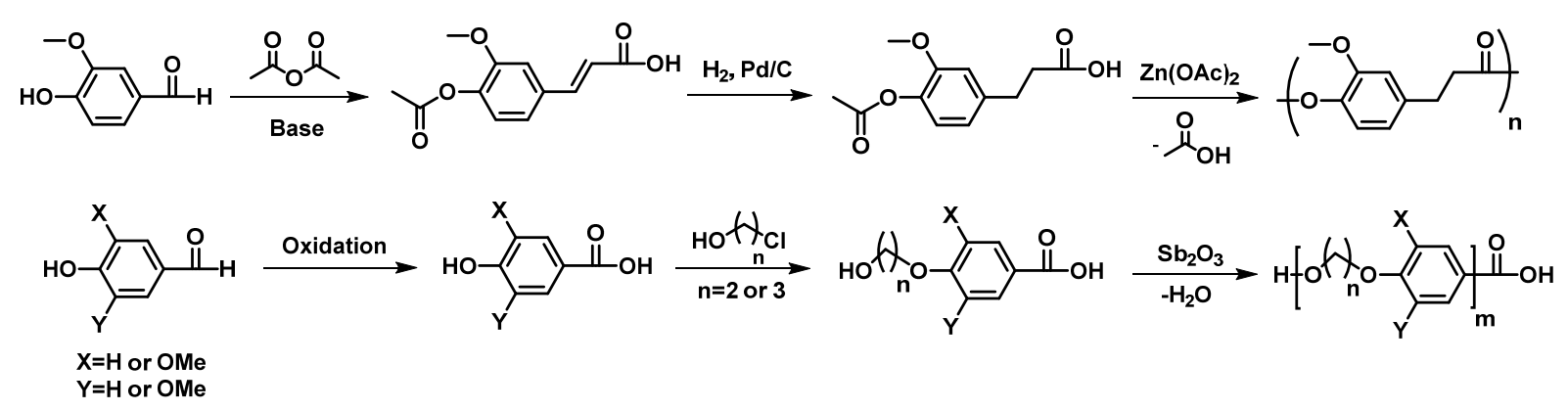

Figure 52. Synthesis of poly(alkylenehydroxybenzoate)s from lignin derivatives

\section{Synthesis and polymerization of symmetrical difunctional monomers}

An alternative access toward difunctional monomer from vanillin or lignin derivatives is based on the coupling of phenolic substrates. This coupling could occur either on the phenol yielding dicarbonyl, or on the carbonyl yielding bisphenol. ${ }^{243-245}$ These building blocks were further used for polyester synthesis.

In order to produce difunctional symmetrical monomers, Grelier and coll. have investigated the enzymatic coupling, with the help of laccase from Trametes Versicolor, of several phenolic compounds potentially derived from lignin (Figure 53). ${ }^{246}$
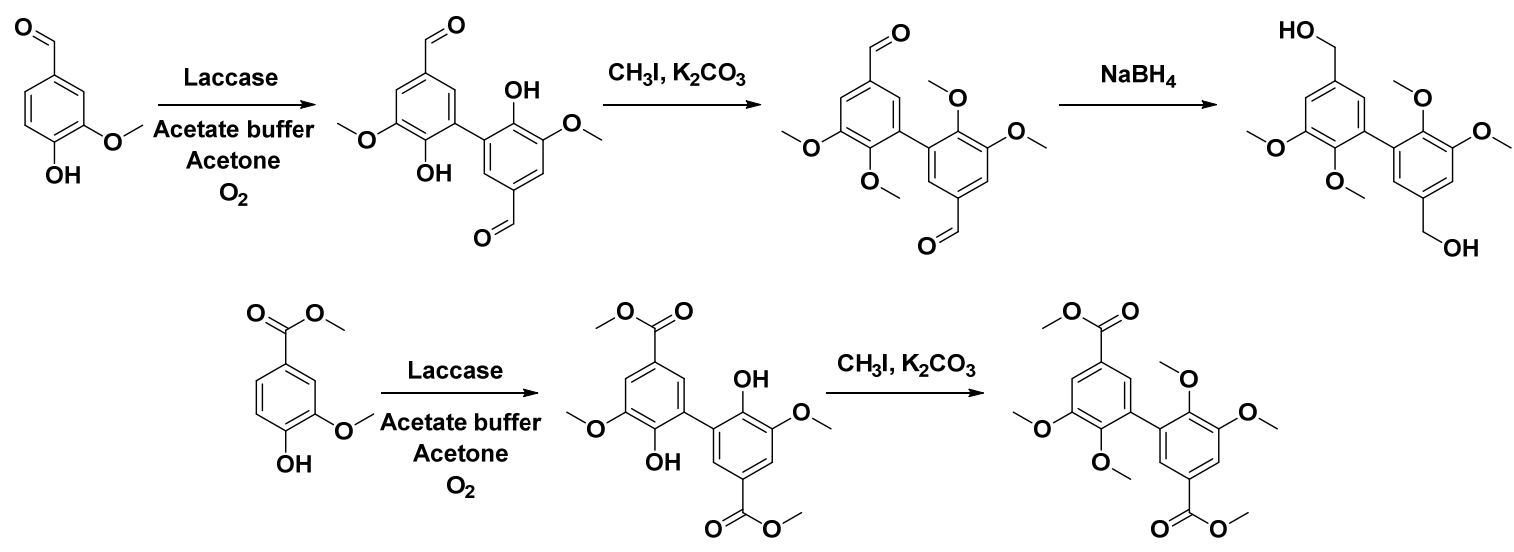

Figure 53. Synthesis of methylated divanillyl diol and methylated dimethyl divanillate from vanillin

The chemical modification of these building blocks enabled the synthesis of symmetrical monomers for the design of renewable (semi)aromatic polyesters (Figure 54). ${ }^{247}$ The methylation of the phenol prevents the phenol moiety to be involved in the polycondensation. The reactivity of methylated divanillyl diol and methylated dimethyl divanillate towards polycondensation was investigated on the copolymerization with dimethyl sebacate and 1,10-decanediol. Polyester molecular weights of $65000 \mathrm{~g} / \mathrm{mol}$ were reached for methylated divanillyl diol and of $20000 \mathrm{~g} / \mathrm{mol}$ for methylated dimethyl divanillate. Considering this difference in reactivity, a series of polyesters were synthesized by transesterification of methylated divanillyl diol with several diesters of aromatic, aliphatic or cyclo-aliphatic structures (Figure 54). The polyesters displayed a $\mathrm{Tg}$ ranging from -5 to $139^{\circ} \mathrm{C}$, influenced by the structure of the co-monomer and 5 $\mathrm{wt} \%$ loss temperature above $300{ }^{\circ} \mathrm{C}$. 

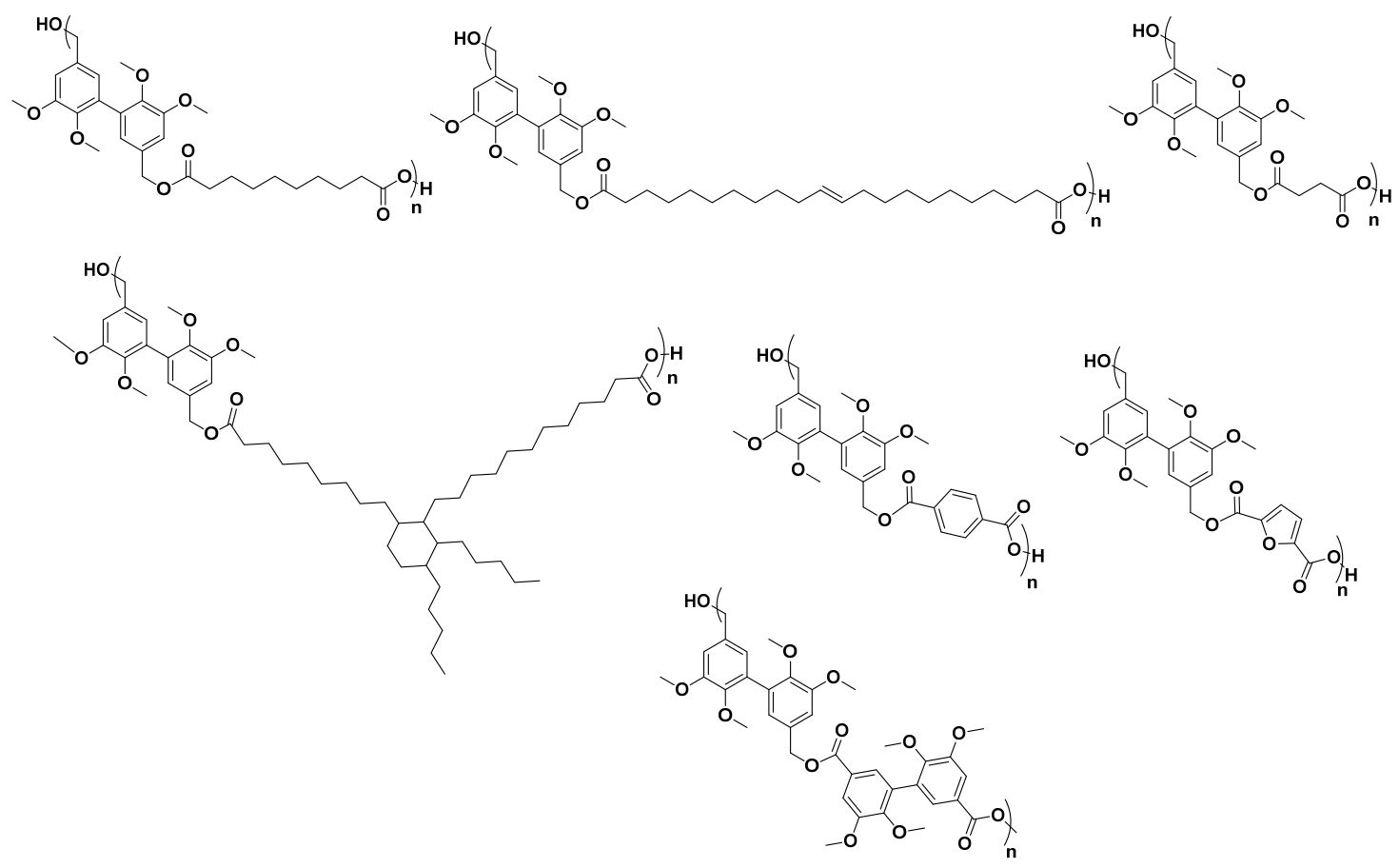

Figure 54. Structures of polyesters synthesized from methylated divanillyl diol and various diesters

\section{Synthesis and polymerization of bis-unsaturated esters}

Other polymerization techniques were employed to produce polyesters from lignin derivatives (Figure 55). Notably, Meier and coll. prepared bis-unsaturated esters from ferulic acid and vanillin, which were polymerized via ADMET or thiol-ene methodologies. ${ }^{240,248}$ The polymers synthesized via thiol-ene polyaddition were found semi-crystalline whereas the ones synthesized by ADMET were amorphous.

Allais and coll. also modified bisphenols derived from ferulic acid to obtained bis-unsaturated esters by etherification with various bromo-alkenes in order to perform ADMET polymerization. ${ }^{249}$ Amorphous polymers were obtained with low Tgs, from -21.6 to $18.2^{\circ} \mathrm{C}$ and $5 \mathrm{wt} . \%$ loss temperatures between 291 and $333{ }^{\circ} \mathrm{C}$. Our group also reported the synthesis of a bisunsaturated diester and its polymerization by ADMET methodology. ${ }^{250}$ The latter was obtained from methylated dimethylvanillate dimer and undecenol. ADMET polymerization was performed in Polarclean solvent which was selected for its high boiling point, its compatibility with Grubbs catalyst and its sustainability. ${ }^{251}$ 

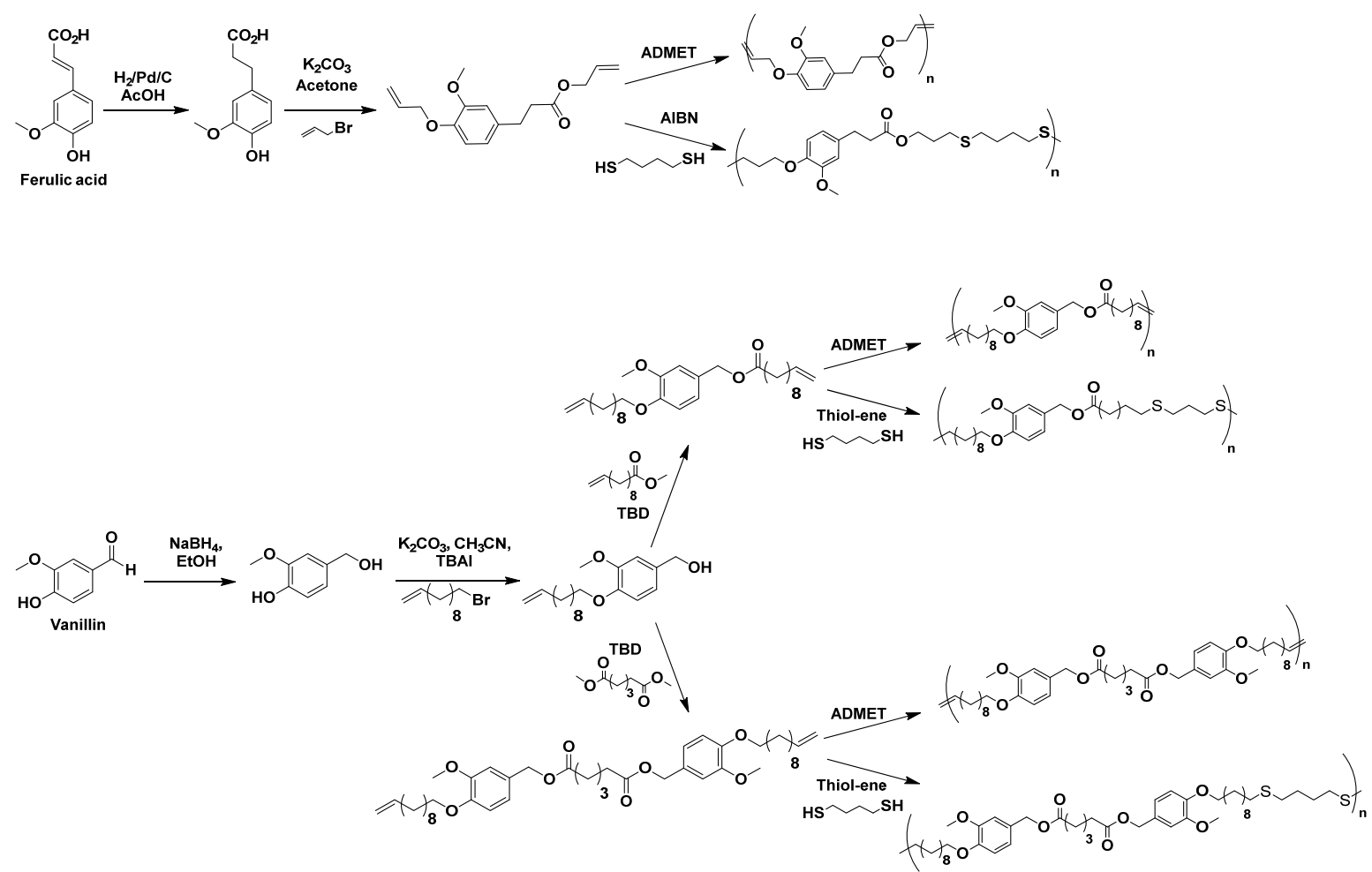

Figure 55. Synthesis of ferulic acid- and vanillin-based polyesters by ADMET polymerization and thiol-ene addition

\section{iv. Polyacetals}

Dialdehydes are also important monomers for the synthesis of polyacetals, a class of biodegradable polymers as acetal linkages are sensitive to hydrolysis. Miller and coll. synthesized cyclic and polycyclic polyacetal ethers from 4-hydroxybenzaldehyde, vanillin and syringaldehyde (Figure 56). ${ }^{252}$ The dialdehydes were prepared by reaction of lignin derivatives with 1,2-dibromoethane using sodium hydroxide and potassium iodide in water. Polycondensation of the dialdehydes with tetraol yields cyclic polyacetal ethers in the case of di-trimethylolpropane and spirocyclic acetal in the case of pentaerythritol (Figure 56). Molecular weights ranging between 10600 and $22200 \mathrm{~g} / \mathrm{mol}$ were reached. Polymers from 4-hydroxybenzaldehyde are semi-crystalline, whereas polymers synthesized from vanillin and syringaldehyde are amorphous due to the presence of methoxy groups. Spirocyclic polyacetals exhibit higher $\mathrm{Tg}$ than the cyclic equivalent ones (for vanillin: $129^{\circ} \mathrm{C}$ versus $80^{\circ} \mathrm{C}$ ). As already reported, syringaldehyde-based polymers show higher glass transition than vanillin-based ones $\left(152{ }^{\circ} \mathrm{C}\right.$ for the spirocyclic one against $98{ }^{\circ} \mathrm{C}$ for the cyclic equivalent). All the polymers present high $5 \mathrm{wt} \%$ degradation temperature, between 307 and $349^{\circ} \mathrm{C}$.

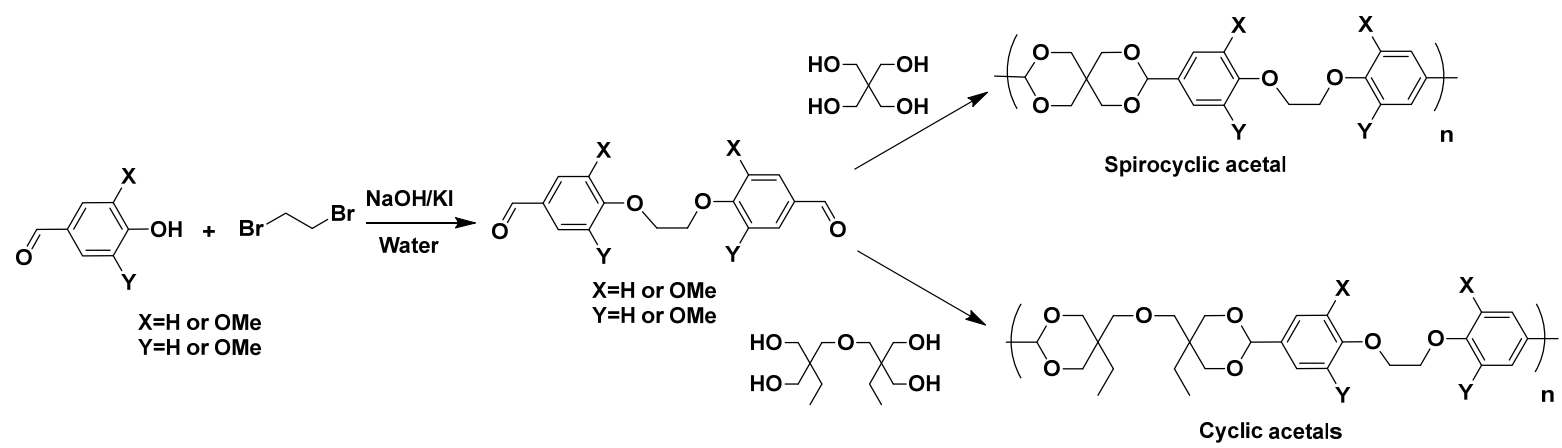

Figure 56. Synthetic pathway of polyacetals from lignin derivatives 


\section{v. Polycarbonates}

Polycarbonates are a class of materials between the commodity and the engineering plastics. The latter are synthesized by transesterification of bisphenols and phosgene or carbonates. Harvey and coll. synthesized polycarbonates by transesterification reaction between bisphenol from vanillin and diphenylcarbonate but low molecular weights were obtained. ${ }^{253}$ Biocompatible and biodegradable poly(carbonate-amide)s were also synthesized from ferulic acid and tyrosine..$^{254}$

\section{vi. Radical Polymerization}

Lignin derivatives were also modified into acrylamide and acrylate derivatives and polymerized by radical polymerization. Thermoplastic polymers with lignin derivatives as side chain groups were obtained (Figure 57). Roger and coworkers synthesized the acrylamide derivative from guaiacol and vanillin. $^{255}$ The first pathway involves the Friedel-Crafts alkylation reaction of Nhydroxymethylacrylamide with guaiacol. The second approach is based on vanillin and requires a threestep reaction: the oxime is prepared, reduced and the resulting amine reacts with acryloyl chloride. The obtained acrylamide was polymerized by free radical polymerization employing AIBN as an initiator. Despite a screening of the conditions, polymers with relatively low molecular weights, below $11000 \mathrm{~g} / \mathrm{mol}$ were obtained; branched structures and broad molar mass distributions were observed at high conversion. These observations can be attributed to the phenoxy radicals known to inhibit radical polymerization by acting as scavenger.

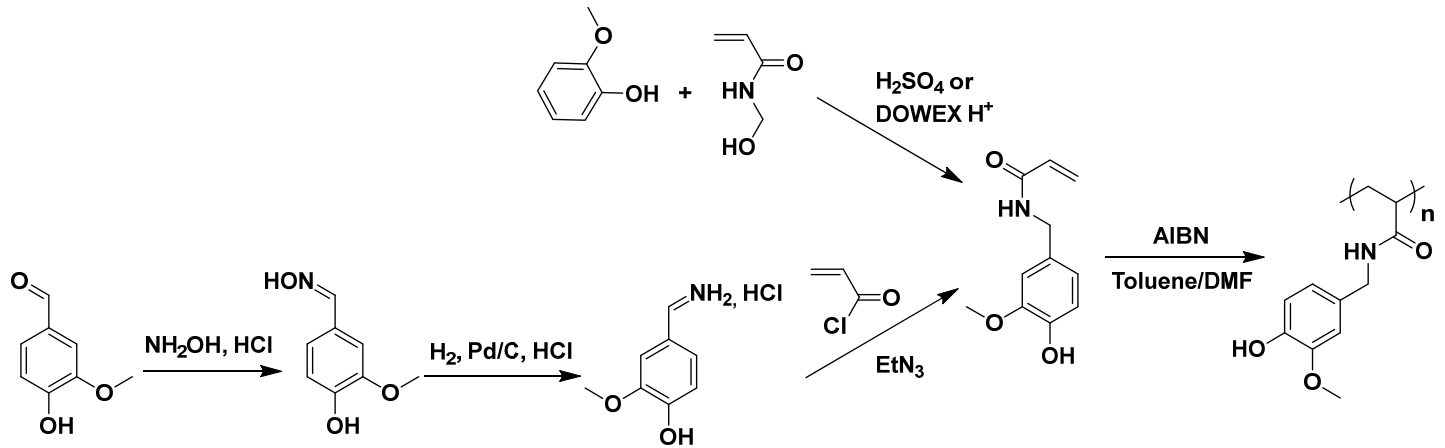

Figure 57. Synthesis of thermoplastic polymers by radical polymerization of lignin derived acrylamides

Controlled radical polymerization was also used to synthesize homopolymer and block copolymers from vanillin-derived acrylate. ${ }^{256}$ An acrylate derivative was synthesized from vanillin by esterification of the phenolic group with methacrylic anhydride (Figure 58). The latter was polymerized by reversible addition-fragmentation chain transfer (RAFT) yielding a polymer with a molecular weight of $17000 \mathrm{~g} / \mathrm{mol}$ and a dispersity of 1.34 . The latter polymer was employed as macro-chain transfer agent (CTA) for the polymerization of lauryl methacrylate, a fatty acid derivative, yielding a block copolymer with a molecular weight of $56000 \mathrm{~g} / \mathrm{mol}$ and a dispersity of 1.50 . The vanillin homopolymer exhibits a $\mathrm{Tg}$ of $120^{\circ} \mathrm{C}$ and a degradation temperature over $300{ }^{\circ} \mathrm{C}$, similar to the values of polystyrene. The block copolymer chains self-assemble in body-entered-cubic nanospheres. Other morphologies could be reached by increasing the volume fraction of vanillin.
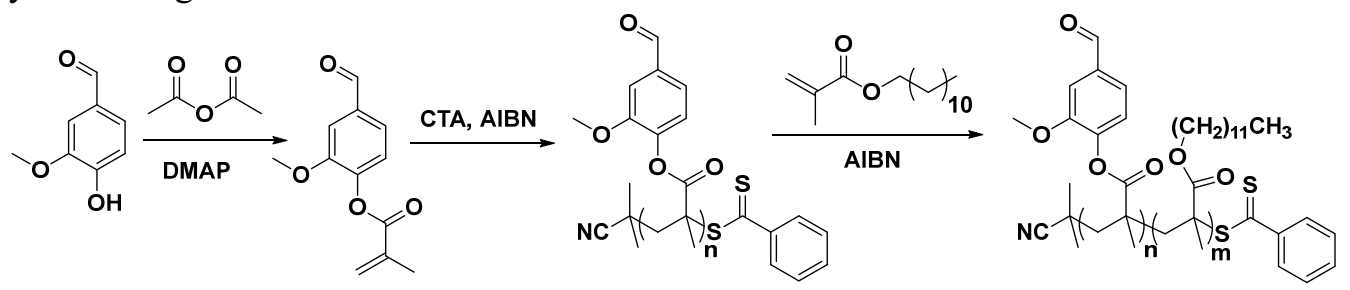
Figure 58. Synthesis of thermoplastic polymers by radical polymerization of lignin derived acrylates

\section{Polymers from vegetable oils}

\section{A. Introduction}

Vegetable oils represent one of the most promising bio-resources for the synthesis of bio-based polymers. This biomass has gained attention in the past few years as the oil structures enable chemical transformation and access to new and innovative properties for some applications. Indeed, vegetable oils are a combination, via ester bonds, between glycerol and three fatty acids with variable carbon contents and functionalities depending on the plant species and the growing conditions (Figure 59). ${ }^{257}$ Therefore, the nature of fatty acids present on triglycerides differentiates one vegetable oil from another. Still, one of the problematic encountered by vegetable oil-based polymers is the variable composition in triglycerides within a same kind of crop, preventing the access to materials with reproducible properties.

On the one hand, refined oil containing triglycerides can be valorized as monomers for thermosets or hyperbranched polymers, taking advantage of their plurifunctionality and their inherent biodegradability. Triglycerides can be polymerized as produced thanks to the naturally occurring functional groups present on fatty acids (esters, alcohol, insaturations, epoxide moieties) or after chemical modifications. On the other hand, glycerol and fatty acids can be extracted from the triglycerides and chemically transformed in several intermediates to prepare hyperbranched or linear thermoplastic polymers. The use of purified fatty acid derivatives enables the preparation of well-defined thermoplastic polymers with controlled structureproperties relationship. The different strategies adopted to prepare vegetable oil-based polymers will be detailed focusing mainly on renewable thermoplastic polymers.

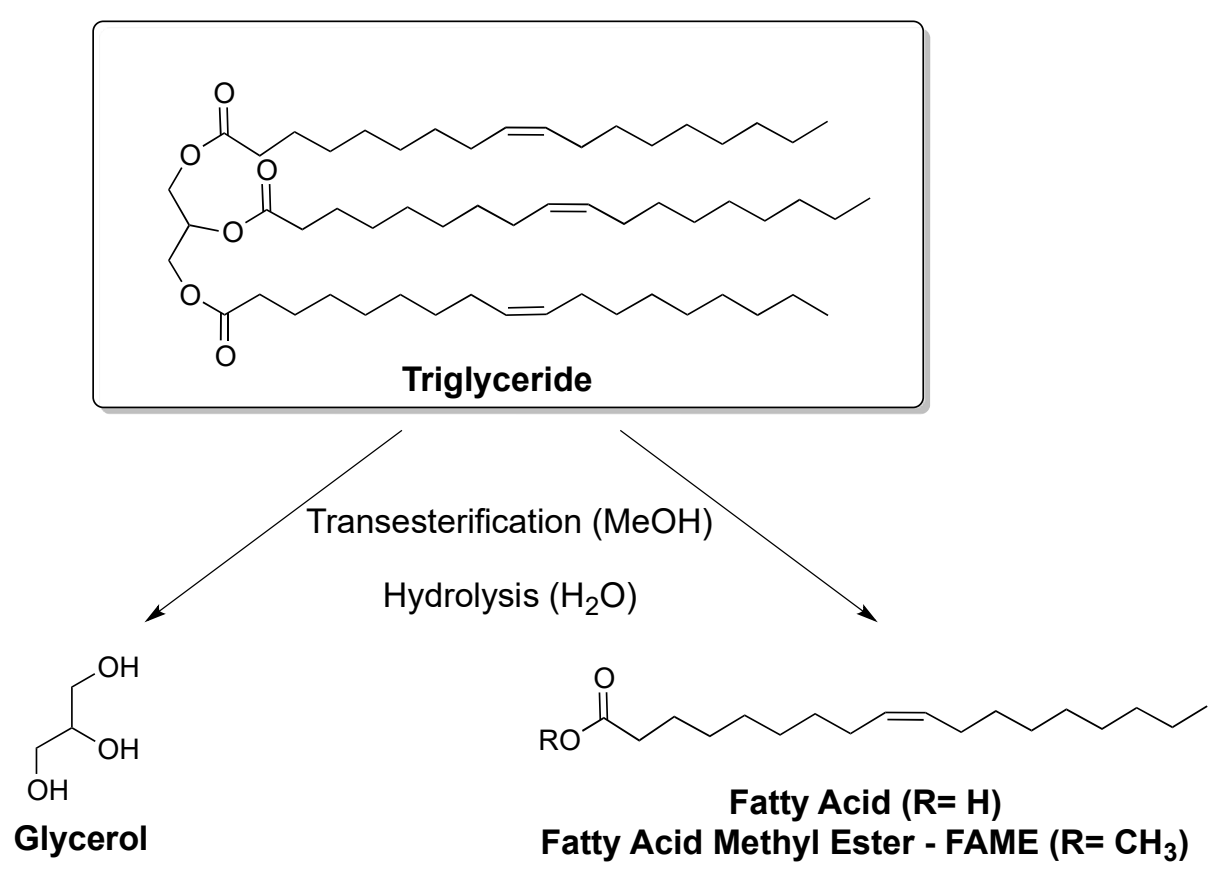

BIO-SOURCED VALUABLE MONOMERS 
Figure 59. Vegetable oils as a renewable resource for polymer industry

\section{B. Triglyceride-based polymers}

Some vegetable oils comprising castor oil or vernonia oil contain reactive functional groups such as hydroxyl or epoxide moieties that can be readily exploited for polymerization. However, the low content of available functions conferred to the final materials limited applications and weakened thermomechanical properties. ${ }^{258,259}$ As mentioned above, triglycerides can be chemically modified to increase and diversify functional groups, leading to many well-known synthetic routes to prepare vegetable oil-based polymers. ${ }^{260-262}$ In most cases, triglycerides are copolymerized with rigid comonomers with the aim of improving material performances. Two main families, comprising polyurethanes and polyepoxide thermosets, will be discussed by the next section. Again, the presented content is not exhaustive and aims at refer the major recent innovations in this field.

\section{Polyurethanes (Polyols) from triglycerides}

Polyurethanes are classically prepared from polyols and polyisocyanates and cover a large range of applications in adhesives, elastomers, coatings and foams. ${ }^{261,263-265}$ Interestingly, only a few vegetable oilbased polyisocyanates ${ }^{266}$ have been described in the literature in comparison with the wide range of reported renewable polyols. ${ }^{266,267}$ In most cases, triglycerides and more specifically long aliphatic chains, play the role of soft amorphous segments, conferring to PU a high flexibility. The comonomer is often chosen as hard segment in order to balance the composition and to tune the material thermo-mechanical properties. $^{268}$

First studies involving vegetable oil-based PUs were carried out on castor oil which presents hydroxyl moieties at the natural state and which is a non-food grade feedstock. ${ }^{266}$ Besides, a large number of studies reported the possibilities to functionalize triglycerides for PU precursor synthesis.

Figure 60 highlights the five main routes to polyols engaging chemical modifications of triglycerides. The epoxidation of the available double bonds, followed by the ring opening of the epoxides is one of the most described pathways in the literature (Figure 60-(1)). ${ }^{260,269-271}$ The double bonds are epoxidized using hydrogen peroxide and the produced oxirane groups are reacted with a nucleophilic ring-opening agent with or without catalyst to give the final polyol. Kessler et al. ${ }^{267}$ reviewed the recent advances involving the ring opening reaction. Many nucleophiles such as acetic acid, amines methanol, glycol, 1,2propanediol, 1,3-propanediol, diethylene glycol, poly(ethylene glycol) and even water have been used to ring-open oxiranes. ${ }^{268,271-276}$ Such a reaction is generally catalyzed with Lewis acids or inorganic acids like phosphoric or sulfuric ones. ${ }^{272,277}$ Vegetable oil-based polyurethanes prepared from this route exhibited properties ranged from soft elastic to hard materials. Some very interesting features such as specific optical properties, biocompatibility, antibacterial feature or shape memory could be also obtained. ${ }^{278-282}$

Vegetable oil-based polyols can also be synthesized from the transesterification/transamidation reaction between ester functions of triglycerides and alcohols/amines (Figure 60-(2)). ${ }^{283-288}$ Glycerol is often used as transesterification agent to prepare such polyols. ${ }^{289}$ Besides, the number of hydroxyl groups present on the polyol can be increased by reacting castor oil, rich in natural hydroxyl moieties, with one transesterification agent. This strategy can circumvent the presence of unreactive aliphatic chains, enabling to obtain PU with strengthen thermo-mechanical properties. ${ }^{290}$ The PU so-formed exhibit a large 
palette of properties with respect to the oil, the transesterification/amidation agents and the diisocyantes used. $^{267}$

The third route consists in the hydroformylation of double bonds in aldehydes using $\mathrm{CO} / \mathrm{H}_{2}$ gas under catalysis (mostly cobalt or rhodium based catalysts) that are subsequently hydrogenated under $\mathrm{H}_{2}$ (Figure 60-(3)). ${ }^{291}$ This last step leads to the formation of primary hydroxyl groups known to be more reactive towards polyaddition with diisocyanates. Commercially available polyols were synthesized by Dow Chemical Co. and BASF ${ }^{292}$ following the hydroformylation/reduction strategy on fatty acid methyl esters (Renuva TM polyol) and castor oil (Lupranol Balance 35) respectively. However, one study showed that this route displayed a main drawback as the PU films prepared were black. ${ }^{293}$

The ozonolysis of the double bounds followed by a reduction step into aldehyde, further reduced into hydroxyl functions has been investigated by several research groups in order to prepare more reactive polyols with primary hydroxyl moieties (Figure 60-(4)). Besides, ozonolysis leads to fatty acid chain cleavage and hydroxyl formation at the chain end, resulting in more rigid PUs when copolymerized with diisocyanate. ${ }^{294}$ It is noteworthy to mention that this route limits the number of hydroxyl groups to three and consequently decreases the crosslinking density.

A last method to produce polyols from vegetable oils is the use of thiol-ene click chemistry on double bonds (Figure 60-(5)). Indeed, this route involves a mild synthesis displaying high reaction rate, high atom economy, high yields and low reaction times. The reaction of thiols such as 2-mercaptoethanol on vegetable oil insaturations have already been reported by several research groups to prepare polyols. ${ }^{295-301}$ This strategy enables the simple access to other chemicals such as vegetable oil-based polyamines by reacting cysteamine on double bonds for instance. ${ }^{302,303}$ Using thiol-ene addition, another strategy has been developed and involves the preparation of mercaptanized vegetable oils, enabling a further reaction with an unsaturated compound. ${ }^{297,304}$

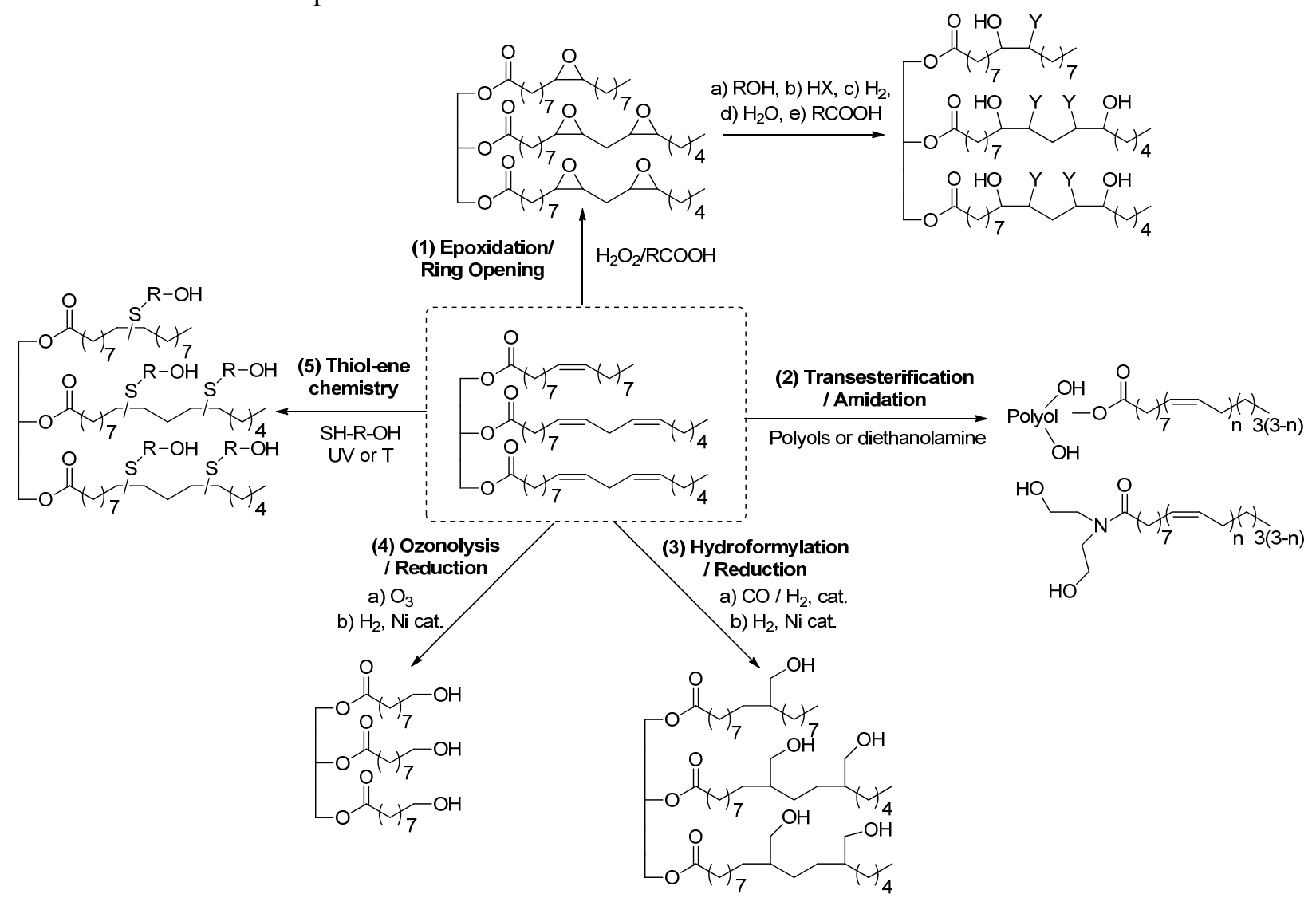

Figure 60. Strategies to prepare polyols from triglycerides 
It is noteworthy to mention than in the context of sustainable chemistry, the synthesis of non-isocyanate thermoset polyurethanes from vegetable oils has been investigated. Many works reported the preparation of poly(cyclic carbonate)s via the epoxidation of triglyceride carbon-carbon double bonds, followed by a carbonation under pressurized $\mathrm{CO}_{2}$ before being polymerized with polyamines. ${ }^{305}$ This synthetic strategy will be further discussed.

\section{Polyepoxide networks from triglycerides}

The scope of this chapter is limited to epoxidized vegetable oils precursors that can be cross-linked with several hardeners such as amines, acids and anhydrides to prepare epoxy networks. However, the synthesis of vegetable oil-based polyamines and their use in epoxy network syntheses is not included in this scope.

Commercially available epoxy resins are commonly prepared from bisphenol-A and epichlorohydrin whose the use is more and more restricted due to their inherent toxicity. ${ }^{306}$ Significant efforts have been made in the last decade to substitute classical epoxy precursors by vegetable oil-based ones. The latter can naturally contain oxirane moieties as it is the case of vernonia oil, or can be epoxidized following several methodologies.

The first route, probably the most employed, consists in the double bounds epoxidation with hydrogen peroxide as oxidative agent. This chemistry is sensitive to the double bound configuration and can lead to side reactions with subsequent low epoxide content. ${ }^{306}$ Nonetheless, many studies have described the epoxidation of castor, soybean, grapeseed, canola or linoleic oils via this methodology. ${ }^{307-310}$ Other routes using peroxides and involving metal catalysts (rhenium, oxomolybdenum...) ) $^{311,312}$ and enzymes (ex: Novozym $\left.{ }^{\circledR} 435\right)^{313}$ were also investigated to increase yields and selectivity. One of the main producers of epoxidized vegetable oils is the company Arkema who has developed the production of epoxidized soybean oil (Figure 61) under the name VIKOFLEX ${ }^{\circledR}{ }^{314}$ Also, it is important to note that epoxidized vegetable oils are used as additives or plasticizers in paints, coatings or PVC. ${ }^{315}$

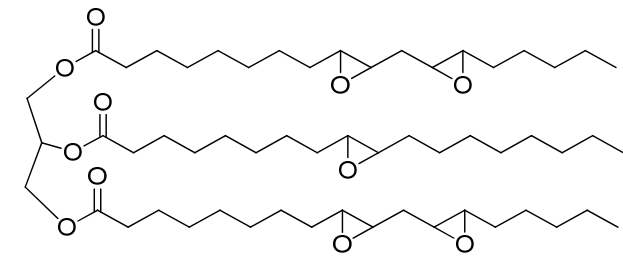

Figure 61. Epoxidized soybean oil structure

Some research groups performed the self-polymerization of epoxidized soybean or castor oils using specific thermally latent catalysts such as N-benzylpyrazinium hexafluoroantimonate or $\mathrm{BF}_{3} . \mathrm{OEt}_{2} .{ }^{316,317}$ Nonetheless, these epoxy precursors are generally copolymerized with different crosslinking agents. For instance, amines, acids and anhydrides have been used as hardener to prepare vegetable oil-based epoxy resins.

Stemmelen et al. described the polymerization of a linear bio-based diamine from grapeseed oil fatty acid and polyamines from the same oil with epoxidized linseed oil. The fully vegetable oil-based epoxy resins exhibited good mechanical properties but lowered chemical resistance due to the poor crosslinking density, correlated to the low monomer reactivities. ${ }^{318,319}$

In order to tune the epoxy network properties, epoxidized vegetable oil are commonly mixed with commercial and petroleum-based epoxy precursors. Several papers reported the impact of the epoxidized vegetable oil content in the epoxy resin formulation, containing also bisphenol-A derivative, on the network properties. These studies demonstrated that by increasing the amount of triglyceride-based 
epoxides, flexibility and elongation at break increased but chemical resistance, crosslinking density as well as thermo-mechanical properties were lowered. ${ }^{320-322}$

Various bio-based diacids were employed as crosslinking agent in the formulations. A blend of epoxidized soybean oil and diglycidyl ether of bisphenol A was cured using the fatty acid-based sebacic acid that exhibited higher efficiency than classical amine hardeners. ${ }^{323}$ Also, another group conducted the reaction between epoxidized soybean oil and the bifunctional citric acid in order to provide fully biobased epoxy networks. ${ }^{324}$ Moreover, Mathary et al. used the diacid belonging to the Priplast ${ }^{\circledR}$ range developed by CRODA as crosslinking agent for epoxidized linseed oil. The resulting epoxy networks obtained under a base-catalyzed process displayed both good water resistance and thermal stability. ${ }^{325}$

Finally, other studies investigated the crosslinking of several epoxidized vegetable oils with anhydrides such as phthalic anhydride, ${ }^{326}$ maleic anhydride, ${ }^{327}$ methyl hexahydrophthalic anhydride, ${ }^{328}$ methyl tetrahydrophthalic anhydride ${ }^{329}$ and methyl nadic anhydride. ${ }^{330}$ One interesting study highlighted the possibilities of tuning epoxy network properties by curing an epoxidized cottonseed oil with the appropriate blend of methyl nadic anhydride and dodecenyl succinic anhydride, which bring respectively rigidity and flexibility. ${ }^{331}$

\section{Other triglycerides-based polymers}

The high density of functionalities imparted by triglycerides enables a plethora of polymerization processes and subsequent materials. Some of them require prior triglyceride modification. Figure 62 summarizes some interesting polymerization routes to access cross-linked vegetable oil-based polymers. 


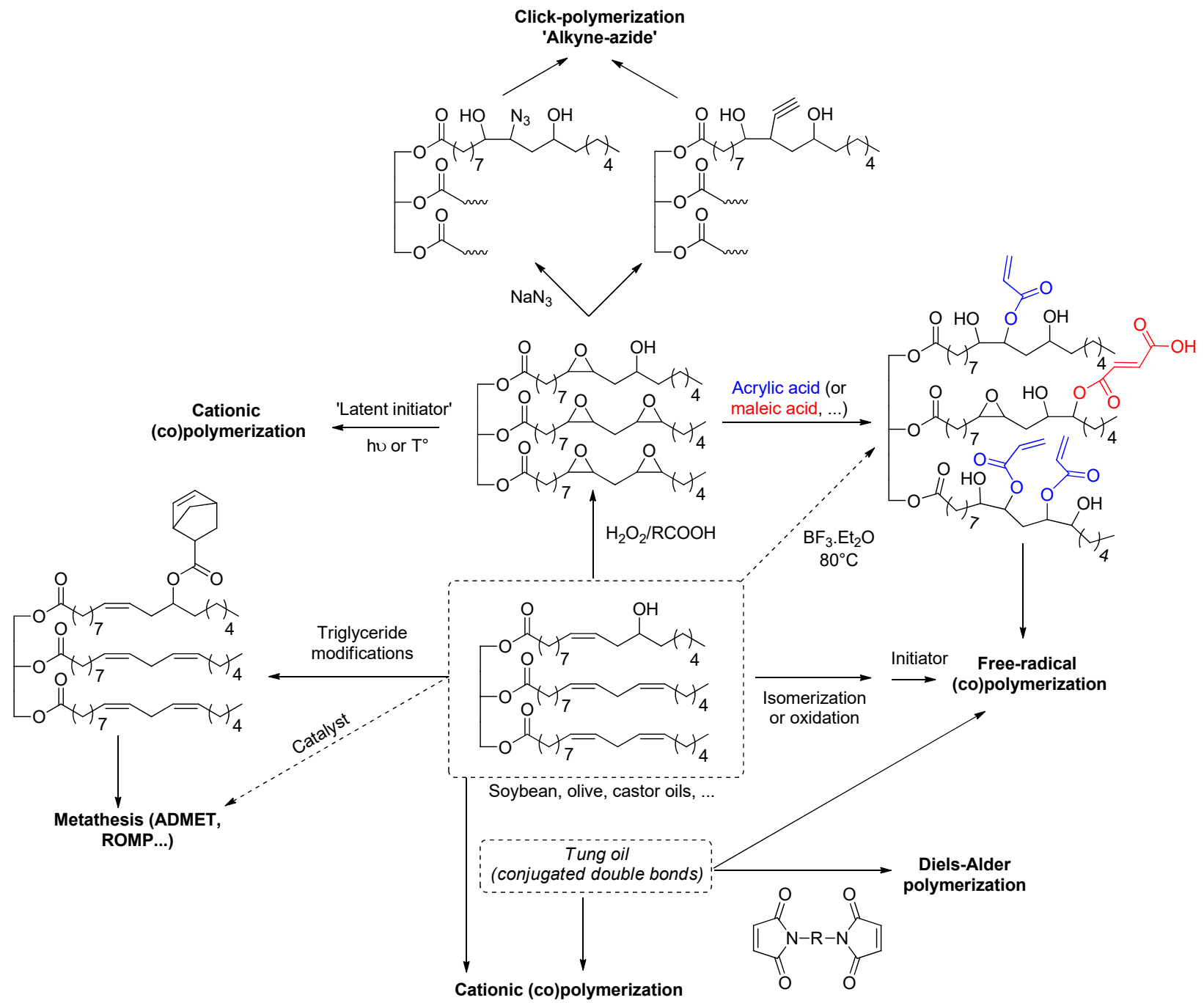

Figure 62. Triglyceride-based monomers for thermoset polymers

At the industrial scale, oxypolymerization has been largely used to modify vegetable oils into 'drying oils' ${ }^{332-335}$ Indeed, after an exposure to air and oxygen, $\mathrm{C}=\mathrm{C}$ double bonds composing oils (linseed, tung or soybean) generate radicals and can be oxidized to form peroxides that can cross-link oils by radicals recombinaison. These modified oils have been widely used as free-radical macroinitiator, as linoleum floor covers or as binders in oil paints and varnishes thanks to their high viscosity. ${ }^{336-340}$

Besides, the majority of triglycerides are composed of unsaturated and unconjugated fatty acids with limited double bound reactivity, limiting the access to effective free radical polymerizations. One exception is made for tung oil, mainly composed of $\alpha$-eleostearic acid, which comprises a naturallyoccurring conjugated triene (Figure 63). This readily polymerizable oil has already been copolymerized with styrene and divinylbenzene $e^{341,342}$ following a thermally-initiated free radical process.

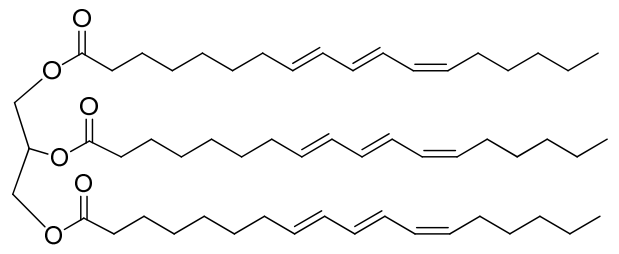

Figure 63. Hypothetical tung oil structure ( $\sim 85 \% \alpha$-eleostearic acid) 
In order to obtain more reactive conjugated monomers, other vegetable oils were first isomerized ${ }^{343}$ before being copolymerized with various comonomers. ${ }^{344,345}$ For instance, Larock and co-workers synthesized bio-based thermosets from the free-radical copolymerization of conjugated linseed oil with acrylonitrile and divinylbenzene. ${ }^{34,345}$

Another strategy involving the ring-opening of epoxidized vegetable oils by acrylic acid ${ }^{346-349}$, maleic anhydride ${ }^{350}$ and other (meth)acrylated compounds ${ }^{351}$ has been widely reported in order to introduce more reactive double bonds onto triglycerides. Recently, Zhang et al. proposed a new route to access acrylated soybean oil from raw triglycerides in a one-step procedure using $\mathrm{BF}_{3} . \mathrm{Et}_{2} \mathrm{O}$ as catalyst, avoiding the oxirane ring-opening step. ${ }^{352}$ Generally, the reported (meth)acrylated oils are (co)polymerized following a free radical polymerization initiated by heat, UV or chemical initiators. Some of these polymers were described to be good candidates for pressure sensitive adhesives ${ }^{353-355}$ or even for electronic materials. ${ }^{351}$ It is noteworthy to mention that acrylated epoxidized soybean oils have been extensively used in surface coatings and have already been commercialized by UCB Chemicals Company (Ebercryl 860). ${ }^{350,356}$

Nonetheless, vegetable oils with less reactive carbon-carbon double bound have been copolymerized with styrene without prior modifications, in order to blend bio-based content with styrenic materials to subsequently improve the initial mechanical properties. ${ }^{314}$

Taking advantage of the high nucleophilicity of carbon-carbon double bounds present on triglycerides, cationic (co)polymerizations of vegetable oils have been carried out using generally protic or Lewis acids as initiators. Thus, different oils have been copolymerized via a cationic mechanism with divinylbenzene and styrene. ${ }^{357-362}$ Soybean oil was also copolymerized with dicyclopentadiene to form partly bio-based thermosets. ${ }^{363,364}$ Also, cationic polymerization of soybean oil in supercritical $\mathrm{CO}_{2}$ has been undertaken using $\mathrm{BF}_{3} . \mathrm{Et}_{2} \mathrm{O}$ as catalyst. ${ }^{365}$ The obtained liquid soy polymer was found to be a good candidate for medical lubricants. Using the same catalyst, tung oil was found to be highly reactive towards cationic polymerization thanks to its double bond conjugation. ${ }^{366}$ Additionally, epoxidized vegetable oils can be cationically polymerized using 'latent initiators' such as benzylpyrazinium salts under heating or photoirradiation. $^{316,367-371}$

Milder chemistries such as metathesis reaction, azide-alkyne Huisgen cyclo-addition or Diels-Alder reactions have been applied to triglycerides with the aim to prepare polymeric materials.

Larock's group investigated the acyclic diene metathesis (ADMET) of various vegetable oils using the first generation of Grubbs catalyst, taking advantage of the double bonds abundancy in triglycerides. ${ }^{372}$ Further studies were conducted on the particular case of metathesized soybean oils. ${ }^{373,374}$ Besides, vegetable oils can be modified before being polymerized via metathesis techniques. Indeed, rubbery thermosests could be obtained by processing the ring-opening metathesis polymerization (ROMP) of a norbornene-functionalized castor oil with cyclooctene. ${ }^{375}$ Also, a commercially available mixture of norbornenyl-functionalized linseed oil and cyclopentadiene oligomer crosslinkers sold under the name Dilulin (Figure 64) has been polymerized by ROMP with dicyclopentadiene ${ }^{376}$ or polycyclic norbornenebased monomers. ${ }^{376}$

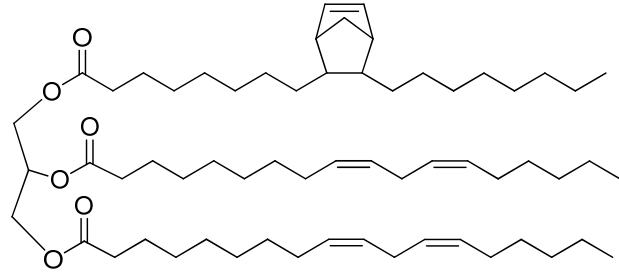

Dilulin oil fraction

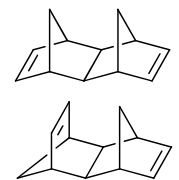

Crosslinkers

Figure 64. Blend composition of Dilulin 
Furthermore, Petrovic's group carried out the syntheses of azide-functionalized triglycerides and subsequently performed the 'click' coupling (also called Huisgen cyclo-addition) with dialkyne or polyalkynes comprising alkynated oils. ${ }^{377,378}$

Lastly, an interesting polymerization, through irreversible Diels-Alder coupling, has to be mentionned. Thermosets were obtained by click coupling between tung oil conjugated double bounds and aliphatic or aromatic bismaleimides. ${ }^{379,380}$ Contrarily to the furan-maleimide Diels-Alder reaction, the coupling was irreversible even when high temperatures were applied.

Despite the high functionality density imparted by the triglycerides, the polymerization of such chemicals results in lowered material properties. Indeed, due to the variable structure of triglycerides in a same oil sample, the relationship between structures and properties is difficult to make and the reproducibility is almost impossible to target. Therefore, building blocks-composing triglycerides, i.e. fatty acids and glycerol have been respectively used as monomers for well-defined polymer syntheses.

\section{Glycerol-based polymers}

The glycerol production has been widely increased in the past few years due to the development of fatty acid methyl esters production. Indeed, this compound of interest is produced in large quantity from the triglyceride transesterification, and used as fuel additive (biodiesel). Therefore, an extensive effort has been done in the academic and industrial research in order to valorize the glycerol, which is the byproduct of this reaction. Additionally, glycerol is a cheap and available intermediate that can be derivatized into numerous chemicals thanks to its multifunctional character. The Figure 65 sums up the most prevalent chemicals obtained from glycerol transformations. For instance, the etherification, chlorination and hydrogenolysis of glycerol are respectively leading to oligo/polyglycerols, epichlorohydrin and 1,3-propanediol. ${ }^{381}$ The main advantage of these intermediates is their biocompatibility suitable for biomedical applications. ${ }^{382}$

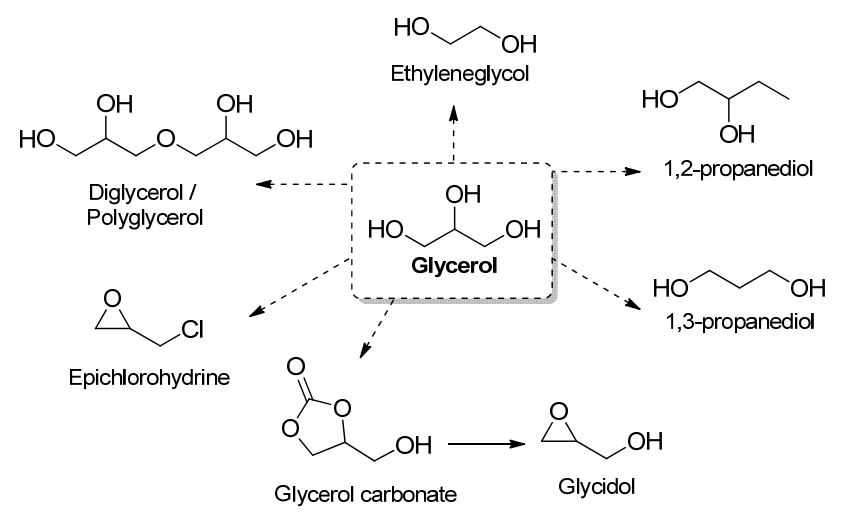

Figure 65. Most common molecules obtained from glycerol chemical transformations

The syntheses of glycerol derivatives will not be detailed in this part. Special attention will be paid to the most relevant polymers obtained from glycerol or derivatives.

\section{Polyglycerol (including hyperbranched polyethers)}

Glycol ethers such as ethylene glycol are commonly produced from fossil resources and are used mainly for their coalescence properties in inks, paints or solvent. ${ }^{383}$ Due to their toxicity and CMR character, 
industrials tend to substitute these additives by oligo- and polyglycerols, preferentially derived from renewable resources. By varying the degree of branching or the degree of polymerization, a wide palette of oligo- and polyglycerols with tunable properties is available. For instance, some of these polymers have already been employed as plasticizers, surfactants, lubricants or in cosmetic and pharmaceutical industries. ${ }^{384-394}$

Lemaire and co-workers reported the different approaches to polyglycerol. They highlighted industrial routes such as the reaction between epichlorohydrin and allylic alcohol in 5 steps or the anionic polymerization of glycidol. ${ }^{395}$ However, these routes represent a limitation in terms of atom economy and toxicity. Therefore, new eco-friendly etherification processes leading to polyglycerol and starting from raw glycerol or glycerol derivatives have emerged.

Glycerol can be self-polymerized following two different mechanisms producing linear or hyperbranched oligo- or polyglycerol. These two polymerizations involve respectively the primary hydroxyl functions and both primary and secondary one, depending on the catalysts employed. ${ }^{396}$ When the etherification occurs between primary hydroxyl groups, linear polyglycerols are produced as depicted in Figure 66-(A), whereas hyperbranched polyglycerol schematized in Figure 66-(B) can be obtained through the etherification of both primary and secondary hydroxyl groups. Many acidic or basic, homogeneous or heterogeneous catalysts have been tested. As reported by Martin et al., ${ }^{396}$ the catalyst efficiency was closely linked to their nature and structure. Regarding the linear polymerization of glycerol, one of the most profitable catalysts remains potassium carbonate that can be well solubilized in glycerol at high temperature, improving the etherification conversion. ${ }^{397}$

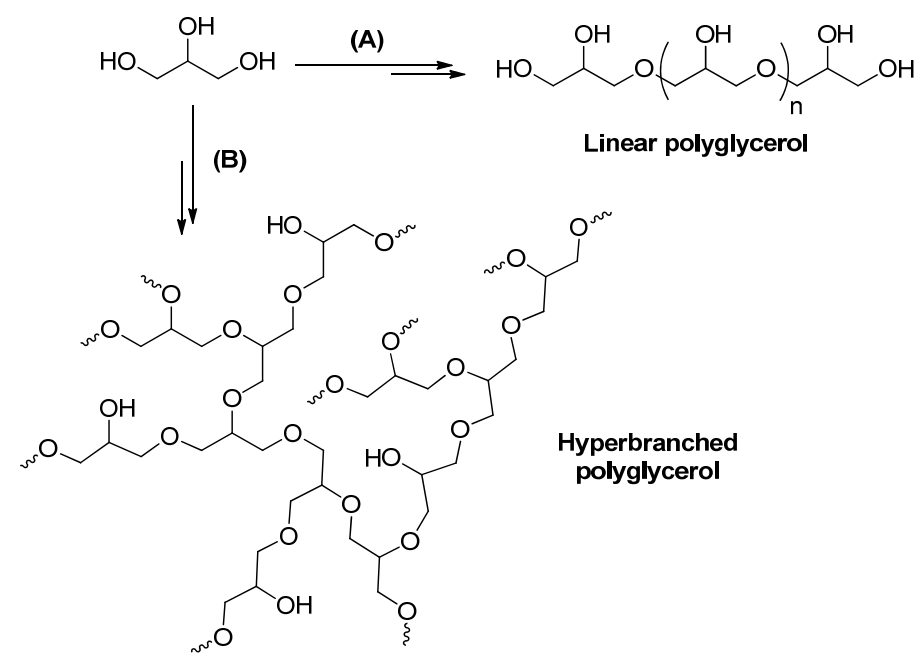

Figure 66. Homopolymerization of glycerol leading to (A) linear or (B) hyperbranched polyglycerols

The main limitations of glycerol etherification remain high temperatures, high energetic costs and basic conditions employed during syntheses. ${ }^{382}$ To tackle this issue, milder routes have been investigated to access polyethers. The preparation of polyglycerols from glycerol carbonate, itself derived from glycerol has also been carried out under microwave irradiation, enabling the in situ formation of glycidol by decarboxylation. ${ }^{398}$ Another interesting monomer derived from the hydrogenolysis of glycerol is ethylene glycol that can be polymerized to obtain the widely used poly(ethylene glycol). ${ }^{381}$

Going further, a plethora of linear polyethers from functionalized glycidyl ethers derived from glycerol has been investigated and displayed an excellent biocompatibility. ${ }^{382}$ Besides, mono-, oligo- and polyglycerols can be modified by transesterification with fatty acid ethyl esters in order to target applications such as cosmetic and food additives, detergents or lubricants. ${ }^{399-401}$ 


\section{Hyperbranched polyesters (HPBEs)}

Additionally to the hyperbranched polyethers synthesized from glycerol, other types of glycerol-based hyperbranched polyesters (HBPEs) have been designed to introduce the biodegradability feature. Two main strategies have been investigated to access glycerol-based HBPEs via either the copolymerization of glycerol with diacids (or diesters) or the self polycondensation of a glycerol-based $\mathrm{AB}_{\mathrm{n}}$-type monomer.

The first route consisting in the copolymerization of glycerol with a comonomer has been widely described in the literature. Many bio-based hyperbranched copolyesters has been prepared using classical catalyst or enzymes such as the lipase Candida Antarctica (CALB), to process the polycondensation between glycerol and various renewable diacids such as succinic, adipic, citric acids or even anhydrides.

Wyatt and Strahan ${ }^{402}$ synthesized hyperbranched polyesters from the polycondenstaion between glycerol and various diacids of different chain lengths (succinic, glutaric and azelaic acids) with dibutyltin oxide as transesterification catalyst. The degree of branching and the diacid structure were correlated and showed that the shortest glutaric acid led to the highest degree of branching (85.6\%) in comparison with the long-chain azelaic diacid (13.9\%).

Another study described the copolymerization between glycerol, 1,8-octanediol and adipic acid at $70^{\circ} \mathrm{C}$, under vacuum, using CALB as catalyst (Figure 67). The regioselectivity of the enzyme permitted the preparation of linear copolyesters when reaction time was limited (18h). Nonetheless, as the latter was extended to $42 \mathrm{~h}$, hyperbranched copolyesters resulting from the reaction of secondary hydroxyl groups with acid moieties were obtained. ${ }^{403}$ This work highlighted the importance of condition reactions on the copolyester final architecture.

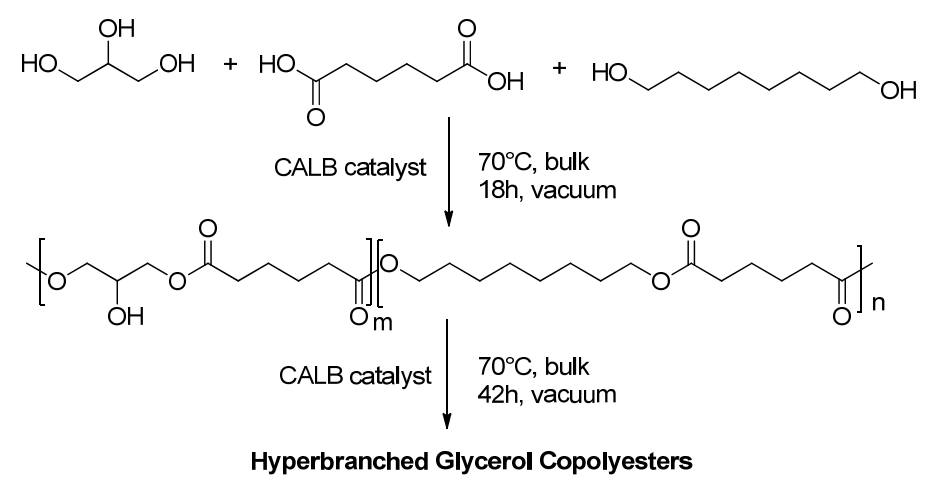

Figure 67. Enzymatic copolymerization of glycerol, adipic acid and 1,8-octanediol. ${ }^{404}$

In the same category, polyglycerol sebaçate prepared from the polycondensation between ricinoleic-based sebacic acid and glycerol has gained academic research interest. Indeed, this material is biocompatible and bioresorbable, enabling potential applications in the biomedical field. ${ }^{405}$

Besides, citric acid is a low cost and trifunctional monomer conferring anti-microbial and biodegradable properties when polymerized. The preparation of glycerol- and citric acid-based hyperbranched polyesters was carried out using an effective and catalyst-free $\mathrm{A}_{3}+\mathrm{B}_{3}$ melt polycondensation (Figure 68). ${ }^{406}$ The authors studied the influence of the glycerol stoichiometry on the degree of branching and the biodegradability and proved the high potential of such systems for drug delivery. Tisserat et al. described the same synthesis using microwave irradiation. ${ }^{407}$ Thanks to their biocompatible characters, those biobased hyperbranched polymers could be potential candidates for tissue engineering applications 


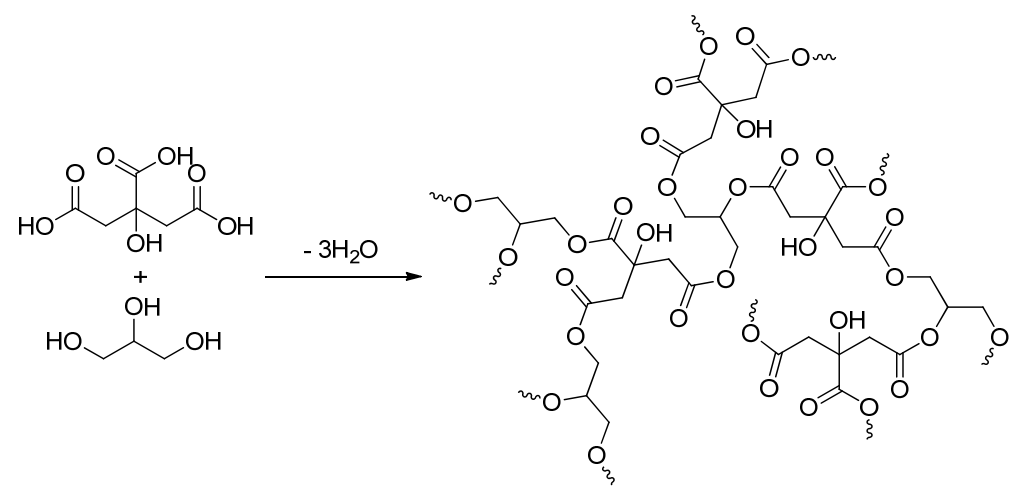

Figure 68. Hyperbranched polyester from glycerol and citric acid

Another interesting example is the bulk preparation of hydrosoluble HBPEs from glycerol and maleic anhydride. ${ }^{408,409}$ Improved water solubility was observed when pentaerytritol was added as a core molecule in the system. The HBPEs was then reacted with acrylic ester to form acrylic ester latex film with improved mechanical properties and minimized water adsorption. In another study, maleic anhydride was also copolymerized with glycerol and succinic acid for the preparation of HBPEs as poly(lactic acid) reinforcement.

The synthesis of HBPEs has also been carried out via the self-polycondensation of an $\mathrm{AB}_{\mathrm{n}}$-type monomer. Meier's and Li's groups both prepared the fatty acid- and glycerol-based $\mathrm{AB}_{2}$-type monomer depicted in Figure 69-(A) by reacting 1-thioglycerol and methyl 10-undecenoate via a thiol-ene addition. The first group prepared HBPEs with and without glycerol as core molecule, at $120^{\circ} \mathrm{C}$ using TBD as catalyst. Molecular weights were ranging between 3500 and 4400 g.mol ${ }^{-1}(\bigoplus=1.9-2.8)$. Higher HBPEs molecular weights $\left(\mathrm{Mn}=11400-60400\right.$ g. $\left.\mathrm{mol}^{-1}, \mathrm{D}=5.2-25.3\right)$ were obtained by the second group using metallic catalysts such as $\mathrm{Ti}(\mathrm{OBu})_{4}$ or $\mathrm{Zn}(\mathrm{OAc})_{2}$ and working at $160-170^{\circ} \mathrm{C}$.

Parzuchowski et al. reported the synthesis of another $\mathrm{AB}_{2}$-type monomer (Figure 69-(B)) for the preparation of HBPEs that can be easily degraded by hydrolysis or alcoholysis, making them good candidates for recyclable materials. ${ }^{408}$
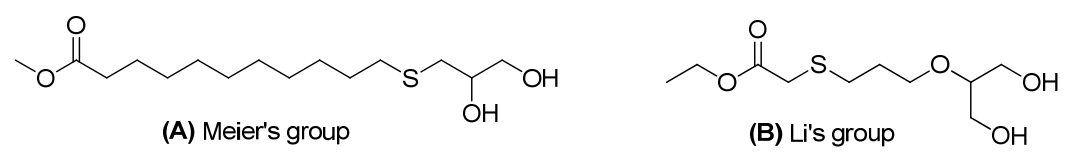

Figure 69. $\mathrm{AB}_{2}$-type glycerol based monomers from (A) Meier's and (B) Li's groups.

\section{Glycerol-based linear polyesters}

The examples of linear glycerol-based polyesters are very limited. One of the only specimens comes from the copolymerization between a diacid and 1,3-propanediol that is readily obtained from the selective hydrogenolysis of glycerol, using specific metallic catalysts. ${ }^{381}$ At the industrial scale, this synthon of interest is copolymerized with terephtalic acid to produce a bio-based polyester patented under the brand SORONA ${ }^{\circledR}$ from Dupont or CORTERRA ${ }^{\circledR}$ from Shell. ${ }^{410}$

Besides, few papers reported the synthesis of linear poly(hydroxyester)s from the multifunctional glycerol and a diacid (or diester). Their main objective was to adjust the reaction conditions (stoichiometry, catalysts, temperature) in order to limit side reactions such as branching or crosslinking. For instance, Gross and coll. ${ }^{411}$ demonstrated that linear poly(hydroxyester)s with very low degree of branching could 
be synthesized from glycerol and oleic diacid by employing the immobilized Candida antarctica lipase B as transesterification catalyst. Using the same enzyme, Kobayashi and coll. described two epoxidized aliphatic polyesters from the reaction between glycerol, fatty acids and divinyl sebaçate. ${ }^{412,413}$ The epoxidation was carried out using hydrogen peroxide before or after copolymerization depending on the strategy adopted. The linear polyesters were then thermally cured to obtain biodegradable polyepoxide networks.

\section{Glycerol-based polyurethanes}

Regarding the classical polyurethane chemistry, all the glycerol-based polyols including 1,3-propanediol, polyglycidol or glycerol it-self can be copolymerized with polyisocyanates to obtain many varieties of polyurethanes with tunable properties depending on the polyol structure. The numerous examples reported in the literature show a real attraction for the renewable and low-cost glycerol. Some recent studies report the preparation of such polyurethanes. For instance, Li et al. prepared a crude glycerolbased polyols by reacting glycerol at $150^{\circ} \mathrm{C}$ under vacuum for $5 \mathrm{~h}$ without catalyst. ${ }^{414}$ The subsequent foaming in presence of methylene diisocyanate, surfactant and water was performed and the resulting foams demonstrated improved cell morphologies thanks to the presence of residual fatty acid derivatives present in crude glycerol. Another research group substituted petroleum-based polyols by polyglycerol in their rigid polyurethane foam formulation and demonstrated the improved mechanical properties of the renewable materials. ${ }^{415}$ Besides, $\mathrm{Hu}$ et al. used crude glycerol based-polyols to prepare water-bone polyurethane dispersions. ${ }^{416}$ Glycerol can also be used as source of polyol in foam formulations. ${ }^{417}$

In the context of sustainable chemistry, reducing reagent toxicity and using bio-based chemicals have been two major challenges for the chemistry of the last decades. To feed the needs in the polyurethane chemistry domain, research about non-phosgene and non-isocyanate polyurethanes (NIPU) has been stepped up. The most promising route is the polyaddition between poly(cyclic carbonate)s and polyamines leading to poly(hydroxyurethane) formation (Figure 70). As reviewed by Maisonneuve et al., bio-based cyclic carbonates can be prepared from epichlorohydrin, glycerol carbonate or either diglycerol, making glycerol an attractive synthon. ${ }^{418}$

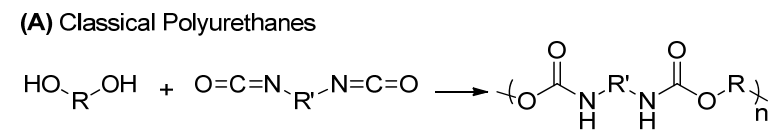

(B) Poly(hydroxyurethane)s

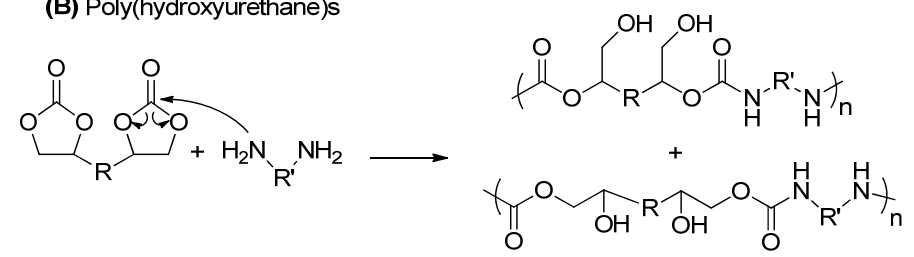

Figure 70. Synthesis of (A) classical polyurethanes and (B) poly(hydroxyurethane)s

\section{Glycerol-based polyepoxide networks}


The great majority of epoxy monomers are produced from the reaction between epichlorohydrin and hydroxylated compounds. ${ }^{419}$ The reaction is generally conducted in aqueous solution of sodium hydroxide, using epichlorohydrin in excess. As already discussed, the most popular remains the diglycidyl ether of bisphenol-A (DGEBA). Petroleum-based epichlorohydrin is usually produced from the chlorohydrination of allyl chloride. ${ }^{420}$ Solvay patented the synthesis of bio-based epichlorohydrin from glycerol (Figure 71) under the brand EPICEROL ${ }^{\circledR}$, which is produced at a level of $100 \mathrm{kt}^{\text {per year. }}{ }^{421}$ Therefore, the bio-based weight content of DGEBA reached $25 \%$ when the bisphenol-A glycidation was carried out with glycerol-based epichlorohydrin. The recent review released by Auvergne and coworkers ${ }^{419}$ summarized the different epoxy monomers from biomass, including the ones synthesized from epichlorohydrin.

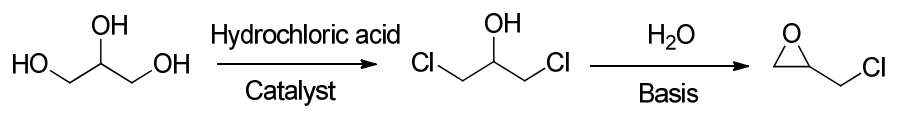

Figure 71. Glycerol chlorination to prepare bio-based epichlorohydrin (EPICEROL technology).

One of the first glycerol-based polyepoxide network was prepared by Barua et al. by reacting bisphenolA, glycerol and epichlorohydrin in aqueous basic solution. ${ }^{422}$ Besides, poly(epoxide) prepolymers, particularly aliphatic glycidyl ether ones, can be obtained from glycerol and are commonly produced at the industrial scale. For instance, the synthesis of epoxy precursors (Figure 72) from epichlorohydrin and glycerol has been patented by Dow Chemicals. The authors have developed the range POLYPOX ${ }^{\circledR}$ glycidyl ethers used in epoxy formulations as reactive diluent. ${ }^{423,424}$

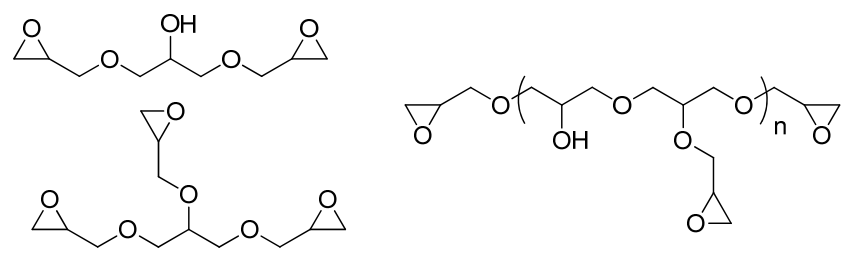

Figure 72. Glycerol-based poly(glycidyl ether)s.

\section{Fatty acid-based thermoplastic polymers}

Vegetable oils have been extensively used as intermediates for bio-based thermoset preparation, using raw oils or functionalized triglyceride monomers. However, as mentioned previously, the major problem encountered by this strategy is the heterogeneity of oil composition due to the random distribution of fatty acids within the triglyceride structure. Consequently, the correlation between the monomer structures and the polymer properties is hardly feasible. In light of this, fatty acids and their derivatives appears as the most promising building blocks for the synthesis of well-defined thermoplastic polymers with targeted and controlled properties. Indeed, fatty acids are available in a high-purity grade, enabling their use as monomers after chemical transformations. The latter are mainly conducted on the carbon-carbon double bonds along the fatty acid aliphatic chain and include oxidation, hydrogenation, hydroformylation, hydrovinylation, thiol-ene coupling, metathesis reaction, etc. Monomers, generally bifunctional, are thus designed to target a certain class of polymers with specific properties. Moreover, innovative properties can be conferred to vegetable oil-based polymers thanks to the structural difference existing between fatty acid- and petroleum-sourced chemicals. 
In the following parts, the preparation of the most preponderant fatty acid-based thermoplastic polymers comprising polyamides, polyurethanes, polyesters and polycarbonates will be developed. A special attention will be given to commercially available materials and to emergent and high-potential polymers.

\section{Polyamides}

Thermoplastic polyamides (PA) represent a class of high performance materials required in many sectors involving automotive, electrical or textile industries. Indeed, due to the presence of amide linkages, polyamides exhibit unique properties such as high melting points, high impact and tensile strengths, electrical insulation, chemical, heat, and abrasion resistances. PA are commonly synthesized following three routes which are (1) the polycondensation of dicarboxylic acids and diamines, (2) the selfpolycondensation of amino acids and (3) the ring-opening polymerization (ROP) of lactams (Figure 73). At the industrial scale, most aliphatic polyamides are prepared by melt-phase polycondensation and reach average molecular weight around 10000 g.mol ${ }^{-1} .{ }^{425,426}$

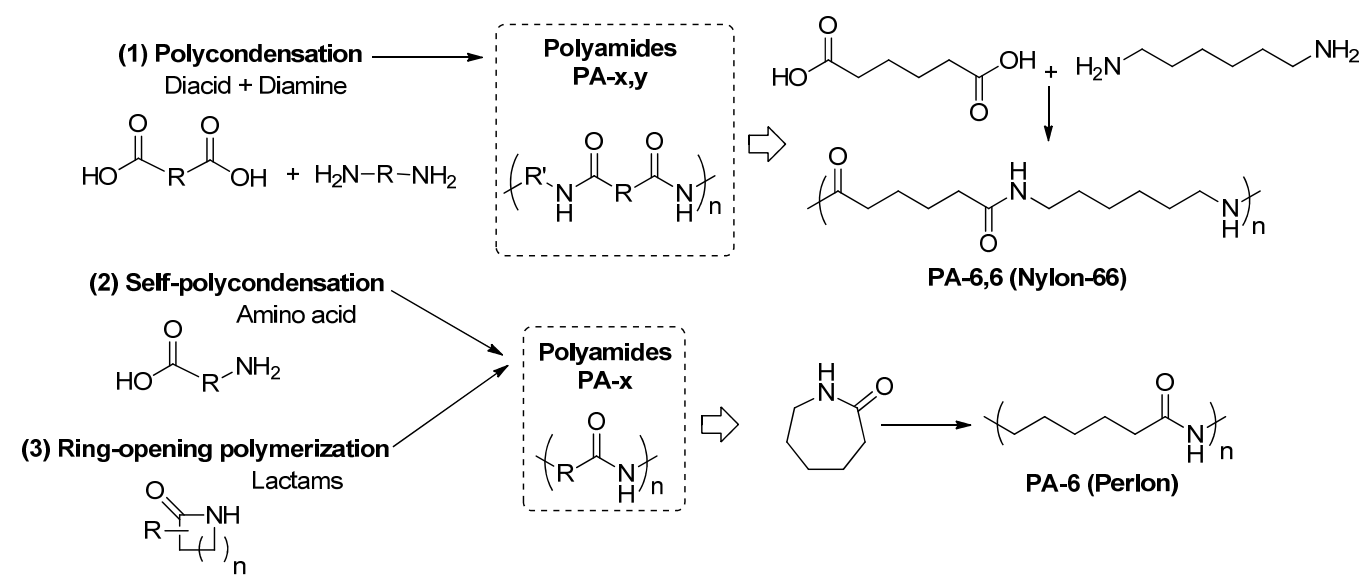

Figure 73. Different routes towards polyamide syntheses.

Depending on the polymerization route employed, AABB- or AB-type polyamides are obtained resulting in different nomenclatures written respectively PA-x,y and PA-x where $\mathrm{x}$ and $\mathrm{y}$ represent the number of carbons between two nitrogen atoms. ${ }^{427,428}$ For instance, PA-6,6 (Nylon-66) and PA-6 (Polycaprolactam, Perlon) count for $85-90 \%$ of the commercialized petroleum-sourced polyamides and are respectively synthesized by DuPont from the polycondensation of adipic acid and hexanediamine and by IG Farben from the ROP of $\varepsilon$-caprolactam. With regard to polyamide syntheses, it is noteworthy to mention that AABB-type polymerization is much more challenging than AB-type polyamide preparation. Indeed, according to the Carothers's law, the stoichiometry deviation between diacids and diamines can lead to low molecular weights and weakened polyamide properties. Therefore, these polycondensations are generally performed following a "nylon salt solution" ${ }^{429}$ which consists in transforming the two monomers in their corresponding salts in order to target the exact stoichiometry before polymerizing them at elevated temperatures.

The huge potential of vegetable oils for the synthesis of bio-based polyamides comes from the available long carbon chains enabling the preparation of aliphatic diamines, diesters (or diacids) and amino esters. One of the most important sources of vegetable oil-based polyamides is the castor oil (Figure 74). On the one hand, ricinoleic acid can be extracted from the hydrolysis of triglyceride from castor oil. A 
subsequent pyrolysis gives access to the $\mathrm{C} 11$ undecenoic acid, which is an excellent building block for AB-type polyamide preparation. This synthon can be chemically modified via a bromination step and a treaTment with ammonia in 11-aminoundecanoic acid. For instance, the AB-type PA-11 commercialized by Arkema under the trade name Rilsan ${ }^{\circledR}{ }^{430-432}$ has been produced by the self-polycondensation of 11aminoundecanoic acid for more than 50 years. This famous fully bio-based polyamide is more resilient and thermally resistant than PA- 6 and PA-6,6 and is produced at the scale of about 21000 tons per year for high performance applications (catheters, automotive fuel lines, ...). On the other hand, alkali fission of ricinoleic acid with $\mathrm{NaOH}$ or $\mathrm{KOH}$ can produce sebacic acid, which has been extensively used as monomer for AABB-type polyamide preparation. For instance, various fatty acid-based polyamides have been synthesized from sebacic acid by different companies such as Rhodia (Technyl ${ }^{\circledR}$ eXten, PA-6,10), BASF (Ultramid ${ }^{\circledR}$ Balance, PA-6,10), Evonik (VESTAMID® Terra, fully or potentially bio-based PA10,10 and PA-10,12) ${ }^{433}$ and DSM (EcopaXX ${ }^{\circledR}$, PA-4,10). ${ }^{434}$ Due to the lack of commercial bio-based diamines, it is much more difficult to produce fully bio-based AABB-type polyamides than AB-type one. Nonetheless, sebacic acid can be either transformed in the corresponding diamine to reach fully bio-based polyamides as it is the case of PA-10,10 from Evonik. ${ }^{435}$

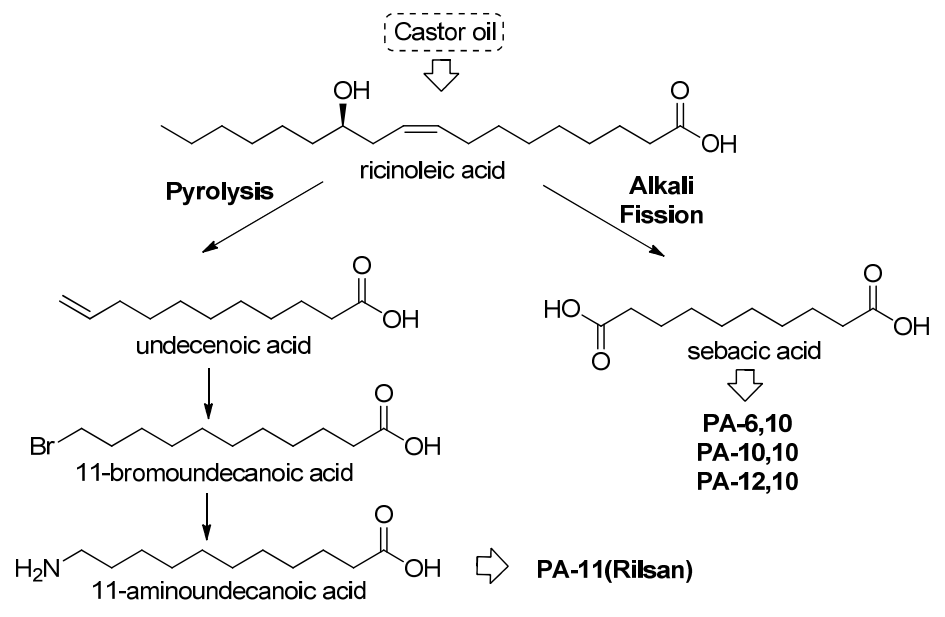

Figure 74. Castor oil as a platform for polyamide synthesis

As far as material properties are concerned, polyamides coming from castor oil have been used as matrix to reinforce glass, carbon, flax and cellulosic short fibers. ${ }^{436,437}$ The resulting composites displayed improved tensile strength, elongation at break and modulus of elasticity.

Depending on the synthesis and on the targeted application, polyamides can display various molecular weights. For example, PA-6,6 exhibits molecular weights ranging in between 25000 and 110000 g.mol ${ }^{-1}$ 438 contrarily to some fully bio-based polyamides that could only reach molecular weights up to 14700 g. $\mathrm{mol}^{-1}{ }^{439}$ More generally, melting temperatures and modulus increase with the density of amide linkages. For instance, PA- 6 and PA-11 display respective melting temperatures of 218 and $183^{\circ} \mathrm{C}$ demonstrating that PA- 6 requires higher thermal energy to break the more prominent H-bonding between amide functions. Mecking and coll. ${ }^{181}$ published a complete review reporting the specific properties of long aliphatic polyamides. This review is a reference with respect to the structure-properties relationship analysis for long aliphatic polymers.

In spite of the enthusiasm for ricinoleic acid and its derivatives, other fatty acids have been derivatized and employed as building blocks for polyamide preparation. Several reactions such as enzymatic 
oxygenation, metathesis or thiol-ene coupling were applied to fatty acids to access polymerizable building blocks.

Regarding first the design of bifunctional monomers, several research groups were interested in erucic acid obtained from the crambeseed oil in order to prepare PA-x,13 (Figure 75). Indeed, the brassylic acid (a C13 diacid) can be synthesized from the erucic acid cleavage and also derivatized in 1,13-tridecane diamine using specific conditions. ${ }^{440,441}$ PA-13,13 has been prepared from the polycondensation of both diamine and diacid from brassylic acid, resulting in polyamide with $\mathrm{Tg}$ of $50^{\circ} \mathrm{C}$ and $\mathrm{Tm}$ of $174^{\circ} \mathrm{C} .^{442} \mathrm{In}$ this line, PA-13,6 has been synthesized from 1,13-tridecane diamine and adipic acid ${ }^{443}$ and displayed similar Tg but a higher melting temperature of $206^{\circ} \mathrm{C}$ than PA-13,13. This feature was explained by crystallite reorganization in this material. ${ }^{444}$ More generally, the brassylic-based polyamides exhibited lower water uptake than PA-11 or PA-6,10.

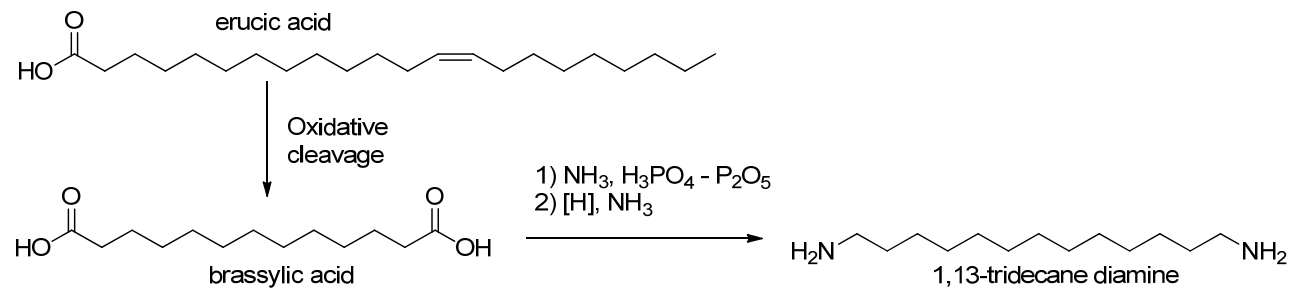

Figure 75. Synthesis of $\mathrm{C} 13$ dimers from erucic acid

Besides, a naturally occurring unsaturated $\mathrm{C} 18$ building block coming from the enzymatic oxygenation of oleic acid (using Cytrochrom P450 monooxygenase enzymes) ${ }^{445}$ and called Z-octadec-9-enedioic (Figure 76) has been copolymerized with several aliphatic and cyclo-aliphatic diamines. ${ }^{446}$ The molecular weights of the resulting polyamides were close to $18000 \mathrm{~g} . \mathrm{mol}^{-1}$ and displayed lower $\mathrm{Tg}$ and $\mathrm{Tm}$ than their corresponding saturated polyamides. ${ }^{447,448}$

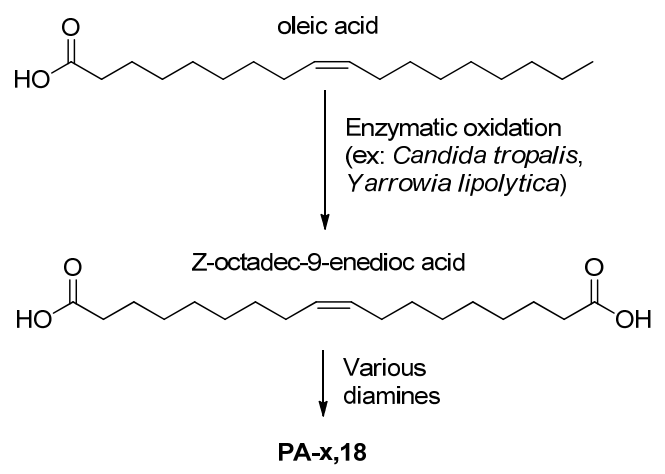

Figure 76. Z-octadec-9-enedioc acid from oleic acid.

Mecking and coll. ${ }^{449}$ reported the synthesis of linear saturated monomers containing 19 or 23 carbons on their aliphatic chains from respectively methyl oleate and methyl erucate following an isomerizing alkoxycarbonylation and a subsequent hydrolysis. Long aliphatic $\alpha, \omega$-diamines can be synthesized via a four-step reaction including diol and diazide intermediates further reduced in the corresponding diamines (Figure 77). Interestingly, Walther et al. ${ }^{450}$ also prepared the $\mathrm{C} 19$ diamine from the corresponding diol with an excess of ammonia at $140^{\circ} \mathrm{C}$ for $48 \mathrm{~h}$. Both diacids and diamines coming from methyl oleate and methyl erucate were polymerized to access novel long-chain PAs with molecular weights around 10000 g. $\mathrm{mol}^{-1}$. As expected, higher melting temperatures were observed for PA-11,23 and PA-12,23 than for PA-23,19 and PA-23,23. ${ }^{435}$ 


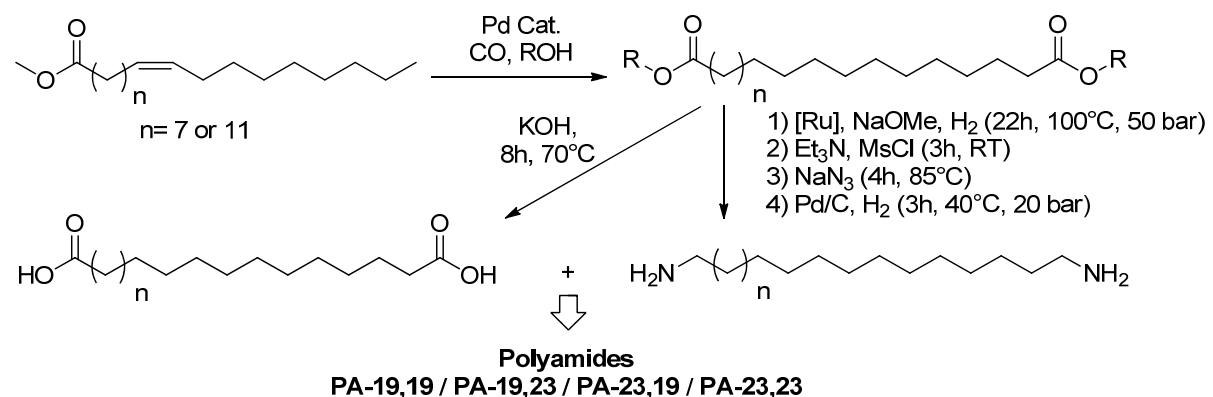

Figure 77. Diacids and diamines from methyl erucate and methyl oleate.

Furthermore, the preparation of unsaturated PA-x,20 have been carried out following two strategies. First, the monomer resulting from the self-metathesis of 10-undecenoic acid was polymerized with various diamines using TBD as catalyst (Figure 78). The second strategy involved the ADMET of the bisunsaturated diamide obtained via the dimerization of methyl 10-undecenoate under TBD catalysis. ${ }^{439,446}$ Both routes conducted to unsaturated aliphatic PA-x,20 with molecular weights in the range 5300-14700 g.mol ${ }^{-1}$ and Tm comprised in between 180 and $226^{\circ} \mathrm{C}$. Considering the presence of unsaturation within these PAs, ranges of application are restricted as the exposition to UV or air accelerates the material aging.

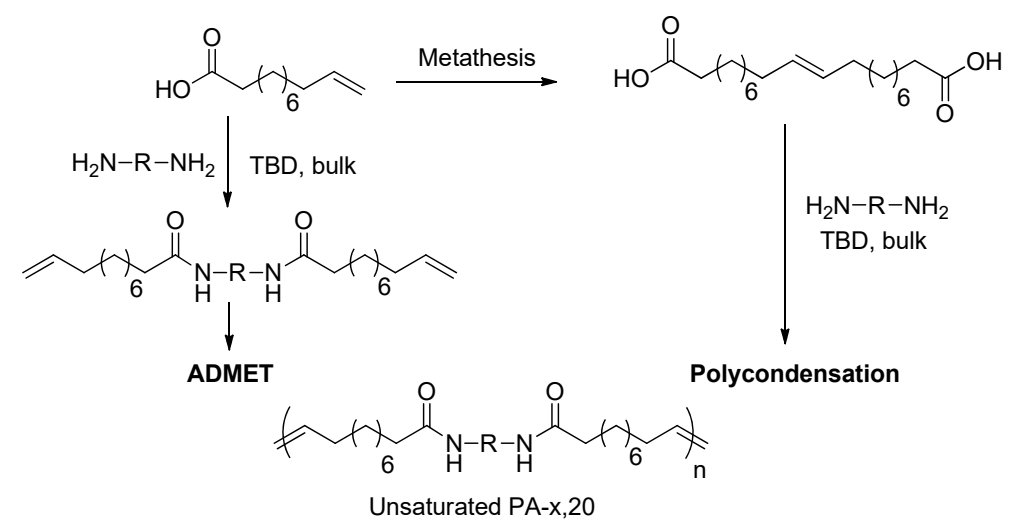

Figure 78. Preparation of fatty acid-based PA-x,20 via polycondensation or metathesis.

Interesting dimers originate from the dimerization between oleic and linoleic acids, well-known as Priplast ${ }^{\circledR}$ range commercialized by CRODA, were employed as comonomer for polyamide preparation. ${ }^{451,452}$ The long alkyl pendant chain imparted by this dimer conferred flexibility and lowered crystallinity to the resulting material in comparison with conventional polyamides, making them good candidates for printing inks and varnishes. ${ }^{453}$ Other C36-dimer fatty acids were synthesized by Meier and coworkers ${ }^{454}$ via the thiol-ene coupling of methyl oleate with ethane-1,2-dithiol under UV irradiation using DMPA as initiator. Copolyamides were prepared by polycondensation of hexamethylenediamine, dimethyl adipate and the dimer fatty acid and displayed lower water uptake than PA-6,6 due to the long alkyl chain conferring hydrophobicity.

With respect to the synthesis of AB-type monomers, interesting strategies and chemical transformations have been applied to fatty acids to design new PA-x polyamides. For instance, Meier and coll. ${ }^{455}$ performed the thiol-ene coupling of cysteamine hydrochloride with methyl undecenoate, methyl erucate and methyl oleate unsaturations to design AB-type monomers for sustainable polyamides (Figure 79). Tm values were tuned according to the monomer polymerized. Indeed, PA obtained from methyl 
undecenoate-based monomer displayed a Tm of $138{ }^{\circ} \mathrm{C}$ whereas the dangling chains of the methyl erucate-based one conferred to the final polyamide a $\mathrm{Tm}$ of $43^{\circ} \mathrm{C}$.

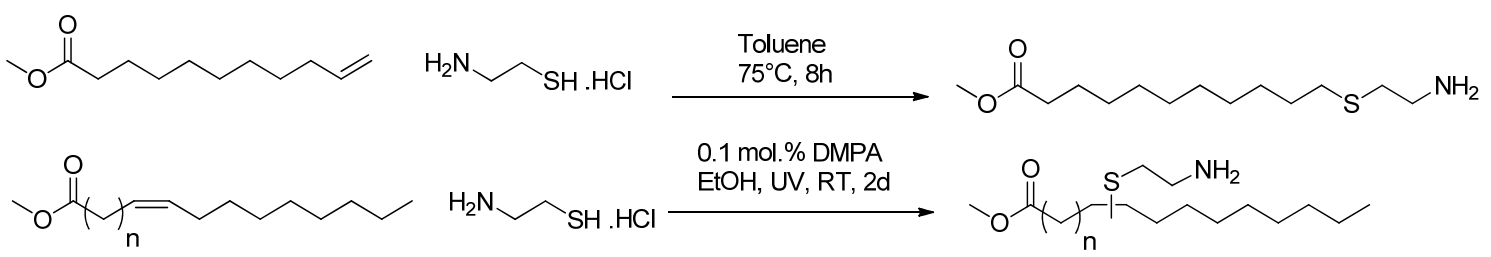

Figure 79. Thiol-ene reaction with cysteamine hydrochloride and fatty acid methyl esters to obtain AB selfcondensable monomers.

Amino-esters were also prepared by cross-metathesis of either acrylonitrile and fatty acid methyl esters (10-undecenoate, dimethyl octadec-9-ene-1,18-dioate and methyl ricinoleate) or 10-undecenenitrile with methyl acrylate. Both nitrile derivatives were reduced in their corresponding amine resulting in $\mathrm{AB}$ selfcondensable monomers for polyamide synthesis. ${ }^{456,457}$

Functionalization of methyl oleate and methyl erucate unsaturations via bromination, further reaction with $\mathrm{NaN}_{3}$ and subsequent hydrogenation was also carried out by Winckler and co-workers. ${ }^{458}$ PAs resulting from their self-polycondensation exhibited molecular weights in the range 6500-7900 g. $\mathrm{mol}^{-1}$ and Tm in between 40 and $89{ }^{\circ} \mathrm{C}$. A similar strategy involving oxofunctionalization of fatty acid methyl ester unsaturations was lately engaged by the same research group. ${ }^{459}$

Another atypical example of AB-type monomer is $\omega$-amino lauric acid obtained from palm kernel oil via a biotechnological route with the aim to prepare PA-12 by self-polycondensation. This new route could enable the replacement of the petroleum-based Vestamid® L (Evonik, PA-12).

Only one example of fatty acid-based PA produced via ROP has been reported in the literature. ${ }^{460}$ Indeed, the multi-step synthesis involved to obtain a polymerizable lactam is not viable at the industrial scale. Contrarily, terpenes and glucose were mentioned in a recent review ${ }^{429}$ to be good candidates for the preparation of sustainable lactams.

\section{Polyurethanes}

Linear thermoplastic polyurethanes (TPUs) are commonly used because of their excellent properties, such as good transparency, tunable stiffness, good wear resistance, excellent biocompatibility, etc. These properties are obtained thanks to the alternating of soft and hard segments in the structure of the polymer. Soft segments are usually flexible low $\mathrm{Tg}$ polyether or polyester chains with molecular weight between 1000 and 4000 g.mol ${ }^{-1}$ and can be easily obtained from fatty acid derivatives. Hard domains are usually crystalline with high melting points. The polyether polyols represent $75 \%$ of the petrochemical polyols used for PUs. Only one exemple of TPUs from polyether diol was reported from vegetable oil derivatives. ${ }^{461}$ This polyether diol containing ester pendant groups was obtained by the acid-catalyzed ring-opening polymerization of epoxidized methyl oleate and partial reduction with lithium aluminum hydride $\left(\mathrm{LiAlH}_{4}\right)$ (Figure 80). Polyols with various hydroxyl functionality degrees were obtained depending on the conversion of the reduction step. The TPU prepared from this polyether diol and MDI presented a low $\mathrm{Tg}$ of $-15^{\circ} \mathrm{C}$ and an interesting degradation temperature at $5 \%$ weight loss of $307^{\circ} \mathrm{C}$. 


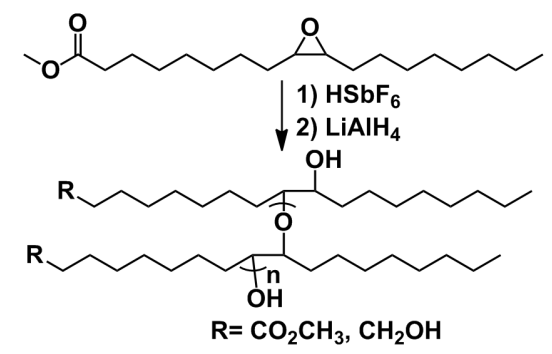

Figure 80. Polyether diol containing ester pendant groups obtained by the acid-catalyzed ring-opening polymerization of epoxidized methyl oleate and a partial reduction with lithium aluminum hydride.

The synthesis of diols containing ester group have been much more widely explored due to the initial presence of ester or acid function in vegetable oil derivatives. Polyester diols, diols containing ester and/or amide linkages, dimer diols, linear saturated or unsaturated diols and diols containing thioether linkages are referenced using fatty acids or fatty acid methyl esters as starting materials. ${ }^{462-468} \mathrm{Few}$ research groups have prepared low Tg polyester diols from vegetable oil derivatives to use them in segmented thermoplastic elastomer polyurethanes (TPUs). Petrovic and coll. have used a poly(ricinoleic acid) diol as soft segment to prepare a series of TPUs by reacting MDI and 1,4-butanediol with various soft segment weight concentrations ranging from $40 \%$ to $70 \%$ (Figure 81 ). Such TPUs displayed a microphase separation and two glass transition temperatures at around $-50^{\circ} \mathrm{C}$ and $100^{\circ} \mathrm{C}$. The same group has studied the morphology of these TPUs by DMA, AFM and USAXS. ${ }^{463}$ A co-continuous morphology with domains around $15 \mathrm{~nm}$ was obtained in the case of the TPUs with $50 \%$ of soft segment weight concentrations. However, in the case of TPUs with $70 \mathrm{wt} \%$ of soft segment, dispersed hard domains were observed in the soft matrix. The biodegradation of these TPUs has been investigated by respirometry. ${ }^{467}$ These TPUs with poly(ricinoleic acid) (PRic) as soft segments have been found to exhibit faster degradation rates than the corresponding petrochemical poly(ester urethane)s, even if this rate is relatively slow (about $11 \%$ carbon after 30 days). The authors observed that TPUs with a co-continuous morphology demonstrated a slightly slower biodegradation than those with dispersed hard domains in the soft phase.

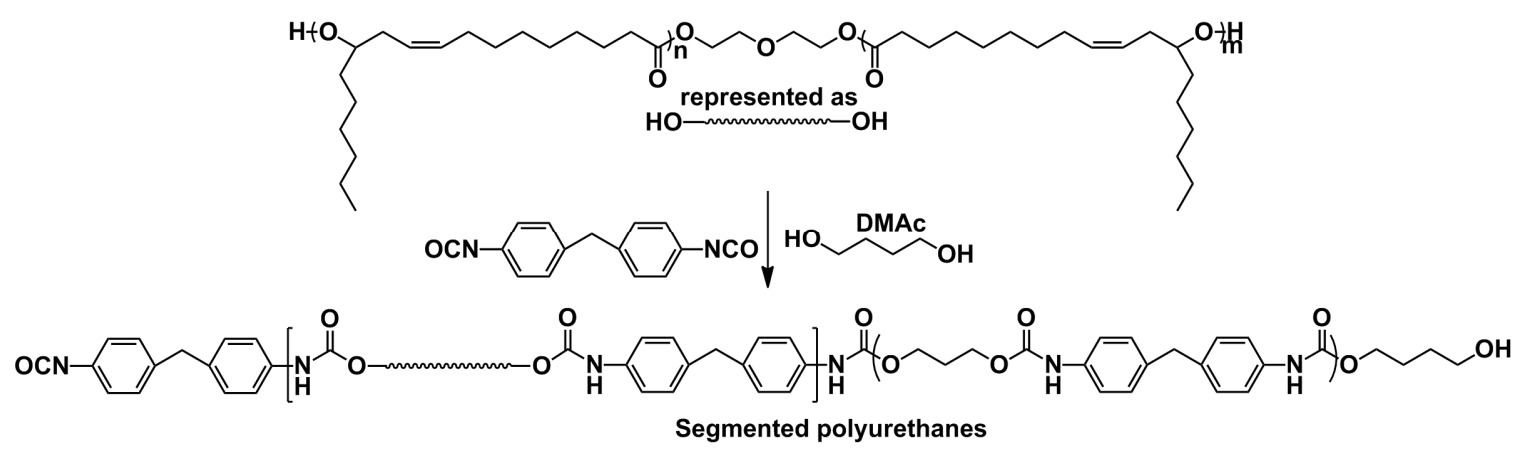

Figure 81. Thermoplastic elastomer polyurethane prepared from poly(ricinoleic acid).

An alternative to synthesize TPUs with a fatty acid based diol as soft segment, is to use the CRODA polyester diol synthesized by transesterification between dimerized fatty acids (obtained by Diels-Alder reaction) and an excess of linear diol. ${ }^{469,470}$ By selecting the appropriate hard segment concentration, different thermo-mechanical properties were achieved and could fulfill some industrial requirements in diverse fields.

In order to obtain well-defined monomers, several research groups have synthesized accurate bisunsaturated compounds with one or two ester functions from methyl oleate and methyl undecenoate or from the corresponding acid derivatives. ${ }^{471-474}$ Afterwards, the hydroxyl moieties were introduced on the 
double bonds by thiol-ene reaction or epoxy ring-opening (Figure 82). By using the thiol-ene reaction method, asymmetric ester diols have been prepared from oleic and undecylenic acids by esterification with allyl alcohol and thiol-ene reaction with 2-mercaptoethanol. Polymerizations with MDI in the presence of tin (II) 2-ethylhexanoate as catalyst, led to TPUs with molecular weights from 50 to 70 kg. $\mathrm{mol}^{-1}$ and dispersity in the range 1.6-1.9. Amorphous $\left(\mathrm{Tg}=8^{\circ} \mathrm{C}\right.$ to $\left.20^{\circ} \mathrm{C}\right)$ to semi-crystalline PUs $\left(\mathrm{Tm}=124^{\circ} \mathrm{C}\right)$ were obtained depending on the fatty derivative used. These TPUs revealed both good thermal and mechanical properties as well as no cytotoxic response thanks to MTT test, which make them possible candidates for biomedical purposes.

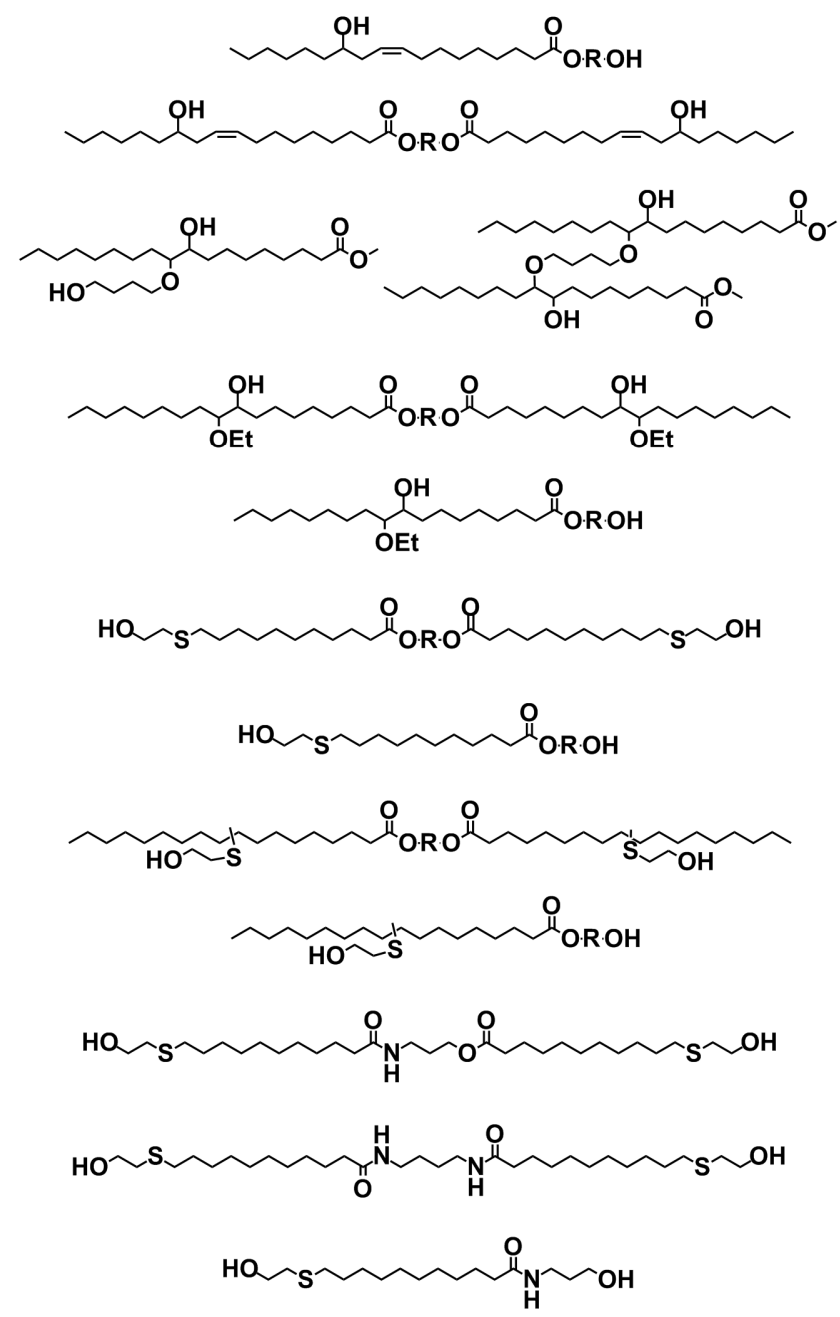

Figure 82. Range of diols (PU precursors) that can be obtained from methyl undecenoate, methyl oleate and methyl ricinoleate by transesterification, amidation, thiol-ene reaction and epoxidation/ring-opening of the epoxide

Cramail and coll. also prepared sugar-based fatty ester polyols by selective transesterification of epoxidized methyl or ethyl oleate with unprotected methyl $\alpha$-D-glucopyranoside and sucrose respectively, followed by hydrolysis of the epoxide moiety. ${ }^{475}$ The polyols were then used in polyurethanes with IPDI in the presence of dibutyl tin dilaurate (DBTDL) as a catalyst. The authors noticed that the reactivity of the hydroxyl functions attached to the sugar and to the fatty ester chain moieties respectively could be discriminated with respect to the solvent used, enabling the synthesis of either linear or cross-linked PUs.

Vegetable oil based poly- or di-isocyanates have effectively been studied in a much less extent perhaps as a consequence of the inherent aliphatic structure of the isocyanate that could be prepared. Indeed, the most industrially used diisocyanates are aromatic namely MDI and TDI due to a higher reactivity required to produce foams. Thus, the use of aliphatic diisocyanates is limited to coatings in which the absence of 
unsaturation is profitable. In industry, isocyanates are synthesized from primary amines by phosgenation. As primary amines are not easily introduced into vegetable oils, other strategies have been developed on vegetable oils and their derivatives. For instance, isocyanates can be synthesized by taking the advantage of the Curtius, Hoffman and Lossen rearrangements, which involve nitrene intermediates. Narine and coll. used the Curtius rearrangement to prepare oleic acid-based linear diisocyanates, both saturated and unsaturated. ${ }^{476,477}$ Two linear diacids were first synthesized then converted to acyl azides by the reaction with sodium azide and, upon heating, had decomposed to isocyanates. Cramail and coll. also reported the synthesis of fatty acid based diisocyanates using the Curtius rearrangement through acyl hydrazide fatty acid based derivatives without the use of harmful sodium azide (Figure 83). ${ }^{478}$ Diesters were first synthesized and then reacted with hydrazine hydrate to form diacyl hydrazides in quantitative yields. Afterwards, these diacyl hydrazides were converted into diacyl azide then into diisocyanate via the Curtius rearrangement. A series of partially and fully vegetable oil based TPUs were synthesized and a large range of thermo-mechanical properties were achieved. Relatively good thermal behaviors were observed with decomposition temperatures at $5 \%$ weight loss from $230^{\circ} \mathrm{C}$ to $280^{\circ} \mathrm{C}$. For some polyurethanes, a close resemblance to HDPE was obtained in terms of solubility and thermal transitions with melting points close to $145^{\circ} \mathrm{C}$

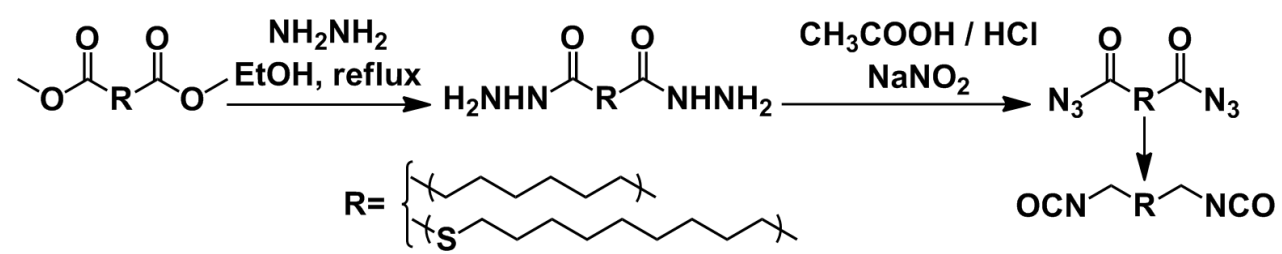

Figure 83. Fatty acid-based diisocyanates using hydrazine hydrate.

There are few examples in the literature of fatty acid-based polyurethanes obtained by transurethanization polycondensation. The monomers, bis-alkylcarbamates and bis-hydroxyalkylcarbamates, can be obtained from dimethylcarbonate or ethylene carbonate. Fatty acids can also be modified by other means to get methylcarbamate functionalities.

Narayan and coll. synthesized a bis-hydroxycarbamate using a dimerized fatty acid by transamidation of ethylene diamine on the carboxylic acid functions and further reaction with ethylene carbonate (Figure 84). The polymerization strategy is a self-polymerization of the bis-hydroxycarbamate. The polymers obtained at $150^{\circ} \mathrm{C}$ under reduced pressure exhibited a $\bar{M}_{\mathrm{n}}$ of $7.7 \mathrm{~kg} \cdot \mathrm{mol}^{-1}$ and $\bar{M}_{\mathrm{w}}$ of $14 \mathrm{~kg} \cdot \mathrm{mol}^{-1}$, a Tg of $10^{\circ} \mathrm{C}$ and a $\mathrm{Tm}$ of $73^{\circ} \mathrm{C}$.

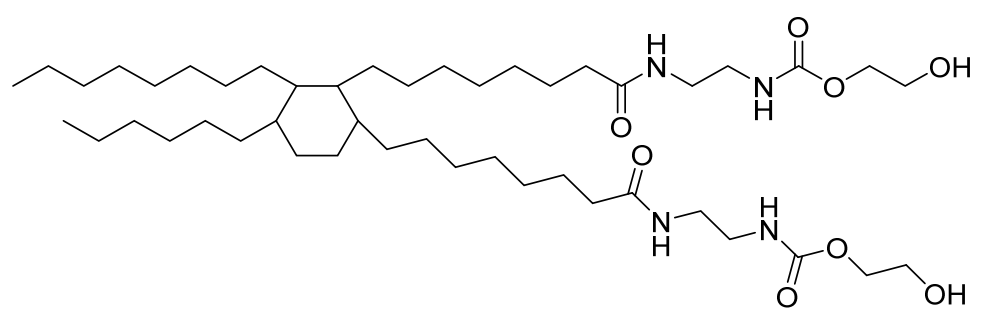

Figure 84. Fatty acid based bis-hydroxycarbamate

Burel and coll. synthesized various bio-based PUs from bio-based diols and bis-methylcarbamates. ${ }^{479}$ The bis-methylcarbamates were synthesized from bio-based diamines and DMC in the presence of TBD. The bis-methylcarbamates produced are presented Figure 85; the latter are derived from a fatty acid dimer and ricinoleic acid. The PUs were then synthesized by polycondensation with diols with TBD or $\mathrm{K}_{2} \mathrm{CO}_{3}$ as catalyst. $\mathrm{Tg}$ of the so-formed PUs are ranging from -38 to $-19^{\circ} \mathrm{C}$. Most PUs are amorphous due to the 
presence of dangling chains brought by the fatty acid derivatives, but some present a fusion temperature around $61-64^{\circ} \mathrm{C}$.

Bis-methylcarbamates
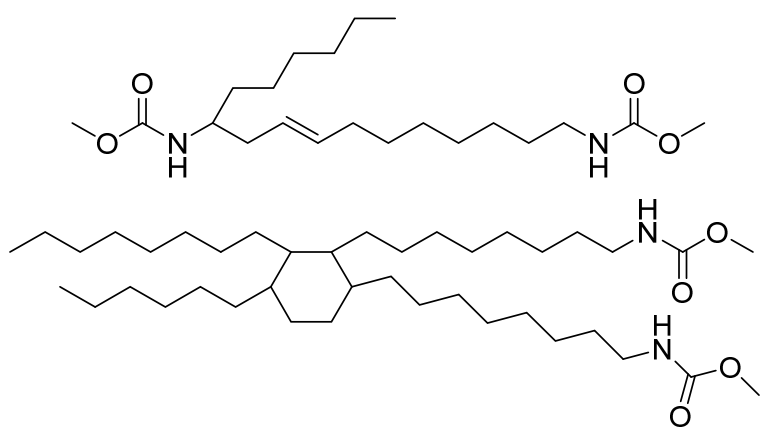

Diols
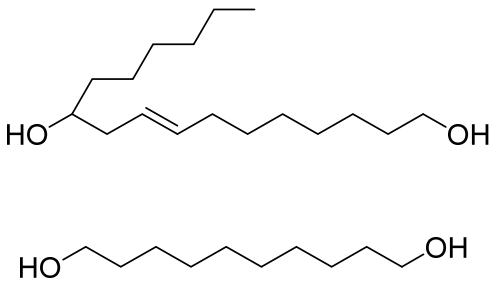

Figure 85. Monomers derived from fatty acids for the synthesis of PUs

The Lossen rearrangement is usually used to transform hydroxamic acids into isocyanates. Meier and coll. introduced a modified method to get the corresponding carbamates with a catalytic amount of an organic base such as TBD in the presence of methanol. ${ }^{480,481}$ Fatty acid methyl esters were easily turned into hydroxamic acids using hydroxylamine hydrochloride and potassium hydroxide. Bismethylcarbamates and fatty carbamates were synthesized using this method (Figure 86).

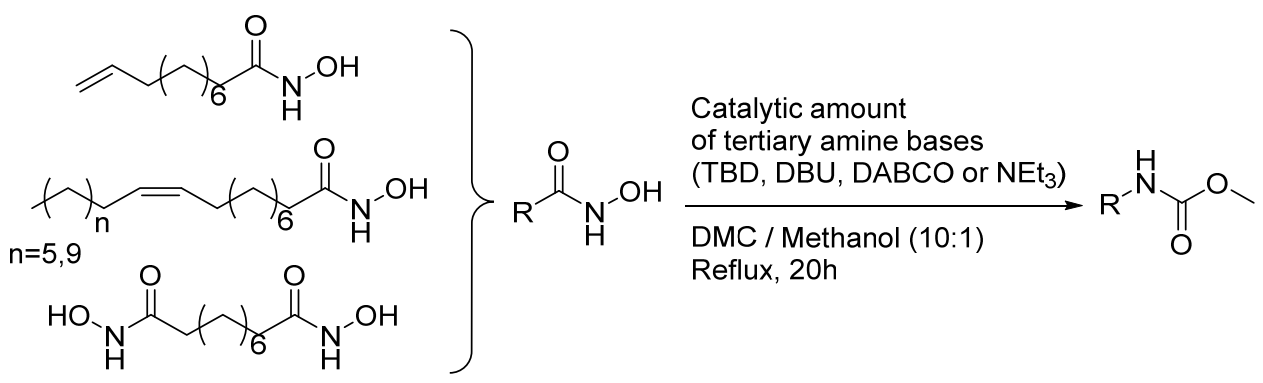

Figure 86. Catalytic Lossen rearrangement towards fatty acid-based carbamates

The polymerization of such bis-methylcarbamate with bio-based aliphatic diols was performed in bulk with catalytic amount of TBD under reduced pressure by increasing the temperature from $120^{\circ} \mathrm{C}$ to $160^{\circ} \mathrm{C}$. The PUs obtained were crystalline with melting temperatures ranging from 120 to $145^{\circ} \mathrm{C}$. The molecular weights were comprised between $\bar{M}_{\mathrm{n}}=7.1$ and $24.6 \mathrm{~kg} \cdot \mathrm{mol}^{-1}$ with dispersities of 1.2 to 2.1 .

AB-type synthons were synthesized by Cramail and coll. (Figure 87). The hydroxyl function is naturally occurring in the alkyl chain of ricinoleic acid or it was brought by thiol-ene addition on the double bond of oleic acid and derivatives. The methylcarbamate functionality was brought by transforming the carboxylic acid into acyl azide moiety. The acyl azide was then reacted with methanol to form the methylcarbamate. ${ }^{482,483}$ Self-polymerizations were performed in the presence of $\mathrm{Ti}(\mathrm{OBu})_{4}$ as catalyst at $130^{\circ} \mathrm{C}$ under $\mathrm{N}_{2}$ aTmosphere for $4 \mathrm{~h}$ and then under reduced pressure for $2 \mathrm{~h}$. PUs obtained had low molecular weights with $\bar{M}_{\mathrm{n}}$ from 2.1 to $6.9 \mathrm{~kg} \cdot \mathrm{mol}^{-1}$. Tgs were ranging from -44 to $-18^{\circ} \mathrm{C}$. A second glass transition temperature was visible at around 25 to $32^{\circ} \mathrm{C}$ for PUs synthesized from thiol-ene modified synthons, which indicated a phase-separated morphology. 


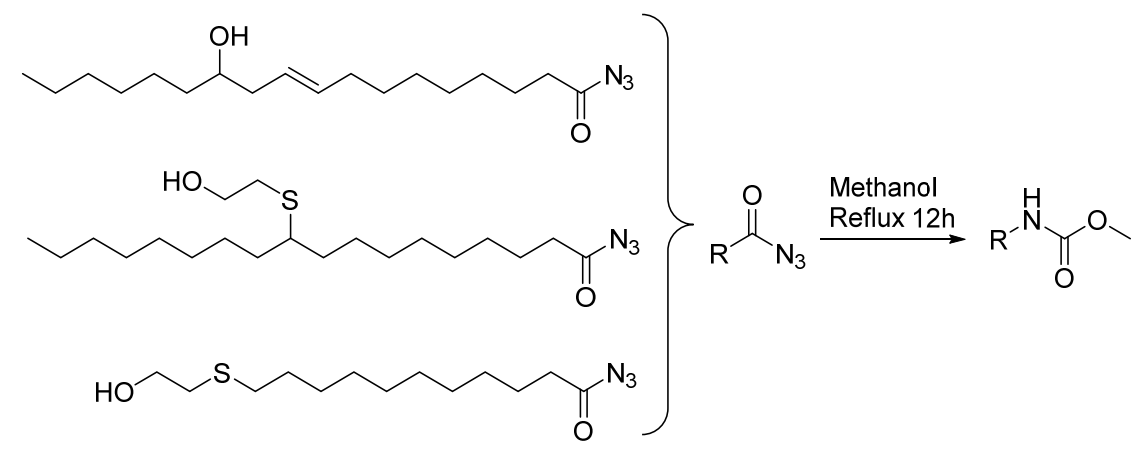

Figure 87. Fatty acids-based AB-type synthons

Among non-isocyanate and non-phosgene routes to PUs, the approach involving the reaction of cyclic carbonate and amine functions is one of the most studied. The main route is the polyaddition of bis-5membered cyclic carbonates and diamines leading to the formation of linear PHUs with primary or secondary alcohols, as already illustrated in Figure 70.

As far as vegetable oils are concerned, the 5-membered cyclic carbonate monomers have been mainly synthesized from glycerol carbonate or by epoxidation followed by carbonation of the fatty acid chain unsaturations.

The first example relating the synthesis of thermoplastic PHUs from fatty acid derivatives was reported by Boyer et al. ${ }^{484}$ The fatty acid-based bis 5 -membered cyclic carbonates were synthesized by a three-step process composed of (1) a transesterification of fatty acid methyl esters, (2) an epoxidation of the double bonds and (3) a carbonation of the resulting epoxides. The solubility of the fatty acid or triglyceride-based mono-, bis- and poly-epoxides in supercritical $\mathrm{CO}_{2}$ has been studied. ${ }^{485}$ Two bis 5 -membered cyclic carbonates, prepared from methyl oleate and methyl undecenoate respectively, were polymerized with ethane-1,2-diamine (EDA) and isophorone diamine (IPDA) to form PHUs containing hydroxyl moieties (Figure 88). ${ }^{484}$ The PHUs exhibited molecular weights up to $13500 \mathrm{~g} \cdot \mathrm{mol}^{-1}\left(\bar{M}_{\mathrm{w}}\right)$ and relatively low Tgs ranging from $-25^{\circ} \mathrm{C}$ to $-13^{\circ} \mathrm{C}$. Nevertheless, an amidation side reaction occurred between EDA and the ester linkages of the diester bis-cyclic carbonates, giving amide groups which can partly explain the low molecular weights obtained.

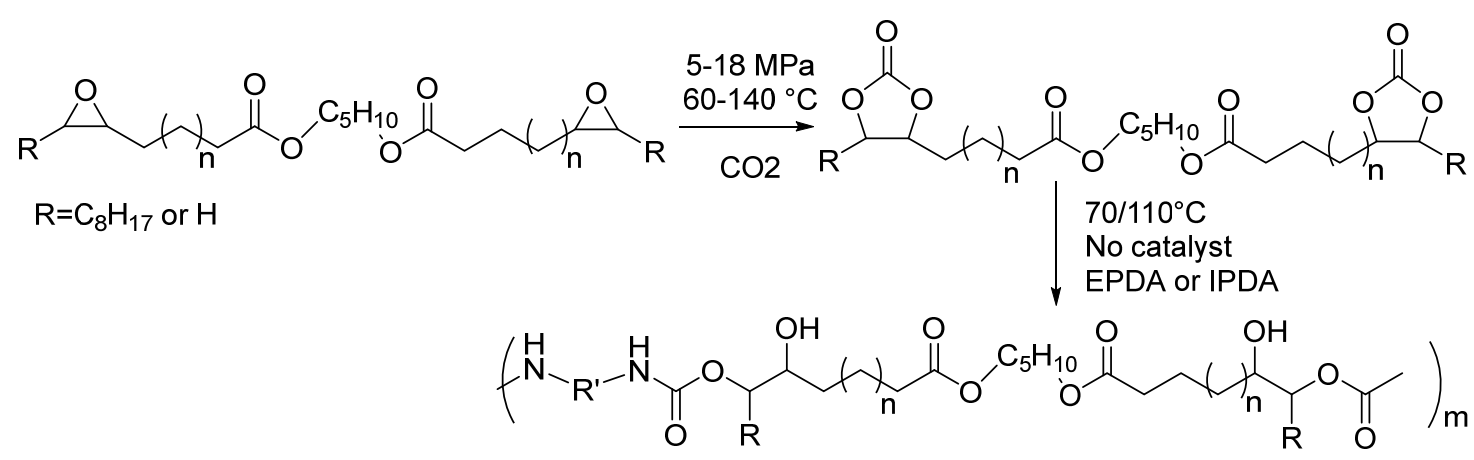

Figure 88. Cyclic carbonates and poly(hydroxyurethane)s from fatty acid or fatty acid methyl ester.

We further reported the synthesis of several fatty acid based bisCC using the epoxidation/carbonation strategy. ${ }^{486}$ Two fatty acid chains were first dimerized by amidation or transesterification reactions with butane-1,4-diamine, piperazine, N,N'-dimethylpropane-1,3-diamine, N,N'-dihexyldecane-1,10-diamine and 1,3-propanediol. The produced aliphatic bis-unsaturated substrates were epoxidized and carbonated following the procedure indicated in Figure 89. The polymerization of these dimers presenting different 
central blocks with various diamines (such as Jeffamine, isophorone diamine or diaminobutane) led to PHUs exhibiting a broad range of $\mathrm{Tg}$ values from $-29^{\circ} \mathrm{C}$ to $55^{\circ} \mathrm{C}$.

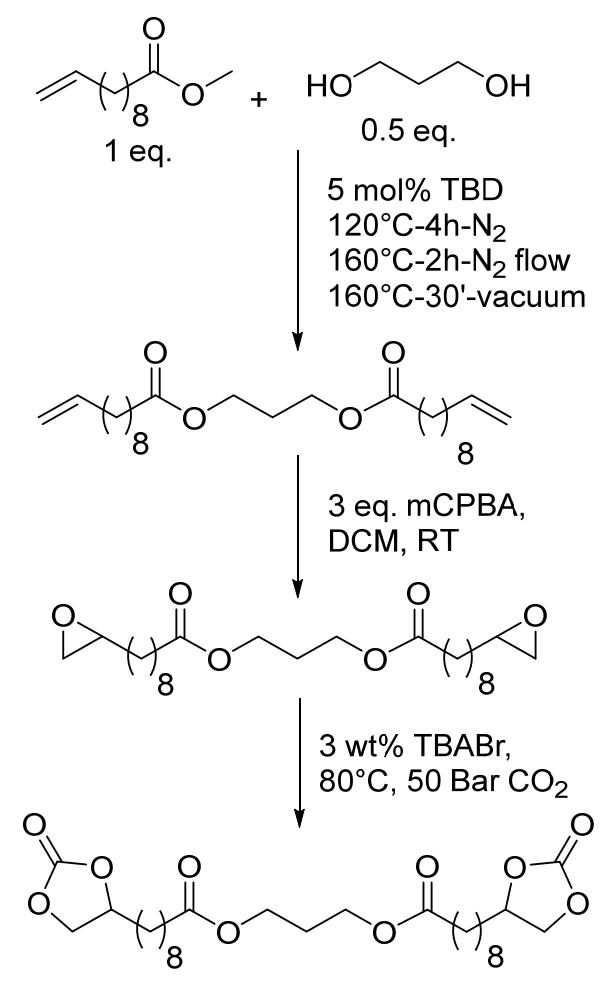

Figure 89. Strategy for the synthesis of 5-membered bis-cyclic carbonates from fatty acid derivatives.

In order to increase the reactivity of 5CC towards aminolysis, an alternative route consists in inserting a heteroatom nearby the cyclic carbonate to improve/activate its reactivity. ${ }^{487-496}$ Activated 5-membered cyclic carbonates were prepared from glycerol, thioglycerol and fatty acid derivatives (Figure 90). Ester, ether and sulfur moieties were introduced in $\beta$ position to the cyclic carbonate, in order to enhance its reactivity towards amines. ${ }^{497,498}{ }^{1} \mathrm{H}$ NMR kinetic investigation of the aminolysis of these cyclic carbonates demonstrated a higher reactivity compared to the one of alkyl substituted cyclic carbonates. In the case of ester-activated carbonates, a reactivity similar to the one of 6-membered ring cyclic carbonate was observed. Moreover, these carbonates exhibited amidation side-reactions with amines that could be however prevented by decreasing the temperature to room temperature. Poly(hydroxyurethane)s (PHUs) were then synthesized from these activated 5-membered ring cyclic carbonates at $70^{\circ} \mathrm{C}$ in $\mathrm{DMF}$ and exhibited molecular weights up to 13700 g.mol ${ }^{-1}$ with $\mathrm{Tg}$ in the range -26 to $-10^{\circ} \mathrm{C}$. 


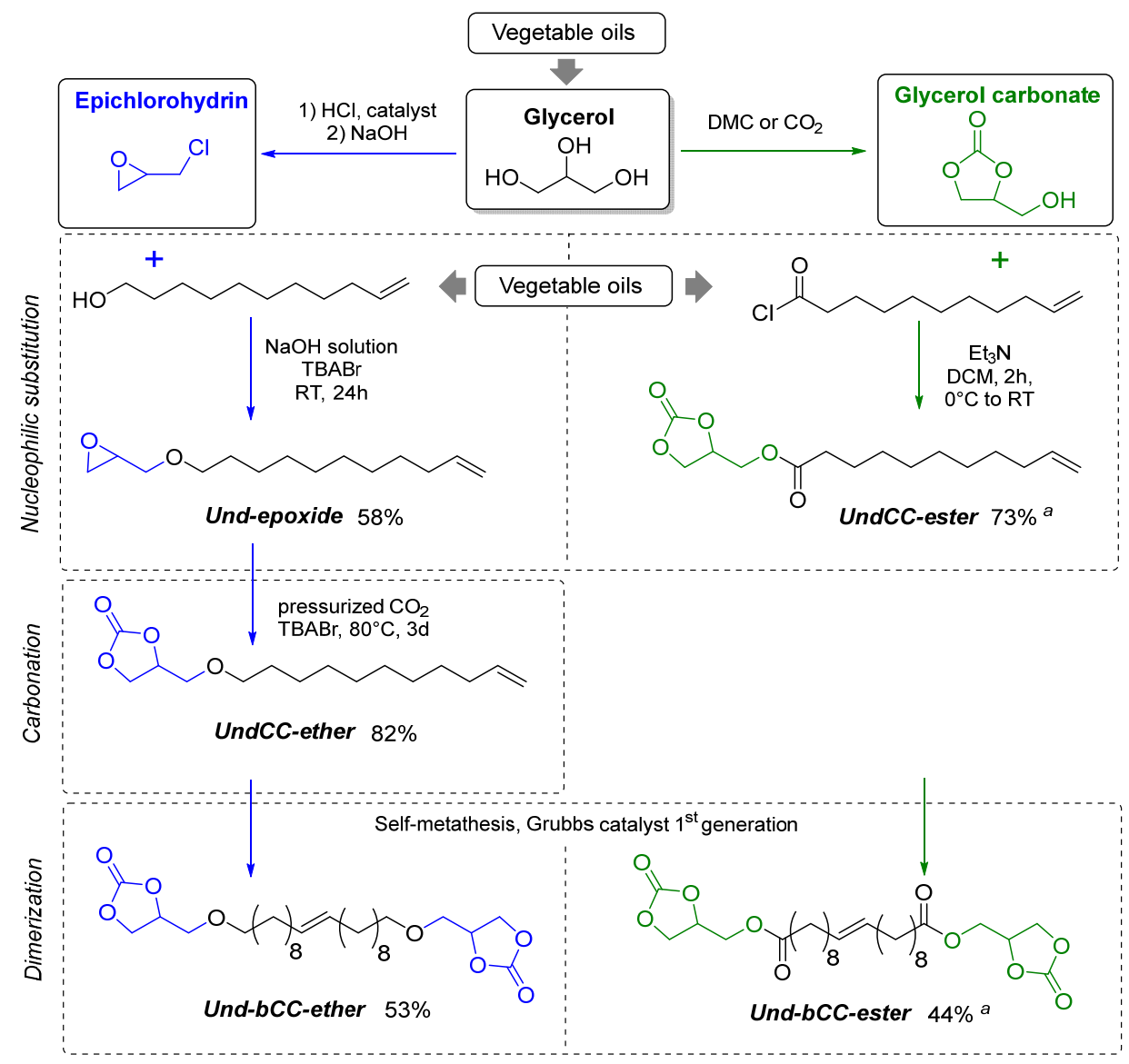

Figure 90. Strategy for the synthesis of 'activated' 5-membered bis-cyclic carbonates from fatty acid derivatives

Another strategy developed by Maisonneuve et al. ${ }^{499}$ focused on the design of more strained and reactive 6-membered cyclic carbonates. The latter were synthesized from methyl undecenoate by malonization, reduction and carbonation of the resulting diol. The mono-cyclic carbonate obtained was dimerized either by thiol-ene or metathesis reactions (Figure 91). The synthesized bifunctional 6-membered cyclic carbonates were effectively used as building blocks for thermoplastic isocyanate-free PHUs in combination with dodecane-1,12-diamine as comonomer. Molecular weights up to $23000 \mathrm{~g} \cdot \mathrm{mol}^{-1}$ $(Đ=1.7)$ were obtained after only one day in $\mathrm{DMF}$ at $50^{\circ} \mathrm{C}$. 


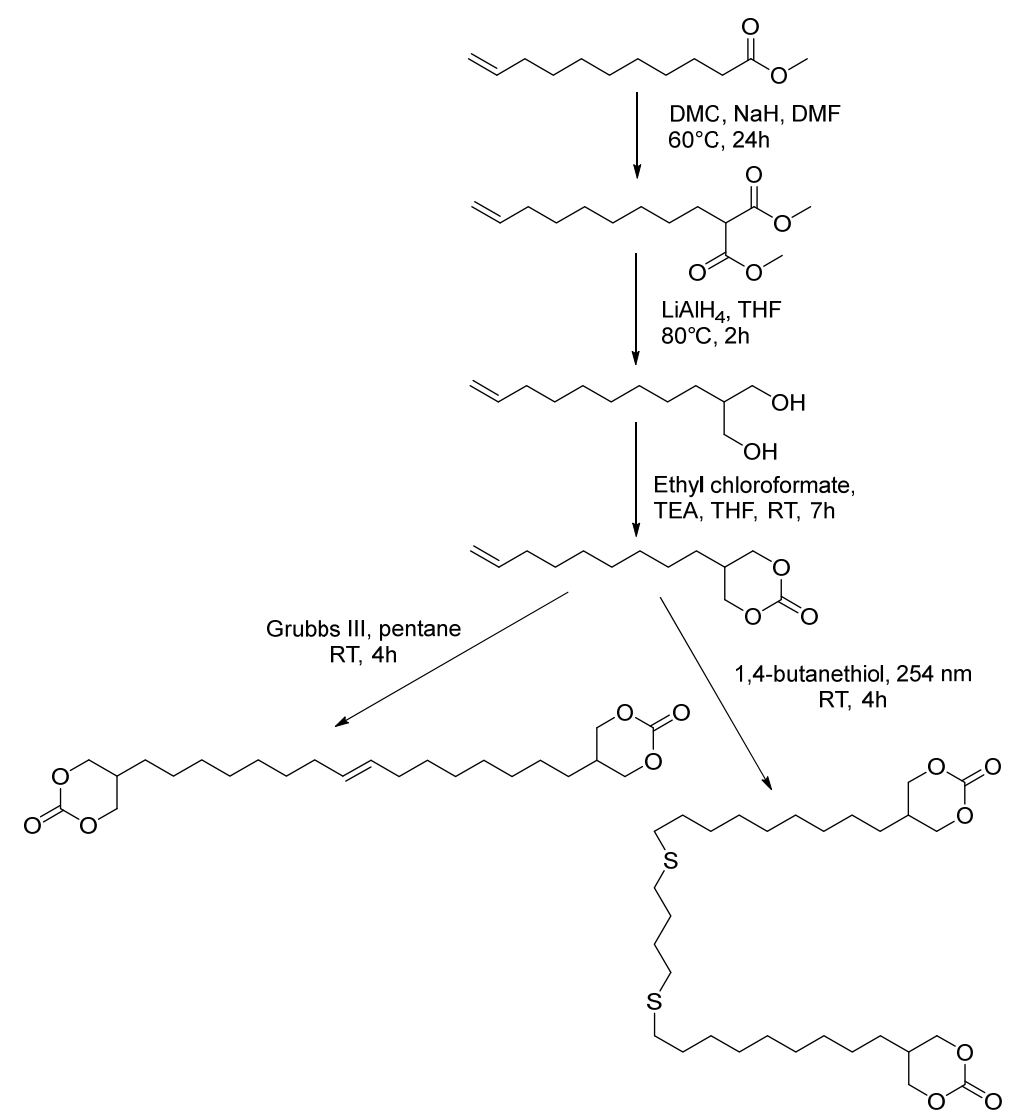

Figure 91. Procedure for bis 6-membered cyclic carbonate synthesis from methyl undecenoate.

As a conclusion of this part, activated 5-CCs and 6-CCs have a comparable reactivity with respect to aminolysis. However, 5-CCs seem to be more relevant in terms of synthesis at the industrial scale. Hence, fatty acid derivatives represent a platform of bio-based building blocks for poly(hydroxyurethane) synthesis. Caillol and coll. ${ }^{500}$ highlighted the large possibilities offered by this platform for both linear and cross-linked polymers. The abundance of reactive hydroxyl functionalities present along the PHU backbone could enable further curing process and post-functionalization. ${ }^{496}$

\section{Polyesters}

As already discussed, polyesters (PEs) combine unique properties such as film forming ability, potential biodegradability and biocompatibility. ${ }^{501}$ Indeed, under specific conditions, PEs are prone to be degraded via reactions onto ester linkages, known to be hydrolysable. Depending on the monomers and on the polymerization methods, a wide range of applications is covered. Indeed, polyesters can be employed in fibers, rubbers, plasticizers, surface coatings, or resins, ${ }^{502,503}$ demonstrating the high potential and versatility of such materials. Polyesters are commonly prepared from the polycondensation by esterification between diols and diacids, or by transesterification between diols and diesters, resulting in the production of water or methanol as by product (Figure 92-(1)). However, the transesterification pathways require high temperature and catalysts ${ }^{426,504}$ for the preparation of high molecular weight PEs. Another strategy is to employ 'activated' monomers such as acid chlorides or anhydrides to circumvent this issue. As $\mathrm{A}_{2}$ - $\mathrm{B}_{2}$-type polymerization requires an accurate stoichiometry (1:1) between both monomers, another strategy considering the polycondensation of $\omega$-hydroxy acids (AB-type monomers) has been considered. Ring opening polymerization of lactones and ADMET polymerization of estercontaining dienes were also developed for the preparation of polyesters (Figure 92-(2) and (3)). 
(1) Polycondensation Diacid + Diol

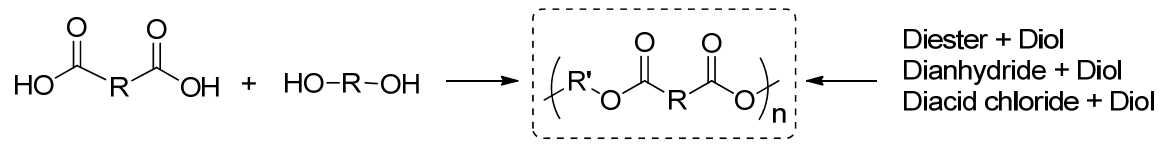

(2) Self-polycondensation<smiles>[R]OC(=O)O</smiles>

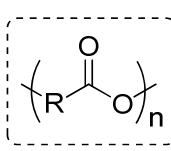

(3) Ring-opening polymerization

\section{Lactone \\ $\longleftarrow$

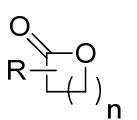

(4) ADMET Polymerization

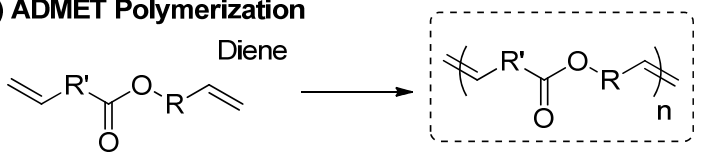

Figure 92. Different routes towards polyesters preparation

Many reviews reported the possibilities offered to prepare bio-based polyesters from sugars, fatty acids or terpenes from which carboxylic acids are easily obtained. ${ }^{404,192,505,506}$ Due to the extensive number of studies covering the subject, the scope of this sub-chapter will only focus on the most relevant pure fatty acid-based polyesters and their relative properties.

Fatty acids represent a promising feedstock for polyester synthesis as they provide ester, acid and sometimes hydroxyl functions. Additionally, fatty acids lead to linear aliphatic polyesters known to be degradable in a reasonable time scale, making them good candidates for drug delivery systems. ${ }^{507}$ Fatty acid based-polyesters can also mimic polyethylene while presenting original features such as biodegradability and polarity.

Again, ricinoleic acid derived from castor oil is an interesting starting material for the synthesis of ABand $\mathrm{A}_{2}$-type monomers for renewable polyesters as reported by Kunduru et al. ${ }^{508}$ (Figure 93) This review highlights the biocompatibility of such starting materials making them suitable for biomedical applications. The carboxylic acid and the naturally occurring hydroxyl function present on ricinoleic acid enable its self-polycondensation to yield poly(ricinoleate) also named PRic. Enzymatic polytransesterification of ricinoleic acid has been carried out using several lipases and enabled to yield polyesters with molecular weights comprised between 2000 and 100000 g.mol ${ }^{-1}$. ${ }^{209}$ The dangling fatty acid chains conferred particular properties to the resulting polyesters as a $\mathrm{Tg}$ of $-75^{\circ} \mathrm{C}$ was obtained for the highest molecular weight polyester. Extreme temperature above $200^{\circ} \mathrm{C}$ and tributyltin catalysts could also permit to prepare PRic. ${ }^{510}$ Ricinoleic acid can also be copolymerized with polyols in order to increase molecular weights and to expand the PRic range. ${ }^{511}$ Taking advantage of the unsaturations within PRics, the latter could be cross-linked/vulcanized to yield thermosetting elastomers. ${ }^{509,512}$

10-hydroxydecanoic acid as well as sebacic acid can be obtained from alkali fusion leading to polymerizable molecule through an $\mathrm{AB}$-type or an $\mathrm{A}_{2}-\mathrm{B}_{2}$-type process. These two molecules can also be reduced in their corresponding diol to yield another interesting $\mathrm{A}_{2}$ monomer ${ }^{513}$ Regarding sebacic acid, this castor oil-based monomer has already been incorporated in copolyesters applied as biodegradable delivery systems for biomedical applications. ${ }^{514,515}$ In a more original way, 9-hydroxynonanoic acid can be obtained from ozonolysis, reduction, transesterification and catalysis with $\mathrm{Ti}(\mathrm{iOPr})_{4}{ }^{516}$ and subsequently self-polymerized to yield a high molecular weight polymer $\left(62000 \mathrm{~g}^{\mathrm{mol}}{ }^{-1}\right)$ with higher $\mathrm{Tg}$, Tm and thermal stability than poly( $\varepsilon$-caprolactone), PCL. 


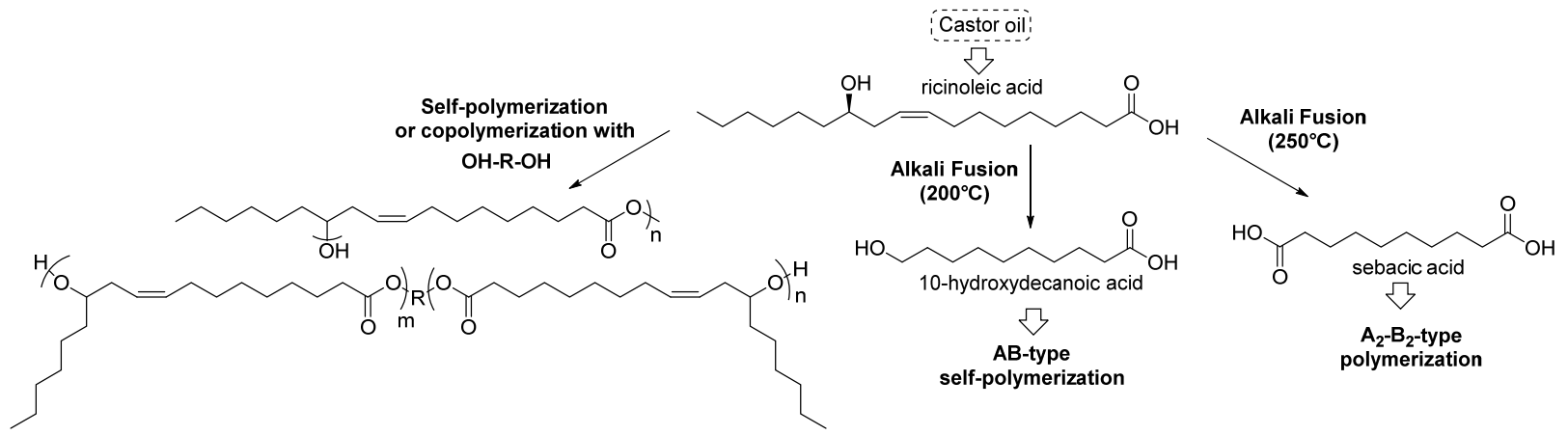

Figure 93. Castor oil as a platform for bio-based polyesters

Regarding other fatty acid-based condensable monomers, some of them were prepared from more original techniques. As an example, Cramail and coll. ${ }^{517}$ published the synthesis of hyperbranched polyesters from catalyzed self-polycondensation of $\mathrm{AB}_{\mathrm{n}}$-type monomer prepared from the ring-opening of epoxidized fatty acid methyl esters in acidic conditions (Figure 94). The prepared materials exhibited molecular weights in the range 3000-10000 g.mol ${ }^{-1}$ with dispersities comprised between 2 and 15 .

Another example describes the biochemical conversion of unsaturated fatty acids into $\alpha, \omega$-dicarboxylic acids by enzymatic oxidation of terminal methyl groups into a carboxylic function using Candida tropicalis. ${ }^{518}$ The unsaturated polyesters prepared via an enzymatic polycondensation of the prepared monomers with $\alpha, \omega$-diols displayed molecular weights in the range 25000-57000 g. $\mathrm{mol}^{-1}$ with relatively low $\operatorname{Tm}\left(23-40^{\circ} \mathrm{C}\right)$. Notably, these materials can also be post-functionalized or cross-linked through unsaturations within the backbone.

Besides, an original method to produce $\mathrm{A}_{2}$-type monomers was investigated few years ago by Mecking's group ${ }^{506}$ and consisted in the isomerizing carbonylation of unsaturated fatty acids with carbon monoxide using specific Pd catalysis. Dicarboxylic acids and diols containing 19 and 23 carbons were thus prepared and polymerized to achieve long aliphatic semi-crystalline polyesters with high Tm values between 86 and $100^{\circ} \mathrm{C}$.

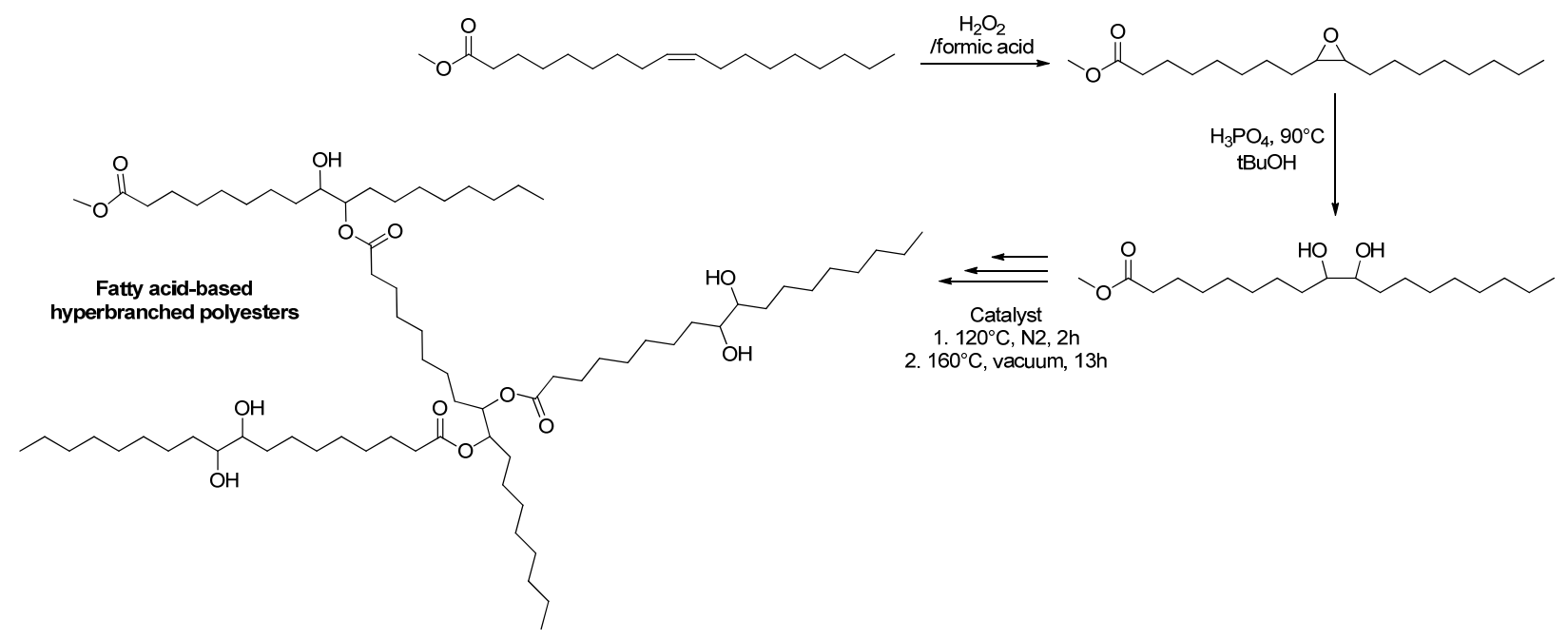

Figure 94. ABn-type monomer synthesis towards hyperbranched polyesters

Furthermore, ADMET and thiol-ene click chemistry have been extensively investigated for the design of $\mathrm{AB}$-type, $\mathrm{A}_{2}$-type and $\mathrm{AB}_{2}$-type monomers as well as polymerization techniques. For instance, the group of Meier ${ }^{519}$ carried out the preparation of different original fatty acid-based monomers from methyl castor oil derivatives (10-undecenoate, 10-undecenol) and 1,4-butanedithiol, methylthioglycolate, thioglycerol or mercaptoethanol via thiol-ene reactions (Figure 95). Depending on the thiol substrate used, $\mathrm{AB}-, \mathrm{A}_{2^{-}}$, or $\mathrm{AB}_{2}$-type monomers were prepared by bringing either ester or hydroxyl functions. 
After polycondensation, the resulting polymers displayed melting points in the range $50-71{ }^{\circ} \mathrm{C}$ and thermal stability up to $300^{\circ} \mathrm{C}$.

Following a similar strategy, Lebarbé et al ${ }^{520}$ synthesized symmetric and asymmetric fatty acid-based diols by associating transamidation (or transesterification) reactions and thiol-ene coupling of mercaptoethanol with fatty acid unsaturations (Figure 95). The original condensable monomers were polymerized with a diester synthesized from the self-metathesis of methyl 10-undecenoate. The resulting poly(ester-amide)s showed melting temperatures up to $127^{\circ} \mathrm{C}$ due to the presence of amide linkages.

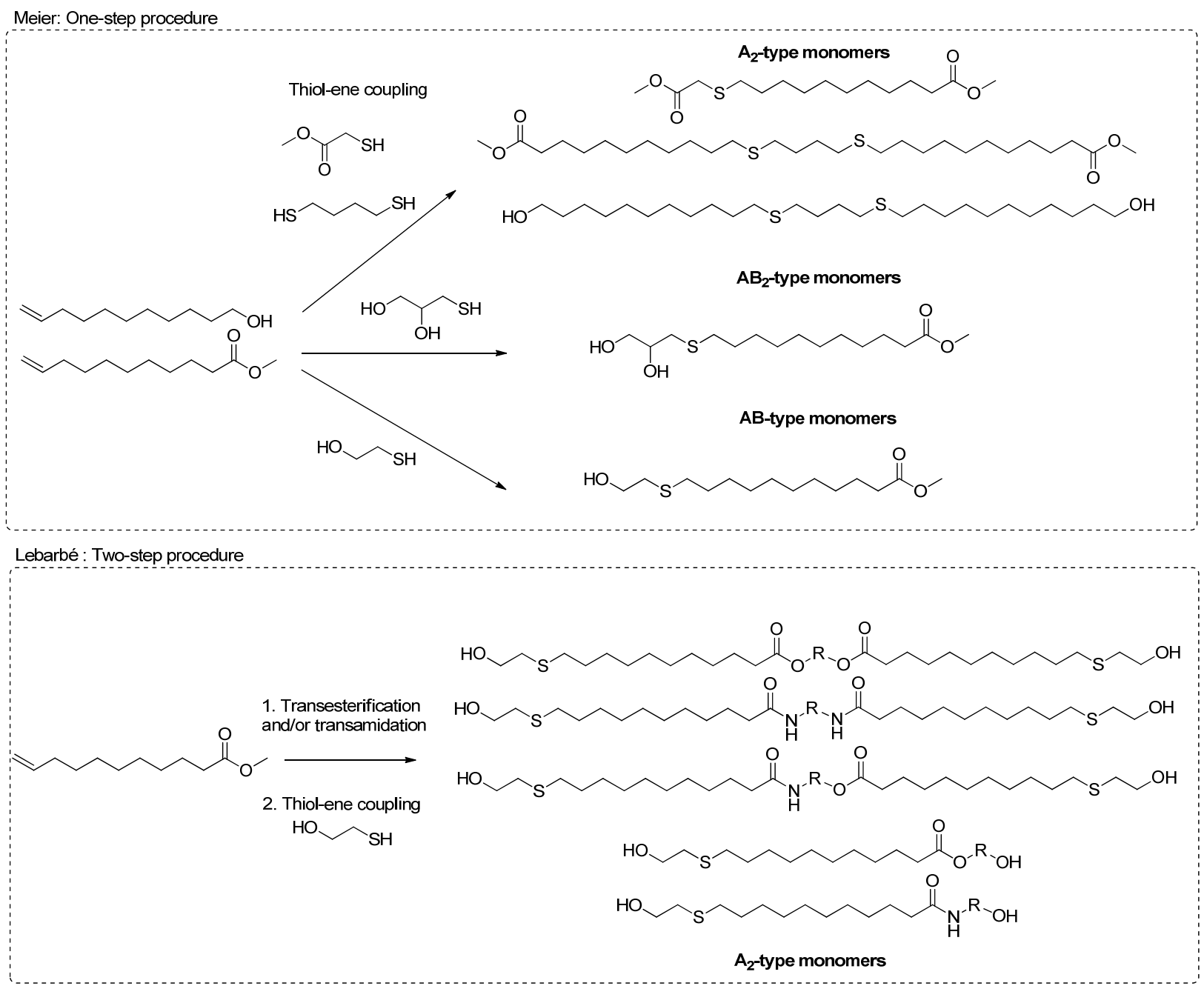

Figure 95. Monomer synthesis via thiol-ene click recactions.

Metathesis reactions were also used as a tool for the synthesis of dimers for polyester preparation. The self-metathesis of unsaturated fatty acid derivatives followed by other chemical modifications (hydrogenation, reduction of acid/ester functions into hydroxyl moieties) was conducted. ${ }^{521,522}$ For instance, fatty diacids were synthesized by self-metathesis of respectively 10 -undecenoic acid and erucic acid, followed by hydrogenation. The resulting dicarboxylic acids were polymerized with their corresponding diols (obtained after reduction) to yield PE-20,20 and PE-26,26. Besides, linear long aliphatic chain polyesters have been largely provided by ADMET polymerization ${ }^{251,521,523-525}$ of estercontaining $\alpha, \omega$-dienes, themselves prepared from fatty acids (Figure 96). Most of these studies conducted the hydrogenation of the resulting unsaturated polyesters with the aim of mimicking polyethylene 
structure. ${ }^{526}$ Moreover, Mecking and coll. ${ }^{521}$ demonstrated the strong impact of unsaturations within the polyesters that tends to decrease crystallinity and consequent melting temperature, justifying such hydrogenation process after polymerization.

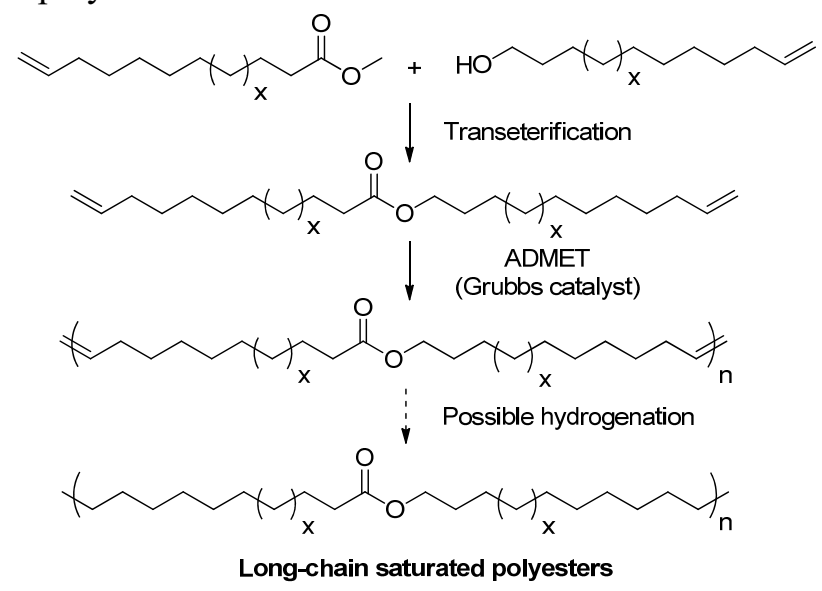

Figure 96. ADMET polymerization of fatty acid-based $\alpha, \omega$-dienes and possible hydrogenation towards saturated polyesters.

The group of Meier ${ }^{527}$ published a comparative study of the polymerization of fatty acid-based dienes containing ester functions via thiol-ene and ADMET processes. The authors demonstrated that thiol-ene click reaction was leading to higher molecular weight polyesters $\left(11850\right.$ g.mol $\left.{ }^{-1}\right)$ in comparison with ADMET polymerization (9000 g. $\left.\mathrm{mol}^{-1}\right)$ and proved the potential degradability of the synthesized polyesters in enzymatic and acidic conditions. Noteworthy, possible isomerization of the unsaturations during ADMET polymerization could create heterogeneities in the polyester structure decreasing its thermo-mechanical properties. Hopefully, the use of the first generation Grubbs catalyst and 1,4benzoquinone would limit this side reaction. ${ }^{528,529}$ Regarding thiol-ene coupling polymerizations, one of the major drawbacks is the presence of sulfur atoms in the resulting polymer backbone, leading to more flexible materials and to lower melting points.

The last common route to obtain polyesters is the ring opening polymerization of lactones that circumvent the stoichiometry issue encountered with AB-type polycondensation. Fatty acid-based macrolactones are less reactive than smaller size lactones and result in low molecular weight polyesters with high dispersities (around 1.4-1.5) after ROP. Additionally, these monomers are less readily available in comparison with the carboxylic acids from the same feedstock. Still, $\omega$-pentadecalactone and ethylene brassylate are both macrolactones naturally derived from Angelica archangelica L. root oil ${ }^{530}$ and musc ${ }^{531}$ respectively (Figure 97). As very few macrolactones are naturally occurring, other studies aimed at synthesizing fatty acid-based macrolactones using enzymatic or organic catalysts. ${ }^{532,533}$ For instance, ricinoleic acid was self-transesterified using dicyclohexylcarbodimide and (dimethylamino)pyridine as catalysts in order to produce the corresponding lactone (ricinoleic lactone, Figure 97) ${ }^{532}$ Regarding $\omega$ pentadecalactone, numerous studies ${ }^{534-539}$ reported the investigations on ROP catalysis of such bio-based compounds. The best results were achieved with Lipase Novozym 345 that displayed an extensive catalytic activity to ring-open this macrolactone, resulting in polyesters with molecular weight up to 470000 g.mol ${ }^{-1}$ 534-536 and high elongation at break around 100-200\%. Moreover, the biocompatibility and the non-toxicity of poly(pentadecalactone) was confirmed by Heise and coll. ${ }^{540}$ The group of Mecerreyes ${ }^{531}$ carried out an organocatalyst screening and demonstrated that TBD was able to efficiently catalyze the ROP of ethylene brassylate. Semi-crystalline aliphatic polyesters with molecular weight up to 13000 g. $\mathrm{mol}^{-1}$ and with slightly higher $\mathrm{Tm}$ and $\mathrm{Tg}$ than poly( $\varepsilon$-caprolactone) ones were achieved. 


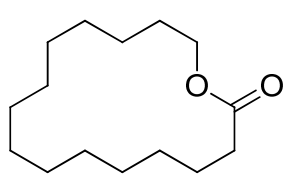

pentadecalactone

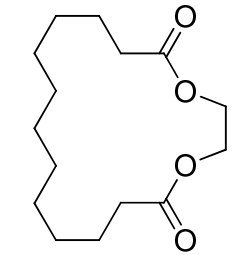

ethylene brassylate

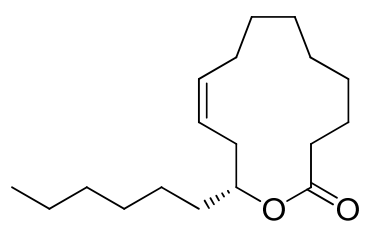

ricinoleic lactone

Figure 97. Examples of vegetable oil-based macro(di)lactones.

\section{Polycarbonates}

Aliphatic polycarbonates are very useful in a wide range of applications thanks to their biocompatibility, biodegradability and low toxicity. Only few examples of fatty acid-based polycarbonates have been reported.

The groups of Meier ${ }^{541}$ and Cramail ${ }^{542}$ developed a fatty acid-based carbonate-containing monomer from the transesterification between dimethyl carbonate and 10-undecenol. The resulting aliphatic diene was polymerized via ADMET polymerization and displayed molecular weights ranging in between 9500 and 50800 g.mol ${ }^{-1}$, depending on the reaction conditions chosen by both research teams.

Another strategy consisting in the copolymerization of dimethyl carbonate and bio-based diol was investigated. Miller and Vanderhenst ${ }^{543}$ carried out the reaction between dimethyl carbonate (or ethyl chloroformate) and 1,10-decanediol (sebacic acid derivative), in order to prepare an $\alpha, \omega$-bis-carbonate. Its catalyzed self-polycondensation led to polycarbonates with molecular weights in the range 8000-50000 g.mol ${ }^{-1}$. In the same study, the direct copolymerization of diol with dimethyl carbonate was realized and yielded polycarbonates with similar molar masses.

An original multi-step procedure involving (i) transcarbonation, (ii) epoxidation of the unsaturation and (iii) epoxide ring-opening (Figure 98) was developed by Cramail's group ${ }^{542}$ to design AB-type monomers from ricinoleic and oleic acids. The latter were subsequently polymerized under titanium tetrabutoxide catalysis leading to polycarbonates with molecular weights up to 14000 g.mol ${ }^{-1}$ and low Tg down to $-60^{\circ} \mathrm{C}$ due to the aliphatic chain plasticizer effect. 


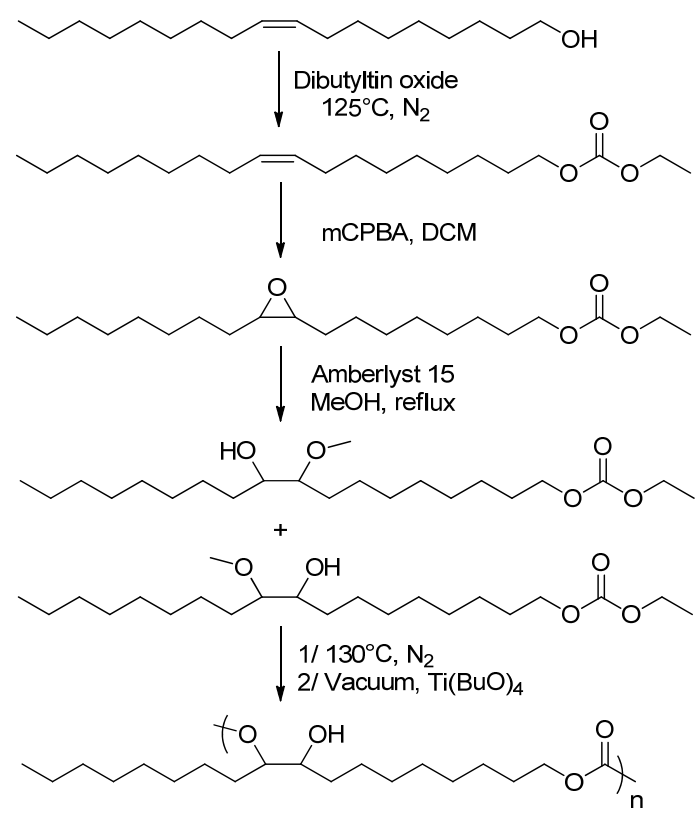

Figure 98. Multi-step synthesis of AB-type self-condensable monomer towards oleyl alcohol-based polycarbonate.

More recently, Zhang et al. ${ }^{544}$ carried out the selective copolymerization of $\mathrm{CO}_{2}$ with epoxy methyl 10undecenoate in the presence of a zinc-cobalt complex (Figure 99). Fatty acid-based polycarbonates were thus formed after epoxide ring-opening and displayed relatively low $\mathrm{Tg}$ due to internal plasticization imparted by aliphatic dangling chains and low molecular weight $\left(2000\right.$ g.mol $\left.{ }^{-1}\right)$. Consequently, the hydroxyl-terminated aliphatic polycarbonates were used to initiate ROP of L-lactide leading to an interesting biodegradable triblock PLLA-PC-PLLA.

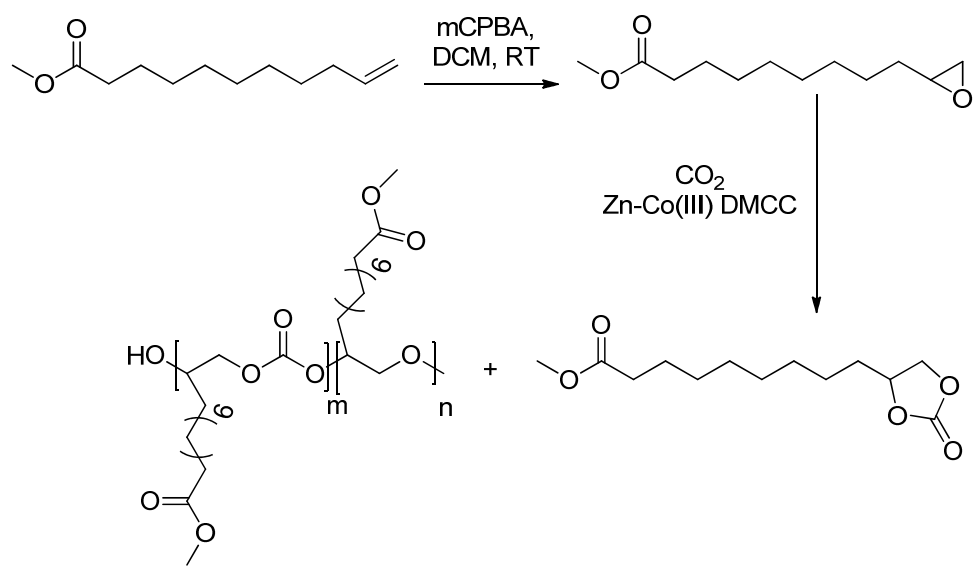

Figure 99. Copolymerization of epoxy methyl 10-undecenoate with $\mathrm{CO}_{2}$

\section{Polymers from terpenic resources}

\section{A. Introduction}


Terpenes, terpenoids and rosin are compounds that are mainly synthesized from plants. They all have in common one or more isoprene unit (2-methyl-1,4-butadiene) in their chemical structure. ${ }^{55}$ Although terpenes are mainly hydrocarbon components, terpenoids have additional constituents, for example, oxygen, containing functional groups such as carbonyl. Terpenes have been industrially important for years, particularly in the fine chemical and fragrance industries ${ }^{546}$ because they are abundant and inexpensive. Some of them even exhibit important pharmacological functions for the treaTment of many diseases, including cancer. ${ }^{547,548}$ In addition to these applications, terpenes are also widely used in polymer chemistry as starting materials. Here, some innovative polymeric materials based on terpene derivatives will be presented.

\section{B. Terpenes}

The most common terpenes can be isolated directly from pine trees and conifers. Indeed, the volatile fraction of resin, turpentine, is composed of a mixture of terpenes yielding directly $\alpha$ - and $\beta$-pinene and rosin. Turpentine is by far the main source of terpenes with a yearly terpenes production of 350000 tons $^{549}$. Other important terpenes as limonene or carvone can be produced by isomerization of $\alpha$ - or $\beta$ pinene $^{550,551}$ (Figure 100).

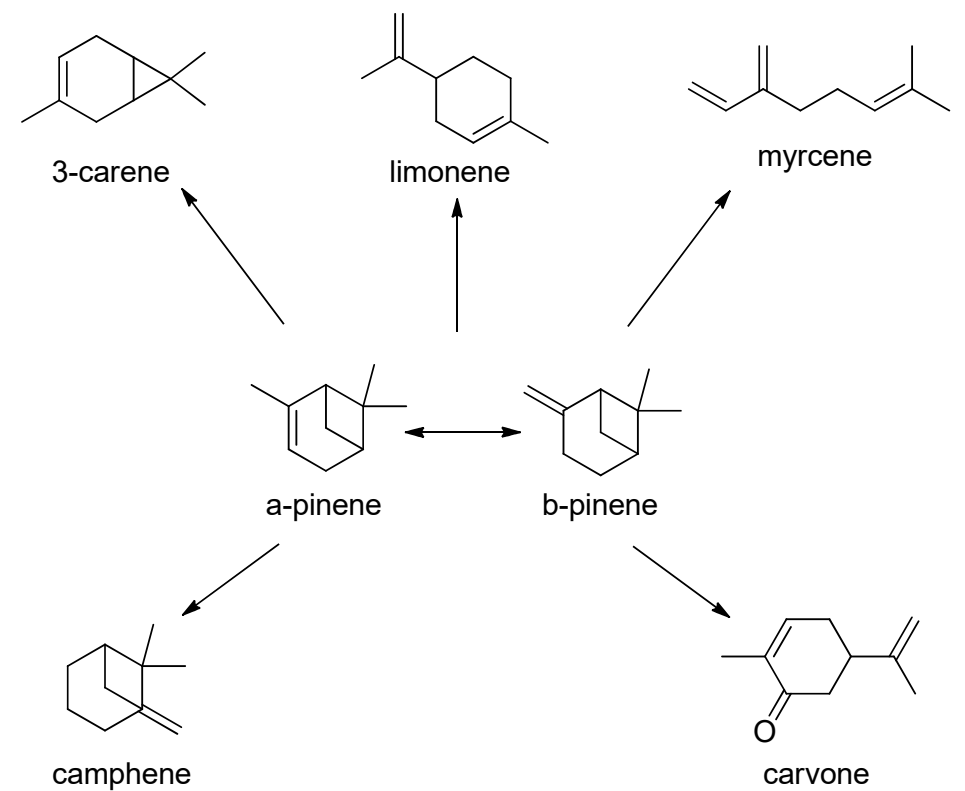

Figure 100. Various Terpenes derived from pinenes by isomerization

Thereby, only new polymers derived from $\alpha$-pinene, $\beta$-pinene, limonene and 2 linear terpenes (myrcene and citronellol) will be considered due to the low cost and the easy isolation of such starting materials.

\section{1. a-pinene-based polymers}

Pinenes are the most abundant and easily isolated terpenes. Obtained by the steam-distillation of sap from pine or conifer trees, pinenes can undergo a multitude of chemical reactions to produce valuable monomeric compounds. Structurally, $\alpha$-pinene is a bicyclic monoterpene hydrocarbon consisting of fourand six-membered rings with an internal trisubstituted carbon-carbon double bond in the six-membered ring (Figure 100). However, it is noteworthy that the majority of pinene polymerizations involve the use of $\beta$-pinene and not $\alpha$-pinene due to the lack of highly reactive terminal double bond in the $\alpha$-pinene structure. Consequently, only oligomers of $\alpha$-pinene can be obtained by direct cationic polymerization. 
The ring-opening metathesis polymerization (ROMP) could be a mean to access renewable materials from $\alpha$-pinene. However, its highly congested trisubstituted olefin avoids initiation and propagation steps. ${ }^{552}$ Nevertheless, Thomson and coll. ${ }^{553}$ investigated a novel method affording the ROMP of apopinene, an $\alpha$-pinene derivative. The authors have shown, for the first time, that apopinene can act as a monomer for pinene-derived ROMP processes to yield polymers with molecular weight up to 15600 $\mathrm{g} \cdot \mathrm{mol}^{-1}$.

The group of Kamigaito ${ }^{554}$ has also presented an innovative way to valorize $\alpha$-pinene into pinocarvone by chemical photo-oxidation under visible-light irradiation. Pinocarvone, which bears a reactive exo methylene group, can be quantitatively polymerized in a selective ring-opening radical process to afford novel bio-based polyketone (Figure 101).

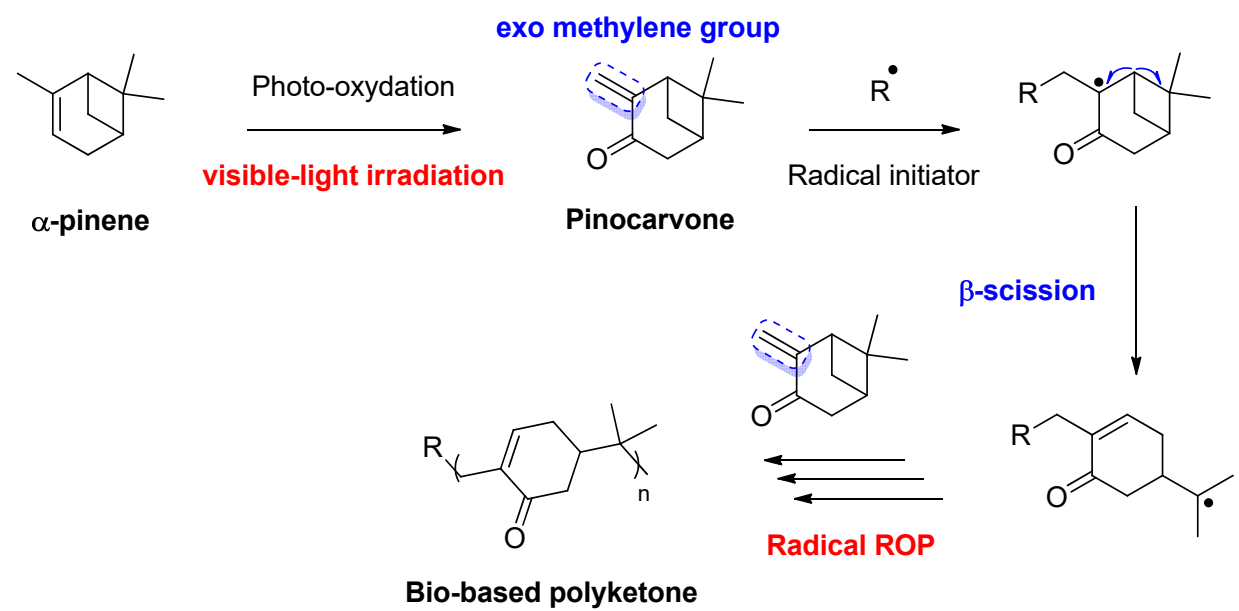

Figure 101. Structures of $\alpha$-pinene and pinocarvone and polymer thereof

An alternative way to create new renewable polymers from $\alpha$-pinene is described by Stockman and Howdle. ${ }^{555}$ Among other terpenes, the authors transformed $\alpha$-pinene into an acrylic and methacrylic monomer via a one-pot catalytic approach. The monomer was then polymerized into useful materials including polymer coatings.

\section{2. $\beta$-pinene-based polymers}

As previously mentioned, $\beta$-pinene is a readily suitable monomer for cationic polymerization. The mechanism is well understood, as well as the side reactions lowering the degree of polymerization. Indeed, the cationic polymerization of $\beta$-pinene employed a Lewis acid as a catalyst. These polymerizations resulted in only low molecular weight polymers $\left(\mathrm{M}_{n} \leq 4000\right.$ g.mol $\left.{ }^{-1}\right)$ which can be further modified to produce epoxy networks and polyols. ${ }^{556}$ However, numerous studies have been done to increase the molecular weight of poly( $\beta$-pinene). Among them, Kukhta et al ${ }^{557}$ produced relatively high molecular weight poly $\left(\beta\right.$-pinene) $\left(\mathrm{M}_{\mathrm{n}}=9000-14000 \mathrm{~g} \cdot \mathrm{mol}^{-1}\right)$, with a $\mathrm{Tg}$ in the range $82-91{ }^{\circ} \mathrm{C}$ using a complex co-initiator system comprising water, aluminum chloride and diphenyl ether at $20^{\circ} \mathrm{C}$ (Figure 102). 


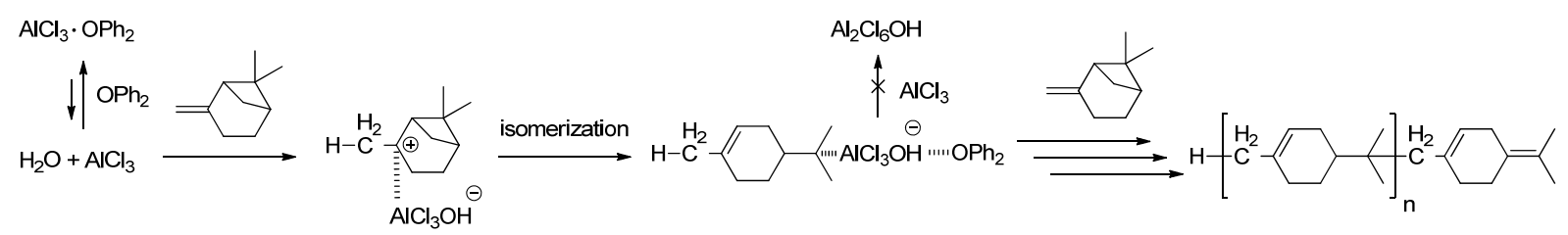

Figure 102. Proposed mechanism for $\beta$-pinene polymerization using $\mathrm{H}_{2} \mathrm{O} / \mathrm{AlCl}_{3} \cdot \mathrm{OPh}_{2}$ initiating system

One classical method to valorize $\beta$-pinene is its copolymerization with other monomers. Various structures were tested to afford new interesting copolymers presenting a large range of properties. The copolymerization of $\beta$-pinene with methyl acrylate (MA) was investigated by Wang et al. ${ }^{558}$ The feasibility of $\beta$-pinene and MA was demonstrated for the first time despite the significant difference between their reactivity ratios. The authors showed that the addition of a Lewis acid, $\mathrm{Et}_{2} \mathrm{AlCl}$, to the AIBN-initiated copolymerization enhanced the incorporation of $\beta$-pinene (up to $45 \mathrm{~mol} . \%$ ) but decreased the polymer molecular weight.

\section{Limonene-based polymers}

Another interesting terpene is limonene. As shown in Figure 100, limonene can be obtained from the isomerization of pinene, but this chiral molecule is also naturally produced by plants. The (R)- enantiomer consists of $90-96 \%$ of citrus peel oil and its worldwide production exceeds 70000 tons per year. Although the main use for limonene is in flavor and fragrance industry, its polymeric potential gains interest in academic research. For instance, Meier and coll. described the synthesis of new limonenebased polyesters ${ }^{559}$, polyamides ${ }^{560}$ and polyurethanes ${ }^{560}$ via thiol-ene additions. For the synthesis of polyesters, ester or alcohol functionalized thiols were added regioselectively to limonene to yield monofunctional, difunctional or heterodifunctional monomers, which are interesting renewable building blocks. On the other hand, cysteamine hydrochloride was used to produce amine-functionalized monomers for polyamide or polyurethane syntheses. Thus, limonene-based polymers were prepared with mixing above-mentioned monomers with ester or alcohol functionalized fatty acids.

Limonene-based polycarbonates can be also obtained from limonene oxide. The epoxide is commercially available as a mixture of the trans and cis diastereomers. Its abundance, low cost, and structural similarity to cyclohexene oxide make (R)-limonene oxide an excellent choice as a biorenewable epoxide monomer for copolymerization with $\mathrm{CO}_{2}$. The group of Coates ${ }^{561}$ described the alternating copolymerization of limonene oxide and $\mathrm{CO}_{2}$ catalyzed by a $\beta$-diiminate zinc molecule to give a new biodegradable polycarbonate, proceeding with highly selective incorporation of the trans diastereomer under mild conditions (Figure 103).

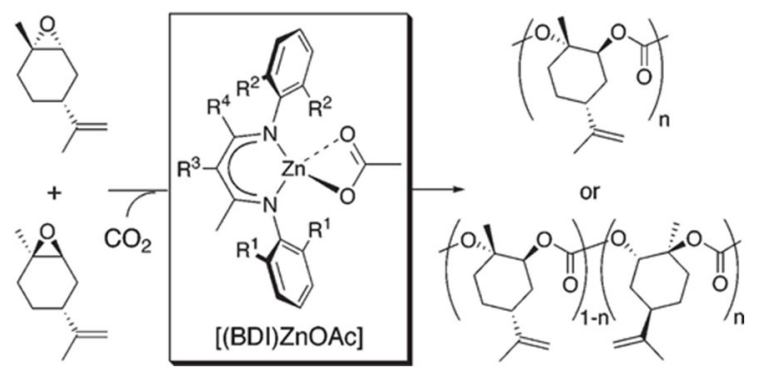

Figure 103. Copolymerization of limonene oxide and $\mathrm{CO}_{2}$ to produce polycarbonate 
Based on this study, Greiner' group ${ }^{562}$ managed to increase significantly the polycarbonate molecular weight from $25 \mathrm{~kg} \cdot \mathrm{mol}^{-1}$ to more than $100 \mathrm{~kg} \cdot \mathrm{mol}^{-1}$ with synthesizing the limonene oxide with very high content $(>85 \%)$ of trans-isomer and by masking hydroxyl functions in monomer impurities. Greiner also demonstrated that the bio-based poly(limonene carbonate) (PLimC) is a powerful platform to create numerous new functional materials. ${ }^{563}$ Different chemical transformations of the PLimC led to dramatic changes of its properties. For instance, a rubbery material was synthesized from the thermoplastic PLimC. Polymers with an antibacterial activity, increased hydrophilicity or even water solubility were also prepared from PLimC. The heat processability can be also improved after full hydrogenation of the polymer.

Finally, Mülhaupt ${ }^{564}$ reported a new route to linear and thermoset terpene-based non-isocyanate poly(hydroxyurethane)s, (NIPUs) derived from limonene dicarbonate.

\section{Linear terpenes-based polymers}

As shown in Figure 100, various monoterpenes are derived from the isomerization of pinene. One of these isomers, myrcene, has gained interest in recent years. Hillmyer et al. ${ }^{565}$ prepared high-molecular weight poly(3-methylenecyclopentene) using a combination of ring-closing metathesis and cationic polymerization with zinc chloride (Figure 104). This system afforded polymers with narrow molecular weight distributions. Furthermore, the molecular weight values could be tuned by changing the feed molar ratios of monomer to initiator, indicating that the polymerization proceeds in a controlled manner.

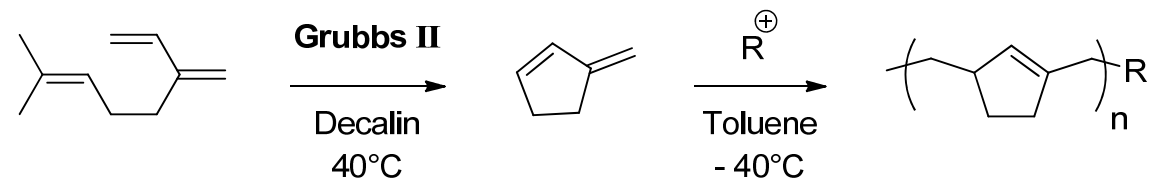

Figure 104. Ring-closing metathesis of myrcene followed by cationic polymerization of 3methylenecyclopentene $\mathrm{s}^{565}$

Another linear terpene, the citronellol, a major component of geranium and rose oils, can be directly oxidized into citronellic acid. This acid can be dimerized via an isomerizing alkoxycarbonylation reaction. ${ }^{566}$ The reactive primary carboxy group formed allows the preparation of novel high molecular weight polyesters.

\section{Terpenoids}

A second class of terpenes, referred to as terpenoids, possess functional groups that can be converted to a cyclic ester. Carvone and menthol are two common terpenoids (Figure 105) that have been successfully derivatized and further polymerized by ROP.<smiles>C=C(C)C1CC=C(C)C(=O)C1</smiles>

Carvone<smiles>CC1CCC(C(C)C)C(O)C1</smiles>

Menthol

Figure 105. Two common terpenoids: carvone and menthol 
Carvone is a natural product found in both Mentha spicata (spearmint) and Carum carvi (caraway) oils. The world production is estimated around 10000 tons annually. Carvone is mainly used as a flavor in food and beverages, as well as in toothpaste. After hydrogenation, carvone leads to dihydrocarvone and carvomenthone, two ketones that can be derivatized into lactones (dihydrocarvide and carvomenthide respectively) by Baeyer-Villiger oxidation. These lactones were polymerized using the catalyst/initiating system diethyl zinc/benzyl alcohol to yield aliphatic polyesters with low Tgs and a good control of the polymer molecular weights ${ }^{567}$ (Figure 106).

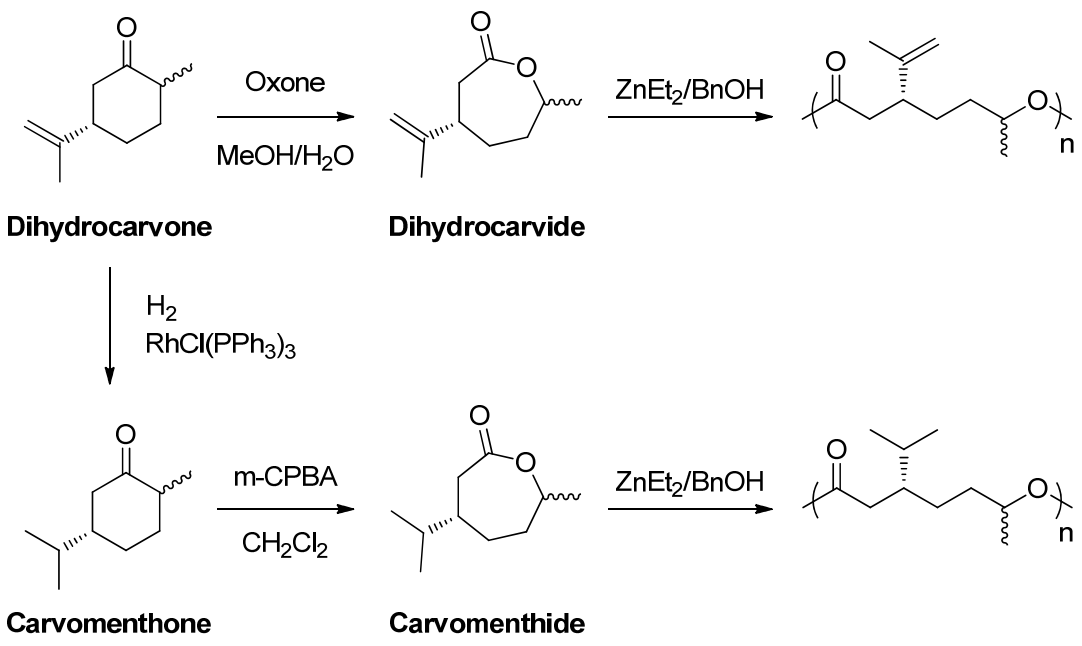

Figure 106. Baeyer-Villiger oxidation and ROP of carvone-derived monomers

The same group oxidized dihydrocarvone to an epoxylactone on a multigram scale. ${ }^{568}$ The resulting epoxylactone was used as a multifunctional monomer and as a cross-linker in ring-opening polymerizations leading to oligomers. However, copolymerizations of $\varepsilon$-caprolactone and the epoxylactone gave flexible cross-linked materials with shape memory properties. Associating the fact that these materials are biodegradable and biocompatible, they are valuable candidates for biomedical applications.

On the other hand, menthol is found in Mentha arvenis and Mentha piperita (peppermint) oils. Its primary uses are in the flavoring industry, as well as in various medicinal applications. Menthone is readily converted to a lactone monomer, menthide, by the Baeyer-Villiger oxidation. The resulting monomer can undergo ROP with a zinc alkoxide catalyst. ${ }^{569}$ The polymerization can take place at room temperature due to the high activity of the catalyst and the polymer molecular weight can be controlled by adjusting the monomer to catalyst ratio. The same group also worked on the synthesis of new ABA triblock copolymers prepared using the renewable monomers menthide and lactide by sequential ring-opening polymerizations. ${ }^{570,571}$ The authors demonstrated that the properties of the new biorenewable thermoplastic elastomers can be systematically modulated by changing the stereochemistry of the polylactide end blocks. The triblock copolymers can also act as pressure-sensitive adhesive when they are combined with renewable tackifier. ${ }^{572}$

\section{Rosin}

Rosin is a general term that designates three types of rosin types. Gum rosin is the most common type of rosin extracted from pine resin. The production of rosin is more than 1 million ton per year. Gum rosin is mainly composed of abietic-type and primaric-type acids as illustrated in Figure 107 while the 
predominant rosin acid is abietic acid. Rosin acids can be converted to a wide range of derivatives from esters and maleic anhydride adducts to hydrogenated acids. These low cost natural products have been used in anti-fouling, adhesive and ink applications.

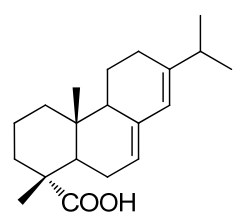

Abietic acid

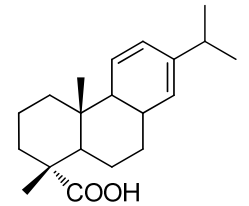

Levopimaric acid

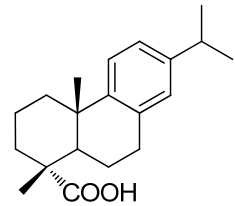

dehydroabietic acid

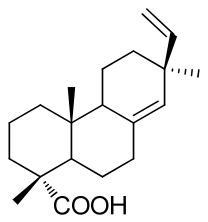

Pimaric acid

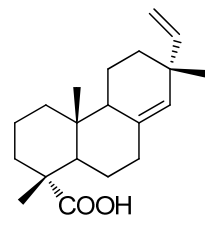

Isopimaric acid

Figure 107. Most common acids in gum rosin

Several groups have developed main chain rosin-based derived polymers obtained by step-growth polymerization. The nature of the monomer can be tuned, often involving a Diels-Alder reaction between levopimaric acid and a dienophile such as acrylic acid, maleic anhydride and maleimide. The resulting monomers can react with other comonomers such as diol, diacid, diamine, etc. affording the synthesis of polyesters, polyamides, polyamideimides and polyester polyols.

Atta et $a l .{ }^{573}$ described the formation of polyamide and polyamideimide using respectively a diacid functionalized levopimaric acid and an acid-anhydride functionalized levopimaric acid. The same group prepared rosin-based unsaturated polyesters ${ }^{574}$ using the same diacid functionalized levopimaric acid, ethylene glycol, maleic anhydride and adipic acid as the starting materials. The final unsaturated polymers were able to undergo curing in the presence of styrene to yield a cross-linked polymer with a $\mathrm{Tg}$ of $117^{\circ} \mathrm{C}$ potentially used in the field of steel coating.

Mustata group reported the synthesis of various polymers that can be obtained from this platform like water soluble poly(amide-imide) $\mathrm{s}^{575}$ and polyamides ${ }^{576,577}$, polyhydroxyimides ${ }^{578}$ and polyesters. ${ }^{579} \mathrm{Kim}$ et $a l .{ }^{580}$ have carried out studies on the formation of photoactive polyamide-imide. The polymer was made by the condensation of a maleopimaric adduct with azo-dye type diamines. Fabricated polymer films are very smooth, tough, adhesive to the substrate and optically clear.

As above mentioned, main-chain rosin-based polymers can be prepared by different step-growth polymerization methods. However, due to steric hindrance, monomer impurities and stoichiometric control, only low molecular weight polymers could be obtained. In order to access to higher molecular weights, an alternative strategy can be applied. Instead of targeting main-chain rosin-based polymers, different research group investigated the synthesis of side chain rosin-based polymers by radical polymerization. Thus, a platform of rosin-derived vinylic, allylic or acrylic monomers were developed, to be polymerized by radical polymerization.

Free radical polymerization was firstly used to obtain side chain rosin-based polymers. Only low molecular weight polymers were obtained with vinyl monomers because of the steric hindrance. To reduce the steric hindrance, allylic and acrylic monomers were synthesized. Only the latter showed 
promising results thanks to the higher reactivity of the double bond. ${ }^{581,582,583,584}$ In order to obtain welldefined polymers, controlled radical polymerization (CRP) was used thereafter. This route allows the control of polymers molecular weight and polymer architectures. RAFT (Reversible AdditionFragmentation chain Transfer) polymerization and ATRP (Atom-Transfer Radical-Polymerization) are two of the most widely used CRP methods. The group of Tang ${ }^{585-588}$ prepared side chain rosin-based polymers using both methods. For instance, the authors synthesized cationic rosin-containing methacrylate polymers with controlled molar mass, potentially used as antimicrobial agents ${ }^{588}$ (Figure 108).

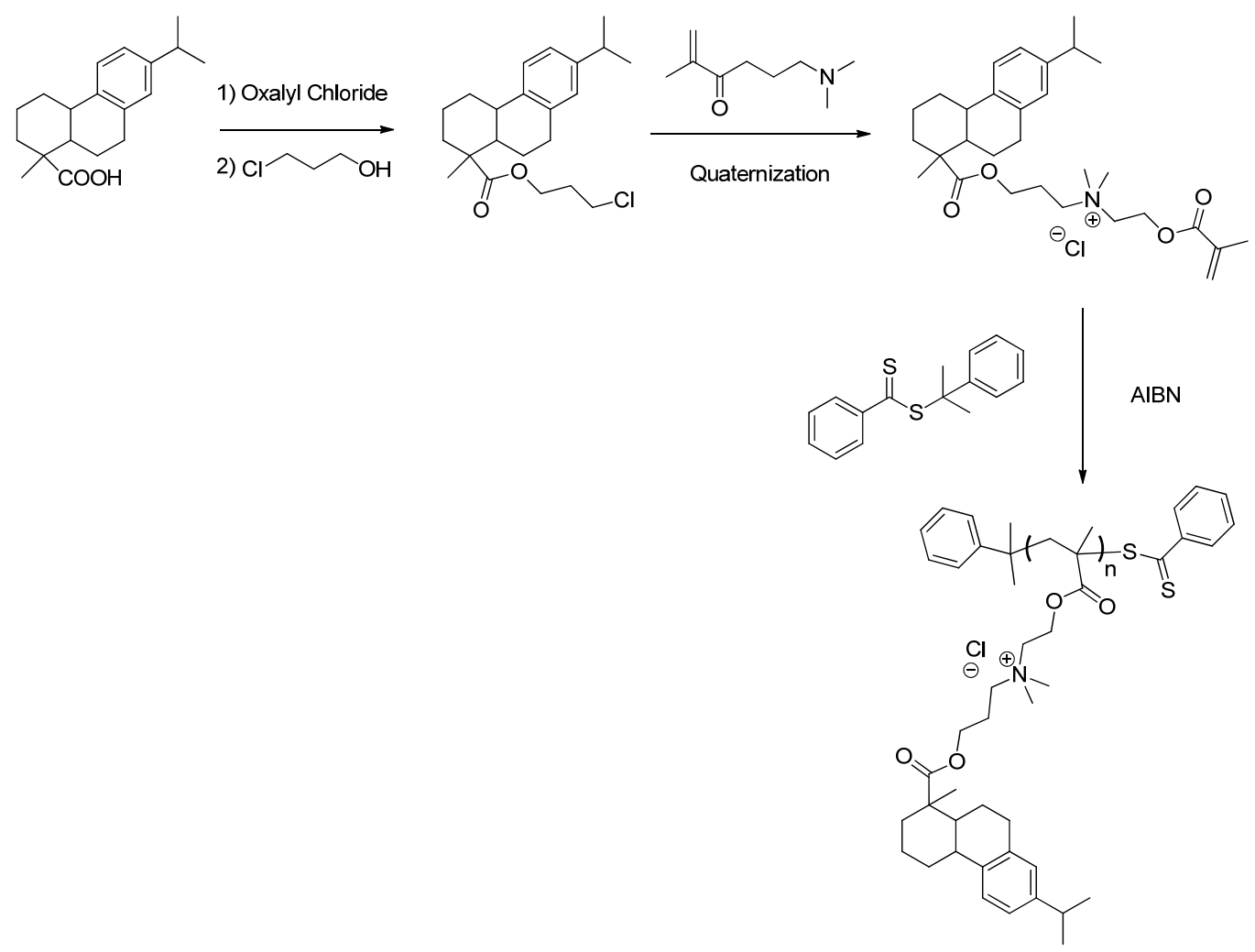

Figure 108. Cationic methacrylic polymers with natural rosin as pendant group

The major inconvenience of using controlled radio polymerization methods is the need to purify the raw rosin to obtain a well-defined resin acid. To avoid the purification step, Tang and co-workers ${ }^{589,590}$ combined ROP and click chemistry to prepare graft copolymers using raw rosin materials. The rosin properties have been successfully imparted to the initial polymers.

Last but not least, rosin has been widely used as curing agents to replace some of petroleum-derived ones. To do so, rosin has been derivatized to contain anhydrides, carboxyl or epoxy groups required for curing. The aromatic and cycloaliphatic structure of rosin acids affords the creation of rigid curing agents. Carlotti and co-workers ${ }^{591}$ worked on the synthesis rosin acid oligomers as precursor for epoxy resins. Zhang and co-workers ${ }^{592-595}$ also developed new rosin-derived rigid curing agents opening new opportunities in the field of thermosets.

\section{V.General conclusion}


At present the development of synthons (building-blocks) stemming from the biomass is rapidly expanding, both in the academic community and industry. The production of biosourced polymers is a real challenge in the coming years to reduce the environmental impacts. If numerous ways are ongoing, some products are already commercial: from vegetable oils, as PA-11 produced by Arkema or functional intermediaries by Elevance and from sugar derivatives such as lactic acid, produced by Natureworks, or isosorbide, produced by Roquette, to mention but a few.

However, beyond the scientific questions connected to the treaTment of the biomass, some challenges remain with respect to the development of the renewable resources for a sustainable chemistry.

First of all, it becomes now obvious that the mimetic way (molecules equivalent to those stemming from the petrochemistry) is not necessarily the most interesting. Indeed, most of the manufacturers choose original biosourced polymers while targeting new properties and functionalities. The only presence of renewable carbon is valuable only for very specific applications and does not justify systematically the biosourcing. Especially as the mimetic way leads to intermediaries that possess exactly the same issues as their fossil counterparts, without solving the questions of reduction of exposure levels in the dangerous compounds, for instance. On the other hand, the huge potential of the structures and the functionalities stemming from renewable resources allows to overtake certain compromises of properties, what is very sought by an innovative industry. Another major challenge consists in justifying the reduction of impact realized by the use of renewable resources. The life cycle analysis (LCA) takes here all its sense, in particular with regard to water footprint recently integrated (ISO 14046). The LCA enables to integrate, well upstream, the impacts connected to the production and to the collection of the renewable resources (inputs, transfers in fields, etc.). It emerges from these LCA that the use of renewable resources is not systematically a synonym of impact reduction. Indeed, the fossil polymers benefit from processes of polymerization (and monomer synthesis) optimized for more than 60 years and present the most reduced possible impacts. Actually, the synthesis of a kilogram of polypropylene, PP, presents at present less impact than the one of a kilogram of PLA. There are thus very wide margins of evolution for the improvement of the production processes of bio-sourced polymers. That is why the use of renewable resources has to proceed in particular of attentive choices concerning the level of crop productions, the choice and the volumes of biomass which can generate important environmental differences of impacts.

Therefore, the interest of bio-refineries takes all its sense to value at the most a given resource by integrating in particular the notion of valuation of the co-products and so to reduce the impacts and the costs. Some companies initially created at the heart of food-processing sectors as starch industry, certain stationeries, or distilleries are already organized in bio-refineries and value at the most their raw materials. The deployment of bio-refineries integrated in the territory, the judicious choice of their locations in agricultural basins and the optimization of their processes - by following the model of refineries - will guarantee the success of a large valuation of the renewable resources. This organization will permit to mutualize the costs, to make competitive the proposed intermediaries and to position with regard to hypothetical conflicts with the food use.

Also, the development of the valuation of the renewable resources - rich in atoms of oxygen in comparison to the fossil resources, generates a change of paradigm by positioning the catalytic reduction processes at the heart of the processing, which is based, in the case of fossil resources, essentially on the catalytic oxidation.

Finally, the choice of the resources and of the biosourced polymers must be also dictated by the variations of access to the oil resources. The development of the shale gas in the USA reduces the interest of the biosourced ethylene, but strengthens the needs for new bioresources, which can bring intermediaries with 
more than 4 carbon atoms, aromatic molecules and derivatives bearing long fatty chains. Furthermore, the use of renewable resources - globally rather available - allows to free from the business with oilproducing countries which exercise a cartelization of their production and thus permits to stabilize strategic supplies while favoring a long-lasting and sustainable agriculture, also by limiting the drift from the land and by regaining control grounds.

In conclusion, the development of polymers from renewable resources is inevitable and expects a colossal potential both for the academic research and the chemical industry in the coming years. All the world leader companies already launched into this competition by integrating chemistry and biotechnologies processes. This new research thematic is present from now in most of the laboratories and the conferences, justified by the Green Chemistry and by the current global environmental challenges. Besides, the challenges put by the production of monomers and biosourced polymers permit interdisciplinary crossings of interest with the experts of the biomass or the bioprocesses, which also take all their sense in a synergic approach of the biomass valuation. Finally, exceeding the hackneyed image of predation of the fossil resources, this chemistry based on the renewable resources can maybe give back to the chemistry all its sense of harmonious coexistence with the earth, foundation of its etymology: from the Egyptian kemet, the science and the knowledge of the earth for a sustainable future!

\section{Acknowledgments:}

HC most warmly thanks all the co-authors of this book chapter as well as Dr. Sylvain Caillol (Univ of Montpellier), Prof. Luc Avérous (Univ of Strasbourg), Prof. Stéphane Bruzaud (Univ of Bretagne Sud) and Prof. Stéphane Grelier (Univ. of Bordeaux) for helpful discussions. 


\section{References}

1. Fernando, S.; Adhikari, S.; Chandrapal, C.; Murali, N. Biorefineries: Current Status, Challenges, and Future Direction. Energy \& Fuels 2006, 20 (4), 1727-1737.

2. Gandini, A.; Belgacem, M. N. Furans in Polymer Chemistry. Prog. Polym. Sci. 1997, 22 (6), $1203-1379$.

3. Kamm, B.; Kamm, M.; Schmidt, M.; Hirth, T.; Schulze, M. Lignocellulose-Based Chemical Products and Product Family Trees; 2008; Vol. 2.

4. Rosatella, A. a.; Simeonov, S. P.; Frade, R. F. M.; Afonso, C. a. M. 5-Hydroxymethylfurfural (HMF) as a Building Block Platform: Biological Properties, Synthesis and Synthetic Applications. Green Chem. 2011, 13, 754.

5. Delidovich, I.; Hausoul, P. J. C.; Deng, L.; Pfützenreuter, R.; Rose, M.; Palkovits, R. Alternative Monomers Based on Lignocellulose and Their Use for Polymer Production. Chem. Rev. 2016, 116 (3), 1540-1599.

6. Brasholz, M.; von Känel, K.; Hornung, C. H.; Saubern, S.; Tsanaktsidis, J. Highly Efficient Dehydration of Carbohydrates to 5-(Chloromethyl)furfural (CMF), 5-(Hydroxymethyl)furfural (HMF) and Levulinic Acid by Biphasic Continuous Flow Processing. Green Chem. 2011, 13 (5), 1114.

7. Kohl, T. M.; Bizet, B.; Kevan, P.; Sellwood, C.; Tsanaktsidis, J.; Hornung, C. H. Efficient Synthesis of 5-(Chloromethyl)furfural (CMF) from High Fructose Corn Syrup (HFCS) Using Continuous Flow Processing. React. Chem. Eng. 2017, 2 (4), 541-549.

8. Dutta, S.; Wu, L.; Mascal, M. Production of 5-(Chloromethyl)furan-2-Carbonyl Chloride and Furan-2,5-Dicarbonyl Chloride from Biomass-Derived 5-(Chloromethyl)furfural (CMF). Green Chem. 2015, 17 (7), 3737-3739.

9. Mascal, M.; Nikitin, E. B. High-Yield Conversion of Plant Biomass into the Key Value-Added Feedstocks 5-(Hydroxymethyl)furfural, Levulinic Acid, and Levulinic Esters via 5(Chloromethyl)furfural. Green Chem. 2010, 12 (3), 370.

10. Bizet, B.; Hornung, C. H.; Kohl, T. M.; Tsanaktsidis, J. Synthesis of Imines and Amines from Furfurals Using Continuous Flow Processing. Aust. J. Chem. 2017, 70 (10).

11. Boisen, A.; Christensen, T. B.; Fu, W.; Gorbanev, Y. Y.; Hansen, T. S.; Jensen, J. S.; KliTgaard, S. K.; Pedersen, S.; Riisager, A.; Ståhlberg, T.; et al. Process Integration for the Conversion of Glucose to 2,5-Furandicarboxylic Acid. Chem. Eng. Res. Des. 2009, 87 (9), 1318-1327.

12. Haas, T.; Tacke, T.; Pfeffer, J. C.; Klasovsky, F.; Rimbach, M.; Volland, M.; Ortelt, M. Verfahren Zur Herstellung von 2,5-Diformylfuran Und Seiner Derivate. World Patent WO 2012004069 A1, $20120112,2012$.

13. Ma, J.; Xu, J.; Jia, X.; Wang, M.; Shi, S.; Miao, H.; Gao, J. The Method of Preparation of 2,5Dimethyl 2,5-Dimethyl Amino Acyl Furan Furans. Chinese Patent CN 104277018 A, 20150114, 2015.

14. Saha, B.; Bohn, C. M.; Abu-Omar, M. M. Zinc-Assisted Hydrodeoxygenation of Biomass-Derived 5-Hydroxymethylfurfural to 2,5-Dimethylfuran. ChemSusChem 2014, 7 (11), 3095-3101.

15. Cao, Q.; Liang, W.; Guan, J.; Wang, L.; Qu, Q.; Zhang, X.; Wang, X.; Mu, X. Catalytic Synthesis of 2,5-Bis-Methoxymethylfuran: A Promising Cetane Number Improver for Diesel. Appl. Catal. A Gen. 2014, 481, 49-53. 
16. Chatterjee, M.; Ishizaka, T.; Kawanami, H. Selective Hydrogenation of 5-Hydroxymethylfurfural to 2,5-Bis-(Hydroxymethyl)furan Using Pt/MCM-41 in an Aqueous Medium: A Simple Approach. Green Chem. 2014, 16 (11), 4734-4739.

17. Chen, J.; Lu, F.; Zhang, J.; Yu, W.; Wang, F.; Gao, J.; Xu, J. Immobilized Ru Clusters in Nanosized Mesoporous Zirconium Silica for the Aqueous Hydrogenation of Furan Derivatives at Room Temperature. ChemCatChem 2013, 5 (10), 2822-2826.

18. liu, F.; Audemar, M.; De Oliveira Vigier, K.; Clacens, J.-M.; De Campo, F.; Jerome, F. Combination of $\mathrm{Pd} / \mathrm{C}$ and Amberlyst-15 in a Single Reactor for the Acid/hydrogenating Catalytic Conversion of Carbohydrates to 5-Hydroxy-2,5-Hexanedione. Green Chem. 2014, 16 (9), 41104114.

19. Ohyama, J.; Esaki, A.; Yamamoto, Y.; Arai, S.; Satsuma, A. Selective Hydrogenation of 2Hydroxymethyl-5-Furfural to 2,5-Bis(hydroxymethyl)furan over Gold Sub-Nano Clusters. RSC Adv. 2013, 3 (4), 1033-1036.

20. Tamura, M.; Tokonami, K.; Nakagawa, Y.; Tomishige, K. Rapid Synthesis of Unsaturated Alcohols under Mild Conditions by Highly Selective Hydrogenation. Chem. Commun. 2013, 49 (63), 7034-7036.

21. Subbiah, S.; Simeonov, S. P.; Esperanca, J. M. S. S.; Rebelo, L. P. N.; Afonso, C. A. M. Direct Transformation of 5-Hydroxymethylfurfural to the Building Blocks 2,5-Dihydroxymethylfurfural (DHMF) and 5-Hydroxymethyl Furanoic Acid (HMFA) via Cannizzaro Reaction. Green Chem. 2013, 15 (10), 2849-2853.

22. Drewitt, J. G. N.; Lincoln, J. Polyesters from Heterocyclic Components. US Patent. US 2551731 19510508, 1951.

23. Papageorgiou, G. Z.; Papageorgiou, D. G.; Tsanaktsis, V.; Bikiaris, D. N. Synthesis of the BioBased Polyester Poly(propylene 2,5-Furan Dicarboxylate). Comparison of Thermal Behavior and Solid State Structure with Its Terephthalate and Naphthalate Homologues. Polymer (Guildf). 2015, $62,28-38$.

24. Papageorgiou, G. Z.; Tsanaktsis, V.; Bikiaris, D. N. Synthesis of Poly(ethylene Furandicarboxylate) Polyester Using Monomers Derived from Renewable Resources: Thermal Behavior Comparison with PET and PEN. Phys. Chem. Chem. Phys. 2014, 16 (17), 7946-7958.

25. Papageorgiou, G. Z.; Tsanaktsis, V.; Papageorgiou, D. G.; Exarhopoulos, S.; Papageorgiou, M.; Bikiaris, D. N. Evaluation of Polyesters from Renewable Resources as Alternatives to the Current Fossil-Based Polymers. Phase Transitions of Poly(butylene 2,5-Furan-Dicarboxylate). Polymer (Guildf). 2014, 55 (16), 3846-3858.

26. Zhu, J.; Cai, J.; Xie, W.; Chen, P.-H.; Gazzano, M.; Scandola, M.; Gross, R. A. Poly(butylene 2,5Furan Dicarboxylate), a Biobased Alternative to PBT: Synthesis, Physical Properties, and Crystal Structure. Macromolecules 2013, 46 (3), 796-804.

27. Ma, J.; Yu, X.; Xu, J.; Pang, Y. Synthesis and Crystallinity of Poly(butylene 2,5Furandicarboxylate). Polymer (Guildf). 2012, 53 (19), 4145-4151.

28. Papageorgiou, G. Z.; Tsanaktsis, V.; Papageorgiou, D. G.; Chrissafis, K.; Exarhopoulos, S.; Bikiaris, D. N. Furan-Based Polyesters from Renewable Resources: Crystallization and Thermal Degradation Behavior of Poly(hexamethylene 2,5-Furan-Dicarboxylate). Eur. Polym. J. 2015, 67, 383-396.

29. Jiang, M.; Liu, Q.; Zhang, Q.; Ye, C.; Zhou, G. A Series of Furan-Aromatic Polyesters Synthesized via Direct Esterification Method Based on Renewable Resources. J. Polym. Sci. Part A Polym. Chem. 2012, 50 (5), 1026-1036. 
30. Gubbels, E.; Jasinska-Walc, L.; Koning, C. E. Synthesis and Characterization of Novel Renewable Polyesters Based on 2,5-Furandicarboxylic Acid and 2,3-Butanediol. J. Polym. Sci. Part A Polym. Chem. 2013, 51 (4), 890-898.

31. Carman, H. S., J.; Killman, J. I., J.; Crawford, E. D.; Jenkins, J. C. Polyester Compositions Containing Furandicarboxylic Acid or an Ester Thereof and 2,2,4,4-Tetramethyl-1,3Cyclobutanediol. World Patent WO 2013055862 A1, 20130418, 2013.

32. Carman, H. S., J.; Killman, J. I., J.; Crawford, E. D.; Jenkins, J. C. Polyester Compositions Containing Furandicarboxylic Acid or an Ester Thereof, Cyclobutanediol and Ethylene Glycol. U.S. Patent US 20130095272 A1, $20130418,2013$.

33. Dong, W.; Chen, M.; Lan, D.; Yin, H.; Ni, Z.; Li, X. Preparation Method of Low-Yellowing 2,5Furandicarboxylic Acid-Based Polyester. Patent CN 102516513 B, 20120627, 2012.

34. Carlos Morales-Huerta, J.; Martínez de Ilarduya, A.; Muñoz-Guerra, S. Poly(alkylene 2,5Furandicarboxylate)s (PEF and PBF) by Ring Opening Polymerization. Polymer (Guildf). 2016, $87,148-158$.

35. de Jong, E.; Dam, M. A.; Sipos, L.; Gruter, G.-J. M. Furandicarboxylic Acid (FDCA), A Versatile Building Block for a Very Interesting Class of Polyesters. In Biobased Monomers, Polymers, and Materials; ACS Symposium Series; American Chemical Society, 2012; Vol. 1105, pp 1-13.

36. Burgess, S. K.; Wenz, G. B.; Kriegel, R. M.; Koros, W. J. Penetrant Transport in Semicrystalline Poly(ethylene Furanoate). Polymer (Guildf). 2016, 98, 305-310.

37. Burgess, S. K.; Kriegel, R. M.; Koros, W. J. Carbon Dioxide Sorption and Transport in Amorphous Poly(ethylene Furanoate). Macromolecules 2015, 48 (7), 2184-2193.

38. Burgess, S. K.; Mubarak, C. R.; Kriegel, R. M.; Koros, W. J. Physical Aging in Amorphous Poly(ethylene Furanoate): Enthalpic Recovery, Density, and Oxygen Transport Considerations. $J$. Polym. Sci. Part B Polym. Phys. 2015, 53 (6), 389-399.

39. Collias, D. I.; Harris, A. M.; Nagpal, V.; Cottrell, I. W.; Schultheis, M. W. Biobased Terephthalic Acid Technologies: A Literature Review. Ind. Biotechnol. 2014.

40. Lin, Z.; Ierapetritou, M.; Nikolakis, V. Aromatics from Lignocellulosic Biomass: Economic Analysis of the Production of P-Xylene from 5-Hydroxymethylfurfural. AIChE J. 2013, 59 (6), 2079-2087.

41. Zeng, C.; Seino, H.; Ren, J.; Yoshie, N. Polymers with Multishape Memory Controlled by Local Glass Transition Temperature. ACS Appl. Mater. Interfaces 2014, 6 (4), 2753-2758.

42. Zeng, C.; Seino, H.; Ren, J.; Hatanaka, K.; Yoshie, N. Self-Healing Bio-Based Furan Polymers Cross-Linked with Various Bis-Maleimides. Polymer (Guildf). 2013, 54 (20), 5351-5357.

43. Zeng, C.; Seino, H.; Ren, J.; Hatanaka, K.; Yoshie, N. Bio-Based Furan Polymers with SelfHealing Ability. Macromolecules 2013, 46 (5), 1794-1802.

44. Ikezaki, T.; Matsuoka, R.; Hatanaka, K.; Yoshie, N. Biobased poly(2,5-Furandimethylene Succinate-Co-Butylene Succinate) Crosslinked by Reversible Diels-Alder Reaction. J. Polym. Sci. Part A Polym. Chem. 2014, 52 (2), 216-222.

45. Jiang, Y.; WoorTman, A. J. J.; Alberda van Ekenstein, G. O. R.; Petrović, D. M.; Loos, K. Enzymatic Synthesis of Biobased Polyesters Using 2,5-Bis(hydroxymethyl)furan as the Building Block. Biomacromolecules 2014, 15 (7), 2482-2493.

46. Taarning, E.; Nielsen, I. S.; Egeblad, K.; Madsen, R.; Christensen, C. H. Chemicals from 
Renewables: Aerobic Oxidation of Furfural and Hydroxymethylfurfural over Gold Catalysts. ChemSusChem 2008, 1 (1-2), 75-78.

47. Casanova, O.; Iborra, S.; Corma, A. Biomass into Chemicals: One Pot-Base Free Oxidative Esterification of 5-Hydroxymethyl-2-Furfural into 2,5-Dimethylfuroate with Gold on Nanoparticulated Ceria. J. Catal. 2009, 265 (1), 109-116.

48. Pinna, F.; Olivo, A.; Trevisan, V.; Menegazzo, F.; Signoretto, M.; Manzoli, M.; Boccuzzi, F. The Effects of Gold Nanosize for the Exploitation of Furfural by Selective Oxidation. Catal. Today 2013, 203, 196-201.

49. Menegazzo, F.; Fantinel, T.; Signoretto, M.; Pinna, F.; Manzoli, M. On the Process for Furfural and HMF Oxidative Esterification over Au/ZrO2. J. Catal. 2014, 319, 61-70.

50. Menegazzo, F.; Signoretto, M.; Pinna, F.; Manzoli, M.; Aina, V.; Cerrato, G.; Boccuzzi, F. Oxidative Esterification of Renewable Furfural on Gold-Based Catalysts: Which Is the Best Support? J. Catal. 2014, 309, 241-247.

51. Signoretto, M.; Menegazzo, F.; Contessotto, L.; Pinna, F.; Manzoli, M.; Boccuzzi, F. Au/ZrO2: An Efficient and Reusable Catalyst for the Oxidative Esterification of Renewable Furfural. Appl. Catal. B Environ. 2013, 129, 287-293.

52. Pennanen, S.; Nyman, G. Studies on the Furan Series. Acta Chem. Scand. 1972, 26, 1018-1022.

53. Khrouf, A.; Boufi, S.; Gharbi, R. El; Belgacem, N. M.; Gandini, A. Polyesters Bearing Furan Moieties. Polym. Bull. 1996, 37 (5), 589-596.

54. Khrouf, A.; Abid, M.; Boufi, S.; El Gharbi, R.; Gandini, A. Polyesters Bearing Furan Moieties, 2: A Detailed Investigation of the Polytransesterification of Difuranic Diesters with Different Diols. Macromol. Chem. Phys. 1998.

55. Gharbi, S.; Andreolety, J.-P.; Gandini, A. Polyesters Bearing Furan Moieties: IV. Solution and Interfacial Polycondensation of 2,2'-bis(5-Chloroformyl-2-Furyl)propane with Various Diols and Bisphenols. Eur. Polym. J. 2000, 36 (3), 463-472.

56. Kamoun, W.; Salhi, S.; Rousseau, B.; El Gharbi, R.; Fradet, A. Furanic-Aromatic Copolyesters by Interchange Reactions between Poly(ethylene Terephthalate) and Poly[ethylene 5,5'Isopropylidene-bis(2-Furoate)]. Macromol. Chem. Phys. 2006, 207 (22), 2042-2049.

57. Pentz, W. J. Polyurethanes or Isocyanurates from Alkoxylated Hydroxymethylfuran. U.S. Patent US 4,426,460 A, 19840117, 1984.

58. Dunlop, W. R.; Pentz, W. J. Ow Fire Hazard Rigid Urethane Insulation Foam and Polyol Mixtures Used in Its Manufacture. Patent US 4,318,999 A, 19820309, 1982.

59. The Dow Chemical Company. Describe Dow's PAPI (TM) Polymeric MDI product offerings and the applications they may be used in.

60. Cawse, J. L.; Stanford, J. L.; Still, R. H. Polymers from Renewable Sources, 1. Diamines and Diisocyanates Containing Difurylalkane Moieties. Die Makromol. Chemie 1984, 185 (4), 697 707.

61. Cawse, J. L.; Stanford, J. L.; Still, R. H. Polymers from Renewable Sources, 2. Kinetics and Polyurethane Formation from Furan-Based Diisocynates. Die Makromol. Chemie 1984, 185 (4), 709-723.

62. Cawse, J. L.; Stanford, J. L.; Still, R. H. Novel Precursors Suitable for RIM Polyurethane Networks. Br. Polym. J. 1985, 17 (2), 233-238. 
63. Boufi, S.; Belgacem, M. N.; Quillerou, J.; Gandini, A. Urethanes and Polyurethanes Bearing Furan Moieties. 4. Synthesis, Kinetics and Characterization of Linear Polymers. Macromolecules 1993, 26 (25), 6706-6717.

64. Boufi, S.; Gandini, A.; Belgacem, M. N. Urethanes and Polyurethanes Bearing Furan Moieties: 5. Thermoplastic Elastomers Based on Sequenced Structures. Polymer (Guildf). 1995, 36 (8), 16891696.

65. Mitiakoudis, A.; Gandini, A. Synthesis and Characterization of Furanic Polyamides. Macromolecules 1991, 24 (4), 830-835.

66. Ortelt, M.; Spyrou, E.; Pfeffer, J. C.; Fuchsmann, D.; Kohlstruk, B.; Haas, T. Curable Compositions Based on Epoxy Resins without Benzyl Alcohol. World Patent WO 2014037222 A2, 20140313, 2014.

67. Amarasekara, A. S. 5-Hydroxymethylfurfural Based Polymers. In Renewable Polymers: Synthesis, Processing, and Technology; 2011.

68. Hui, Z.; Gandini, A. Polymeric Schiff Bases Bearing Furan Moieties. Eur. Polym. J. 1992, 28 (12), 1461-1469.

69. Méalares, C.; Gandini, A. Polymeric Schiff Bases Bearing Furan Moieties 2. Polyazines and Polyazomethines. Polym. Int. 1996, 40, 33-39.

70. Cooke, A. W.; Wagener, K. B. An Investigation of Polymerization via Reductive Coupling of Carbonyls. Macromolecules 1991, 24 (6), 1404-1407.

71. Choi, T.-L.; Han, K.-M.; Park, J.-I.; Kim, D. H.; Park, J.-M.; Lee, S. High Performance Organic Thin-Film Transistor Based on Amorphous A,B-Alternating Poly(arylenevinylene) Copolymers. Macromolecules 2010, 43 (14), 6045-6049.

72. Kim, C. Y.; Cho, H. N.; Kim, D. Y.; Kim, Y. C.; Lee, J. Y.; Kim, J. K. Fluorene-Based Alternating Copolymers and Electroluminescence Elements Incorporating Them. Patent GB 2313127 A, 19971119, 1997.

73. Ishida, K.; Furuhashi, Y.; Yoshie, N. Synthesis of Diels-Alder Network Polymers from Bisfuranic Terminated Poly(1-Lactide) and Tris-Maleimide. Polym. Degrad. Stab. 2014, 110, 149-155.

74. Vilela, C.; Cruciani, L.; Silvestre, A. J. D.; Gandini, A. Reversible Polymerization of Novel Monomers Bearing Furan and Plant Oil Moieties: A Double Click Exploitation of Renewable Resources. RSC Adv. 2012, 2 (7), 2966-2974.

75. Vilela, C.; Silvestre, A. J. D.; Gandini, A. Thermoreversible Nonlinear Diels-Alder Polymerization of Furan/plant Oil Monomers. J. Polym. Sci. Part A Polym. Chem. 2013, 51 (10), 2260-2270.

76. Banella, M.; Gioia, C.; Vannini, M.; Colonna, M.; Celli, A.; Gandini, A. A New Approach to the Synthesis of Monomers and Polymers Incorporating Furan/maleimide Diels-Alder Adducts; 2016; Vol. 1736.

77. Isikgor, F. H.; Becer, C. R. Lignocellulosic Biomass: A Sustainable Platform for the Production of Bio-Based Chemicals and Polymers. Polym. Chem. 2015.

78. Rose, M.; Palkovits, R. Cellulose-Based Sustainable Polymers: State of the Art and Future Trends. Macromol. Rapid Commun. 2011.

79. The Biofine Process - Production of Levulinic Acid, Furfural, and Formic Acid from Lignocellulosic Feedstocks. In Biorefineries-Industrial Processes and Products; Wiley-VCH 
Verlag GmbH, 2008; pp 139-164.

80. Mukherjee, A.; Dumont, M. J.; Raghavan, V. Review: Sustainable Production of Hydroxymethylfurfural and Levulinic Acid: Challenges and Opportunities. Biomass and Bioenergy. 2015.

81. The University of Maine - College of Engineering.

82. Mullen, B. D.; Scholten, M. D.; Mullen, T. J.; Leibig, C. M.; Badarinarayana, V. Polyketal Adducts, Methods of Manufacture and Uses Thereof. U.S. Patent 2012/0118201 A1, 2011.

83. Fotinos, N.; Campo, M. A.; Popowycz, F.; Gurny, R.; Lange, N. 5-Aminolevulinic Acid Derivatives in Photomedicine: Characteristics, Application and Perspectives. Photochem. Photobiol. 2006, 82 (4), 994-1015.

84. Bozell, J. J.; Moens, L.; Elliott, D. C.; Wang, Y.; Neuenscwander, G. G.; Fitzpatrick, S. W.; Bilski, R. J.; Jarnefeld, J. L. Production of Levulinic Acid and Use as a Platform Chemical for Derived Products. Resour. Conserv. Recycl. 2000, 28 (3), 227-239.

85. Serrano-Ruiz, J. C.; West, R. M.; Dumesic, J. A. Catalytic Conversion of Renewable Biomass Resources to Fuels and Chemicals. Annu. Rev. Chem. Biomol. Eng. 2010, 1 (1), 79-100.

86. Al-Shaal, M. G.; Wright, W. R. H.; Palkovits, R. Exploring the Ruthenium Catalysed Synthesis of [Gamma]-Valerolactone in Alcohols and Utilisation of Mild Solvent-Free Reaction Conditions. Green Chem. 2012, 14 (5), 1260-1263.

87. Wright, W. R. H.; Palkovits, R. Development of Heterogeneous Catalysts for the Conversion of Levulinic Acid to $\gamma$-Valerolactone. ChemSusChem 2012, 5 (9), 1657-1667.

88. Manzer, L. E. Catalytic Synthesis of $\alpha$-Methylene- $\gamma$-Valerolactone: A Biomass-Derived Acrylic Monomer. Appl. Catal. A Gen. 2004, 272 (1), 249-256.

89. Manzer, L.; Hutchenson, K. Process for the Production of Y-Methyl-a-Methylene-YButyrolactone from Reaction of Levulinic Acid and Hydrogen Followed by Reaction of Crude YValerolactone and Formaldehyde, Both Reactions Being Carried out in the Supercritical or nearCritical Fluid Ph. U.S. Patent US 200600100450 A1, 2006.

90. Marvel, C. S.; Levesque, C. L. The Structure of Vinyl Polymers. III.1 The Polymer from $\alpha$ Angelica Lactone. J. Am. Chem. Soc. 1939, 61 (7), 1682-1684.

91. Leonard, R. H. Method of Converting Levulinic Acid into Alpha Angelica Lactone. U.S. Patent US 2,809,203, 1957.

92. Ertl, J.; Cerri, E.; Rizzuto, M.; Caretti, D. Natural Derivatives of Diphenolic Acid as Substitutes for Bisphenol-A. AIP Conf. Proc. 2014, 1599 (1), 326-329.

93. Liu, H.-F.; Zeng, F.-X.; Deng, L.; Liao, B.; Pang, H.; Guo, Q.-X. Bronsted Acidic Ionic Liquids Catalyze the High-Yield Production of Diphenolic Acid/esters from Renewable Levulinic Acid. Green Chem. 2013, 15 (1), 81-84.

94. Arasa, M.; Pethrick, R. A.; Mantecón, A.; Serra, A. New Thermosetting Nanocomposites Prepared from Diglycidyl Ether of Bisphenol and $\gamma$-Valerolactone Initiated by Rare Earth Triflate Initiators. Eur. Polym. J. 2010, 46 (1), 5-13.

95. Lee, C. W.; Urakawa, R.; Kimura, Y. Copolymerization of $\gamma$-Valerolactone and $\beta$-Butyrolactone. Eur. Polym. J. 1998, 34 (1), 117-122.

96. Chalid, M.; Heeres, H. J.; Broekhuis, A. A. Green Polymer Precursors from Biomass-Based 
Levulinic Acid. Procedia Chem. 2012, 4, 260-267.

97. Alonso, D. M.; Bond, J. Q.; Dumesic, J. A. Catalytic Conversion of Biomass to Biofuels. Green Chem. 2010, 12 (9), 1493-1513.

98. Rothen-Weinhold, A.; Schwach-Abdellaoui, K.; Barr, J.; Ng, S. Y.; Shen, H.-R.; Gurny, R.; Heller, J. Release of BSA from Poly(ortho Ester) Extruded Thin Strands. J. Control. Release 2001, $71(1), 31-37$.

99. Lange, J.-P.; Vestering, J. Z.; Haan, R. J. Towards "Bio-Based" Nylon: Conversion of [Gamma]Valerolactone to Methyl Pentenoate under Catalytic Distillation Conditions. Chem. Commun. 2007, No. 33, 3488-3490.

100. Tarabanko, V. E.; Kaygorodov, N. K. L. Chemistry for Sustainable Development 18 (2010) 321328 New Biodedradable Polymers Based on $\alpha \alpha \alpha \alpha \alpha$-Angelicalactone.

101. Chen, T.; Qin, Z.; Qi, Y.; Deng, T.; Ge, X.; Wang, J.; Hou, X. Degradable Polymers from RingOpening Polymerization of [Small Alpha]-Angelica Lactone, a Five-Membered Unsaturated Lactone. Polym. Chem. 2011, 2 (5), 1190-1194.

102. Qi, G.; Nolan, M.; Schork, F. J.; Jones, C. W. Emulsion and Controlled Miniemulsion Polymerization of the Renewable Monomer $\gamma$-Methyl- $\alpha$-Methylene- $\gamma$-Butyrolactone. J. Polym. Sci. Part A Polym. Chem. 2008, 46 (17), 5929-5944.

103. Miyake, G. M.; Zhang, Y.; Chen, E. Y.-X. Living Polymerization of Naturally Renewable Butyrolactone-Based Vinylidene Monomers by Ambiphilic Silicon Propagators. Macromolecules 2010, 43 (11), 4902-4908.

104. Gowda, R. R.; Chen, E. Y.-X. Chiral and Achiral (Imino)phenoxy-Based Cationic Group 4 NonMetallocene Complexes as Catalysts for Polymerization of Renewable [Small Alpha]-Methylene[Gamma]-Butyrolactones. Dalt. Trans. 2013, 42 (25), 9263-9273.

105. Hu, Y.; Xu, X.; Zhang, Y.; Chen, Y.; Chen, E. Y.-X. Polymerization of Naturally Renewable Methylene Butyrolactones by Half-Sandwich Indenyl Rare Earth Metal Dialkyls with Exceptional Activity. Macromolecules 2010, 43 (22), 9328-9336.

106. Hu, Y.; Miyake, G. M.; Wang, B.; Cui, D.; Chen, E. Y.-X. Ansa-Rare-Earth-Metal Catalysts for Rapid and Stereoselective Polymerization of Renewable Methylene Methylbutyrolactones. Chem. - A Eur. J. 2012, 18 (11), 3345-3354.

107. Schmitt, M.; Falivene, L.; Caporaso, L.; Cavallo, L.; Chen, E. Y.-X. High-Speed Organocatalytic Polymerization of a Renewable Methylene Butyrolactone by a Phosphazene Superbase. Polym. Chem. 2014, 5 (9), 3261-3270.

108. Zhang, Y.; Miyake, G. M.; Chen, E. Y.-X. Alane-Based Classical and Frustrated Lewis Pairs in Polymer Synthesis: Rapid Polymerization of MMA and Naturally Renewable Methylene Butyrolactones into High-Molecular-Weight Polymers. Angew. Chemie Int. Ed. 2010, 49 (52), 10158-10162.

109. Xu, T.; Chen, E. Y.-X. Probing Site Cooperativity of Frustrated Phosphine/Borane Lewis Pairs by a Polymerization Study. J. Am. Chem. Soc. 2014, 136 (5), 1774-1777.

110. Chen, X.; Caporaso, L.; Cavallo, L.; Chen, E. Y.-X. Stereoselectivity in Metallocene-Catalyzed Coordination Polymerization of Renewable Methylene Butyrolactones: From Stereo-Random to Stereo-Perfect Polymers. J. Am. Chem. Soc. 2012, 134 (17), 7278-7281.

111. Hu, Y.; Gustafson, L. O.; Zhu, H.; Chen, E. Y.-X. Anionic Polymerization of MMA and Renewable Methylene Butyrolactones by Resorbable Potassium Salts. J. Polym. Sci. Part A 
Polym. Chem. 2011, 49 (9), 2008-2017.

112. Tagle, L. H.; Diaz, F. R.; Donoso, A. Polymerization by Phase-Transfer Catalysis. Part 20. Synthesis of Polycarbonates and Polythiocarbonates with an Ester Group in the Side Chain. $J$. Macromol. Sci. Part A 1996, 33 (11), 1643-1651.

113. Moore, J. A.; Tannahill, T. Homo- and Co-Polycarbonates and Blends Derived from Diphenolic Acid. High Perform. Polym. 2001.

114. Zhang, R.; Moore, J. A. Synthesis, Characterization and Properties of Polycarbonate Containing Carboxyl Side Groups. Macromol. Symp. 2003, 199 (1), 375-390.

115. Zhou, S.; Kim, D. Cross-Linked Aryl-Sulfonated Poly(arylene Ether Ketone) Proton Exchange Membranes for Fuel Cell. Electrochim. Acta 2012, 63, 238-244.

116. Maiorana, A.; Spinella, S.; Gross, R. A. Bio-Based Alternative to the Diglycidyl Ether of Bisphenol A with Controlled Materials Properties. Biomacromolecules 2015, 16 (3), 1021-1031.

117. Patel, A.; Maiorana, A.; Yue, L.; Gross, R. A.; Manas-Zloczower, I. Curing Kinetics of Biobased Epoxies for Tailored Applications. Macromolecules 2016, 49 (15), 5315-5324.

118. Chu, F.; Hawker, C. J.; Pomery, P. J.; Hill, D. J. T. Intramolecular Cyclization in Hyperbranched Polyesters. J. Polym. Sci., Part A Polym. Chem. 1997, 35, 1627-1633.

119. Kambouris, P.; Hawker, C. J. A Versatile New Method for Structure Determination in Hyperbranched Macromolecules. J. Chem. Soc. Perkin Trans. 1 1993, No. 22, 2717-2721.

120. Foix, D.; Ramis, X.; Sangermano, M.; Serra, A. Synthesis of a New Hyperbranched-LinearHyperbranched Triblock Copolymer and Its Use as a Chemical Modifier for the Cationic Photo and Thermal Curing of Epoxy Resins. J. Polym. Sci. Part A Polym. Chem. 2012, 50 (6), $1133-$ 1142.

121. Galbis, J. A.; García-Martín, M. D. G.; De Paz, M. V.; Galbis, E. Synthetic Polymers from SugarBased Monomers. Chemical Reviews. 2016.

122. Fenouillot, F.; Rousseau, A.; Colomines, G.; Saint-Loup, R.; Pascault, J. P. Polymers from Renewable 1,4:3,6-Dianhydrohexitols (Isosorbide, Isomannide and Isoidide): A Review. Progress in Polymer Science (Oxford). 2010.

123. Caouthar, A.; Roger, P.; Tessier, M.; Chatti, S.; Blais, J. C.; Bortolussi, M. Synthesis and Characterization of New Polyamides Derived from di(4-Cyanophenyl)isosorbide. Eur. Polym. $J$. 2007, 43 (1), 220-230.

124. Thiem, J.; Lüders, H. Darstellung Und Gezielte Polykondensation von Anhydroalditol-Bausteinen Aus Stärke. Starch - Stärke 1984, 36 (5), 170-176.

125. Thiem, J.; Lüders, H. Synthesis and Properties of Polyurethanes Derived from Diaminodianhydroalditols. Die Makromol. Chemie 1986, 187 (12), 2775-2785.

126. Stoss, P.; Hemmer, R. 1,4:3,6-Dianhydrohexitols. In Advances in Carbohydrate Chemistry and Biochemistry; Horton, D., Ed.; Academic Press, 1991; Vol. 49, pp 93-173.

127. Xi, J.; Zhang, Y.; Ding, D.; Xia, Q.; Wang, J.; Liu, X.; Lu, G.; Wang, Y. Catalytic Production of Isosorbide from Cellulose over Mesoporous Niobium Phosphate-Based Heterogeneous Catalysts via a Sequential Process. Appl. Catal. A Gen. 2014, 469, 108-115.

128. Bocqué, M.; Voirin, C.; Lapinte, V.; Caillol, S.; Robin, J.-J. Petro-Based and Bio-Based Plasticizers: Chemical Structures to Plasticizing Properties. J. Polym. Sci. Part A Polym. Chem. 
2016, $54(1), 11-33$.

129. Braun, D.; Bergmann, M. 1,4:3,6-Dianhydrohexite Als Bausteine Für Polymere. J. für Prakt. Chemie/Chemiker-Zeitung 1992, 334 (4), 298-310.

130. Okada, M.; Okada, Y.; Tao, A.; Aoi, K. Biodegradable Polymers Based on Renewable Resources: Polyesters Composed of 1,4:3,6-Dianhydrohexitol and Aliphatic Dicarboxylic Acid Units. $J$. Appl. Polym. Sci. 1996, 59 (1199-1202).

131. Okada, M.; Okada, Y.; Aoi, K. Synthesis and Degradabilities of Polyesters from 1,4:3,6Dianhydrohexitols and Aliphatic Dicarboxylic Acids. J. Polym. Sci. Part A Polym. Chem. 1995, 33 (16), 2813-2820.

132. Storbeck, R.; Rehahn, M.; Ballauff, M. Synthesis and Properties of High-Molecular-Weight Polyesters Based on 1,4:3,6-Dianhydrohexitols and Terephthalic Acid. Die Makromol. Chemie 1993, 194 (1), 53-64.

133. Storbeck, R.; Ballauff, M. Synthesis and Thermal Analysis of Copolyesters Deriving from 1,4:3,6Dianhydrosorbitol, Ethylene Glycol, and Terephthalic Acid. J. Appl. Polym. Sci. 1996, 59, 1199 1202.

134. Storbeck, R.; Ballauff, M. Synthesis and Properties of Polyesters Based on 2,5-Furandicarboxylic Acid and 1,4:3,6-Dianhydrohexitols. Polymer (Guildf). 1993, 34 (23), 5003-5006.

135. Chatti, S.; Schwarz, G.; Kricheldorf, H. R. Cyclic and Noncyclic Polycarbonates of Isosorbide (1,4:3,6-Dianhydro-D-Glucitol). Macromolecules 2006, 39 (26), 9064-9070.

136. Chatti, S.; Kricheldorf, H. R.; Schwarz, G. Copolycarbonates of Isosorbide and Various Diols. $J$. Polym. Sci. Part A Polym. Chem. 2006, 44 (11), 3616-3628.

137. Bachmann, F.; Reimer, J.; Ruppenstein, M.; Thiem, J. Synthesis of Novel Polyurethanes and Polyureas by Polyaddition Reactions of Dianhydrohexitol Configurated Diisocyanates. Macromol. Chem. Phys. 2001.

138. Sangeetha, V. H.; Deka, H.; Varghese, T. O.; Nayak, S. K. State of the Art and Future Prospectives of Poly(lactic Acid) Based Blends and Composites. Polym. Compos. 2018, 39 (1), 81-101.

139. Hofvendahl, K.; Hahn-Hägerdal, B. Factors Affecting the Fermentative Lactic Acid Production from Renewable resources1. Enzyme Microb. Technol. 2000, 26 (2), 87-107.

140. Vink, E. T. H.; Rábago, K. R.; Glassner, D. A.; Gruber, P. R. Applications of Life Cycle Assessment to NatureWorks ${ }^{\mathrm{TM}}$ Polylactide (PLA) Production. Polym. Degrad. Stab. 2003, 80 (3), 403-419.

141. Madhavan Nampoothiri, K.; Nair, N. R.; John, R. P. An Overview of the Recent Developments in Polylactide (PLA) Research. Bioresour. Technol. 2010, 101 (22), 8493-8501.

142. John, R. P.; Nampoothiri, K. M.; Pandey, A. Fermentative Production of Lactic Acid from Biomass: An Overview on Process Developments and Future Perspectives. Appl. Microbiol. Biotechnol. 2007, 74 (3), 524-534.

143. Datta, R.; Henry, M. Lactic Acid: Recent Advances in Products, Processes and Technologies - a Review. J. Chem. Technol. Biotechnol. 2006, 81 (7), 1119-1129.

144. Reddy, G.; Altaf, M.; Naveena, B. J.; Venkateshwar, M.; Kumar, E. V. Amylolytic Bacterial Lactic Acid Fermentation - A Review. Biotechnol. Adv. 2008, 26 (1), 22-34. 
145. Södergård, A.; Stolt, M. Properties of Lactic Acid Based Polymers and Their Correlation with Composition. Prog. Polym. Sci. 2002, 27 (6), 1123-1163.

146. Garlotta, D. A Literature Review of Poly(Lactic Acid). J. Polym. Environ. 2001, 9 (2), 63-84.

147. Lim, L.-T.; Auras, R.; Rubino, M. Processing Technologies for Poly(lactic Acid). Prog. Polym. Sci. 2008, 33 (8), 820-852.

148. Hiltunen, K.; Seppälä, J. V.; Härkönen, M. Lactic Acid Based Poly(ester-Urethanes): Use of Hydroxyl Terminated Prepolymer in Urethane Synthesis. J. Appl. Polym. Sci. 1997, 63, 10911100 .

149. Tuominen, J.; Seppälä, J. V. Synthesis and Characterization of Lactic Acid Based Poly(ester-amide). Macromolecules 2000, 33 (10), 3530-3535.

150. Moon, S.-I.; Lee, C.-W.; Taniguchi, I.; Miyamoto, M.; Kimura, Y. Melt/solid Polycondensation of L-Lactic Acid: An Alternative Route to Poly(1-Lactic Acid) with High Molecular Weight. Polymer (Guildf). 2001, 42 (11), 5059-5062.

151. Penczek, S.; Duda, A.; Szymanski, R.; Biela, T. What We Have Learned in General from Cyclic Esters Polymerization. Macromol. Symp. 2000, 153, 1-15.

152. Dove, A. P. Organic Catalysis for Ring-Opening Polymerization. ACS Macro Lett. 2012, 1 (12), $1409-1412$.

153. Albertsson, A.-C.; Srivastava, R. K. Recent Developments in Enzyme-Catalyzed Ring-Opening Polymerization. Adv. Drug Deliv. Rev. 2008, 60 (9), 1077-1093.

154. Thomas, C. M. Stereocontrolled Ring-Opening Polymerization of Cyclic Esters: Synthesis of New Polyester Microstructures. Chem. Soc. Rev. 2010, 39 (1), 165-173.

155. Dechy-Cabaret, O.; Martin-Vaca, B.; Bourissou, D. Controlled Ring-Opening Polymerization of Lactide and Glycolide. Chem. Rev. 2004, 104 (12), 6147-6176.

156. Jérôme, C.; Lecomte, P. Recent Advances in the Synthesis of Aliphatic Polyesters by RingOpening Polymerization. Adv. Drug Deliv. Rev. 2008, 60 (9), 1056-1076.

157. Kamber, N. E.; Jeong, W.; Waymouth, R. M.; Pratt, R. C.; Lohmeijer, B. G. G.; Hedrick, J. L. Organocatalytic Ring-Opening Polymerization. Chem. Rev. 2007, 107 (12), 5813-5840.

158. Nijenhuis, A. J.; Grijpma, D. W.; Pennings, A. J. Lewis Acid Catalyzed Polymerization of LLactide. Kinetics and Mechanism of the Bulk Polymerization. Macromolecules 1992, 25 (24), 6419-6424.

159. Rasal, R. M.; Janorkar, A. V; Hirt, D. E. Poly(lactic Acid) Modifications. Prog. Polym. Sci. 2010, 35 (3), 338-356.

160. Stanford, M. J.; Dove, A. P. Stereocontrolled Ring-Opening Polymerisation of Lactide. Chem. Soc. Rev. 2010, 39 (2), 486-494.

161. Vasanthakumari, R.; Pennings, A. J. Crystallization Kinetics of Poly(1-Lactic Acid). Polymer (Guildf). 1983, 24 (2), 175-178.

162. Kalb, B.; Pennings, A. J. General Crystallization Behaviour of Poly(1-Lactic Acid). Polymer (Guildf). 1980, 21 (6), 607-612.

163. Ikada, Y.; Jamshidi, K.; Tsuji, H.; Hyon, S. H. Stereocomplex Formation between Enantiomeric Poly(lactides). Macromolecules 1987, 20 (4), 904-906. 
164. Tsuji, H.; Ikada, Y. Stereocomplex Formation between Enantiomeric Poly(lactic Acids). 9. Stereocomplexation from the Melt. Macromolecules 1993, 26 (25), 6918-6926.

165. Tsuji, H. Poly(lactide) Stereocomplexes: Formation, Structure, Properties, Degradation, and Applications. Macromol. Biosci. 2005, 5 (7), 569-597.

166. Hoogsteen, W.; Postema, A. R.; Pennings, A. J.; Ten Brinke, G.; Zugenmaier, P. Crystal Structure, Conformation and Morphology of Solution-Spun poly(L-Lactide) Fibers. Macromolecules 1990, 23 (2), 634-642.

167. Puiggali, J.; Ikada, Y.; Tsuji, H.; Cartier, L.; Okihara, T.; Lotz, B. The Frustrated Structure of Poly(1-Lactide). Polymer (Guildf). 2000, 41 (25), 8921-8930.

168. Cartier, L.; Okihara, T.; Ikada, Y.; Tsuji, H.; Puiggali, J.; Lotz, B. Epitaxial Crystallization and Crystalline Polymorphism of Polylactides. Polymer (Guildf). 2000, 41 (25), 8909-8919.

169. Pan, P.; Inoue, Y. Polymorphism and Isomorphism in Biodegradable Polyesters. Prog. Polym. Sci. 2009, 34 (7), 605-640.

170. Perego, G.; Cella, G. D.; Bastioli, C. Effect of Molecular Weight and Crystallinity on Poly(lactic Acid) Mechanical Properties. J. Appl. Polym. Sci. 1996, 59, 37-43.

171. Auras, R.; Harte, B.; Selke, S. An Overview of Polylactides as Packaging Materials. Macromol. Biosci. 2004, 4 (9), 835-864.

172. Taubner, V.; Shishoo, R. Influence of Processing Parameters on the Degradation of poly(LLactide) during Extrusion. J. Appl. Polym. Sci. 2001, 79, 2128-2135.

173. Kale, G.; Auras, R.; Singh, S. P.; Narayan, R. Biodegradability of Polylactide Bottles in Real and Simulated Composting Conditions. Polym. Test. 2007, 26 (8), 1049-1061.

174. Kale, G.; Kijchavengkul, T.; Auras, R.; Rubino, M.; Selke, S. E.; Singh, S. P. Compostability of Bioplastic Packaging Materials: An Overview. Macromol. Biosci. 2007, 7 (3), 255-277.

175. Park, K. I.; Xanthos, M. A Study on the Degradation of Polylactic Acid in the Presence of Phosphonium Ionic Liquids. Polym. Degrad. Stab. 2009, 94 (5), 834-844.

176. Bordes, P.; Pollet, E.; Avérous, L. Nano-Biocomposites: Biodegradable Polyester/nanoclay Systems. Prog. Polym. Sci. 2009, 34 (2), 125-155.

177. Muzumdar, A. V; Sawant, S. B.; Pangarkar, V. G. Reduction of Maleic Acid to Succinic Acid on Titanium Cathode. Org. Process Res. Dev. 2004, 8 (4), 685-688.

178. Choudhary, H.; Nishimura, S.; Ebitani, K. Metal-Free Oxidative Synthesis of Succinic Acid from Biomass-Derived Furan Compounds Using a Solid Acid Catalyst with Hydrogen Peroxide. Appl. Catal. A Gen. 2013, 458, 55-62.

179. Song, H.; Lee, S. Y. Production of Succinic Acid by Bacterial Fermentation. Enzyme Microb. Technol. 2006, 39 (3), 352-361.

180. Lee, P. C.; Lee, W. G.; Lee, S. Y.; Chang, H. N. Succinic Acid Production with Reduced byProduct Formation in the Fermentation of Anaerobiospirillum Succiniciproducens Using Glycerol as a Carbon Source. Biotechnol. Bioeng. 2001, 72 (1), 41-48.

181. Stempfle, F.; OrTmann, P.; Mecking, S. Long-Chain Aliphatic Polymers To Bridge the Gap between Semicrystalline Polyolefins and Traditional Polycondensates. Chem. Rev. 2016, 116 (7), $4597-4641$.

182. Zhu, C.; Zhang, Z.; Liu, Q.; Wang, Z.; Jin, J. Synthesis and Biodegradation of Aliphatic Polyesters 
from Dicarboxylic Acids and Diols. J. Appl. Polym. Sci. 2003, 90 (4), 982-990.

183. Nikolic, M. S.; Djonlagic, J. Synthesis and Characterization of Biodegradable Poly(butylene Succinate-Co-Butylene Adipate)s. Polym. Degrad. Stab. 2001, 74 (2), 263-270.

184. Bikiaris, D. N.; Papageorgiou, G. Z.; Achilias, D. S. Synthesis and Comparative Biodegradability Studies of Three Poly(alkylene Succinate)s. Polym. Degrad. Stab. 2006, 91 (1), 31-43.

185. Fujimaki, T. Processability and Properties of Aliphatic Polyesters, "BIONOLLE", Synthesized by Polycondensation Reaction. Polym. Degrad. Stab. 1998, 59 (1), 209-214.

186. Shirahama, H.; Kawaguchi, Y.; Aludin, M. S.; Yasuda, H. Synthesis and Enzymatic Degradation of High Molecular Weight Aliphatic Polyesters. J. Appl. Polym. Sci. 2001, 80, 340-347.

187. Wang, Q.; Shao, Z.; Yu, T. The Synthesis and Characterization of Polyethylene Succinamide (Polyamide 24). Polym. Bull. 1996, 36 (6), 659-665.

188. Gaymans, R. J.; Venkatraman, V. S.; Schuijer, J. Preparation and Some Properties of Nylon-4,2. J. Polym. Sci. Polym. Chem. Ed. 1984, 22 (6), 1373-1382.

189. Tokiwa, Y.; Suzuki, T.; Ando, T. Synthesis of Copolyamide-esters and Some Aspects Involved in Their Hydrolysis by Lipase. J. Appl. Polym. Sci. 1979, 24 (7), 1701-1711.

190. Grigat, E.; Koch, R.; Timmermann, R. BAR 1095 and BAK 2195: Completely Biodegradable Synthetic Thermoplastics. Polym. Degrad. Stab. 1998, 59 (1), 223-226.

191. Moore, T.; Adhikari, R.; Gunatillake, P. Chemosynthesis of Bioresorbable Poly( $\gamma$-Butyrolactone) by Ring-Opening Polymerisation: A Review. Biomaterials 2005, 26 (18), 3771-3782.

192. Maisonneuve, L.; Lebarbe, T.; Grau, E.; Cramail, H. Structure-Properties Relationship of Fatty Acid-Based Thermoplastics as Synthetic Polymer Mimics. Polym. Chem. 2013, 4 (22), 54725517.

193. Nagai, K. New Developments in the Production of Methyl Methacrylate. Appl. Catal. A Gen. 2001, 221 (1), 367-377.

194. Willke, T.; Vorlop, K.-D. Biotechnological Production of Itaconic Acid. Appl. Microbiol. Biotechnol. 2001, 56 (3), 289-295.

195. Ishida, S.; Saito, S. Polymerization of Itaconic Acid Derivatives. J. Polym. Sci. Part A-1 Polym. Chem. 1967, 5 (4), 689-705.

196. Watanabe, H.; Matsumoto, A.; Otsu, T. Polymerization of N-Alkyl-Substituted Itaconimides and N-(Alkyl-Substituted Phenyl)itaconimides and Characterization of the Resulting Polymers. $J$. Polym. Sci. Part A Polym. Chem. 1994, 32 (11), 2073-2083.

197. Satoh, K. Controlled/living Polymerization of Renewable Vinyl Monomers into Bio-Based Polymers. Polym. J. 2015, 47, 527.

198. Satoh, K.; Lee, D.-H.; Nagai, K.; Kamigaito, M. Precision Synthesis of Bio-Based Acrylic Thermoplastic Elastomer by RAFT Polymerization of Itaconic Acid Derivatives. Macromol. Rapid Commun. 2014, 35 (2), 161-167.

199. Robert, T.; Friebel, S. Itaconic Acid - a Versatile Building Block for Renewable Polyesters with Enhanced Functionality. Green Chem. 2016, 18 (10), 2922-2934.

200. Farmer, T. J.; Castle, R. L.; Clark, J. H.; Macquarrie, D. J. Synthesis of Unsaturated Polyester Resins from Various Bio-Derived Platform Molecules. Int. J. Mol. Sci. 2015, 16, 14912-14932. 
201. Chanda, S.; Ramakrishnan, S. Poly(alkylene Itaconate)s - an Interesting Class of Polyesters with Periodically Located Exo-Chain Double Bonds Susceptible to Michael Addition. Polym. Chem. 2015, 6 (11), 2108-2114.

202. Ma, S.; Liu, X.; Jiang, Y.; Tang, Z.; Zhang, C.; Zhu, J. Bio-Based Epoxy Resin from Itaconic Acid and Its Thermosets Cured with Anhydride and Comonomers. Green Chem. 2013, 15 (1), 245-254.

203. Winkler, M.; Lacerda, T. M.; Mack, F.; Meier, M. A. R. Renewable Polymers from Itaconic Acid by Polycondensation and Ring-Opening-Metathesis Polymerization. Macromolecules 2015, 48 (5), 1398-1403.

204. Bugnicourt, E.; Cinelli, P.; Lazzeri, A.; Alvarez, V. Polyhydroxyalkanoate (PHA): Review of Synthesis, Characteristics, Processing and Potential Applications in Packaging. Express Polym. Lett. 2014, 8 (11), 791-808.

205. Laycock, B.; Halley, P.; Pratt, S.; Werker, A.; Lant, P. The Chemomechanical Properties of Microbial Polyhydroxyalkanoates. Prog. Polym. Sci. 2013, 38 (3), 536-583.

206. Laurichesse, S.; Avérous, L. Chemical Modification of Lignins: Towards Biobased Polymers. Prog. Polym. Sci. 2014, 39 (7), 1266-1290.

207. Silva, E. A. B. da; Zabkova, M.; Araújo, J. D.; Cateto, C. A.; Barreiro, M. F.; Belgacem, M. N.; Rodrigues, A. E. An Integrated Process to Produce Vanillin and Lignin-Based Polyurethanes from Kraft Lignin. Chem. Eng. Res. Des. 2009, 87 (9), 1276-1292.

208. Hatakeyama, H.; Hirogaki, A.; Matsumura, H.; Hatakeyama, T. Glass Transition Temperature of Polyurethane Foams Derived from Lignin by Controlled Reaction Rate. J. Therm. Anal. Calorim. 2013, 114 (3), 1075-1082.

209. Cinelli, P.; Anguillesi, I.; Lazzeri, A. Green Synthesis of Flexible Polyurethane Foams from Liquefied Lignin. Eur. Polym. J. 2013, 49 (6), 1174-1184.

210. Simionescu, C. I.; Rusan, V.; Macoveanu, M. M.; Cazacu, G.; Lipsa, R.; Vasile, C.; Stoleriu, A.; Ioanid, A. Lignin/epoxy Composites. Compos. Sci. Technol. 1993, 48 (1), 317-323.

211. Feldman, D.; Banu, D.; Natansohn, A.; Wang, J. Structure-properties Relations of Thermally Cured Epoxy-lignin Polyblends. J. Appl. Polym. Sci. 1991, 42 (6), 1537-1550.

212. Feldman, D.; Banu, D.; Luchian, C.; Wang, J. Epoxy-lignin Polyblends: Correlation between Polymer Interaction and Curing Temperature. J. Appl. Polym. Sci. 1991, 42 (5), 1307-1318.

213. Delmas, G.-H.; Benjelloun-Mlayah, B.; Bigot, Y. Le; Delmas, M. Biolignin ${ }^{\mathrm{TM}}$ Based Epoxy Resins. J. Appl. Polym. Sci. 2013, 127 (3), 1863-1872.

214. Hirose, S.; Hatakeyama, T.; Hatakeyama, H. Synthesis and Thermal Properties of Epoxy Resins from Ester-Carboxylic Acid Derivative of Alcoholysis Lignin. Macromol. Symp. 2003, 197 (1), $157-170$.

215. Over, L. C.; Grau, E.; Grelier, S.; Meier, M. A. R.; Cramail, H. Synthesis and Characterization of Epoxy Thermosetting Polymers from Glycidylated Organosolv Lignin and Bisphenol A. Macromol. Chem. Phys. 2017, 218 (4), 1600411-n/a.

216. Muller, P. C.; Kelley, S. S.; Glasser, W. G. Engineering Plastics from Lignin. IX. Phenolic Resin Synthesis and Characterization. J. Adhes. 1984, 17 (3), 185-206.

217. Wang, M.; Leitch, M.; (Charles) Xu, C. Synthesis of Phenol-formaldehyde Resol Resins Using Organosolv Pine Lignins. Eur. Polym. J. 2009, 45 (12), 3380-3388. 
218. Li, J.; Zhang, J.; Zhang, S.; Gao, Q.; Li, J.; Zhang, W. Fast Curing Bio-Based Phenolic Resins via Lignin Demethylated under Mild Reaction Condition. Polymers (Basel). 2017.

219. Sawamura, K.; Tobimatsu, Y.; Kamitakahara, H.; Takano, T. Lignin Functionalization through Chemical Demethylation: Preparation and Tannin-Like Properties of Demethylated Guaiacyl-Type Synthetic Lignins. ACS Sustain. Chem. Eng. 2017, 5 (6), 5424-5431.

220. Ferhan, M.; Yan, N.; Sain, M. A New Method for Demethylation of Lignin from Woody Biomass Using Biophysical Methods. J Chem Eng Process Technol 2013, 4, 160.

221. Zakzeski, J.; Jongerius, A. L.; Bruijnincx, P. C. A.; Weckhuysen, B. M. Catalytic Lignin Valorization Process for the Production of Aromatic Chemicals and Hydrogen. ChemSusChem 2012, 5 (8), 1602-1609.

222. Chen, L.; Xin, J.; Ni, L.; Dong, H.; Yan, D.; Lu, X.; Zhang, S. Conversion of Lignin Model Compounds under Mild Conditions in Pseudo-Homogeneous Systems. Green Chem. 2016, 18 (8), $2341-2352$.

223. Lange, H.; Decina, S.; Crestini, C. Oxidative Upgrade of Lignin - Recent Routes Reviewed. Eur. Polym. J. 2013, 49 (6), 1151-1173.

224. Harms, R. G.; Markovits, I. I. E.; Drees, M.; Herrmann, h. c. mult. W. A.; Cokoja, M.; Kühn, F. E. Cleavage of C O Bonds in Lignin Model Compounds Catalyzed by Methyldioxorhenium in Homogeneous Phase. ChemSusChem 2014, 7 (2), 429-434.

225. Sik Kim, Y.; Chang, H.; Kadla, J. F. Polyoxometalate (POM) Oxidation of Milled Wood Lignin (MWL). J. Wood Chem. Technol. 2007, 27 (3-4), 225-241.

226. Zhao, Y.; Xu, Q.; Pan, T.; Zuo, Y.; Fu, Y.; Guo, Q.-X. Depolymerization of Lignin by Catalytic Oxidation with Aqueous Polyoxometalates. Appl. Catal. A Gen. 2013, 467, 504-508.

227. Crestini, C.; Saladino, R.; Tagliatesta, P.; Boschi, T. Biomimetic Degradation of Lignin and Lignin Model Compounds by Synthetic Anionic and Cationic Water Soluble Manganese and Iron Porphyrins. Bioorg. Med. Chem. 1999, 7 (9), 1897-1905.

228. Rahimi, A.; Ulbrich, A.; Coon, J. J.; Stahl, S. S. Formic-Acid-Induced Depolymerization of Oxidized Lignin to Aromatics. Nature 2014, 515, 249.

229. Llevot, A.; Grau, E.; Carlotti, S.; Grelier, S.; Cramail, H. From Lignin-Derived Aromatic Compounds to Novel Biobased Polymers. Macromol. Rapid Commun. 2016, 37 (1), 9-28.

230. Stanzione III, J. F.; Sadler, J. M.; La Scala, J. J.; Reno, K. H.; Wool, R. P. Vanillin-Based Resin for Use in Composite Applications. Green Chem. 2012, 14 (8), 2346-2352.

231. Meylemans, H. A.; Harvey, B. G.; Reams, J. T.; Guenthner, A. J.; Cambrea, L. R.; Groshens, T. J.; Baldwin, L. C.; Garrison, M. D.; Mabry, J. M. Synthesis, Characterization, and Cure Chemistry of Renewable Bis(cyanate) Esters Derived from 2-Methoxy-4-Methylphenol. Biomacromolecules 2013, 14 (3), 771-780.

232. Fache, M.; Darroman, E.; Besse, V.; Auvergne, R.; Caillol, S.; Boutevin, B. Vanillin, a Promising Biobased Building-Block for Monomer Synthesis. Green Chem. 2014, 16 (4), 1987-1998.

233. Fache, M.; Auvergne, R.; Boutevin, B.; Caillol, S. New Vanillin-Derived Diepoxy Monomers for the Synthesis of Biobased Thermosets. Eur. Polym. J. 2015, 67, 527-538.

234. Fache, M.; Viola, A.; Auvergne, R.; Boutevin, B.; Caillol, S. Biobased Epoxy Thermosets from Vanillin-Derived Oligomers. Eur. Polym. J. 2015, 68, 526-535. 
235. Bock, L. H.; Anderson, J. K. Linear Polyesters Derived from Vanillic Acid. J. Polym. Sci. 1955, 17 (86), 553-558.

236. Lange, W.; Kordsachia, O. Darstellung Und Eigenschaften von Aus Vanillin Und Syringaaldehyd Erhältlichen Polyestern. Holz als Roh- und Werkst. 1981, 39 (3), 107-112.

237. Kricheldorf, H. R.; Löhden, G. Whisker 11. Poly(ester-amide)s Derived from Vanillic Acid and 4-Aminobenzoic Acid. Polymer (Guildf). 1995, 36 (8), 1697-1705.

238. Montes de Oca, H.; Wilson, J. E.; Penrose, A.; Langton, D. M.; Dagger, A. C.; Anderson, M.; Farrar, D. F.; Lovell, C. S.; Ries, M. E.; Ward, I. M.; et al. Liquid-Crystalline Aromatic-aliphatic Copolyester Bioresorbable Polymers. Biomaterials 2010, 31 (30), 7599-7605.

239. Kreye, O.; Oelmann, S.; Meier, M. A. R. Renewable Aromatic-Aliphatic Copolyesters Derived from Rapeseed. Macromol. Chem. Phys. 2013, 214 (13), 1452-1464.

240. Firdaus, M.; Meier, M. A. R. Renewable Co-Polymers Derived from Vanillin and Fatty Acid Derivatives. Eur. Polym. J. 2013, 49 (1), 156-166.

241. Mialon, L.; Pemba, A. G.; Miller, S. A. Biorenewable Polyethylene Terephthalate Mimics Derived from Lignin and Acetic Acid. Green Chem. 2010, 12 (10), 1704-1706.

242. Mialon, L.; Vanderhenst, R.; Pemba, A. G.; Miller, S. A. Polyalkylenehydroxybenzoates (PAHBs): Biorenewable Aromatic/Aliphatic Polyesters from Lignin. Macromol. Rapid Commun. 2011, 32 (17), 1386-1392.

243. Pang, C.; Zhang, J.; Wu, G.; Wang, Y.; Gao, H.; Ma, J. Renewable Polyesters Derived from 10Undecenoic Acid and Vanillic Acid with Versatile Properties. Polym. Chem. 2014, 5 (8), 28432853.

244. Pion, F.; Reano, A. F.; Ducrot, P.-H.; Allais, F. Chemo-Enzymatic Preparation of New Bio-Based Bis- and Trisphenols: New Versatile Building Blocks for Polymer Chemistry. RSC Adv. 2013, 3 (23), 8988-8997.

245. Pion, F.; Ducrot, P.-H.; Allais, F. Renewable Alternating Aliphatic-Aromatic Copolyesters Derived from Biobased Ferulic Acid, Diols, and Diacids: Sustainable Polymers with Tunable Thermal Properties. Macromol. Chem. Phys. 2014, 215 (5), 431-439.

246. Llevot, A.; Grau, E.; Carlotti, S.; Grelier, S.; Cramail, H. Selective Laccase-Catalyzed Dimerization of Phenolic Compounds Derived from Lignin: Towards Original Symmetrical BioBased (Bis) Aromatic Monomers. J. Mol. Catal. B Enzym. 2016, 125, 34-41.

247. Llevot, A.; Grau, E.; Carlotti, S.; Grelier, S.; Cramail, H. Renewable (Semi)aromatic Polyesters from Symmetrical Vanillin-Based Dimers. Polym. Chem. 2015, 6 (33), 6058-6066.

248. Kreye, O.; Tóth, T.; Meier, M. A. R. Copolymers Derived from Rapeseed Derivatives via ADMET and Thiol-Ene Addition. Eur. Polym. J. 2011, 47 (9), 1804-1816.

249. Barbara, I.; Flourat, A. L.; Allais, F. Renewable Polymers Derived from Ferulic Acid and Biobased Diols via ADMET. Eur. Polym. J. 2015, 62, 236-243.

250. Llevot, A.; Grau, E.; Carlotti, S.; Grelier, S.; Cramail, H. ADMET Polymerization of Bio-Based Biphenyl Compounds. Polym. Chem. 2015, 6 (44), 7693-7700.

251. Lebarbé, T.; More, A. S.; Sane, P. S.; Grau, E.; Alfos, C.; Cramail, H. Bio-Based Aliphatic Polyurethanes through ADMET Polymerization in Bulk and Green Solvent. Macromol. Rapid Commun. 2014, 35 (4), 479-483. 
252. Pemba, A. G.; Rostagno, M.; Lee, T. A.; Miller, S. A. Cyclic and Spirocyclic Polyacetal Ethers from Lignin-Based Aromatics. Polym. Chem. 2014, 5 (9), 3214-3221.

253. Harvey, B. G.; Guenthner, A. J.; Meylemans, H. A.; Haines, S. R. L.; Lamison, K. R.; Groshens, T. J.; Cambrea, L. R.; Davis, M. C.; Lai, W. W. Renewable Thermosetting Resins and Thermoplastics from Vanillin. Green Chem. 2015, 17 (2), 1249-1258.

254. Noel, A.; Borguet, Y. P.; Raymond, J. E.; Wooley, K. L. Poly(carbonate-amide)s Derived from Bio-Based Resources: Poly(ferulic Acid-Co-Tyrosine). Macromolecules 2014, 47 (9), 2974-2983.

255. Liu, H.; Lepoittevin, B.; Roddier, C.; Guerineau, V.; Bech, L.; Herry, J.-M.; Bellon-Fontaine, M.N.; Roger, P. Facile Synthesis and Promising Antibacterial Properties of a New Guaiacol-Based Polymer. Polymer (Guildf). 2011, 52 (9), 1908-1916.

256. Holmberg, A. L.; Stanzione, J. F.; Wool, R. P.; Epps, T. H. A Facile Method for Generating Designer Block Copolymers from Functionalized Lignin Model Compounds. ACS Sustain. Chem. Eng. 2014, 2 (4), 569-573.

257. Pfister, D. P.; Xia, Y.; Larock, R. C. Recent Advances in Vegetable Oil-Based Polyurethanes. ChemSusChem 2011, 4 (6), 703-717.

258. Seniha Güner, F.; Yağc1, Y.; Tuncer Erciyes, A. Polymers from Triglyceride Oils. Prog. Polym. Sci. 2006, 31 (7), 633-670.

259. Miao, S.; Wang, P.; Su, Z.; Zhang, S. Vegetable-Oil-Based Polymers as Future Polymeric Biomaterials. Acta Biomater. 2014, 10 (4), 1692-1704.

260. Petrovic, Z. Polyurethanes from Vegetable Oils. Polym. Rev. 2008, 48 (1), 109-155.

261. Desroches, M.; Escouvois, M.; Auvergne, R.; Caillol, S.; Boutevin, B. From Vegetable Oils to Polyurethanes: Synthetic Routes to Polyols and Main Industrial Products. Polym. Rev. 2012, 52 (1), 38-79.

262. Mosiewicki, M. A.; Aranguren, M. I. A Short Review on Novel Biocomposites Based on Plant Oil Precursors. Eur. Polym. J. 2013, 49 (6), 1243-1256.

263. De Espinosa, L.; Meier, M. A. R. Plant Oils: The Perfect Renewable Resource for Polymer Science?! Eur. Polym. J. 2011, 47 (5), 837-852.

264. Lligadas, G.; Ronda, J. C.; Galià, M.; Cádiz, V. Renewable Polymeric Materials from Vegetable Oils: A Perspective. Mater. Today 2013, 16 (9), 337-343.

265. Engels, H. W.; Pirkl, H. G.; Albers, R.; Albach, R. W.; Krause, J.; Hoffmann, A.; Casselmann, H.; Dormish, J. Polyurethanes: Versatile Materials and Sustainable Problem Solvers for Today's Challenges. Angew. Chemie - Int. Ed. 2013, 52 (36), 9422-9441.

266. Maisonneuve, L.; Chollet, G.; Grau, E.; Cramail, H. Vegetable Oils: A Source of Polyols for Polyurethane Materials. OCL 2016, 23 (5), D508.

267. Zhang, C.; Garrison, T. F.; Madbouly, S. A.; Kessler, M. R. Recent Advances in Vegetable OilBased Polymers and Their Composites. Prog. Polym. Sci. 2017.

268. Datta, J.; Głowińska, E. Chemical Modifications of Natural Oils and Examples of Their Usage for Polyurethane Synthesis. J. Elastomers Plast. 2014, 46 (1), 33-42.

269. Alam, M.; Akram, D.; Sharmin, E.; Zafar, F.; Ahmad, S. Vegetable Oil Based Eco-Friendly Coating Materials: A Review Article. Arab. J. Chem. 2014, 7 (4), 469-479.

270. Petrovic, Z. S.; Guo, A.; Zhang, W. Structure and Properties of Polyurethanes Based on 
Halogenated and Nonhalogenated Soy-Polyols. J. Polym. Sci. Part A Polym. Chem. 2000, 38 (22), 4062-4069.

271. Wang, C.-S.; Yang, L.-T.; Ni, B.-L.; Shi, G. Polyurethane Networks from Different Soy-Based Polyols by the Ring Opening of Epoxidized Soybean Oil with Methanol, Glycol, and 1,2Propanediol. J. Appl. Polym. Sci. 2009, 114 (1), 125-131.

272. Zaher, F. A.; El-Mallah, M. H.; El-Hefnawy, M. M. Kinetics of Oxirane Cleavage in Epoxidized Soybean Oil. J. Am. Oil Chem. Soc. 1989, 66 (5), 698-700.

273. Datta, J.; Głowińska, E. Effect of Hydroxylated Soybean Oil and Bio-Based Propanediol on the Structure and Thermal Properties of Synthesized Bio-Polyurethanes. Ind. Crops Prod. 2014, 61, 84-91.

274. Zieleniewska, M.; Auguścik, M.; Prociak, A.; Rojek, P.; Ryszkowska, J. Polyurethane-Urea Substrates from Rapeseed Oil-Based Polyol for Bone Tissue Cultures Intended for Application in Tissue Engineering. Polym. Degrad. Stab. 2014, 108, 241-249.

275. Gaikwad, M. S.; Gite, V. V.; Mahulikar, P. P.; Hundiwale, D. G.; Yemul, O. S. Eco-Friendly Polyurethane Coatings from Cottonseed and Karanja Oil. Prog. Org. Coatings 2015, 86, 164-172.

276. Miao, S.; Zhang, S.; Su, Z.; Wang, P. Synthesis of Bio-Based Polyurethanes from Epoxidized Soybean Oil and Isopropanolamine. J. Appl. Polym. Sci. 2013, 127 (3), 1929-1936.

277. Guo, R.; Ma, C.; Sun, S.; Ma, Y. Kinetic Study on Oxirane Cleavage of Epoxidized Palm Oil. J. Am. Oil Chem. Soc. 2011, 88 (4), 517-521.

278. Tathe, D. S.; Jagtap, R. N. Synthesis of Bio-Based Polyurethane from Modified Prosopis Juliflora Oil. J. Am. Oil Chem. Soc. 2013, 90 (9), 1405-1413.

279. Miao, S.; Callow, N.; Wang, P.; Liu, Y.; Su, Z.; Zhang, S. Soybean Oil-Based Polyurethane Networks: Shape-Memory Effects and Surface Morphologies. J. Am. Oil Chem. Soc. 2013, 90 (9), $1415-1421$.

280. Bakhshi, H.; Yeganeh, H.; Mehdipour-Ataei, S.; Solouk, A.; Irani, S. Polyurethane Coatings Derived from 1,2,3-Triazole-Functionalized Soybean Oil-Based Polyols: Studying Their Physical, Mechanical, Thermal, and Biological Properties. Macromolecules 2013, 46 (19), 7777-7788.

281. Bakhshi, H.; Yeganeh, H.; Mehdipour-Ataei, S. Synthesis and Evaluation of Antibacterial Polyurethane Coatings Made from Soybean Oil Functionalized with Dimethylphenylammonium Iodide and Hydroxyl Groups. J. Biomed. Mater. Res. Part A 2013, 101A (6), 1599-1611.

282. Yari, A.; Yeganeh, H.; Bakhshi, H.; Gharibi, R. Preparation and Characterization of Novel Antibacterial Castor Oil-Based Polyurethane Membranes for Wound Dressing Application. $J$. Biomed. Mater. Res. Part A 2014, 102 (1), 84-96.

283. Kong, X.; Liu, G.; Curtis, J. M. Novel Polyurethane Produced from Canola Oil Based Poly(ether Ester) Polyols: Synthesis, Characterization and Properties. Eur. Polym. J. 2012, 48 (12), $2097-$ 2106.

284. Kong, X.; Liu, G.; Qi, H.; Curtis, J. M. Preparation and Characterization of High-Solid Polyurethane Coating Systems Based on Vegetable Oil Derived Polyols. Prog. Org. Coatings 2013, 76 (9), 1151-1160.

285. Petrović, Z. S.; Cvetković, I.; Hong, D.; Wan, X.; Zhang, W.; Abraham, T. W.; Malsam, J. Vegetable Oil-Based Triols from Hydroformylated Fatty Acids and Polyurethane Elastomers. Eur. J. Lipid Sci. Technol. 2010, 112 (1), 97-102. 
286. Chaudhari, A.; Kulkarni, R.; Mahulikar, P.; Sohn, D.; Gite, V. Development of PU Coatings from Neem Oil Based Alkyds Prepared by the Monoglyceride Route. J. Am. Oil Chem. Soc. 2015, 92 (5), 733-741.

287. Stirna, U.; Fridrihsone, A.; Lazdina, B.; Misāne, M.; Vilsone, D. Biobased Polyurethanes from Rapeseed Oil Polyols: Structure, Mechanical and Thermal Properties. J. Polym. Environ. 2013, 21 (4), 952-962.

288. Stirna, U.; Fridrihsone-Girone, A.; Yakushin, V.; Vilsone, D. Processing and Properties of SprayApplied, 100\% Solids Polyurethane Coatings from Rapeseed Oil Polyols. J. Coatings Technol. Res. 2014, 11 (3), 409-420.

289. Das, B.; Konwar, U.; Mandal, M.; Karak, N. Sunflower Oil Based Biodegradable Hyperbranched Polyurethane as a Thin Film Material. Ind. Crops Prod. 2013, 44, 396-404.

290. Das, S.; Pandey, P.; Mohanty, S.; Nayak, S. K. Influence of NCO/OH and Transesterified Castor Oil on the Structure and Properties of Polyurethane: Synthesis and Characterization. Mater.

Express 2015, 5 (5), 377-389.

291. Guo, A.; Demydov, D.; Zhang, W.; Petrovic, Z. S. Polyols and Polyurethanes from Hydroformylation of Soybean Oil. J. Polym. Environ. 2002, 10 (1/2), 49-52.

292. Vanbesien, T.; Hapiot, F.; Monflier, E. Hydroformylation of Vegetable Oils and the Potential Use of Hydroformylated Fatty Acids. Lipid Technol. 2013, 25 (8), 175-178.

293. Petrović, Z. S.; Guo, A.; Javni, I.; Cvetković, I.; Hong, D. P. Polyurethane Networks from Polyols Obtained by Hydroformylation of Soybean Oil. Polym. Int. 2008, 57 (2), 275-281.

294. Zhang, C.; Ding, R.; Kessler, M. R. Reduction of Epoxidized Vegetable Oils: A Novel Method to Prepare Bio-Based Polyols for Polyurethanes. Macromol. Rapid Commun. 2014, 35 (11), 10681074.

295. Bantchev, G. B.; Kenar, J. A.; Biresaw, G.; Han, M. G. Free Radical Addition of Butanethiol to Vegetable Oil Double Bonds. J. Agric. Food Chem. 2009, 57 (4), 1282-1290.

296. Hoyle, C. E.; Bowman, C. N. Thiol-Ene Click Chemistry. Angew. Chemie Int. Ed. 2010, 49 (9), $1540-1573$.

297. Ionescu, M.; Radojčić, D.; Wan, X.; Petrović, Z. S.; Upshaw, T. A. Functionalized Vegetable Oils as Precursors for Polymers by Thiol-Ene Reaction. Eur. Polym. J. 2015, 67, 439-448.

298. Desroches, M.; Caillol, S.; Lapinte, V.; Auvergne, R.; Boutevin, B. Synthesis of Biobased Polyols by Thiol-Ene Coupling from Vegetable Oils. Macromolecules 2011, 44 (8), 2489-2500.

299. Caillol, S.; Desroches, M.; Carlotti, S.; Auvergne, R.; Boutevin, B. Synthesis of New Polyurethanes from Vegetable Oil by Thiol-Ene Coupling. Green Mater. 2013, 1 (1), 16-26.

300. Lowe, A. Thiol-Ene Click Reactions and Recent Applications in Polymer Materials Synthesis. Polym Chem 2010, 1, 17-36.

301. Alagi, P.; Choi, Y. J.; Hong, S. C. Preparation of Vegetable Oil-Based Polyols with Controlled Hydroxyl Functionalities for Thermoplastic Polyurethane. Eur. Polym. J. 2016, 78, 46-60.

302. Darroman, E.; Auvergne, R.; Boutevin, B.; Caillol, S. New Aromatic Amine Based on Cardanol Giving New Biobased Epoxy Networks with Cardanol. Eur. J. Lipid Sci. Technol. 2014, 116 (2), 178-189.

303. Darroman, E.; Bonnot, L.; Auvergne, R.; Boutevin, B.; Caillol, S. New Aromatic Amine Based on 
Cardanol Giving New Biobased Epoxy Networks with Cardanol. Eur. J. Lipid Sci. Technol. 2015, $117(2), 178-189$.

304. Ionescu, M.; Radojčić, D.; Wan, X.; Shrestha, M. L.; Petrović, Z. S.; Upshaw, T. A. Highly Functional Polyols from Castor Oil for Rigid Polyurethanes. Eur. Polym. J. 2016, 84, 736-749.

305. Poussard, L.; Mariage, J.; Grignard, B.; Detrembleur, C.; Jérôme, C.; Calberg, C.; Heinrichs, B.; De Winter, J.; Gerbaux, P.; Raquez, J.-M.; et al. Non-Isocyanate Polyurethanes from Carbonated Soybean Oil Using Monomeric or Oligomeric Diamines To Achieve Thermosets or Thermoplastics. Macromolecules 2016, 49 (6), 2162-2171.

306. Raquez, J.-M.; Deléglise, M.; Lacrampe, M.-F.; Krawczak, P. Thermosetting (Bio)materials Derived from Renewable Resources: A Critical Review. Prog. Polym. Sci. 2010, 35 (4), 487-509.

307. Zhang, C.; Madbouly, S. A.; Kessler, M. R. Biobased Polyurethanes Prepared from Different Vegetable Oils. ACS Appl. Mater. Interfaces 2015, 7 (2), 1226-1233.

308. Can, E.; Wool, R. P.; Küsefoğlu, S. Soybean- and Castor-Oil-Based Thermosetting Polymers: Mechanical Properties. J. Appl. Polym. Sci. 2006, 102 (2), 1497-1504.

309. Samper, M. D.; Fombuena, V.; Boronat, T.; García-Sanoguera, D.; Balart, R. Thermal and Mechanical Characterization of Epoxy Resins (ELO and ESO) Cured with Anhydrides. J. Am. Oil Chem. Soc. 2012.

310. Espinoza-Perez, J. D.; Nerenz, B. A.; Haagenson, D. M.; Chen, Z.; Ulven, C. A.; Wiesenborn, D. P. Comparison of Curing Agents for Epoxidized Vegetable Oils Applied to Composites. Polym. Compos. 2011, 32 (11), 1806-1816.

311. Farias, M.; Martinelli, M.; Bottega, D. P. Epoxidation of Soybean Oil Using a Homogeneous Catalytic System Based on a Molybdenum (VI) Complex. Appl. Catal. A Gen. 2010, 384 (1-2), 213-219.

312. Farias, M.; Martinelli, M.; Rolim, G. K. Immobilized Molybdenum Acetylacetonate Complex on MonTmorillonite K-10 as Catalyst for Epoxidation of Vegetable Oils. Appl. Catal. A Gen. 2011, $403(1-2), 119-127$.

313. Rüsch gen. Klaas, M.; Warwel, S. Complete and Partial Epoxidation of Plant Oils by LipaseCatalyzed Perhydrolysis. Ind. Crops Prod. 1999, 9 (2), 125-132.

314. Seniha Güner, F.; Yağc1, Y.; Tuncer Erciyes, A. Polymers from Triglyceride Oils. Prog. Polym. Sci. 2006, 31 (7), 633-670.

315. Metzger, J. O. Fats and Oils as Renewable Feedstock for Chemistry. Eur. J. Lipid Sci. Technol. 2009, 111 (9), 865-876.

316. Park, S.-J.; Jin, F.-L.; Lee, J.-R. Synthesis and Thermal Properties of Epoxidized Vegetable Oil. Macromol. Rapid Commun. 2004, 25 (6), 724-727.

317. Liu, Z.; Erhan, S. Z. Ring-Opening Polymerization of Epoxidized Soybean Oil. J. Am. Oil Chem. Soc. 2010, 87 (4), 437-444.

318. Stemmelen, M.; Lapinte, V.; Habas, J.; Robin, J. Plant Oil-Based Epoxy Resins from Fatty Diamines and Epoxidized Vegetable Oil. 2015, 68, 536-545.

319. Stemmelen, M.; Pessel, F.; Lapinte, V.; Caillol, S.; Habas, J.-P.; Robin, J.-J. A Fully Biobased Epoxy Resin from Vegetable Oils: From the Synthesis of the Precursors by Thiol-Ene Reaction to the Study of the Final Material. J. Polym. Sci. Part A Polym. Chem. 2011, 49 (11), 2434-2444. 
320. Czub, Pi.; Franek, I. Epoxy Resins Modified with Palm Oil Derivatives - Preparation and Properties. Polimery 2013, 58 (2), 135-139.

321. Czub, P. Application of Modified Natural Oils as Reactive Diluents for Epoxy Resins. Macromol. Symp. 2006, 242 (1), 60-64.

322. Yang, G.; Rohde, B. J.; Robertson, M. L. Hydrolytic Degradation and Thermal Properties of Epoxy Resins Derived from Soybean Oil. Green Mater. 2013, 1 (2), 125-134.

323. Mashouf Roudsari, G.; Mohanty, A. K.; Misra, M. Study of the Curing Kinetics of Epoxy Resins with Biobased Hardener and Epoxidized Soybean Oil. ACS Sustain. Chem. Eng. 2014, 2 (9), 2111-2116.

324. Altuna, F. I.; Pettarin, V.; Williams, R. J. J. Self-Healable Polymer Networks Based on the CrossLinking of Epoxidised Soybean Oil by an Aqueous Citric Acid Solution. Green Chem. 2013, 15 (12), 3360 .

325. Supanchaiyamat, N.; Shuttleworth, P. S.; Hunt, A. J.; Clark, J. H.; Matharu, A. S. Thermosetting Resin Based on Epoxidised Linseed Oil and Bio-Derived Crosslinker. Green Chem. 2012, 14 (6), 1759 .

326. Omonov, T. S.; Curtis, J. M. Biobased Epoxy Resin from Canola Oil. J. Appl. Polym. Sci. 2014, $131(8)$, n/a-n/a.

327. Omonov, T.; Curtis, J. Aldehyde Free Thermoset Bioresins and Biocomposites. WO2014075182A1, 2014.

328. Tan, S. G.; Ahmad, Z.; Chow, W. S. Relationships of Cure Kinetics and Processing for Epoxidized Soybean Oil Bio-Thermoset. Ind. Crops Prod. 2013, 43, 378-385.

329. Altuna, F. I.; Espósito, L. H.; Ruseckaite, R. A.; Stefani, P. M. Thermal and Mechanical Properties of Anhydride-Cured Epoxy Resins with Different Contents of Biobased Epoxidized Soybean Oil. J. Appl. Polym. Sci. 2011, 120 (2), 789-798.

330. Chen, Y.; Yang, L.; Wu, J.; Ma, L.; Finlow, D. E.; Lin, S.; Song, K. Thermal and Mechanical Properties of Epoxy Resin Toughened with Epoxidized Soybean Oil. J. Therm. Anal. Calorim. 2013, 113 (2), 939-945.

331. Carbonell-Verdu, A.; Bernardi, L.; Garcia-Garcia, D.; Sanchez-Nacher, L.; Balart, R. Development of Environmentally Friendly Composite Matrices from Epoxidized Cottonseed Oil. Eur. Polym. J. 2015, 63, 1-10.

332. Mallégol, J.; Lemaire, J.; Gardette, J.-L. Drier Influence on the Curing of Linseed Oil. Prog. Org. Coatings 2000, 39 (2), 107-113.

333. Hess, P. S.; O’Hare, G. A. Oxidation of Linseed Oil. Ind. Eng. Chem. 1950, 42 (7), 1424-1431.

334. Tallman, K. A.; Roschek, B.; Porter, N. A. Factors Influencing the Autoxidation of Fatty Acids: Effect of Olefin Geometry of the Nonconjugated Diene. J. Am. Chem. Soc. 2004, 126 (30), 92409247.

335. Taylor, W. L. Blowing Drying Oils. J. Am. Oil Chem. Soc. 1950, 27 (11), 472-476.

336. Çakmakl1, B.; Hazer, B.; Tekin, İ. Ö.; Cömert, F. B. Synthesis and Characterization of Polymeric Soybean Oil- G -Methyl Methacrylate (and N -Butyl Methacrylate) Graft Copolymers: Biocompatibility and Bacterial Adhesion. Biomacromolecules 2005, 6 (3), 1750-1758.

337. Keleş, E.; Hazer, B. Autooxidized Polyunsaturated Oils/Oily Acids: Post-It Applications and 
Reactions with Fe(III) and Adhesion Properties. Macromol. Symp. 2008, 269 (1), 154-160.

338. All1, A.; Hazer, B. Poly(N-Isopropylacrylamide) Thermoresponsive Cross-Linked Conjugates Containing Polymeric Soybean Oil And/or Polypropylene Glycol. Eur. Polym. J. 2008, 44 (6), $1701-1713$.

339. Çakmaklı, B.; Hazer, B.; Açıkgöz, Ş.; Can, M.; Cömert, F. B. PMMA-Multigraft Copolymers Derived from Linseed Oil, Soybean Oil, and Linoleic Acid: Protein Adsorption and Bacterial Adherence. J. Appl. Polym. Sci. 2007, 105 (6), 3448-3457.

340. Cakmakli, B.; Hazer, B.; Tekin, I. O.; Kizgut, S.; Koksal, M.; Menceloglu, Y. Synthesis and Characterization of Polymeric Linseed Oil Grafted Methyl Methacrylate or Styrene. Macromol. Biosci. 2004, 4 (7), 649-655.

341. Fengkui, L.; Larock, R. C. Synthesis, Structure and Properties of New Tung Oil-Styrene-Divinylbenzene Copolymers Prepared by Thermal Polymerization. Biomacromolecules 2003.

342. Gabel, A. R.; Stoesser, S. M. Styrene-Tung Oil Copolymer, US2190906 A. 1939.

343. Krompiec, S.; Suwiński, J.; Majewski, J.; Grobelny, J. Isomerization of Vegetable Oils Catalyzed by Ruthenium Complexes. Polish J. Appl. Chem. 1998, Vol. 42 (1), 43-48.

344. Henna, P. H.; Andjelkovic, D. D.; Kundu, P. P.; Larock, R. C. Biobased Thermosets from the Free-Radical Copolymerization of Conjugated Linseed Oil. J. Appl. Polym. Sci. 2007, 104 (2), 979-985.

345. Valverde, M.; Andjelkovic, D.; Kundu, P. P.; Larock, R. C. Conjugated Low-Saturation Soybean Oil Thermosets: Free-Radical Copolymerization with Dicyclopentadiene and Divinylbenzene. $J$. Appl. Polym. Sci. 2008, 107 (1), 423-430.

346. Pelletier, H.; Belgacem, N.; Gandini, A. Acrylated Vegetable Oils as Photocrosslinkable Materials. J. Appl. Polym. Sci. 2006, 99 (6), 3218-3221.

347. La Scala, J.; Wool, R. P. Property Analysis of Triglyceride-Based Thermosets. Polymer (Guildf). 2005, $46(1), 61-69$.

348. Luo, Q.; Liu, M.; Xu, Y. (Frank); Ionescu, M.; Petrović, Z. S. Thermosetting Allyl Resins Derived from Soybean Oil. Macromolecules 2011, 44 (18), 7149-7157.

349. Bunker, S. P.; Wool, R. P. Synthesis and Characterization of Monomers and Polymers for Adhesives from Methyl Oleate. J. Polym. Sci. Part A Polym. Chem. 2002, 40 (4), 451-458.

350. Lu, J.; Khot, S.; Wool, R. P. New Sheet Molding Compound Resins from Soybean Oil. I. Synthesis and Characterization. Polymer (Guildf). 2005, 46 (1), 71-80.

351. Zhan, M.; Wool, R. P. Biobased Composite Resins Design for Electronic Materials. J. Appl. Polym. Sci. 2010, 118 (6), 3274-3283.

352. Zhang, P.; Zhang, J. One-Step Acrylation of Soybean Oil (SO) for the Preparation of SO-Based Macromonomers; 2013; Vol. 15.

353. Ahn, B. K.; Sung, J.; Rahmani, N.; Wang, G.; Kim, N.; Lease, K.; Sun, X. S. UV-Curable, HighShear Pressure-Sensitive Adhesives Derived from Acrylated Epoxidized Soybean Oil. J. Adhes. 2013, 89 (4), 323-338.

354. Li, Y.; Sun, X. S. Synthesis and Characterization of Acrylic Polyols and Polymers from Soybean Oils for Pressure-Sensitive Adhesives. RSC Adv. 2015, 5 (55), 44009-44017. 
355. Çolak, S.; Küsefoğlu, S. H. Synthesis and Interfacial Properties of Aminosilane Derivative of Acrylated Epoxidized Soybean Oil. J. Appl. Polym. Sci. 2007, 104 (4), 2244-2253.

356. Pashley, R. M.; Senden, T. J.; Morris, R. A.; Guthrie, J. T.; He, W. D. Polymerizable Porphyrins. US 5360880A, 1994.

357. Andjelkovic, D. D.; Valverde, M.; Henna, P.; Li, F.; Larock, R. C. Novel Thermosets Prepared by Cationic Copolymerization of Various Vegetable Oils - synthesis and Their Structure-property Relationships. Polymer (Guildf). 2005, 46 (23), 9674-9685.

358. Li, F.; Larock, R. C. New Soybean Oil-Styrene-Divinylbenzene Thermosetting Copolymers. I. Synthesis and Characterization. J. Appl. Polym. Sci. 2001, 80 (4), 658-670.

359. Li, F.; Larock, R. C. New Soybean Oil-Styrene-Divinylbenzene Thermosetting copolymers?IV. Good Damping Properties. Polym. Adv. Technol. 2002, 13 (6), 436-449.

360. Li, F.; Larock, R. C. New Soybean Oil-Styrene-Divinylbenzene Thermosetting Copolymers. II. Dynamic Mechanical Properties. J. Polym. Sci. Part B Polym. Phys. 2000, 38 (21), 2721-2738.

361. Li, F.; Larock, R. C. New Soybean Oil-Styrene-Divinylbenzene Thermosetting Copolymers. III. Tensile Stress-Strain Behavior. J. Polym. Sci. Part B Polym. Phys. 2001, 39 (1), 60-77.

362. Lu, Y.; Larock, R. C. Fabrication, Morphology and Properties of Soybean Oil-Based Composites Reinforced with Continuous Glass Fibers. Macromol. Mater. Eng. 2007, 292 (10-11), 1085-1094.

363. Andjelkovic, D. D.; Larock, R. C. Novel Rubbers from Cationic Copolymerization of Soybean Oils and Dicyclopentadiene. 1. Synthesis and Characterization. 2006.

364. Andjelkovic, D. D.; Lu, Y.; Kessler, M. R.; Larock, R. C. Novel Rubbers from the Cationic Copolymerization of Soybean Oils and Dicyclopentadiene, 2 - Mechanical and Damping Properties. Macromol. Mater. Eng. 2009, 294 (8), 472-483.

365. Liu, Z.; Sharma, B. K.; Erhan, S. Z. From Oligomers to Molecular Giants of Soybean Oil in Supercritical Carbon Dioxide Medium: 1. Preparation of Polymers with Lower Molecular Weight from Soybean Oil. Biomacromolecules 2007, 8 (1), 233-239.

366. Li, F.; Larock, R. C. Thermosetting Polymers from Cationic Copolymerization of Tung Oil: Synthesis and Characterization. J. Appl. Polym. Sci. 2000, 78 (5), 1044-1056.

367. Jin, F.-L.; Park, S.-J. Thermomechanical Behavior of Epoxy Resins Modified with Epoxidized Vegetable Oils. Polym. Int. 2008, 57 (4), 577-583.

368. Jin, F.-L.; Park, S.-J. Impact-Strength Improvement of Epoxy Resins Reinforced with a Biodegradable Polymer. Mater. Sci. Eng. A 2008, 478 (1-2), 402-405.

369. Park, S.-J.; Jin, F.-L.; Lee, J.-R.; Shin, J.-S. Cationic Polymerization and Physicochemical Properties of a Biobased Epoxy Resin Initiated by Thermally Latent Catalysts. Eur. Polym. J. 2005, 41 (2), 231-237.

370. Park, S.-J.; Jin, F.-L.; Lee, J.-R. Effect of Biodegradable Epoxidized Castor Oil on Physicochemical and Mechanical Properties of Epoxy Resins. Macromol. Chem. Phys. 2004, 205 (15), 2048-2054.

371. Kim, M. S.; Lee, K. W.; Endo, T.; Lee, S. B. Benzylpyrazinium Salts as Thermally Latent Initiators in the Polymerization of Glycidyl Phenyl Ether: Substituent Effect on the Initiator Activity and Mechanistic Aspects. Macromolecules 2004, 37 (15), 5830-5834.

372. Refvik, M. D.; Larock, R. C.; Tian, Q. Ruthenium-Catalyzed Metathesis of Vegetable Oils. J. Am. 
Oil Chem. Soc. 1999, 76 (1), 93-98.

373. Refvik, M. D.; Larock, R. C. The Chemistry of Metathesized Soybean Oil. J. Am. Oil Chem. Soc. 1999, $76(1), 99-102$.

374. Tian, Q.; Larock, R. C. Model Studies and the ADMET Polymerization of Soybean Oil. J. Am. Oil Chem. Soc. 2002, 79 (5), 479-488.

375. Henna, P. H.; Larock, R. C. Rubbery Thermosets by Ring-Opening Metathesis Polymerization of a Functionalized Castor Oil and Cyclooctene. Macromol. Mater. Eng. 2007, 292 (12), 1201-1209.

376. Mauldin, T. C.; Haman, K.; Sheng, X.; Henna, P.; Larock, R. C.; Kessler, M. R. Ring-Opening Metathesis Polymerization of a Modified Linseed Oil with Varying Levels of Crosslinking. $J$. Polym. Sci. Part A Polym. Chem. 2008, 46 (20), 6851-6860.

377. Hong, J.; Luo, Q.; Wan, X.; Petrović, Z. S.; Shah, B. K. Biopolymers from Vegetable Oils via Catalyst- and Solvent-Free "Click" Chemistry: Effects of Cross-Linking Density.

Biomacromolecules 2012, 13 (1), 261-266.

378. Hong, J.; Shah, B. K.; Petrović, Z. S. Vegetable Oil Cast Resins via Click Chemistry: Effects of Cross-Linkers. Eur. J. Lipid Sci. Technol. 2013, 115 (1), 55-60.

379. Shibata, M.; Teramoto, N.; Nakamura, Y. High Performance Bio-Based Thermosetting Resins Composed of Tung Oil and Bismaleimide. J. Appl. Polym. Sci. 2011, 119 (2), 896-901.

380. Lacerda, T. M.; Carvalho, A. J. F.; Gandini, A. Two Alternative Approaches to the Diels-Alder Polymerization of Tung Oil. RSC Adv. 2014, 4 (51), 26829.

381. Gandini, A.; Lacerda, T. M. From Monomers to Polymers from Renewable Resources: Recent Advances. Prog. Polym. Sci. 2015.

382. Gandini, A.; Lacerda, T. M.; Carvalho, A. J. F.; Trovatti, E. Progress of Polymers from Renewable Resources: Furans, Vegetable Oils, and Polysaccharides. Chemical Reviews. 2016.

383. Weissermel, K.; Arpe, H.-J. Industrial Organic Chemistry, Fourth Edition; WILEY-VCH: Weinheim, Ed.; Germany, 2013.

384. Haouet, A.; Sepulchre, M.; Spassky, N. Preparation et Proprietes Des poly(R)-Glycidols. Eur. Polym. J. 1983, 19 (12), 1089-1098.

385. Sunder, A.; Hanselmann, R.; Frey, H.; Mülhaupt, R. Controlled Synthesis of Hyperbranched Polyglycerols by Ring-Opening Multibranching Polymerization. Macromolecules 1999, 32 (13), 4240-4246.

386. Sunder, A.; Krämer, M.; Hanselmann, R.; Mülhaupt, R.; Frey, H. Molecular Nanocapsules Based on Amphiphilic Hyperbranched Polyglycerols. Angew. Chemie Int. Ed. 1999, 38, 3552-3555.

387. Wilms, D.; Stiriba, S.-E.; Frey, H. Hyperbranched Polyglycerols: From the Controlled Synthesis of Biocompatible Polyether Polyols to Multipurpose Applications. Acc. Chem. Res. 2010, 43 (1), $129-141$.

388. Cesteros, L. C. A Simple and Green Procedure to Prepare Poly(ethylene Glycol) Networks: Synthesis and Properties. Green Chem. 2011, 13 (1), 197-206.

389. Cauwet, D.; Dubief, C. Cosmetic Composition Containing a Surfactant such as an Alkylpolyglycoside And/or Polyglycerol and an Urethanpolyether. Patent EP 0555155, 1993.

390. Kunieda, H.; Akahane, A.; Jin-Feng; Ishitobi, M. Phase Behavior of Polyglycerol Didodecanoates in Water. J. Colloid Interface Sci. 2002, 245 (2), 365-370. 
391. Morlat, S.; Cezard, N.; Loubinoux, B.; Philippart, J.-L.; Gardette, J.-L. Mechanisms of Photooxidation of Polyglycerol. Polym. Degrad. Stab. 2001, 72 (2), 199-208.

392. Charles, G.; Clacens, J.-M.; Pouilloux, Y.; Barrault, J. Préparation de Diglycérol et Triglycérol Par Polymérisation Directe Du Glycérol En Présence de Catalyseurs Mésoporeux Basiques. OCL 2003, 10 (1), 74-82.

393. Calderón, M.; Quadir, M. A.; Sharma, S. K.; Haag, R. Dendritic Polyglycerols for Biomedical Applications. Adv. Mater. 2010, 22 (2), 190-218.

394. Frey, H.; Haag, R. Dendritic Polyglycerol: A New Versatile Biocompatible Material. Rev. Mol. Biotechnol. 2002, 90 (3), 257-267.

395. Sutter, M.; Da Silva, E.; Duguet, N.; Raoul, Y.; Métay, E.; Lemaire, M. Glycerol Ether Synthesis: A Bench Test for Green Chemistry Concepts and Technologies. Chemical Reviews. 2015.

396. Martin, A.; Richter, M. Oligomerization of Glycerol - a Critical Review. Eur. J. Lipid Sci. Technol. 2011, 113 (1), 100-117.

397. Garti, N.; Aserin, A.; Zaidman, B. Polyglycerol Esters: Optimization and Techno-Economic Evaluation. J. Am. Oil Chem. Soc. 1981, 58 (9), 878-883.

398. Iaych, K.; Dumarçay, S.; Fredon, E.; Gérardin, C.; Lemor, A.; Gérardin, P. Microwave-Assisted Synthesis of Polyglycerol from Glycerol Carbonate. J. Appl. Polym. Sci. 2011, 120 (4), 2354 2360 .

399. Macierzanka, A.; Szeląg, H. Esterification Kinetics of Glycerol with Fatty Acids in the Presence of Zinc Carboxylates: Preparation of Modified Acylglycerol Emulsifiers. Ind. Eng. Chem. Res. 2004, 43 (24), 7744-7753.

400. Detergents and Detergency. In Bailey's Industrial Oil and Fat Products; John Wiley \& Sons, Inc., 2005.

401. Johnson, D. T.; Taconi, K. A. The Glycerin Glut: Options for the Value-Added Conversion of Crude Glycerol Resulting from Biodiesel Production. Environ. Prog. 2007, 26 (4), 338-348.

402. Wyatt, V. T.; Strahan, G. D. Degree of Branching in Hyperbranched Poly(glycerol-Co-Diacid)s Synthesized in Toluene. Polymers (Basel). 2012, 4 (1), 396-407.

403. Kulshrestha, A. S.; Gao, W.; Gross, R. A. Glycerol Copolyesters: Control of Branching and Molecular Weight Using a Lipase Catalyst. Macromolecules 2005, 38 (8), 3193-3204.

404. Vilela, C.; Sousa, A. F.; Fonseca, A. C.; Serra, A. C.; Coelho, J. F. J.; Freire, C. S. R.; Silvestre, A. J. D.; Heise, A.; Benthem, R. A. T. M. van; Péter, F.; et al. The Quest for Sustainable Polyesters Insights into the Future. Polym. Chem. 2014, 5 (9), 3119-3141.

405. Rai, R.; Tallawi, M.; Grigore, A.; Boccaccini, A. R. Synthesis, Properties and Biomedical Applications of Poly(glycerol Sebacate) (PGS): A Review. Prog. Polym. Sci. 2012, 37 (8), 10511078.

406. Halpern, J. M.; Urbanski, R.; Weinstock, A. K.; Iwig, D. F.; Mathers, R. T.; von Recum, H. A. A Biodegradable Thermoset Polymer Made by Esterification of Citric Acid and Glycerol. J. Biomed. Mater. Res. Part A 2014, 102 (5), 1467-1477.

407. Tisserat, B.; O’kuru, R. H.; Hwang, H.; Mohamed, A. A.; Holser, R. Glycerol Citrate Polyesters Produced through Heating without Catalysis. J. Appl. Polym. Sci. 2012, 125 (5), 3429-3437.

408. Parzuchowski, P. G.; Grabowska, M.; Jaroch, M.; Kusznerczuk, M. Synthesis and 
Characterization of Hyperbranched Polyesters from Glycerol-Based AB2 Monomer. J. Polym. Sci. Part A Polym. Chem. 2009, 47 (15), 3860-3868.

409. Zhao, X.; Liu, L.; Dai, H.; Ma, C.; Tan, X.; Yu, R. Synthesis and Application of Water-Soluble Hyperbranched Poly(ester)s from Maleic Anhydride and Glycerol. J. Appl. Polym. Sci. 2009, 113 (5), 3376-3381.

410. Zhou, C.-H. C.; Beltramini, J. N.; Fan, Y.-X.; Lu, G. Q. M. Chemoselective Catalytic Conversion of Glycerol as a Biorenewable Source to Valuable Commodity Chemicals. Chem. Soc. Rev. 2008, 37 (3), 527-549.

411. Yang, Y.; Lu, W.; Cai, J.; Hou, Y.; Ouyang, S.; Xie, W.; Gross, R. A. Poly(oleic Diacid-CoGlycerol): Comparison of Polymer Structure Resulting from Chemical and Lipase Catalysis. Macromolecules 2011, 44 (7), 1977-1985.

412. Uyama, H.; Kuwabara, M.; Tsujimoto, T.; Kobayashi, S. Enzymatic Synthesis and Curing of Biodegradable Epoxide-Containing Polyesters from Renewable Resources. Biomacromolecules 2003, 4 (2), 211-215.

413. Tsujimoto, T.; Uyama, H.; Kobayashi, S. Enzymatic Synthesis of Cross-Linkable Polyesters from Renewable Resources. Biomacromolecules 2001, 2 (1), 29-31.

414. Li, C.; Luo, X.; Li, T.; Tong, X.; Li, Y. Polyurethane Foams Based on Crude Glycerol-Derived Biopolyols: One-Pot Preparation of Biopolyols with Branched Fatty Acid Ester Chains and Its Effects on Foam Formation and Properties. Polym. (United Kingdom) 2014.

415. Piszczyk, Ł.; Strankowski, M.; Danowska, M.; Hejna, A.; Haponiuk, J. T. Rigid Polyurethane Foams from a Polyglycerol-Based Polyol. Eur. Polym. J. 2014.

416. Hu, S.; Luo, X.; Li, Y. Production of Polyols and Waterborne Polyurethane Dispersions from Biodiesel-Derived Crude Glycerol. J. Appl. Polym. Sci. 2015.

417. Gama, N. V.; Silva, R.; Costa, M.; Barros-Timmons, A.; Ferreira, A. Statistical Evaluation of the Effect of Formulation on the Properties of Crude Glycerol Polyurethane Foams. Polym. Test. 2016.

418. Maisonneuve, L.; Lamarzelle, O.; Rix, E.; Grau, E.; Cramail, H. Isocyanate-Free Routes to Polyurethanes and Poly(hydroxy Urethane)s. Chem. Rev. 2015, 115 (22), 12407-12439.

419. Auvergne, R.; Caillol, S.; David, G.; Boutevin, B.; Pascault, J. P. Biobased Thermosetting Epoxy: Present and Future. Chemical Reviews. 2014.

420. Grunchard, F. Process for the Manufacture of Epichlorohydrin. EP 561441, 1993.

421. Tuck, C. O.; Pérez, E.; Horváth, I. T.; Sheldon, R. A.; Poliakoff, M. Valorization of Biomass: Deriving More Value from Waste. Science (80-. ). 2012, 337 (6095), 695.

422. Barua, S.; Dutta, G.; Karak, N. Glycerol Based Tough Hyperbranched Epoxy: Synthesis, Statistical Optimization and Property Evaluation. Chem. Eng. Sci. 2013, 95, 138-147.

423. Shibata, M.; Nakai, K. Preparation and Properties of Biocomposites Composed of Bio-Based Epoxy Resin, Tannic Acid, and Microfibrillated Cellulose. J. Polym. Sci. Part B Polym. Phys. 2010, 48 (4), 425-433.

424. Takada, Y.; Shinbo, K.; Someya, Y.; Shibata, M. Preparation and Properties of Bio-Based Epoxy Montomorillonite Nanocomposites Derived from Polyglycerol Polyglycidyl Ether and $\varepsilon$ Polylysine. J. Appl. Polym. Sci. 2009, 113 (1), 479-484. 
425. Herzog, B.; Kohan, M.; Mestemacher, S.; Pagilagan, R. Polyamides. Ullmann's Encyclopedia of Industrial Chemistry, Wiley-VCH:; 2000.

426. Rogers, M. E.; Long, T. E. Synthetic Methods in Step-Growth Polymers; Wiley-Interscience, 2003.

427. Marchildon, K. Polyamides - Still Strong After Seventy Years. Macromol. React. Eng. 2011, 5 (1), $22-54$.

428. Schmitz, K.; Schepers, U. Polyamides as Artificial Transcription Factors: Novel Tools for Molecular Medicine? Angew. Chemie Int. Ed. 2004, 43 (19), 2472-2475.

429. Winnacker, M.; Rieger, B. Biobased Polyamides: Recent Advances in Basic and Applied Research. Macromol. Rapid Commun. 2016, 37 (17), 1391-1413.

430. Genas, M. Rilsan (Polyamid 11), Synthese Und Eigenschaften. Angew. Chemie 1962, 74 (15), $535-540$.

431. Baumann, H.; Bühler, M.; Fochem, H.; Hirsinger, F.; Zoebelein, H.; Falbe, J. Natural Fats and Oils-Renewable Raw Materials for the Chemical Industry. Angew. Chemie Int. Ed. English 1988, $27(1), 41-62$.

432. Arkema. Rilsan PA11: Created from a Renewable Source. Arkema Puteeaux, Fr. 2009.

433. Häger, H. VESTAMID Terra, Lifecycle Asessment of Biobased Polyamides. Evonik Degussa GmbH, Marl, Ger. 2011.

434. DSM. EcoPaXX : The Green Performer. DSM Eng. Plast. Galeen, Netherlands 2011.

435. Wang, M. S.; Huang, J. C. Nylon 1010 Properties and Applications. J. Polym. Eng. 1994, 13 (2), $155-174$.

436. Kuciel, S.; Kuniar, P.; Liber-Knec, A. Polyamides from Renewable Sources as Matrices of Short Fiber Reinforced Biocomposites. Polimery 2012, No. 9, 627.

437. Feldmann, M.; Bledzki, A. K. Bio-Based Polyamides Reinforced with Cellulosic Fibres Processing and Properties. Compos. Sci. Technol. 2014, 100, 113-120.

438. Samanta, S. R. Intrinsic Viscosity and Molecular Weight Measurement of Nylon 66 Polymers. $J$. Appl. Polym. Sci. 1992, 45 (9), 1635-1640.

439. Mutlu, H.; Meier, M. A. R. Unsaturated PA X,20 from Renewable Resources via Metathesis and Catalytic Amidation. Macromol. Chem. Phys. 2009, 210 (12), 1019-1025.

440. Greene, J. L.; Huffman, E. L.; Burks, R. E.; Sheehan, W. C.; Wolff, I. A. Nylon 1313: Synthesis and Polymerization of Monomers. J. Polym. Sci. Part A-1 Polym. Chem. 1967, 5 (2), 391-394.

441. Nieschlag, H. J.; Rothfus, J. A.; Sohns, V. E.; Perkins, R. B. Nylon-1313 from Brassylic Acid. Ind. Eng. Chem. Prod. Res. Dev. 1977, 16 (1), 101-107.

442. Wang, Y.; Liu, M.; Wang, Z.; Li, X.; Zhao, Q.; Fu, P.-F. Kinetics of Isothermal and Nonisothermal Crystallization of Nylon 1313. J. Appl. Polym. Sci. 2007, 104 (3), 1415-1422.

443. Samanta, S.; He, J.; Selvakumar, S.; Lattimer, J.; Ulven, C.; Sibi, M.; Bahr, J.; Chisholm, B. J. Polyamides Based on the Renewable Monomer, 1,13-Tridecane Diamine II: Synthesis and Characterization of Nylon 13,6. Polymer (Guildf). 2013, 54 (3), 1141-1149.

444. He, J.; Samanta, S.; Selvakumar, S.; Lattimer, J.; Ulven, C.; Sibi, M.; Bahr, J.; Chisholm, B. J. Polyamides Based on the Renewable Monomer, 1,13-Tridecane Diamine I: Synthesis and Characterization of Nylon 13,T. Green Mater. 2013, 1 (2), 114-124. 
445. Eschenfeldt, W. H.; Zhang, Y.; Samaha, H.; Eirich, L. D.; Wilson, C. R.; Mark, I.; Stols, L.; Donnelly, M. I. Transformation of Fatty Acids Catalyzed by Cytochrome P450 Monooxygenase Enzymes of Candida Tropicalis Transformation of Fatty Acids Catalyzed by Cytochrome P450 Monooxygenase Enzymes of Candida Tropicalis. Appl. Environ. Microbiol. 2003, 69 (10), 59925999.

446. Pardal, F.; Salhi, S.; Rousseau, B.; Tessier, M.; Claude, S.; Fradet, A. Unsaturated Polyamides from Bio-BasedZ-Octadec-9-Enedioic Acid. Macromol. Chem. Phys. 2008, 209 (1), 64-74.

447. Bennett, C.; Mathias, L. J. Synthesis and Characterization of Polyamides Containing Octadecanedioic Acid: Nylon-2,18, Nylon-3,18, Nylon-4,18, Nylon-6,18, Nylon-8,18, Nylon9,18, and Nylon-12,18. J. Polym. Sci. Part A Polym. Chem. 2005, 43 (5), 936-945.

448. Cui, X.; Li, W.; Yan, D.; Yuan, C.; Di Silvestro, G. Synthesis and Characterization of Polyamides X 18. J. Appl. Polym. Sci. 2005, 98 (4), 1565-1571.

449. Stempfle, F.; Quinzler, D.; Heckler, I.; Mecking, S. Long-Chain Linear C19 and C23 Monomers and Polycondensates from Unsaturated Fatty Acid Esters. Macromolecules 2011, 44 (11), 4159 4166.

450. Walther, G.; Deutsch, J.; Martin, A.; Baumann, F.-E.; Fridag, D.; Franke, R.; Köckritz, A. A, (Functionalized C19 Monomers. ChemSusChem 2011, 4 (8), 1052-1054.

451. Çavuş, S.; Gürkaynak, M. A. Influence of Monofunctional Reactants on the Physical Properties of Dimer Acid-Based Polyamides. Polym. Adv. Technol. 2006, 17 (1), 30-36.

452. HEIDARIAN, J.; GHASEM, N.; DAUD, W. Study on Kinetics of Polymerization of Dimer Fatty Acids with Ethylenediamine in the Presence of Catalyst. Chem. Eng. J. 2004, 100 (1-3), 85-93.

453. Chen, X.; Zhong, H.; Jia, L.; Ning, J.; Tang, R.; Qiao, J.; Zhang, Z. Polyamides Derived from Piperazine and Used for Hot-Melt Adhesives: Synthesis and Properties. Int. J. Adhes. Adhes. 2002, $22(1), 75-79$.

454. Unverferth, M.; Meier, M. A. R. Selective Formation of C36-Dimer Fatty Acids via Thiol-Ene Addition for Copolyamide Synthesis. Eur. J. Lipid Sci. Technol. 2016, 118 (10), 1470-1474.

455. Turunc, O.; Firdaus, M.; Klein, G.; Meier, M. A. R. Fatty Acid Derived Renewable Polyamides via Thiol-Ene Additions. Green Chem. 2012, 14 (9), 2577-2583.

456. Miao, X.; Malacea, R.; Fischmeister, C.; Bruneau, C.; Dixneuf, P. H. Ruthenium-Alkylidene Catalysed Cross-Metathesis of Fatty Acid Derivatives with Acrylonitrile and Methyl Acrylate: A Key Step toward Long-Chain Bifunctional and Amino Acid Compounds. Green Chem. 2011, 13 (10), 2911-2919.

457. Miao, X.; Fischmeister, C.; Dixneuf, P. H.; Bruneau, C.; Dubois, J. L.; Couturier, J. L. Polyamide Precursors from Renewable 10-Undecenenitrile and Methyl Acrylate via Olefin Cross-Metathesis. Green Chem. 2012, 14 (8), 2179-2183.

458. Winkler, M.; Steinbiß, M.; Meier, M. A. R. A More Sustainable Wohl-Ziegler Bromination: Versatile Derivatization of Unsaturated FAMEs and Synthesis of Renewable Polyamides. Eur. $J$. Lipid Sci. Technol. 2014, 116 (1), 44-51.

459. Winkler, M.; Meier, M. a. R. Highly Efficient Oxyfunctionalization of Unsaturated Fatty Acid Esters: An Attractive Route for the Synthesis of Polyamides from Renewable Resources. Green Chem. 2014, 16 (4), 1784-1788.

460. Oelmann, S.; Meier, M. A. R. Synthesis of Modified Polycaprolactams Obtained from Renewable Resources. Macromol. Chem. Phys. 2015, 216 (19), 1972-1981. 
461. Lligadas, G.; Ronda, J. C.; Galià, M.; Biermann, U.; Metzger, J. O. Synthesis and Characterization of Polyurethanes from Epoxidized Methyl Oleate Based Polyether Polyols as Renewable Resources. J. Polym. Sci. Part A Polym. Chem. 2006, 44 (1), 634-645.

462. Xu, Y.; Petrovic, Z.; Das, S.; Wilkes, G. L. Morphology and Properties of Thermoplastic Polyurethanes with Dangling Chains in Ricinoleate-Based Soft Segments. Polymer (Guildf). 2008, 49 (19), 4248-4258.

463. Petrović, Z. S.; Hong, D.; Javni, I.; Erina, N.; Zhang, F.; Ilavský, J. Phase Structure in Segmented Polyurethanes Having Fatty Acid-Based Soft Segments. Polymer (Guildf). 2013, 54 (1), 372-380.

464. Saralegi, A.; Rueda, L.; Fernández-d’Arlas, B.; Mondragon, I.; Eceiza, A.; Corcuera, M. A. Thermoplastic Polyurethanes from Renewable Resources: Effect of Soft Segment Chemical Structure and Molecular Weight on Morphology and Final Properties. Polym. Int. 2013, 62 (1), $106-115$.

465. Hojabri, L.; Jose, J.; Leao, A. L.; Bouzidi, L.; Narine, S. S. Synthesis and Physical Properties of Lipid-Based Poly(ester-Urethane)s, I: Effect of Varying Polyester Segment Length. Polymer (Guildf). 2012, 53 (17), 3762-3771.

466. Lluch, C.; Ronda, J. C.; Galià, M.; Lligadas, G.; Cádiz, V. Rapid Approach to Biobased Telechelics through Two One-Pot Thiol-Ene Click Reactions. Biomacromolecules 2010, 11 (6), 1646-1653.

467. Petrović, Z. S.; Xu, Y.; Milić, J.; Glenn, G.; Klamczynski, A. Biodegradation of Thermoplastic Polyurethanes from Vegetable Oils. J. Polym. Environ. 2010, 18 (2), 94-97.

468. Tang, D.; Noordover, B. A. J.; Sablong, R. J.; Koning, C. E. Metal-Free Synthesis of Novel Biobased Dihydroxyl-Terminated Aliphatic Polyesters as Building Blocks for Thermoplastic Polyurethanes. J. Polym. Sci. Part A Polym. Chem. 2011, 49 (13), 2959-2968.

469. Bueno-Ferrer, C.; Hablot, E.; Perrin-Sarazin, F.; Garrigós, M. C.; Jiménez, A.; Averous, L. Structure and Morphology of New Bio-Based Thermoplastic Polyurethanes Obtained From Dimeric Fatty Acids. Macromol. Mater. Eng. 2012, 297 (8), 777-784.

470. Bueno-Ferrer, C.; Hablot, E.; Garrigós, M. del C.; Bocchini, S.; Averous, L.; Jiménez, A. Relationship between Morphology, Properties and Degradation Parameters of Novative Biobased Thermoplastic Polyurethanes Obtained from Dimer Fatty Acids. Polym. Degrad. Stab. 2012, 97 (10), 1964-1969.

471. González-Paz, R. J.; Lluch, C.; Lligadas, G.; Ronda, J. C.; Galià, M.; Cádiz, V. A Green Approach toward Oleic- and Undecylenic Acid-Derived Polyurethanes. J. Polym. Sci. Part A Polym. Chem. 2011, 49 (11), 2407-2416.

472. Desroches, M.; Caillol, S.; Auvergne, R.; Boutevin, B. Synthesis of Pseudo-Telechelic Diols by Transesterification and Thiol-Ene Coupling. Eur. J. Lipid Sci. Technol. 2012, 114 (1), 84-91.

473. Palaskar, D. V; Boyer, A.; Cloutet, E.; Le Meins, J.-F.; Gadenne, B.; Alfos, C.; Farcet, C.; Cramail, H. Original Diols from Sunflower and Ricin Oils: Synthesis, Characterization, and Use as Polyurethane Building Blocks. J. Polym. Sci. Part A Polym. Chem. 2012, 50 (9), 1766-1782.

474. Maisonneuve, L.; Lebarbe, T.; Nguyen, T. H. N.; Cloutet, E.; Gadenne, B.; Alfos, C.; Cramail, H. Hydroxyl Telechelic Building Blocks from Fatty Acid Methyl Esters for the Synthesis of Poly(ester/amide Urethane)s with Versatile Properties. Polym. Chem. 2012, 3 (9), 2583-2595.

475. Boyer, A.; Lingome, C. E.; Condassamy, O.; Schappacher, M.; Moebs-Sanchez, S.; Queneau, Y.; Gadenne, B.; Alfos, C.; Cramail, H. Glycolipids as a Source of Polyols for the Design of Original Linear and Cross-Linked Polyurethanes. Polym. Chem. 2013, 4 (2), 296-306. 
476. Hojabri, L.; Kong, X.; Narine, S. S. Fatty Acid-Derived Diisocyanate and Biobased Polyurethane Produced from Vegetable Oil: Synthesis, Polymerization, and Characterization.

Biomacromolecules 2009, 10 (4), 884-891.

477. Hojabri, L.; Kong, X.; Narine, S. S. Novel Long Chain Unsaturated Diisocyanate from Fatty Acid: Synthesis, Characterization, and Application in Bio-Based Polyurethane. J. Polym. Sci. Part A Polym. Chem. 2010, 48 (15), 3302-3310.

478. More, A. S.; Lebarbé, T.; Maisonneuve, L.; Gadenne, B.; Alfos, C.; Cramail, H. Novel Fatty Acid Based Di-Isocyanates towards the Synthesis of Thermoplastic Polyurethanes. Eur. Polym. J. 2013, 49 (4), 823-833.

479. Duval, C.; Kébir, N.; Charvet, A.; Martin, A.; Burel, F. Synthesis and Properties of Renewable Nonisocyanate Polyurethanes (NIPUs) from Dimethylcarbonate. J. Polym. Sci. Part A Polym. Chem. 2015, 53 (11), 1351-1359.

480. Unverferth, M.; Kreye, O.; Prohammer, A.; Meier, M. A. R. Renewable Non-Isocyanate Based Thermoplastic Polyurethanes via Polycondensation of Dimethyl Carbamate Monomers with Diols. Macromol. Rapid Commun. 2013, 34 (19), 1569-1574.

481. Kreye, O.; Wald, S.; Meier, M. A. R. Introducing Catalytic Lossen Rearrangements: Sustainable Access to Carbamates and Amines. Adv. Synth. Catal. 2013, 355 (1), 81-86.

482. More, A. S.; Gadenne, B.; Alfos, C.; Cramail, H. AB Type Polyaddition Route to Thermoplastic Polyurethanes from Fatty Acid Derivatives. Polym. Chem. 2012, 3 (6), 1594-1605.

483. Palaskar, D. V; Boyer, A.; Cloutet, E.; Alfos, C.; Cramail, H. Synthesis of Biobased Polyurethane from Oleic and Ricinoleic Acids as the Renewable Resources via the AB-Type Self-Condensation Approach. Biomacromolecules 2010, 11 (5), 1202-1211.

484. Boyer, A.; Cloutet, E.; Tassaing, T.; Gadenne, B.; Alfos, C.; Cramail, H. Solubility in CO2 and Carbonation Studies of Epoxidized Fatty Acid Diesters: Towards Novel Precursors for Polyurethane Synthesis. Green Chem. 2010, 12 (12), 2205-2213.

485. Foltran, S.; Maisonneuve, L.; Cloutet, E.; Gadenne, B.; Alfos, C.; Tassaing, T.; Cramail, H. Solubility in $\mathrm{CO} 2$ and Swelling Studies by in Situ IR Spectroscopy of Vegetable-Based Epoxidized Oils as Polyurethane Precursors. Polym. Chem. 2012, 3 (2), 525-532.

486. Maisonneuve, L.; More, A. S.; Foltran, S.; Alfos, C.; Robert, F.; Landais, Y.; Tassaing, T.; Grau, E.; Cramail, H. Novel Green Fatty Acid-Based Bis-Cyclic Carbonates for the Synthesis of Isocyanate-Free Poly(hydroxyurethane Amide)s. RSC Adv. 2014, 4 (49), 25795-25803.

487. Tomita, H.; Sanda, F.; Endo, T. Reactivity Comparison of Five- and Six-Membered Cyclic Carbonates with Amines: Basic Evaluation for Synthesis of Poly(hydroxyurethane). J. Polym. Sci. Part A Polym. Chem. 2000, 39 (1), 162-168.

488. Tomita, H.; Sanda, F.; Endo, T. Polyaddition Behavior of Bis(five- and Six-Membered Cyclic Carbonate)s with Diamine. J. Polym. Sci. Part A Polym. Chem. 2001, 39 (6), 860-867.

489. Tomita, H.; Sanda, F.; Endo, T. Polyaddition of Bis(seven-Membered Cyclic Carbonate) with Diamines: A Novel and Efficient Synthetic Method for Polyhydroxyurethanes. J. Polym. Sci. Part A Polym. Chem. 2001, 39 (23), 4091-4100.

490. Besse, V.; Foyer, G.; Auvergne, R.; Caillol, S.; Boutevin, B. Access to Nonisocyanate Poly(thio)urethanes: A Comparative Study. J. Polym. Sci. Part A Polym. Chem. 2013, 51 (15), 3284-3296.

491. Kihara, N.; Kushida, Y.; Endo, T. Optically Active Poly(hydroxyurethane)s Derived from Cyclic 
Carbonate and L-Lysine Derivatives. J. Polym. Sci. Part A Polym. Chem. 1996, 34 (11), $2173-$ 2179.

492. Steblyanko, A.; Choi, W.; Sanda, F.; Endo, T. Addition of Five-Membered Cyclic Carbonate with Amine and Its Application to Polymer Synthesis. J. Polym. Sci. Part A Polym. Chem. 2000, 38 (13), 2375-2380.

493. Kihara, N.; Endo, T. Synthesis and Properties of Poly(hydroxyurethane)s. J. Polym. Sci. Part A Polym. Chem. 1993, 31 (11), 2765-2773.

494. Fleischer, M.; BlatTmann, H.; Mulhaupt, R. Glycerol-, Pentaerythritol- and TrimethylolpropaneBased Polyurethanes and Their Cellulose Carbonate Composites Prepared via the Non-Isocyanate Route with Catalytic Carbon Dioxide Fixation. Green Chem. 2013, 15 (4), 934-942.

495. Keul, H.; Mommer, S.; Möller, M. Poly(amide Urethane)s with Functional/reactive Side Groups Based on a Bis-Cyclic Bio-Based Monomer/coupling Agent. Eur. Polym. J. 2013, 49 (4), 853864.

496. van Velthoven, J. L. J.; Gootjes, L.; van Es, D. S.; Noordover, B. A. J.; Meuldijk, J. Poly(hydroxy Urethane)s Based on Renewable Diglycerol Dicarbonate. Eur. Polym. J. 2015, 70, 125-135.

497. Lamarzelle, O.; Durand, P.-L.; Wirotius, A.-L.; Chollet, G.; Grau, E.; Cramail, H. Activated Lipidic Cyclic Carbonates for Non-Isocyanate Polyurethane Synthesis. Polym. Chem. 2016, 7, $1439-1451$.

498. Lamarzelle, O.; Hibert, G.; Lecommandoux, S.; Grau, E.; Cramail, H. A Thioglycerol Route to Bio-Based Bis-Cyclic Carbonates: Poly(hydroxyurethane) Preparation and Post-Functionalization. Polym. Chem. 2017, 8 (22), 3438-3447.

499. Maisonneuve, L.; Wirotius, A.-L.; Alfos, C.; Grau, E.; Cramail, H. Fatty Acid-Based (Bis) 6Membered Cyclic Carbonates as Efficient Isocyanate Free Poly(hydroxyurethane) Precursors. Polym. Chem. 2014, 5, 6142-6147.

500. Desroches, M.; Benyahya, S.; Besse, V.; Auvergne, R.; Boutevin, B.; Caillol, S. Synthesis of BioBased Building Blocks from Vegetable Oils: A Platform Chemicals Approach. Lipid Technol. 2014, 26 (2), 35-38.

501. Edlund, U.; Albertsson, A.-C. Polyesters Based on Diacid Monomers. Adv. Drug Deliv. Rev. 2003, 55 (4), 585-609.

502. Zhang, J.; Li, J.; Tang, Y.; Lin, L.; Long, M. Advances in Catalytic Production of Bio-Based Polyester Monomer 2,5-Furandicarboxylic Acid Derived from Lignocellulosic Biomass. Carbohydr. Polym. 2015, 130, 420-428.

503. Bakare, I. O.; Pavithran, C.; Okieimen, F. E.; Pillai, C. K. S. Polyesters from Renewable Resources: Preparation and Characterization. J. Appl. Polym. Sci. 2006, 100 (5), 3748-3755.

504. Fakirov, S.; Wiley InterScience (Online service). Transreactions in Condensation Polymers; Wiley-VCH, 1999.

505. Zia, K. M.; Noreen, A.; Zuber, M.; Tabasum, S.; Mujahid, M. Recent Developments and Future Prospects on Bio-Based Polyesters Derived from Renewable Resources: A Review. Int. J. Biol. Macromol. 2016, 82, 1028-1040.

506. Quinzler, D.; Mecking, S. Linear Semicrystalline Polyesters from Fatty Acids by Complete Feedstock Molecule Utilization. Angew. Chemie Int. Ed. 2010, 49 (25), 4306-4308.

507. Ravi Kumar, M. N. V. Handbook of Polyester Drug Delivery Systems. 
508. Kunduru, K. R.; Basu, A.; Haim Zada, M.; Domb, A. J. Castor Oil-Based Biodegradable Polyesters. Biomacromolecules 2015, 16 (9), 2572-2587.

509. Ebata, H.; Toshima, K.; Matsumura, S. Lipase-Catalyzed Synthesis and Curing of HighMolecular-Weight Polyricinoleate. Macromol. Biosci. 2007, 7 (6), 798-803.

510. Totaro, G.; Cruciani, L.; Vannini, M.; Mazzola, G.; Di Gioia, D.; Celli, A.; Sisti, L. Synthesis of Castor Oil-Derived Polyesters with Antimicrobial Activity. Eur. Polym. J. 2014, 56, 174-184.

511. Kelly, A. R.; Hayes, D. G. Lipase-Catalyzed Synthesis of Polyhydric Alcohol-Poly(ricinoleic Acid) Ester Star Polymers. J. Appl. Polym. Sci. 2006, 101 (3), 1646-1656.

512. Ebata, H.; Yasuda, M.; Toshima, K.; Matsumura, S. Poly (Ricinoleic Acid) Based Novel Thermosetting Elastomer. J. Oleo Sci. 2008, 57 (6), 315-320.

513. Mutlu, H.; Meier, M. A. R. Castor Oil as a Renewable Resource for the Chemical Industry. Eur. J. Lipid Sci. Technol. 2010, 112 (1), 10-30.

514. Hiremath, J. G.; Kusum Devi, V.; Devi, K.; Domb, A. J. Biodegradable Poly(sebacic Acid-CoRicinoleic-Ester Anhydride) Tamoxifen Citrate Implants: Preparation Andin Vitro Characterization. J. Appl. Polym. Sci. 2008, 107 (5), 2745-2754.

515. Shikanov, A.; Vaisman, B.; Shikanov, S.; Domb, A. J. Efficacy of Poly(sebacic Acid-CoRicinoleic Acid) Biodegradable Delivery System for Intratumoral Delivery of Paclitaxel. $J$. Biomed. Mater. Res. Part A 2009, 9999 A (4), NA-NA.

516. Petrović, Z. S.; Milić, J.; Xu, Y.; Cvetković, I. A Chemical Route to High Molecular Weight Vegetable Oil-Based Polyhydroxyalkanoate. Macromolecules 2010, 43 (9), 4120-4125.

517. Testud, B.; Pintori, D.; Grau, E.; Taton, D.; Cramail, H. Hyperbranched Polyesters by Polycondensation of Fatty Acid-Based AB $n$-Type Monomers. Green Chem. 2017, 6-11.

518. Yang, Y.; Lu, W.; Zhang, X.; Xie, W.; Cai, M.; Gross, R. A. Two-Step Biocatalytic Route to Biobased Functional Polyesters from $\omega$-Carboxy Fatty Acids and Diols. Biomacromolecules 2010, 11 (1), 259-268.

519. Türünç, O.; Meier, M. A. R. Fatty Acid Derived Monomers and Related Polymers via Thiol-Ene (Click) Additions. Macromol. Rapid Commun. 2010, 31 (20), 1822-1826.

520. Lebarbé, T.; Maisonneuve, L.; Nga Nguyen, T. H.; Gadenne, B.; Alfos, C.; Cramail, H.; Gross, R. A.; Cramail, H. Methyl 10-Undecenoate as a Raw Material for the Synthesis of Renewable SemiCrystalline Polyesters and Poly(ester-Amide)s. Polym. Chem. 2012, 3 (10), 2842.

521. Trzaskowski, J.; Quinzler, D.; Bährle, C.; Mecking, S. Aliphatic Long-Chain C20 Polyesters from Olefin Metathesis. Macromol. Rapid Commun. 2011, 32 (17), 1352-1356.

522. Vilela, C.; Silvestre, A. J. D.; Meier, M. A. R. Plant Oil-Based Long-Chain C26 Monomers and Their Polymers. Macromol. Chem. Phys. 2012, 213 (21), 2220-2227.

523. Warwel, S.; Tillack, J.; Demes, C.; Kunz, M. Polyesters of $\omega$-Unsaturated Fatty Acid Derivatives. Macromol. Chem. Phys. 2001, 202 (7), 1114-1121.

524. Bauch, C. G.; Wagener, K. B.; Boncella, J. M. Acyclic Diene Metathesis (ADMET) Polymerization. Synthesis of an Unsaturated Polyester. Die Makromol. Chemie, Rapid Commun. 1991, 12 (7), 413-417.

525. Hall, A. J.; Hodge, P.; Kamau, S. D.; Ben-Haida, A. Acyclic Diene Metathesis (ADMET) Polymerization of Allyl Undec-10-Enoate and Some Related Esters. J. Organomet. Chem. 2006, 
$691(24-25), 5431-5437$.

526. Stempfle, F.; OrTmann, P.; Mecking, S. Which Polyesters Can Mimic Polyethylene? Macromol. Rapid Commun. 2013, 34 (1), 47-50.

527. Türünç, O.; Meier, M. a. R. Thiol-Ene vs. ADMET: A Complementary Approach to Fatty AcidBased Biodegradable Polymers. Green Chem. 2011, 13 (2), 314.

528. Fokou, P. A.; Meier, M. A. R. Use of a Renewable and Degradable Monomer to Study the Temperature-Dependent Olefin Isomerization during ADMET Polymerizations. J. Am. Chem. Soc. 2009, 131 (5), 1664-1665.

529. Fokou, P. A.; Meier, M. A. R. Studying and Suppressing Olefin Isomerization Side Reactions During ADMET Polymerizations. Macromol. Rapid Commun. 2010, 31 (4), 368-373.

530. Nivinskiene, O.; Butkiene, R.; Mockute, D. Changes in the Chemical Composition of Essential Oil of Angelica Archangelica L . Roots during Storage. Chemija 2003, 14 (1), 52-56.

531. Pascual, A.; Sardon, H.; Veloso, A.; Ruipérez, F.; Mecerreyes, D. Organocatalyzed Synthesis of Aliphatic Polyesters from Ethylene Brassylate: A Cheap and Renewable Macrolactone. ACS Macro Lett. 2014, 3 (9), 849-853.

532. Raia Slivniak; Abraham J. Domb*. Macrolactones and Polyesters from Ricinoleic Acid. 2005.

533. Gargouri, M.; Drouet, P.; Legoy, M.-D. Synthesis of a Novel Macrolactone by Lipase-Catalyzed Intra-Esterification of Hydroxy-Fatty Acid in Organic Media. J. Biotechnol. 2002, 92 (3), 259266.

534. de Geus, M.; van der Meulen, I.; Goderis, B.; van Hecke, K.; Dorschu, M.; van der Werff, H.; Koning, C. E.; Heise, A. Performance Polymers from Renewable Monomers: High Molecular Weight Poly(pentadecalactone) for Fiber Applications. Polym. Chem. 2010, 1 (4), 525.

535. Kirpal S. Bisht; Lori A. Henderson, and; Gross*, R. A.; Kaplan, D. L.; Swift, G. EnzymeCatalyzed Ring-Opening Polymerization of $\omega$-Pentadecalactone $\dagger$. 1997.

536. Cai, J.; Liu, C.; Cai, M.; Zhu, J.; Zuo, F.; Hsiao, B. S.; Gross, R. A. Effects of Molecular Weight on Poly( $\omega$-Pentadecalactone) Mechanical and Thermal Properties. Polymer (Guildf). 2010, 51 (5), 1088-1099.

537. Nakayama, Y.; Watanabe, N.; Kusaba, K.; Sasaki, K.; Cai, Z.; Shiono, T.; Tsutsumi, C. High Activity of Rare Earth Tetrahydroborates for Ring-Opening Polymerization of $\omega$ Pentadecalactone. J. Appl. Polym. Sci. 2011, 121 (4), 2098-2103.

538. Zhong, Z.; Dijkstra, P. J.; Feijen, J. Controlled Ring-Opening Polymerization of -Pentadecalactone with Yttrium Isopropoxide as an Initiator. Macromol. Chem. Phys. 2000, 201 (12), 1329-1333.

539. Bouyahyi, M.; Pepels, M. P. F.; Heise, A.; Duchateau, R. $\omega$-Pentandecalactone Polymerization and $\omega$-Pentadecalactone/ $\varepsilon$-Caprolactone Copolymerization Reactions Using Organic Catalysts. Macromolecules 2012, 45 (8), 3356-3366.

540. van der Meulen, I.; de Geus, M.; Antheunis, H.; Deumens, R.; Joosten, E. A. J.; Koning, C. E.; Heise, A. Polymers from Functional Macrolactones as Potential Biomaterials: Enzymatic Ring Opening Polymerization, Biodegradation, and Biocompatibility. Biomacromolecules 2008, 9 (12), 3404-3410.

541. Mutlu, H.; Ruiz, J.; Solleder, S. C.; Meier, M. A. R.; Meier, M. A. R.; Cádiz, V.; Xiao, F.; Wei, W.; Sun, Y. TBD Catalysis with Dimethyl Carbonate: A Fruitful and Sustainable Alliance. Green Chem. 2012, 14 (6), 1728. 
542. More, A. S.; Palaskar, D. V.; Cloutet, E.; Gadenne, B.; Alfos, C.; Cramail, H.; Wang, F. Aliphatic Polycarbonates and Poly(ester Carbonate)s from Fatty Acid Derived Monomers. Polym. Chem. 2011, 2 (12), 2796.

543. Vanderhenst, R.; Miller, S. A. Polycarbonates from Biorenewable Diols via Carbonate Metathesis Polymerization. Green Mater. 2013, 1 (2), 64-78.

544. Zhang, Y.-Y.; Zhang, X.-H.; Wei, R.-J.; Du, B.-Y.; Fan, Z.-Q.; Qi, G.-R.; Qi, G. R.; Qi, G. R. Synthesis of Fully Alternating Polycarbonate with Low Tg from Carbon Dioxide and Bio-Based Fatty Acid. RSC Adv. 2014, 4 (68), 36183.

545. Winnacker, M.; Rieger, B. Recent Progress in Sustainable Polymers Obtained from Cyclic Terpenes: Synthesis, Properties, and Application Potential. ChemSusChem 2015, 8 (15), 24552471.

546. BreiTmaier, E. Terpenes: Flavors, Fragrances, Pharmaca, Pheromones; Wiley-VCH, Ed.; 2006.

547. Sun, I.; Wang, H.; Kashiwada, Y.; Shen, J.; Cosentino, L. M.; Chen, C.; Yang, L.; Lee, K. AntiAIDS Agents . 34 . $\uparrow$ Synthesis and Structure - Activity Relationships of Betulin Derivatives as Anti-HIV Agents. J. Med. Chem. 1998, 41, 4648-4657.

548. Cichewicz, R. H.; Kouzi, S. A. Chemistry, Biological Activity, and Chemotherapeutic Potential of Betulinic Acid for the Prevention and TreaTment of Cancer and HIV Infection. Med. Res. Rev. 2004, 24 (1), 90-114.

549. Gandini, A. The Irruption of Polymers from Renewable Resources on the Scene of Macromolecular Science and Technology. Green Chem. 2011, 13 (5), 1061.

550. Corma Canos, A.; Iborra, S.; Velty, A. Chemical Routes for the Transformation of Biomass into Chemicals. Chem. Rev. 2007, 107 (6), 2411-2502.

551. Belgacem, M.; Gandini, A. Monomers, Polymers and Composites from Renewable Resources; 2008.

552. Fomine, S.; Tlenkopatchev, M. A. Metathesis Transformations of Terpenes. Computational Modeling of (-)-??-Pinene Ring Opening by Ruthenium and Tungsten Carbene Catalysts. $J$. Organomet. Chem. 2012, 701, 68-74.

553. Strick, B. F.; Delferro, M.; Geiger, F. M.; Thomson, R. J. Investigations into Apopinene as a Biorenewable Monomer for Ring-Opening Metathesis Polymerization. ACS Sustain. Chem. Eng. 2015, 3 (7), 1278-1281.

554. Miyaji, H.; Satoh, K.; Kamigaito, M. Bio-Based Polyketones by Selective Ring-Opening Radical Polymerization of ??-Pinene-Derived Pinocarvone. Angew. Chemie - Int. Ed. 2016, 55 (4), 13721376.

555. Sainz, M. F.; Souto, J. A.; Regentova, D.; Johansson, M. K. G.; Timhagen, S. T.; Irvine, D. J.; Buijsen, P.; Koning, C. E.; Stockman, R. A.; Howdle, S. M. A Facile and Green Route to Terpene Derived Acrylate and Methacrylate Monomers and Simple Free Radical Polymerisation to Yield New Renewable Polymers and Coatings. Polym. Chem. 2016, 7 (16), 2882-2887.

556. Wu, G.; Kong, Z.; Huang, H.; Chen, J.; Chu, F. Synthesis, Characterization, and Properties of Polyols from Hydrogenated Terpinene - Maleic Ester Type Epoxy Resin. J. Appl. Polym. Sci. 2009, 113, 2894-2901.

557. Kukhta, N. A.; Vasilenko, I. V; Kostjuk, S. V. Green Chemistry Catalyst : Toward Sustainable Plastics from Renewable Biomass Resources $\uparrow$. 2011, 2362-2364. 
558. Wang, Y.; Li, A.-L.; Liang, H.; Lu, J. Reversible Addition-fragmentation Chain Transfer Radical Copolymerization of $\beta$-Pinene and Methyl Acrylate. Eur. Polym. J. 2006, 42 (10), 2695-2702.

559. Firdaus, M.; Montero De Espinosa, L.; Meier, M. A. R. Terpene-Based Renewable Monomers and Polymers via Thiol-Ene Additions. Macromolecules 2011, 44 (18), 7253-7262.

560. Firdaus, M.; Meier, M. A. R. Renewable Polyamides and Polyurethanes Derived from Limonene. Green Chem. 2013, 15 (2), 370.

561. Byrne, C. M.; Allen, S. D.; Lobkovsky, E. B.; Coates, G. W. Alternating Copolymerization of Limonene Oxide and Carbon Dioxide. J. Am. Chem. Soc. 2004, 126 (37), 11404-11405.

562. Hauenstein, O.; Reiter, M.; Agarwal, S.; Rieger, B.; Greiner, A. Bio-Based Polycarbonate from Limonene Oxide and CO 2 with High Molecular Weight, Excellent Thermal Resistance, Hardness and Transparency. Green Chem. 2016, 18 (3), 760-770.

563. Hauenstein, O.; Agarwal, S.; Greiner, A. Bio-Based Polycarbonate as Synthetic Toolbox. Nat. Commun. 2016, 7 (May), 1-7.

564. Bähr, M.; Bitto, A.; Mülhaupt, R. Cyclic Limonene Dicarbonate as a New Monomer for NonIsocyanate Oligo- and Polyurethanes (NIPU) Based upon Terpenes. Green Chem. 2012, 14 (5), 1447.

565. Kobayashi, S.; Lu, C.; Hoye, T. R.; Hillmyer, M. A. Controlled Polymerization of a Cyclic Diene Prepared from the Ring-Closing Metathesis of a Naturally Occurring Monoterpene. J. Am. Chem. Soc. 2009, 131 (23), 7960-7961.

566. Busch, H.; Stempfle, F.; Heß, S.; Grau, E.; Mecking, S. Selective Isomerization-carbonylation of a Terpene Trisubstituted Double Bond. Green Chem. 2014, 16 (10), 4541-4545.

567. Lowe, J. R.; Martello, M. T.; Tolman, W. B.; Hillmyer, M. A. Functional Biorenewable Polyesters from Carvone-Derived Lactones. Polym. Chem. 2011, 2 (3), 702-708.

568. Lowe, J. R.; Tolman, W. B.; Hillmyer, M. A. Oxidized Dihydrocarvone as a Renewable Multifunctional Monomer for the Synthesis of Shape Memory Polyesters. Biomacromolecules 2009, 10, 2003.

569. Zhang, D.; Hillmyer, M. A.; Tolman, W. B. Catalytic Polymerization of a Cyclic Ester Derived from A "cool" natural Precursor. Biomacromolecules 2005, 6 (4), 2091-2095.

570. Wanamaker, C. L.; Bluemle, M. J.; Pitet, L. M.; O’Leary, L. E.; Tolman, W. B.; Hillmyer, M. A. Consequences of Polylactide Stereochemistry on the Properties of Polylactide-PolymenthidePolylactide Thermoplastic Elastomers. Biomacromolecules 2009, 10 (10), 2904-2911.

571. Wanamaker, C. L.; O’Leary, L. E.; Lynd, N. A.; Hillmeyer, M. A.; Tolman, W. B. RenewableResource Thermoplastic Elastomers Based on Polylactide and Polymenthide. Biomacromolecules 2007, 8 (11), 3634-3640.

572. Shin, J.; Martello, M. T.; Shrestha, M.; Wissinger, J. E.; Tolman, W. B.; Hillmyer, M. A. PressureSensitive Adhesives from Renewable Triblock Copolymers. Macromolecules 2011, 44 (1), 87-94.

573. Atta, A. M.; Mansour, R.; Abdou, M. I.; Sayed, A. M. Epoxy Resins from Rosin Acids: Synthesis and Characterization. Polym. Adv. Technol. 2004, 15 (9), 514-522.

574. Atta, A. M.; Elsaeed, A. M.; Farag, R. K.; El-Saeed, S. M. Synthesis of Unsaturated Polyester Resins Based on Rosin Acrylic Acid Adduct for Coating Applications. React. Funct. Polym. 2007, 67 (6), 549-563. 
575. Bicu, I.; Mustata, F. Water Soluble Polymers from Diels-Alder Adducts of Abietic Acid as Paper Additives. Macromol. Mater. Eng. 2000, 280-281 (1), 47-53.

576. Bicu, I.; Mustata, F. Polymers from a Levopimaric Acid-Acrylonitrile Diels-Alder Adduct: Synthesis and Characterization. J. Polym. Sci. Part A Polym. Chem. 2005, 43 (24), 6308-6322.

577. Bicu, I.; Mustata, F. Polymers from a Levopimaric Acid-Acrylic Acid Diels-Alder Adduct: Synthesis and Characterization. J. Polym. Sci. Part A Polym. Chem. 2007, 45 (24), 5979-5990.

578. Bicu, I.; Mustata, F. Polyhydroxyimides from Resinic Acids. Polimery 2000, 45 (4), 258.

579. Mustata, F.; Bicu, I. A Novel Route for Synthesizing Esters and Polyesters from the Diels-Alder Adduct of Levopimaric Acid and Acrylic Acid. Eur. Polym. J. 2010, 46 (6), 1316-1327.

580. Kim, S. J.; Kim, B. J.; Jang, D. W.; Kim, S. H.; Park, S. Y.; Lee, J. H.; Lee, S. D.; Choi, D. H. Photoactive Polyamideimides Synthesized by the Polycondensation of Azo-Dye Diamines and Rosin Derivative. J. Appl. Polym. Sci. 2001, 79 (4), 687-695.

581. Atta, A. M.; El-Saeed, S. M.; Farag, R. K. New Vinyl Ester Resins Based on Rosin for Coating Applications. React. Funct. Polym. 2006, 66 (12), 1596-1608.

582. Lee, J. S.; Hong, S. Il. Synthesis of Acrylic Rosin Derivatives and Application as Negative Photoresist. Eur. Polym. J. 2002, 38 (2), 387-392.

583. Kim, H.-J. Synthesis and Characteristics of Photoactive-Hydrogenated Rosin Epoxy Methacrylate for Pressure Sensitive Adhesives. J. Appl. Polym. Sci. 2009, 111, 1172-1176.

584. Chu, F. Study on the Synthesis, Characterization, and Kinetic of Bulk Polymerization of Disproportionated Rosin (B-Acryloxyl Ethyl) Ester. J. Appl. Polym. Sci. 2009, 113, 3757-3765.

585. Wilbon, P. A.; Zheng, Y.; Yao, K.; Tang, C. Renewable Rosin Acid-Degradable Caprolactone Block Copolymers by Atom Transfer Radical Polymerization and Ring-Opening Polymerization. Macromolecules 2010, 43 (21), 8747-8754.

586. Zheng, Y.; Yao, K.; Lee, J.; Chandler, D.; Wang, J.; Wang, C.; Chu, F.; Tang, C. Well-Defined Renewable Polymers Derived from Gum Rosin. Macromolecules 2010, 43 (14), 5922-5924.

587. Wang, J.; Yao, K.; Korich, A. L.; Li, S.; Ma, S.; Ploehn, H. J.; Iovine, P. M.; Wang, C.; Chu, F.; Tang, C. Combining Renewable Gum Rosin and Lignin: Towards Hydrophobic Polymer Composites by Controlled Polymerization. J. Polym. Sci. Part A Polym. Chem. 2011, 49 (17), $3728-3738$.

588. Chen, Y.; Wilbon, P. A.; Chen, Y. P.; Zhou, J.; Nagarkatti, M.; Wang, C.; Chu, F.; Decho, A. W.; Tang, C. Amphipathic Antibacterial Agents Using Cationic Methacrylic Polymers with Natural Rosin as Pendant Group. RSC Adv. 2012, 2 (27), 10275.

589. Yao, K.; Wang, J.; Zhang, W.; Lee, J. S.; Wang, C.; Chu, F.; He, X.; Tang, C. Degradable RosinEster_Caprolactone Graft Copolymers. Biomacromolecules 2011, 12, 2171-2177.

590. Wang, J.; Chen, Y. P.; Yao, K.; Wilbon, P. a.; Zhang, W.; Ren, L.; Zhou, J.; Nagarkatti, M.; Wang, C.; Chu, F.; et al. Robust Antimicrobial Compounds and Polymers Derived from Natural Resin Acids. Chem. Commun. 2012, 48 (6), 916.

591. Mantzaridis, C.; Brocas, A.-L.; Llevot, A.; Cendejas, G.; Auvergne, R.; Caillol, S.; Carlotti, S.; Cramail, H. Rosin Acid Oligomers as Precursors of DGEBA-Free Epoxy Resins. Green Chem. 2013, 15, 3091-3098.

592. Liu, X.; Xin, W.; Zhang, J. Rosin-Based Acid Anhydrides as Alternatives to Petrochemical Curing 
Agents. Green Chem. 2009, 11 (7), 1018.

593. Wang, H.; Liu, X.; Liu, B.; Zhang, J.; Xian, M. Synthesis of Rosin-Based Flexible AnhydrideType Curing Agents and Properties of the Cured Epoxy. Polym. Int. 2009, 58 (12), 1435-1441.

594. Wang, H.; Liu, B.; Liu, X.; Zhang, J.; Xian, M. Synthesis of Biobased Epoxy and Curing Agents Using Rosin and the Study of Cure Reactions. Green Chem. 2008, 10 (11), 1190.

595. Liu, X.; Xin, W.; Zhang, J. Rosin-Derived Imide-Diacids as Epoxy Curing Agents for Enhanced Performance. Bioresour. Technol. 2010, 101 (7), 2520-2524. 